\title{
Integrating behavior, hormones and genes associated with the primate HPA-axis
}

\author{
Dissertation \\ zur Erlangung des mathematisch-naturwissenschaftlichen Doktorgrades \\ "Doctor rerum naturalium" \\ der Georg-August-Universität Göttingen \\ im Promotionsprogramm Behavior and Cognition (BeCog) \\ der Georg-August University School of Science (GAUSS) \\ vorgelegt von \\ Daria Raffaella Gutleb \\ aus Klagenfurt
}

Göttingen, 2018 


\section{$\underline{\text { Betreuungsausschuss }}$}

Prof. Dr. Julia Ostner, Verhaltensökologie, Georg-August Universität Göttingen

PD Dr. Oliver Schülke, Verhaltensökologie, Georg-August Universität Göttingen

PD Dr. Christian Roos, Primatengenetik, Deutsches Primatenzentrum

\section{Mitglieder der Prüfungskommission}

Referentin: Prof. Dr. Julia Ostner, Verhaltensökologie, Georg-August Universität Göttingen

Koreferent: PD Dr. Christian Roos, Primatengenetik, Deutsches Primatenzentrum

\section{Weitere Mitglieder der Prüfungskommission}

PD Dr. Oliver Schülke, Verhaltensökologie, Georg-August Universität Göttingen

Prof. Dr. Lars Penke, Biologische Persönlichkeitspsychologie, Georg-August Universität Göttingen

Dr. Claudia Fichtel, Verhaltensökologie \& Soziobiologie, Deutsches Primatenzentrum

Prof. Dr. Mark Maraun, Tierökologie, Georg-August Universität Göttingen 
für $A b u$ 



\section{Table of contents}

Acknowledgements

Summary

Zusammenfassung 7

Chapter $1 \quad$ General introduction $\ldots \ldots \ldots$

1.1 Preface

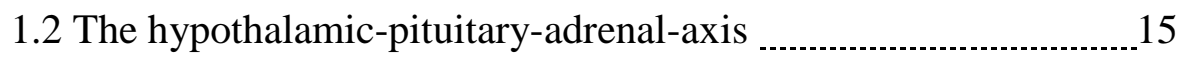

1.2.1 An evolutionary conserved pathway _.......................... 15

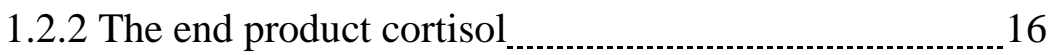

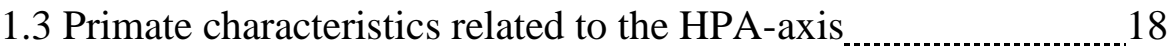

1.3.1 Aggression

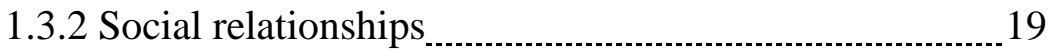

1.4 Sources of inter-individual differences: introducing the era of behavioral genetics

1.4.1 Candidate genes and the single-locus approach: a focus on COMT

1.4.2 The multi-locus approach: a focus on genetic risk scores 28

1.5 Study species: the Assamese macaque (Macaca assamensis)_..... 31

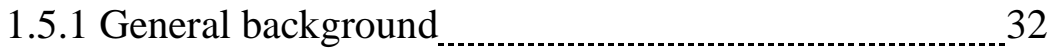

1.5.2 Hierarchy, sociality and seasonality _............................ 33

1.5.3 Assamese macaques in Phu Khieo Wildlife Sanctuary.35

1.6 Aims and approaches: studying genetic influences on phenotypes associated with the HPA-axis in a natural primate population.

Chapter 2 Study 1: Non-invasive genotyping with a massively parallel sequencing panel for the detection of SNPs in HPA-axis genes 
Chapter 3 Study 2: COMT Val ${ }^{158}$ Met moderates the link between rank and aggression in a non-human primate

Chapter 4 Study 3: A multi-locus genetic risk score modulates social buffering of HPA axis activity in wild male primates 79

Chapter 5 General discussion 97

5.1 Summary of the results 98

5.2 Limitations of the studies and the need for technological advances 100

5.2.1 Limitations 100

5.2.2 A brief comment on the need for technological advances

5.3 Effects of genotype on hormones and behavior in Assamese macaques

5.3.1 How genetic variation shapes inter-individual differences

5.3.2 An evolutionary scenario

5.4 Behavioral genetics of the HPA-axis 116

5.4.1 The heterogeneity of defining and measuring HPA-axis activity and social behavior

5.4.2 The effects of social bonds

5.5 Mammalian model organisms for the study of behavioral genetics

5.5.1 From mice to men

5.5.2 The genus Macaca in the lab and the wild 125 5.6 Conclusions and future directions

Supplementary material 135

References 162

Curriculum vitae 


\section{Acknowledgements}

First of all, I would like to thank Julia Ostner and Oliver Schülke for offering me the $\mathrm{PhD}$ position of my dreams. I am grateful for the opportunity to work with wild monkeys in your long-term field project and to extend my skills in the laboratory. Thank you for your trust and openness to incorporate my ideas into the $\mathrm{PhD}$ project. I really appreciate that you are so accessible and permanently available for questions. Also, thank you for letting me work in peace and quiet in a luxurious office on "the other side".

Many thanks go to Christian Roos for being a member of my thesis committee and my supervisor during one intense year in the genetics lab. Thank you for your help with troubleshooting, making assays work, your accessibility and answering e-mails at the speed of light. I especially appreciate your friendliness and optimistic attitude. Thank you for your motivating words and saying that working with me is like "Brezn backn". I guess you will never forget that you should have a "Mistkübel" in your office.

I thank Lars Penke, Claudia Fichtel and Mark Maraun for agreeing to be part of the committee in my oral defense. I am grateful to my collaborators at the DPZ. Special thanks go to Michael Heistermann for analyzing the precious urine samples that I collected and exported from Thailand. I thank Angela Noll for the support with the bioinformatics data, the nice time spent together in front of a computer analyzing huge amounts of data and for always having an open ear. Also, I thank Andrea Heistermann, Christiane Schwarz, Franziska Aron and Nico Westphal for their help in the laboratory. I thank the members of the Leibniz-ScienceCampus for valuable discussions. My special thanks go to Holger Sennhenn-Reulen for help with the statistics and Christian Schlögl for organizational advice. Further, I would like to thank the members of BeCog and the RTG2070 for the fruitful time spent together, and especially Rebecca Jürgens who has been extremely helpful regarding all organizational issues during my $\mathrm{PhD}$.

I would like to thank the National Research Council of Thailand and the Department of National Parks, Wildlife and Plant Conservation for permission to conduct this study. I thank J. Prabnasuk, K. Nitaya T. Wongsnak, M. Pongjantarasatien and K. Kreetiyutanont, M. Kumsuk, W. Saenphala from Phu Khieo Wildlife Sanctuary for their cooperation and permission to carry out this study. I am grateful to Andreas Koenig and Carola Borries, who developed the field site. I thank all the field assistants in Thailand for 
their help with data collection, especially Piya Saisawatdikul, Jureerat Wanart, Thawat Wisate and Kittisak Srithorn. ขอบคุณค่ะ!

The support from many colleagues made the completion of this thesis a lot easier. I thank Anja Ebenau for excellent collection of the data that we shared and for pushing me to my limit of working hours in the forest. I thank Miranda Swagemakers for her help in the field and for always bringing a smile to Thailand. Nadine Müller-Klein, I want to express my deepest gratitude to you for your continuous and patient support with statistics, revising my manuscripts and your positive attitude. Eva-Maria Rathke, you are such a wonderful colleague and friend, thank you for revising my manuscripts, sharing the Thai experience with "eleven elephants", the hours of discussions about life and millions of very important WhatsApp messages. Of course, I thank all my other colleagues in Göttingen, but I did not name everyone. I thank you all for the nice atmosphere in our department, the discussions about our data and sharing our frustration.

$\mathrm{Zu}$ guter Letzt gebührt meiner gesamten Familie mehr Ehrung, als ich jemals in Worte fassen könnte. Mama und Papa, danke für eure fortwährende, intensive Unterstützung seit ich denken kann. Danke für die zahlreichen Skype-Abende in Thailand und Deutschland, für die regelmäßigen Pakete und die Energie (wörtlich und im übertragenen Sinne), die ihr mir über Kontinente hinweg zukommen habt lassen. Ihr habt mir Flügel im thailändischen Wald und Reifen im deutschen Stadtgebiet verliehen. Ich liebe euch sehr. For a special person I should now probably switch to English again: Dear Timon, my native speaker, thank you for proof-reading and correcting all my manuscripts and sharing a passion for science with me. Ich danke meinen Großeltern Adolf, Bernhardine und Adeleh für die zahlreichen Proviant-Pakete, die mir nach Thailand geschickt wurden. Mimi und Tita, schön, dass ihr euch aufgrund der Entfernung dazu entschieden habt euch einen Computer zuzulegen und mit Skype auseinanderzusetzen. Ihr seid die coolsten Großeltern überhaupt. با تشكر از مامان عادله عزيزم براى حمايتش با بسته

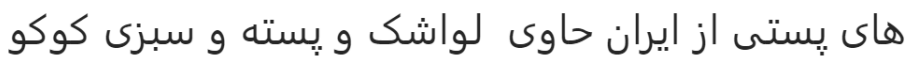

Marko, danke für deine unterstützenden Nachrichten während meiner Zeit im Feld. Danke für die schönen Erinnerungen an tiefgründige Unterhaltungen über Gott und die Welt im thailändischen Busch, und danke dir auch für die beständige Unterstützung seit ich in Deutschland lebe. Schön, dass wir unsere Leidenschaft für nicht-menschliche Primaten teilen. 


\section{Summary}

For zoologists, and especially primatologists, it has been a longstanding aim to decipher the causes of individual variability. Phenotypes associated with the hypothalamic-pituitary-adrenal (HPA)-axis, a central physiological pathway activated in response to stress, have been in the focus of research on wild primates. Scientists working on humans have gathered convincing evidence for a major role of genetics in explaining individual variation in HPA-axis-related phenotypes. Up to $50 \%$ and more of the variation in levels of the end product of the HPA-axis (cortisol), aggression and social behavior can be attributed to the underlying genotype. One non-synonymous substitution in a catecholamine degrader has been particularly well studied with regard to human aggression: $\mathrm{Val}^{158} \mathrm{Met}$ in the catechol-o-methyltransferase gene (COMT). Causing different activities of the enzyme that is substantially involved in catabolizing dopamine in the prefrontal cortex, the $\mathrm{Val}^{158}$ Met polymorphism has been repeatedly associated with aggressive behavior and has been assumed to be unique to humans probably due to species-specific cognitive abilities. Concerning the link between sociality and HPA-axis activity, first evidence from human literature indicates that the attenuating effect of social bonds on HPA-axis activity, a phenomenon called social buffering, is partly moderated by genetic variation.

The overall aim of this thesis was to promote and shed more light on the behavioral genetics of the primate HPA-axis under natural conditions. Studies linking genotype data to behavioral patterns in the wild are generally scarce and progress in this field has been hampered by a lack of convenient genetic high-throughput methods applicable to low-quality DNA samples. Further shortcomings concern that studies on non-human primates investigated only one or a few loci instead of screening several HPA-related target regions, broad-scale interspecific contrasts in behavioral patterns instead of actual observed individual behaviors and only one or two individuals of different primate species to conclude the absence of a polymorphism - as in the case of COMT Val ${ }^{158}$ Met.

In this thesis, I specifically addressed how genetic variation contributes to a better understanding of the following aspects of the primate HPA-axis: aggression rates (study 2), immunoreactive urinary cortisol levels, risk-taking, i.e. rate of initiating aggression, 
social bond strength, social bond maintenance and the social buffering effect (study 3 ). In order to carry out these behavioral genetic association studies, I firstly designed a multilocus next-generation sequencing panel including 46 target regions in 21 HPA-axis genes applicable to low-quality DNA samples (study 1).

I chose wild Assamese macaques (Macaca assamensis) as a study species, in which variation in cortisol levels and aggression as well as causal effects of social bonds have been previously demonstrated under natural selection pressures. In this species, the strength of male bonds predicts coalition formation and recruitment during fights, which in turn predict future social dominance relating to reproductive success. Data for this thesis were collected from adult male and female Assamese macaques living in four habituated groups in Phu Khieo Wildlife Sanctuary, Thailand. The non-invasive data collection covered standardized behavioral observations as well as the collection of fecal samples for DNA analyses and urine samples to quantify immunoreactive cortisol. Specifically, I designed a multi-locus sequencing panel (46 target regions in 21 genes, including COMT $\mathrm{Val}^{158} \mathrm{Met}$ ), genotyped all adults from four study groups (37 males, 39 females), collected focal animal behavioral data (5756 focal hours) and 366 urine samples from 23 males for quantification of immunoreactive cortisol via enzyme immunoassays.

The results of study 1 are (i) the compilation of a list of presumably functional target regions in genes that are involved in the HPA-axis, (ii) the design of a highthroughput genotyping panel useful when investigating the factors contributing to HPAaxis-related phenotypes, (iii) the demonstration that the panel is applicable to low-quality DNA samples such as feces, which is often the only available sample material from wild animal populations, and (iv) the demonstration that polymorphisms at purportedly functional HPA-axis loci exist in a natural primate population. By targeting 46 target regions in 21 genes 159 single nucleotide polymorphisms were detected.

Applying the genotyping panel designed in study 1, I identified the COMT $\mathrm{Val}^{158} \mathrm{Met}$ polymorphism and associated it with aggression rates of male and female macaques in study 2 . The results are that (i) the widely studied human COMT $\mathrm{Val}^{158} \mathrm{Met}$ polymorphism occurs in a non-human primate species with similar genotype frequencies (14 Met/Met, $40 \mathrm{Val} / \mathrm{Met}, 22 \mathrm{Val} / \mathrm{Val}$ ), (ii) macaques' aggression rates increased with dominance rank in Val/Val individuals, but decreased in individuals carrying other genotypes, and (iii) when changing from a lower to a higher dominance rank position, 
Val/Val individuals decreased, whereas Met/Met individuals increased their aggression rate.

In study 3 , I focused on 15 non-synonymous polymorphisms detected among the 159 single nucleotide polymorphisms and calculated a genetic risk score as the proportion of missense variants an individual carries. In the investigated males an increasing genetic risk score was associated with (i) increasing levels of immunoreactive urinary cortisol, (ii) decreasing risk-taking behavior, (iii) a decreasing social buffering effect, (iv) a trend to have stronger social bonds, and (v) an increasing maintenance of close proximity with strong partners.

These results contribute to our understanding of phenotypic consequences of individual variation in HPA-axis genotypes. Study 1 served as the basic methodology necessary to conduct study 2 and 3 and shall motivate field biologists to include multilocus genotype data in future studies on wild, non-model populations more frequently. As COMT $\mathrm{Val}^{158} \mathrm{Met}$ is not unique to humans and yields similar behavioral phenotypes in another primate species, follow-up studies on this polymorphism can be conducted in several species to investigate the distribution of this polymorphism among taxa and help to decipher its evolutionary roots and contribution to individual variation. The investigation of the social buffering hypothesis revealed a significant interaction effect between social bond strength and genetic risk on HPA-axis activity. The association between strong social bonds and low cortisol levels seemed to diminish when moving from low to high genetic risk, indicating that social buffering is in effect in individuals at the lower, but not the higher end of genetic risk. These results depict that future studies considering genotype as a mediator of social buffering in a wide range of animal taxa are essential. However, as the study included potential relatives, further analyses including relatedness data are necessary to decipher whether the associations between HPA-axis genotypes and phenotypes remain in effect when controlling for kinship.

In conclusion, I have performed the first comprehensive analysis of behavioral genetics associated with the HPA-axis in Assamese macaques. Combining ethological and molecular methods, my thesis suggests that genetics is a significant source of variability in a range of primate HPA-axis phenotypes. Thereby I was able to improve our knowledge of factors contributing to individual variation in HPA-axis activity, aggression rates, risk-taking, social bonding behavior and social buffering. Variation in the genetic 
constitution of macaques may allow individuals to adapt differently to social situations and stressors. Over evolutionary times the balance of different traits may preserve polymorphisms for different phenotypes in a population.

This thesis aids the advancement of multi-locus methods and the appreciation of wild animal populations in behavioral genetics. It broadens the spectrum of behavioral ecology and primatology in particular by addressing how inherent factors contribute to individual patterns and social mechanisms of animals in the wild. The fact that polymorphisms in HPA-axis genes cumulatively explain individual variation should have strong implications for primatological studies which in large parts neglected the genetic contribution to the investigated phenotypes. Primates can serve as valuable animal models which help to shed light on some of the ambiguous findings from human behavioral genetics by providing the chance to investigate naturalistic phenotypes using comparable measures. The incorporation of genotype in field primatology contributes to the current discussions about ambiguous findings in classical primatology and might have the potential to resolve some of them in the future. 


\section{Zusammenfassung}

\section{Das Zusammenspiel von HPA-Achsen-assoziierten Verhalten, Hormonen und Genen bei nicht-menschlichen Primaten}

Fragen nach den Ursachen interindividueller Variabilität beschäftigten häufig die in der Zoologie und insbesondere der Primatologie forschenden WissenschaftlerInnen. Eines der zentralen Forschungsgebiete in wildlebenden Primaten ist die Untersuchung von Phänotypen in Assoziation mit der Hypothalamus-Hypophysen-NebennierenrindenAchse (HPA-Achse). Diese Achse stellt einen physiologischen Signalweg dar, der in Reaktion auf einen Stressor aktiviert wird. In der Forschung an Menschen konnte gezeigt werden, dass bis zu $50 \%$ der individuellen Variabilität des Endprodukts der HPA-Achse (Cortisol), der Aggression und des sozialen Verhaltens durch genetische Faktoren erklärt werden können. Im Zusammenhang mit menschlichem Aggressionsverhalten ist $\mathrm{Val}^{158} \mathrm{Met}$, ein nicht-synonymer Polymorphismus im Catechol-O-Methyltransferase-Gen (COMT), besonders gründlich untersucht worden. Die Aminosäuresubstitution verändert die Enzymaktivität des Proteins, das wesentlich zum Abbau von Dopamin im präfrontalen Kortex beiträgt. Bisher wurde angenommen, dass dieser Polymorphismus nur in Menschen vorkommt. Bezüglich der Verbindung zwischen Sozialität und HPA-AchsenAktivität, konnten bei Menschen die mildernden Effekte von sozialer Unterstützung auf HPA-Achsen-Aktivität, die sogenannte soziale Abpufferung (,social buffering“), mit individueller genetischer Variation in Verbindung gebracht werden.

Die allgemeine Zielsetzung dieser Dissertation war es, das Verständnis der Verhaltensgenetik der HPA-Achse von Primaten in ihrem natürlichen Lebensraum zu erweitern. Ein Grund dafür, dass Forschung in diesem Gebiet bisher weitgehend vernachlässigt wurde, ist der Mangel an geeigneten genetischen Hochdurchsatzverfahren, die für DNA-Proben mit geringer Qualität anwendbar sind. Weitere bisherige Schwachpunkte finden sich unter anderem darin, dass vorhergehende Studien sich auf einige bzw. einige wenige Genorte beschränkten, anstatt mehrere HPA-Achsen-relevante Genorte parallel zu untersuchen, dass sich Studien auf Unterschiede in den generellen Verhaltensmustern zwischen Arten fokussierten, anstatt direkt beobachtetes Verhalten von Individuen zu analysieren, sowie, dass nur wenige Individuen in unterschiedlichen 
Primatenarten untersucht wurden, um auf die Inexistenz von Polymorphismen zu schließen, wie in dem Fall von COMT Val ${ }^{158}$ Met.

Spezifischer, beschäftigt sich diese Arbeit mit der Frage wie genetische Variationen mit den folgenden Aspekten der HPA-Achse in Verbindung stehen: Aggressionsrate (Studie 2), immunreaktiver Cortisolspiegel im Urin, risikoreiches Verhalten, i.e. die Rate mit der Aggression initiiert wird, Stärke und Aufrechterhaltung von Sozialbeziehungen, sowie soziale Abpufferung (Studie 3). Um diese Studien durchführen zu können, wurde zunächst ein Multilocus-Next-GenerationSequenzierungs-Panel für DNA-Proben mit geringer Qualität entwickelt (Studie 1).

Die Wahl der Spezies zur Untersuchung dieser Fragen fiel auf den AssamMakaken (Macaca assamensis), für den in früheren Studien Variationen in Cortisollevel und Aggression, sowie die kausalen Auswirkungen von Sozialbeziehungen bereits unter natürlichem Selektionsdruck gezeigt wurden. In dieser Spezies kann anhand der Stärke männlicher Sozialbeziehungen die Koalitionsbildung sowie die Rekrutierung von Unterstützern bei Auseinandersetzungen, welche letztlich Einfluss auf sozialen Rang und Reproduktionserfolg haben, vorhergesagt werden. Die für diese Dissertation gesammelten Daten stammen von adulten männlichen und weiblichen Assam-Makaken aus vier habituierten Gruppen im Phu Khieo Wildlife Sanctuary in Thailand. Die Datensammlung war rein nicht-invasiv und involvierte standardisierte Verhaltensbeobachtungen sowie das Sammeln von Kotproben für DNA-Analysen und Urinproben zur Quantifizierung von immureaktivem Cortisol in Urin. Im Rahmen der Dissertation wurden ein MultilocusNext-Generation-Sequenzierungs-Panel (46 Zielregionen in 21 HPA-Achsen-Genen, inklusive COMT $\left.\mathrm{Val}^{158} \mathrm{Met}\right)$ entwickelt, alle adulten Tiere der vier Gruppen (37 Männchen, 39 Weibchen) genotypisiert, Verhaltensdaten gesammelt (5756 Beobachtungsstunden), sowie 366 Urinproben von 23 Männchen für die Quantifizierung von immunreaktivem Cortisol mit Enzymassays gesammelt.

Die Ergebnisse der ersten Studie sind (i) die Zusammenstellung einer Liste von Zielregionen in Genen, die mit der HPA-Achse assoziiert sind, (ii) die Entwicklung eines Hochdurchsatz-Genotypisierungs-Panels für die Untersuchung von HPA-Achsenassoziierten Phänotypen, (iii) der Nachweis, dass das entwickelte Panel auch für DNAProben mit geringer Qualität, wie zum Beispiel Kotproben, anwendbar ist, sowie (iv) das Aufzeigen von vermutlich funktionalen Polymorphismen in HPA-Achsen-Genen in einer wildlebenden Primatenpopulation. 
Die Anwendung des in der ersten Studie entwickelten Genotypisierungs-Panels zeigte, dass der COMT $\mathrm{Val}^{158}$ Met Polymorphismus in Assam-Makaken existiert. Die Resultate der zweiten Studie sind, (i) dass der in Menschen intensiv untersuchte COMT Val ${ }^{158}$ Met Polymorphismus auch in einer nicht-menschlichen Primatenspezies mit einer ähnlichen Genotypfrequenz (14 Met/Met, $40 \mathrm{Val} / \mathrm{Met}, 22 \mathrm{Val} / \mathrm{Val}$ ) vorkommt, (ii) dass die Aggressionsrate in Val/Val-Individuen mit sozialem Rang zunahm, in Individuen mit anderen Genotypen jedoch abnahm und, (iii) dass in Val/Val-Individuen beim Wechsel von niedrigeren auf höhere Dominanzpositionen die Aggressionsrate abnahm, wobei diese bei Met/Met-Individuen zunahm.

Die dritte Studie fokussierte sich auf 15 nicht-synonyme Polymorphismen, anhand derer ein genetischer Risikowert als Proportion von Varianten die zu einem Aminosäureaustausch führen, kalkuliert wurde. Für die männlichen Makaken ergaben sich Assoziationen von einem zunehmenden genetischen Risikowert mit (i) zunehmenden immunreaktiven Cortisolspiegeln im Urin, (ii) abnehmendem risikoreichen Verhalten, (iii) einem abnehmendem sozialen Abpufferungseffekt, (iv) einer Tendenz für stärkere Sozialbeziehungen, sowie (v) zunehmende Aufrechterhaltung der räumlichen Nähe zu anderen Männchen mit einer starken Sozialbeziehung.

Diese Ergebnisse tragen zum Verständnis der Auswirkungen von individuellen Variationen in HPA-Achsen-Genen auf phänotypische Ausprägungen bei. Studie 1 stellte die für die Studien 2 und 3 notwendigen Methoden zur Verfügung und soll Feldbiologen dazu motivieren zukünftig häufiger Multilocus-Genotypisierung in verhaltensökologischen Studien an freilebenden Populationen in Betracht zu ziehen. Da nun gezeigt werden konnte, dass COMT Val ${ }^{158}$ Met keine einzigartige Besonderheit der Menschen ist, sondern auch in einer nicht-menschlichen Primatenart vorkommt und mit ähnlichen Verhaltensphänotypen assoziiert ist, kann in Folgestudien an verschiedenen Taxa dem Vorkommen und dem evolutionären Ursprung dieses Polymorphismus, sowie seinen Auswirkungen auf individuelle Variation nachgegangen werden. Die Untersuchung zur sozialen Abpufferung zeigte einen signifikanten Interaktionseffekt von der Stärke von Sozialbeziehungen und dem genetischen Risikowert auf die HPA-AchsenAktivität. Die Assoziation zwischen starken Sozialbeziehungen und niedrigen Cortisolwerten schien mit steigendem genetischen Risikowert schwächer zu werden und schließlich zu verschwinden. Das deutet darauf hin, dass der soziale Abpufferungseffekt in Individuen mit geringerem genetischen Risikowert in Kraft tritt, nicht aber in 
Individuen mit höherem genetischen Risikowert. Diese Ergebnisse unterstreichen die Relevanz, dass zukünftige Studien den Genotyp als moderierenden Faktor in Studien zur sozialen Abpufferung in verschiedenen Arten heranziehen. Um jedoch fundiertere Rückschlüsse über den Einfluss von Genotyp auf HPA-Achsen-assoziierte Phänotypen in Assam-Makaken ziehen zu können, sind weitere Analysen, in denen zusätzlich auf die Verwandtschaftsverhältnisse untersucht wird, notwendig, da diese Studie potentiell verwandte Individuen beinhaltete.

Die Forschungsresultate, welche sich im Rahmen dieser Dissertation ergaben, stellen die erste umfassende Analyse von Verhaltensgenetik in Verbindung mit der HPAAchse in Assam-Makaken dar. Die Kombination von Methoden aus der Verhaltungsforschung und der Molekularbiologie ermöglichte es in dieser Dissertation zu zeigen, dass Genetik eine signifikante Quelle individueller Variabilität in einer Reihe von HPA-Achsen-assoziierten Phänotypen bei Primaten ist. Dadurch konnten neue Erkenntnisse zu HPA-Achsen-Aktivität, Aggressionsraten, risikoreichem Verhalten, Sozialbeziehungen, sowie sozialer Abpufferung gewonnen werden. Genetische Variationen, die zu Variabilität in der HPA-Achsen-Aktivität in Makaken beitragen, könnten es Individuen ermöglichen sich unterschiedlich an soziale Umstände und Stressoren anzupassen. Über einen evolutionären Zeitrahmen hinweg könnte die ausgleichende Selektion verschiedener Merkmale dazu führen, dass Polymorphismen, die Individuen für unterschiedliche Phänotypen prädestinieren, erhalten bleiben.

Über den unmittelbaren Rahmen der Dissertation hinaus, unterstützt diese Arbeit die Weiterentwicklung von nicht-invasiven Multilocus-Genotypisierungs-Methoden und die Anerkennung von wildlebenden Tierpopulationen in der Forschung zur Verhaltensgenetik. Durch die Demonstration, dass inhärente Faktoren zu individuellen Mustern und sozialen Mechanismen in freilebenden Tieren beitragen, erweitert diese Dissertation das klassische Spektrum der Verhaltensökologie und besonders der Primatologie. Primaten stellen wichtige Tiermodelle dar in denen individuelle Variabilität unter natürlichen Bedingungen standardisiert erhoben werden kann. Daher kann verhaltensgenetische Forschung an wilden Primaten auch dazu beitragen die bisher wenig verstandenen Ergebnisse bei Menschen besser zu verstehen. Eine engere Einbindung von Genetik in der primatologischen Feldforschung hat großes Potential, um bei der Aufklärung von gegenwärtig zwiespältigen Befunden in der klassischen Primatologie mitzuwirken. 


\section{Chapter 1}

General introduction 


\subsection{Preface}

"One of the most dramatic developments in the behavioral sciences during the past few decades is the increasing recognition and appreciation of the important contribution of genetic factors to behavior" (Plomin et al., 2013, p. XVII). It is well recognized that genetics contributes fundamentally to phenotypes in humans and model organisms like mice. Popular examples come from various research fields, ranging from medical genetics to psychology. Even beyond the scientific community, it is appreciated that molecular advances contributed to e.g. prenatal diagnosis of genetic diseases (Wieacker \& Steinhard, 2010), the understanding of individual traits and the heritability of disorders, personality and intelligence (Plomin et al., 2016). Much of the research has been dedicated to examine the hypothalamic-pituitary-adrenal (HPA)-axis, a major neuroendocrine system which is essential for the regulation of many bodily functions in vertebrates (Charmandari et al., 2005; Munck et al., 1984). Variations in HPA-axis genes have been repeatedly associated with phenotypic variation in cortisol levels, aggression, sociality and mental disorders (e.g. Bolton et al., 2014; Bouma et al., 2012; Cases et al., 1995; Gogos et al., 1998; Gotlib et al., 2018; Higham et al., 2011; Inoue-Murayama et al., 2018; Jabbi et al., 2007; Papiol et al., 2007; Qayyum et al., 2015; Roy et al., 2010; Schatzberg et al., 2014; Schwandt et al., 2011). Among these variations, the COMT $\mathrm{Val}^{158} \mathrm{Met}$ polymorphism has received particular attention for its role in aggressive behavior and has been assumed to be unique to humans (Palmatier et al., 1999; Qayyum et al., 2015).

Behavioral genetics is an advanced field concerning model organisms. Mice can be genetically modified to develop virtually any symptom within specified time frames (e.g. Brown et al., 1996; Bruder et al., 2004; Morgan et al., 1996). The vast amount of evidence for genetic influences in model organisms might give the impression that mankind has mastered the genetic building blocks. Moving away from rodents, however, leads to a fast disillusionment: the genetic influences on phenotypic variation are not well explored in non-model organisms, especially in the wild. The genetic underpinnings of inter-individual differences are relatively understudied in field biology. 
One research area that particularly stands out due to long-term knowledge on individual animals and a strong interest to explain inter-individual differences is primatology. Among the main topics in research on our closest relatives is the investigation of individual variation in HPA-axis parameters. In particular, several studies have been devoted to decipher factors contributing to individuality in stress, aggression and sociality (e.g. Abbott et al., 2003; Anestis, 2005; Massen \& Koski, 2014; Morton et al., 2015; Muller \& Wrangham, 2004b; Rutberg \& Greenberg, 1990; Seyfarth et al., 2012, 2014; Young et al., 2014a). Combining social measures and the HPA-axis, the social buffering effect, whereby the presence of a close social partner attenuates stress responses of the HPA-axis, is a well-studied phenomenon in a multitude of social species (Cohen \& Wills, 1985; Edgar et al., 2015; Hennessy et al., 2009; Hostinar et al., 2014; Kiyokawa \& Hennessy, 2018; Wittig et al., 2016; Young et al., 2014a). Interestingly, preliminary evidence in humans indicates that genotype moderates the effectiveness of social bonds as a protective buffer against increased HPA-axis activity (Chen et al., 2011a).

While a general genetic contribution to several phenotypes is not under debate anymore, the consideration of genotypic predispositions to the primate HPA-axis and behavior is still scarce. The question "whether" genetic variation in HPA-axis genes contributes to phenotypes is settled in classical behavioral genetics and studies now focus on the questions "how and how much" of the phenotype is explained by genetic variation. In contrast, the "whether"-question has not yet been systematically implemented in many of the main research areas of field primatology, including the HPA-axis, aggression and social relationships. Currently there are fundamental gaps in our knowledge of whether and how HPA-axis-related phenotypes are influenced by genotype in wild primate populations because evidence is lacking or comes from studies on single gene variants in captivity. Most studies focused on one variant at a time (e.g. Barr et al., 2004; Miller et al., 2004; Newman et al., 2005; Pflüger et al., 2016), rather than screening several gene loci related to HPA-axis functioning (e.g. Ferguson et al., 2012), although the need for simultaneous investigations of multiple HPA-related genes has been voiced repeatedly (e.g. Bouma et al., 2012; Ferguson et al., 2012; Jabbi et al., 2007). Because the effects of single loci are likely to be small, studying genetic variants one at a time might lead to non-findings even in large sample sizes. Investigating multiple variants in parallel allows to cover multiple genes that are associated with a certain trait and integrate the small effects of many loci into one cumulative effect (Belsky \& Israel, 2014). 
This thesis sets out to give first insights about whether, how much of and how observed HPA-axis-related phenotypes can be explained by variation in HPA-axis genes. Focusing on this well-understood central physiological pathway, I investigated how genetic predispositions shape the natural phenotypic variation in behavior and cortisol levels in wild Assamese macaques (Macaca assamensis). Combining state-of-the-art genotyping methods for fecal samples, the quantification of immunoreactive cortisol from urine samples and detailed behavioral observations, in this thesis I specifically set out to: 1) contribute to methodological advances in this field via the design of a multi-locus genotyping panel to identify variants at 46 target regions in $21 \mathrm{HPA}$-axis genes; 2) inquire into the COMT $\mathrm{Val}^{158}$ Met polymorphism, its existence and association with aggression in a non-human primate; 3) combine the multi-locus data from the genotyping panel into one cumulative value, a so called genetic risk score, to predict individual HPA-axis activity and risk-taking behavior and to elaborate on the approach that genotype moderates the social buffering effect.

In the following introduction, I will present the general framework for my thesis by describing the main concepts which form the foundation of my work, outlining important findings but also shortcomings, and thereby explain the background and starting point of my project. At the end of the general introduction, I will present my study species, the Assamese macaque, and the general aims of my thesis and each study. 


\subsection{The hypothalamic-pituitary-adrenal-axis}

\subsubsection{An evolutionary conserved pathway}

The HPA-axis represents a central physiological pathway activated in response to stressors and is highly conserved across vertebrates (Schulkin, 2011). The stimulation of the HPA-axis initiates a cascade of neuroendocrine events, involving several signaling molecules and their respective receptors. While the types of signaling molecules vary among taxa, the physiological role of the HPA-axis in response to adverse stimuli is so fundamental that similar signaling pathways can be traced back to our vertebrate ancestors and even arthropods (Baker et al., 2007; Curran \& Chalasani, 2012; Laudet et al., 1992; Schulkin, 2011).

The anatomical structures of the HPA-axis are located in both the central nervous system and peripheral tissues. In vertebrates, the main elements of the HPA-axis are the hypothalamus, the pituitary gland and the adrenal glands. Hypophysiotropic neurons in the paraventricular nucleus of the hypothalamus are responsible for the synthesis and secretion of corticotropin-releasing hormone (CRH, Aguilera \& Liu, 2012). Under adverse circumstances they dispense $\mathrm{CRH}$, which is released into the portal circulatory system, by which it reaches its receptors on pituitary corticotropic cells (Bale et al., 2002; Drouin, 2011; Smith et al., 1998). The pituitary then releases adrenocorticotropic hormone $(\mathrm{ACTH})$ into the bloodstream, which stimulates the synthesis and secretion of glucocorticoids (GCs) from adrenocortical cells in the zona fasciculata of the adrenal cortex (Mesiano \& Jaffe, 1997; Simpson \& Waterman, 1988; Smith \& Vale, 2006). Eventually, GCs inhibit the secretion of ACTH and CRH, thereby creating a regulatory feedback loop of the HPA-axis (Axelrod \& Reisine, 1984; Bamberger et al., 1996; Charmandari et al., 2005; Everly \& Lating, 2013; Nicolson, 2008). The released GCs cause physiological effects when binding to their receptors, which are assumed to occur in all nucleated types of cells (Munck et al., 1984; Smith \& Vale, 2006). The predominantly occurring GCs vary among taxa (primates and fish - cortisol; rodents, birds, reptiles and amphibians - corticosterone), but all belong to the subclass of steroid hormones (Del Rey et al., 2008; Smith \& Vale, 2006). 
In addition to the corticoid pathway, whose main components are CRH (encoded by the $C R H$ gene) and its receptors (CRHR, CRHR1-2), GCs and their receptor (GCR, $N R 3 C 1)$, the $\mathrm{CRH}$ binding protein (CRHBP, CRHBP), corticosteroid binding globulin (CBG, SERPINA6), ACTH and its receptor (ACTHR, MC2R, Subbannayya et al., 2013), two other metabolic circuits mainly contribute to the HPA-axis: the serotonin and the catecholamine metabolism. Serotonin neurotransmission plays a role in the activation and the feedback loop of the HPA-axis by stimulating $\mathrm{CRH}, \mathrm{ACTH}$ and $\mathrm{GC}$ release and increasing the negative feedback control of GCs (Fuller, 1995; O'hara et al., 2007; Porter et al., 2004). The serotonin pathway involves serotonin (5-HT), its transporter (5-HTT, SERT, SLC6A4) as well as its receptor (5-HTR, HTR1-7), tryptophanhydroxylase (TPH, TPH1-2) and monoamino oxidase (MAO, MAOA-B, D'souza \& Craig, 2006). In response to activation by a stressor, neurons in the prefrontal cortex release catecholamines which induce the expression and release of CRH (Smith \& Vale, 2006). The catecholamine circuit includes dopamine (DA), its receptors (DRD, DRD1-4) and transporter (DAT, SLC6A3), and catechol-O-methyltransferase (COMT, COMT).

\subsubsection{The end product cortisol}

The activation of the HPA-axis culminates in the release of GCs (mainly cortisol in primates) from the adrenal cortex. This discovery has only been made in the last

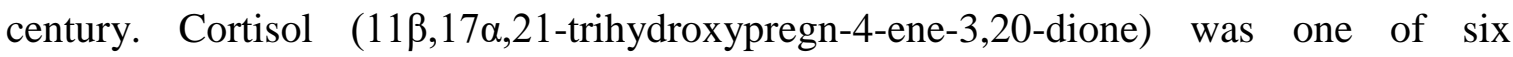
compounds that were isolated from adrenal glands in the 1930s by E. C. Kendall. Lacking any further knowledge about the functionality of these compounds, they were given the names A, B, C, D, E and F (later to be known as cortisol). Particularly compound E (later to be known as cortisone) received special attention by E. C. Kendall, T. Reichstein and P. S. Hench, who treated patients suffering from arthritis with this hormonally inactive cortisol metabolite. Kendall, Reichstein and Hench were jointly awarded the Nobel Prize in Physiology or Medicine in 1950 "for their discoveries relating to the hormones of the adrenal cortex, their structure and biological effects" (Nobel Media AB, 2014).

Like all adrenocortical steroid hormones, cortisol is synthetized from progesterone via three hydroxylation steps (Häggström et al., 2014; Kanehisa et al., 2016, 2017; 
Pasqualini et al., 1968). It is a metabolic hormone, inhibiting physiological processes which appear dispensable for an organism facing adverse situations (for example digestion, growth, immunity and reproduction) and allocating energy (glucose) to be used immediately (Charmandari et al., 2005; Munck et al., 1984; Nelson, 2011; Sapolsky et al., 2000). Thereby cortisol enables an organism to adjust its metabolic functions to meet the needs of the current situation in a changing environment (Nicolson, 2008).

The use of cortisol as a measure of HPA-axis activity is well established in human studies, using standardized social stress tests to elicit endocrine HPA-axis responses (von Dawans et al., 2011; Kirschbaum et al., 1993; Oldehinkel et al., 2011). Also in nonhuman primates and other animals GCs and their metabolites can be measured with high reliability, even from non-invasively collected samples and are therefore established endocrine markers (fish: Scott \& Ellis, 2007; birds: Goymann, 2005; rodents: Jansen et al., 2010; Nemeth et al., 2016; ruminants: Möstl et al., 2002; Palme \& Möstl, 1997; cats and dogs: Accorsi et al., 2008; Schatz \& Palme, 2001; primates: Bahr et al., 2000; Heistermann, 2010; Ostner et al., 2008a; diverse: Wasser et al., 2000). As the end product of the HPA-axis, cortisol is a promising candidate for a non-invasive and reliable measure of phenotypic variation in the HPA-axis. 


\subsection{Primate characteristics related to the HPA-axis}

\subsubsection{Aggression}

Besides the mere determination of cortisol levels, studies on the HPA-axis often also link HPA-axis activity to behavior, particularly aggression. The stimulation of brain areas associated with aggression results in increased GC levels (Halász et al., 2002; Kruk et al., 2004; Soma et al., 2008). Besides these findings from laboratory conditions, behavioral ecology studies have repeatedly associated aggression with GC levels in several taxa (fish: Øverli et al., 2002; birds: Carere et al., 2003; rodents: Huhman et al., 1991; ruminants: Salas et al., 2016; cats and dogs: Finkler \& Terkel, 2010; Rosado et al., 2010; primates: Cavigelli, 1999; Honess \& Marin, 2006; Ostner et al., 2008a). In wild chimpanzees, for example, participation in a single aggressive interaction causes GC levels to rise in both instigators and targets of aggression (Wittig et al., 2015).

Most aspects of aggression are currently adaptive or stem from strategies that were adaptive at some point in evolution (Wrangham, 2018). In the broadest sense, aggression serves the purpose to compete over and get access to certain resources, which can differ in their kind. Competition for food, mating partners, social status and space are among the most common scenarios (Buss \& Duntley, 2006; Georgiev et al., 2013). Therefore, aggression can be an important tool in intra- and intersexual as well as intraand interspecific competition. However, aggression is not a universally adaptive behavior. Rather it is adaptive when used in certain contexts and conditions, namely when the benefits of being aggressive outweigh its costs (Buss \& Duntley, 2006; Georgiev et al., 2013). The benefits of applying aggression to gain access to resources also depend on, amongst others, the value, abundance, spatial distribution and monopolization potential of the resources (Georgiev et al., 2013). Further, it has been suggested that genetic variability for aggression has been maintained by frequency-dependent selection (Smith et al., 1988). The simplest theoretical model that predicts genetic polymorphisms for aggression is probably the hawk-dove game (Smith et al., 1988). Smith and colleagues proposed three further game theory models (the war of attrition game, the size game and the badges of dominance game, Smith, 1974; Smith \& Brown, 1986, Smith et al., 1988), 
which also conclude that genetic variability for aggression could be maintained by frequency-dependent selection.

How aggression in measured differs according to research fields and the investigated species. Whereas mirror tests, attacking a dummy conspecific or an intruder are common ways to measure aggression particularly in captive animals (e.g. fish: Balzarini et al., 2014; Barlow et al., 1986; mice: Parmigiani, 1986; Roubertoux et al., 1999; macaques: Schwandt, et al., 2010), studies on humans often use questionnaires and ratings (e.g. Buss \& Durkee, 1957; Buss \& Perry, 1992; Yudofsky et al., 1986). In wild animal populations, natural aggressive behavioral patterns can be observed and quantified. Bite, slap, push and pull, lunge, threat, stare and chase are some of the most commonly included aggressive primate behaviors (e.g. Nelsen, 2017; Skinner \& Lockard, 1979). Both aggression and submission are important aspects in the negotiation of dominance hierarchies of primates (Deag, 1977; Nelsen, 2017) and other animal taxa. However, initiating such aggressive behaviors can bear costs and is therefore risky. These costs include physical (injury or death), physiological, psychological and energetic costs as well as an increased risk of predation and damage to social relationships (reviewed in Georgiev et al., 2013).

\subsubsection{Social relationships}

HPA-axis signaling is not only heavily involved in aggressive behavior but is also linked to affiliative behaviors and social bonding, especially in primates. Social bonds are formed when individuals bias affiliative interactions towards specific partners, leading to heterogeneity in interactions (Silk, 2002). By definition, a social bond is an affiliative relationship between two individuals, which is strong compared to other relationships in the group, equitable in their exchange of services and long-lasting (Ostner \& Schülke, 2014). Such affiliative relationships are established by repeated interactions over time and are a good predictor of future interactions (Cords, 1997; Seyfarth \& Cheney, 2012). Notably, studies on humans usually do not follow the above-mentioned definition of social bonds. Rather, the term social relationship is used for a wide range of sociality 
measures, including social network positions, questionnaires or self-reported ratings on social integration, loneliness and social support.

In contrast to human studies using questionnaires and reports to assess social behavior, field biologists have the opportunity to calculate measures from observed reallife interactions. In primates, grooming, fur-picking, or the manipulation of skin and hair by hand and mouth, not only has the hygienic function to remove ectoparasites, but also plays an important role in establishing and maintaining affiliative social bonds (Cooper \& Bernstein, 2000; Dunbar, 1991; Hutchins \& Barash, 1976; Tinklepaugh, 1931). Further, spatial proximity is one of the most widely used measures to quantify affiliative relationships in social groups of animals, which also allows comparisons between species (Whitehead \& Dufault, 1999). Being close to each other can be the result of individual partner preferences and increases the likelihood of social interactions (Altmann, 1965; Carpenter, 1945; Rowell \& Olson, 1983). The relative contributions of partners to the maintenance of proximity with one another can be assessed using the Hinde-index, which was originally invented to investigate mother-infant bonds (Hinde \& Atkinson, 1970). Several measures of affiliation can also be integrated into one value to assess specific aspects of social bonds, like bond strength - using the dyadic composite sociality index (CSI, Silk et al., 2003, 2006a).

Some non-human primate species form and maintain strong, equitable and stable relationship with individuals of the same and the opposite sex (e.g. Haunhorst et al., 2016; Kalbitz et al., 2016; Mitani, 2009; Ostner \& Schülke, 2014; Silk et al., 2010a). Partially, strong bonds can be explained by closeness in kinship, rank and age (e.g. Hamilton, 1964; Kalbitz et al., 2016; Kalbitzer et al., 2017; Mitani, 2009; Seyfarth \& Cheney, 2012; Silk et al., 2006a, 2010a). These relationships are assumed to build the basis for coalition formations in cooperative aggression, including recruitment for support from the audience and the likelihood of joining when being solicited (Schülke et al., 2010; Young et al., 2014b). Therefore, alliances, particularly in primates, are established before they are actually needed in coalitionary support (Dunbar, 2012; Harcourt, 1992).

In humans, social relationships influence mortality risk and health (e.g. Berkman \& Syme, 1979; House et al., 1982; for reviews see Berkman et al., 2000; Cacioppo \& Cacioppo, 2014; Cohen, 1988; Holt-Lunstad et al., 2010; House et al., 1988; Seeman, 1996; Uchino, 2004, 2006). The discovery that our closest relatives have similar social 
relationships like friendship in humans (e.g. Seyfarth \& Cheney, 2012; Silk, 2002), launched a cascade of studies on the consequences of close social bonds on well-being (Alberts, 2010), survival and longevity (Archie et al., 2014; Silk et al., 2010b; Thompson \& Cords, 2018), offspring survival (Silk et al., 2003, 2009), GC levels (Young et al., 2014a) and coalitionary support (Schülke et al., 2010; Young et al., 2014b).

Social buffering, a phenomenon explaining the positive association between social support and health, has been demonstrated in humans and other animals (Cohen \& Wills, 1985; Hennessy et al., 2009; Hostinar et al., 2014; Kikusui et al., 2006). Using mainly laboratory setups in humans and non-invasive methods under natural conditions in nonhuman primates, the activation of the HPA-axis has been a core variable investigated in conjunction with the social buffering hypothesis in both species (e.g. Chen et al., 2011a; Ditzen et al., 2007, 2008; Heinrichs et al., 2003; Kirschbaum et al., 1995; Wittig et al., 2016; Young et al., 2014a). In particular, the social buffering framework predicts that social bonds have a general attenuating effect on HPA-axis activity (main effect hypothesis) or social bonds have attenuating effects on HPA-axis activity only during stressful events (interaction effect hypothesis).

Social buffering probably depends on the adaptive value of a particular category of social relationships (Kiyokawa \& Hennessy, 2018), on the source and type of support (Thoits, 2011), and/or the nature of the relationship (Hennessy et al., 2009). Initial evidence for the functional importance of strong social bonds comes from the wild. In macaques, the strength of male bonds predicts coalition formation and recruitment during fights (Schülke et al., 2010; Young et al., 2014b). In turn, coalitions predict future social dominance which relates to reproductive success (Schülke et al., 2010).

As genetic variation might modulate the effectiveness of social bonds as a protective buffer against adverse effects (Chen et al., 2011a), it seems plausible that interindividual differences cause a significant variation in the social buffering effect. How genetic predisposition contributes to observed inter-individual variation in HPA-axis activity and the social buffering effect in non-human primates is still unknown. The genetic component of social buffering is not well investigated although its awareness could contribute to understand the underlying molecular mechanisms, divergent findings and individuals' health symptoms depending on differences in social support. Wild primates, for whom the ecological relevance and adaptive value of social bonds have been 
demonstrated under natural selection pressures, constitute an ideal system to start studying the behavioral genetics of social buffering. 


\subsection{Sources of inter-individual differences: introducing the era of behavioral genetics}

In non-human primates, individual differences in GC levels and aggression have been mainly associated with environmental factors, personality types, dominance rank and availability of social support (Abbott et al., 2003; Goymann et al., 2004; Muller \& Wrangham, 2004a; Sapolsky, 2005; Seyfarth et al., 2012; Young et al., 2014a). For example, female baboons show differences in their GC excretion levels and their social behavior according to their personality types (Seyfarth et al., 2012), the strength of social bonds influences the effects of cold stress and received aggression on GC levels (Young et al., 2014a), and social status has been related to measures of HPA-axis physiology both in a positive and negative way, including also non-findings (Abbott et al., 2003; Goymann et al., 2004; Sapolsky, 2005). Studies investigating individual patterns also reported inconsistent findings, with the potential effects of dominance rank probably representing the most prominent example from the non-human primate literature (Cavigelli \& Caruso, 2015). Meta-analyses have suggested that the associations between social status and GC levels might be resolved by incorporating the rate of being exposed to stressors, rank instability, opportunities for social support and allostatic load indices for individuals of high and low dominance rank, respectively (Abbott et al., 2003; Edes \& Crews, 2017; Goymann et al., 2004; Sapolsky, 1992, 2005).

The influence of an individual's genetic setup has received only little attention in studies aiming to explain individual variation in GC levels. Since we know from human studies that approximately $50 \%$ of the variation in cortisol levels and aggressive behavior can be explained by genetic make-up (Brendgen et al., 2006; Ferguson, 2010; Linkowski et al., 1993; Miles \& Carey, 1997; Rhee \& Waldman, 2002; Riese et al., 2009; Rietschel et al., 2017; Tucker-Drob et al., 2017; Tuvblad \& Baker, 2011; Young et al., 2000), this lack of attention on HPA-axis genetics in non-human primates is surprising. Most of the convincing evidence for the substantial effects of genotype on phenotypic variation was generated by human psychologists reaching into the discipline of genetics, creating the academic field of behavioral genetics. The most extensively studied topics in human behavioral genetics cover socially and clinically relevant issues such as aggression, stress and mental disorders, which are all tightly linked to the HPA-axis (e.g. Papiol et al., 2007; 
Varghese \& Brown, 2001). Hence, a large body of literature has been dedicated to the considerable genetic impacts on HPA-axis parameters to improve perspectives in clinical applications. As a critical component of translational research, a recent study proposes that social buffering works only in certain genotypes (Chen et al., 2011a). Performing standard social stress tests (Trier Social Stress Test, Kirschbaum et al., 1993) with 200 male participants and female supporters being present or absent, (Chen et al., 2011a) found that salivary cortisol responses to stress were lower after social support only in individuals carrying one or two copies of the $\mathrm{G}$ allele of the silent rs53576 polymorphism in the oxytocin receptor gene.

Besides endocrine aspects of the HPA-axis, also aggression has received much attention in behavioral genetics due to its relevance to society, including the risk to commit suicide (e.g. Baldessarini \& Hennen, 2004; Brent \& Mann, 2005; reviewed in Zai et al., 2012), domestic violence (e.g. Barnes et al., 2013), behavior of patients with psychiatric diseases (e.g. Han et al., 2004; Lachman et al., 1998; Strous et al., 1997, 2003) and criminality (e.g. Ferguson, 2010; Wasserman \& Wachbroit, 2001). Aggression is highly heritable, with up to $56 \%$ of the variation being explained by genotype in humans (Ferguson, 2010; Miles \& Carey, 1997; Rhee \& Waldman, 2002). In vervet monkeys (Chlorocebus aethiops) $61 \%$ of clear aggressive actions were attributed to genotype in a study on a captive population (Fairbanks et al., 2004).

Research on model organisms under laboratory conditions was motivated by early human findings in behavioral genetics, for example a case study of a Dutch family in which men expressed extreme aggressive outbursts (Brunner et al., 1993). This behavior has been linked to a deficiency of the MAOA gene, which is located on the $\mathrm{X}$ chromosome and thus affects preliminary males. Subsequently, MAOA knockout mice were investigated to confirm the genetic effects on observed aggressive behavior (Cases et al., 1995).

So far only a few studies on rhesus macaques (Macaca mulatta) linked HPA-axisrelated genes to physiological stress, aggression and sociality measures. In mostly experimental studies on captive individuals, it has been shown that polymorphisms in candidate genes such as the mu-opioid receptor (OPRM1), neuropeptide Y (NPY), CRH, TPH2, SLC6A4 and MAOA are associated with aggression, HPA-axis responses to stress, arousal during stress and grooming network parameters (Barr et al., 2004; Brent et al., 
2013; Ferguson et al., 2012; Lindell et al., 2010; Miller et al., 2004; Newman et al., 2005; Schwandt et al., 2011). Despite a body of research on the heritability of cortisol levels and aggression in Homo sapiens, how wild primates' genotypes contribute to HPA-axis phenotypes has not yet received much attention. At the same time, understanding the causes of large inter-individual variation has been one of the major goals of behavioral ecology in general and primatology in particular.

\subsubsection{Candidate genes and the single-locus approach: a focus on $C O M T$}

The candidate gene approach relies on existing knowledge about the physiological role of gene products and biological mechanisms underlying phenotypes. It is commonly applied in genetic studies on many organisms from plants to humans (Kwon \& Goate, 2000; Pflieger et al., 2001; Tabor et al., 2002; Zhu \& Zhao, 2007). The approach involves the identification of candidate genes that are most likely involved in a specific phenotype, the identification of variants within these genes, the genotyping of populations and the subsequent statistics to correlate variants with phenotypes (Tabor et al., 2002). Consistent replication can then be interpreted as a strong evidence of causality (Tabor et al., 2002).

Research has been successful in identifying genes involved in several phenotypic characteristics (Tabor et al., 2002). Advantages of the candidate approach are that genotyping is easy, relatively quick and inexpensive (Kwon \& Goate, 2000). However, this approach has been criticized due to non-replication and the impossibility to include all possible causative genes (Tabor et al., 2002; Zhu \& Zhao, 2007). It has also been argued that this pessimism is too extreme and that non-replication is probably due to differences in study designs, small effect sizes or real biological differences between populations (Tabor et al., 2002).

Candidate gene approaches are often a single-locus analysis. In this case, a promising candidate locus is selected and associated with the phenotype of interest, based on its functionality or prior knowledge from other association studies. In 2003, the most cited paper in the field of neuroscience was the first to demonstrate a gene-environment interaction of a single locus (Caspi et al., 2003). Individuals carrying a short allele of the 
SLC6A4 gene had a higher risk to develop depression if they experienced adverse life events (Caspi et al., 2003).

One of the most extensively studied candidate genes in human behavioral genetics is the COMT gene, which was identified in 1958 (Axelrod \& Tomchick, 1958; Dickinson \& Elvevåg, 2009). Especially studies focusing on stress and aggression, two topics with high societal relevance, often use COMT as a target region (Qayyum et al., 2015). COMT belongs to the catecholamine circuit of the HPA-axis in which it functions as a catalyst of catecholamines, such as dopamine (Axelrod \& Tomchick, 1958; Männistö \& Kaakkola, 1999), for which it is the main degrader in the prefrontal cortex (Käenmäki et al., 2010; Karoum et al., 1994; Matsumoto et al., 2003; Sesack et al., 1998; Yavich et al., 2007). It functions in two isoforms: as the membrane-bound (MB-COMT) and the soluble COMT (S-COMT), with MB-COMT being predominant in the central nervous system (Hong et al., 1998; Tenhunen et al., 1994).

High levels of catecholamines are associated with aggressive behavior (Coccaro et al., 1991; Puciłowski et al., 1986; Ratey \& Gordon, 1992; Tidey \& Miczek, 1996). Therefore, COMT, as a catecholamine catalyst, has been a major target in human studies on aggression. It was first suggested by Kuperman and colleagues (1988) that COMT is involved in aggressive behavior, when they correlated blood COMT levels of 31 men who were hyperactive during their childhood with measures of hostility and impulsiveness. Again, inspired by early findings from humans, a model organism was used to confirm the genetic effects on observed behavior. Male COMT knockout mice have higher levels of dopamine in the frontal cortex and are more aggressive (Gogos et al., 1998).

One specific locus in the COMT gene has attracted researchers' attention in particular: the $\mathrm{Val}^{158} \mathrm{Met}$ polymorphism (rs4680), a non-synonymous single nucleotide polymorphism (SNP) with a $\mathrm{G}$ to A base transition, causing an amino acid change from valine (Val) to methionine (Met). It is located in codon 158 of exon 4 of the COMT gene with the chromosome position 22q11.2 (Grossman et al., 1992). The Met allele of this polymorphism causes a $40 \%$ reduced activity of the COMT enzyme at body temperature (Chen et al., 2004), but up to three- to four-fold reduced activities have been reported (Lotta et al., 1995). This functionality stems from the protein surface, where Met has a lower hydrophoby than Val, thereby causing a lower enzyme stability (Chen et al., 2004; 
Liu \& Wang, 2003; Machius et al., 2003; Vidgren et al., 1994; Zubieta et al., 2001). This in turn leads to higher levels of dopamine in the prefrontal cortex of Met-carriers (Chen et al., 2004).

Due to the dopamine hypothesis of several psychiatric diseases, schizophrenia, attention-deficit hyperactivity disorder (ADHD) and borderline personality disorder have been in the focus of direct COMT effects, with carrying a Met-allele being a main predictor for the vulnerability to these mental disorders as well as aggression, homicide and suicide in schizophrenia patients (Han et al., 2006; Kia-Keating et al., 2007; Lazzaretti et al., 2013; Qayyum et al., 2015; Strous et al., 2003; Tadić et al., 2009; Tosato et al., 2011; Zai et al., 2012). The "warrior-worrier" model of the COMT Val ${ }^{158} \mathrm{Met}$ polymorphism suggests the maintenance of both alleles across human populations due to counterbalancing advantages and disadvantages in the Val-warriors and the Met-worriers (Goldman et al., 2005; Stein et al., 2006). They were given this designation because the warrior haplotype is associated with higher emotional resilience, higher stress resistance and better handling of pain, whereas the worrier haplotype is associated with a higher susceptibility to stress-related mental disorders, but also higher creativity and better cognitive skills (Goldman et al., 2005; Stein et al., 2006). Particularly regarding aggression, however, it has been suggested that the relationship between COMT $\mathrm{Val}^{158} \mathrm{Met}$ and phenotypic variation is not straightforward (Qayyum et al., 2015). Rather, COMT Val ${ }^{158}$ Met has been studied as a moderator between environmental factors such as stress and the severity of aggression (Hygen et al., 2015), impulsive aggression (Wagner et al., 2010), antisocial behavior (Thapar et al., 2005) and depression (Hosang et al., 2017).

In non-human primates, some polymorphisms orthologous to those in humans (e.g. length polymorphisms in SLC6A4 and MAOA) have been detected and linked to similar effects as in humans (Caspi et al., 2003; Inoue-Murayama, 2009; InoueMurayama et al., 2010; Newman et al., 2005; Wendland et al., 2006). Investigations also showed that not all corresponding regions are polymorphic in non-human primate species (Inoue-Murayama et al., 2010; Kalbitzer et al., 2016). The existence of COMT Val ${ }^{158} \mathrm{Met}$, one of the most extensively studied polymorphisms in human behavioral genetics, is still assumed to be unique to humans, probably due to species-specific cognitive abilities (Palmatier et al., 1999). This assumption is based on a study by Palmatier and colleagues 
(1999) who sequenced one gorilla, two chimpanzees, two bonobos and two orangutans, identified only the Val allele and concluded that this represents the ancestral state of the polymorphism which is not present in non-human primates. Today, a look at the NCBI GenBank database reveals that some macaque species carry the Val (Macaca fascicularis and nemestrina), whereas others carry the Met allele (Macaca mulatta and fuscata, Pflüger et al., 2016). Sequencing more than only a few representatives of a species might be necessary to detect genotypic variations.

\subsubsection{The multi-locus approach: a focus on genetic risk scores}

In contrast to single locus studies, the simultaneous investigation of multiple loci offers new ways to handle genotype data. One promising option, that is also easily adaptable to different approaches using small or large amounts of genotype information, is the calculation of a genetic risk score (GRS). Belsky and Israel (2014) refer to this new possibility as "the lowest hanging fruit and the most potentially disruptive to existing research programs" (Belsky \& Israel, 2014, p. 137).

GRSs are used in different sub-disciplines and have been referred to as genetic prediction score (Zhao et al., 2014), polygenic risk score (e.g. Llewellyn et al., 2014; Rietschel et al., 2017; Utge et al., 2018; Worley et al., 2015), allelic score (Spycher et al., 2012), multi-locus genetic profile (Feurer et al., 2017; Nikolova et al., 2011), SNP score (Vrieze et al., 2012), genotype score (Meigs et al., 2008) or genetic load (Ferguson et al., 2012). Compared to single locus approaches, the application of GRSs goes one step further and is a more sophisticated way to assess a dose-dependent relationship between genetic polymorphisms and phenotype. GRSs have been calculated from small-scale and large-scale genotype data, with a broad spectrum of the number of polymorphisms used (e.g. 4 in Worley et al., 2015 and Di Iorio et al., 2017; 6 in Utge et al., 2018; 7 in Ferguson et al., 2012; 9 in Kathiresan et al., 2008; 10 in Chen et al., 2011b; 13 in Ripatti et al., 2010; 116 in Morrison et al., 2007; thousands in e.g. Evans et al., 2009; Purcell et al., 2009; Wray et al., 2007). However, in all cases "the defining characteristic of a genetic risk score is that it provides a quantitative measure of genetic predisposition that 
is calculated using information from multiple genetic variants" (Belsky \& Israel, 2014, p. 142).

GRSs are a measure that summarizes an identified set of genetic variants into one cumulative value. This value can then be used to measure the aggregated influence of genotype on heterogeneity among individuals. The GRS provides information about an individual's position on the continuum of genetic risk (Belsky \& Israel, 2014). Because several genetic variants, including rare ones, contribute to most phenotypes, it is not possible to state where an individual lies on the continuum of genetic risk when knowing only one locus variant (Belsky \& Israel, 2014).

The application of GRSs has been given special attention in studies on humans aiming to assess the genetic risk for several diseases and brain functions, many of them calculating GRSs of the HPA-axis (e.g. diurnal cortisol levels: Utge et al., 2018; depression: Feurer et al., 2017; cardiovascular disease: Kathiresan et al., 2008; coronary heart disease: Morrison et al., 2007; Ripatti et al., 2010; obesity: Llewellyn et al., 2014; diabetes: Meigs et al., 2008; psoriasis: Chen et al., 2011b; asthma: Spycher et al., 2012; schizophrenia and bipolar disorder: Purcell et al., 2009; amygdala function: Di Iorio et al., 2017; ventral striatum reactivity: Nikolova et al., 2011; neurodevelopment: Worley et al., 2015). In a recent review, Belsky and Israel (2014) call out for a more frequent use of genetic approaches, especially GRSs, in studies on sociality. GRSs of HPA-axis genotypes have been referred to as an important marker for stress reactivity (Feurer et al., 2017). Ferguson and colleagues (2012) were among the first to investigate more than one candidate locus simultaneously in a non-human primate model species, the rhesus macaque. They calculated a cumulative risk value (genetic load) from three loci and associated it with HPA-axis dysregulation in captive individuals. In a more recent study, Madlon-Kay and colleagues (2018) found only weak effects of 12 SNPs in the oxytocin and arginine vasopressin receptor genes on social behavior in a population of rhesus macaques on Cayo Santiago island. In their study they focused on single variant associations and did not calculate an integrated value from all detected polymorphisms. Also, including variants from the broader gene network contributing to the complex behaviors of interest might be necessary to detect the small effects of many loci that cumulatively contribute to phenotypes. Regarding these examples from the literature, no comparable studies have been conducted with primates living in their natural habitat so 
far. What is lacking in particular is a holistic approach combining polymorphisms in genes coding for elements in all of the three HPA-axis metabolisms with naturally occurring phenotypic variation under natural conditions. In this thesis I aimed to close this gap using extensive genetic, behavioral and endocrine data collected from a wild primate species, the Assamese macaque. 


\subsection{Study species: the Assamese macaque (Macaca assamensis)}

Kingdom Animalia

Phylum Chordata

Class Mammalia

Order Primates

Suborder Haplorrhini

Parvorder Catarrhini

Superfamily Cercopithecoidea

Family Cercopithecidae

Subfamily Cercopithecinae

Tribe Papionini

Genus Macaca

Species Macaca assamensis

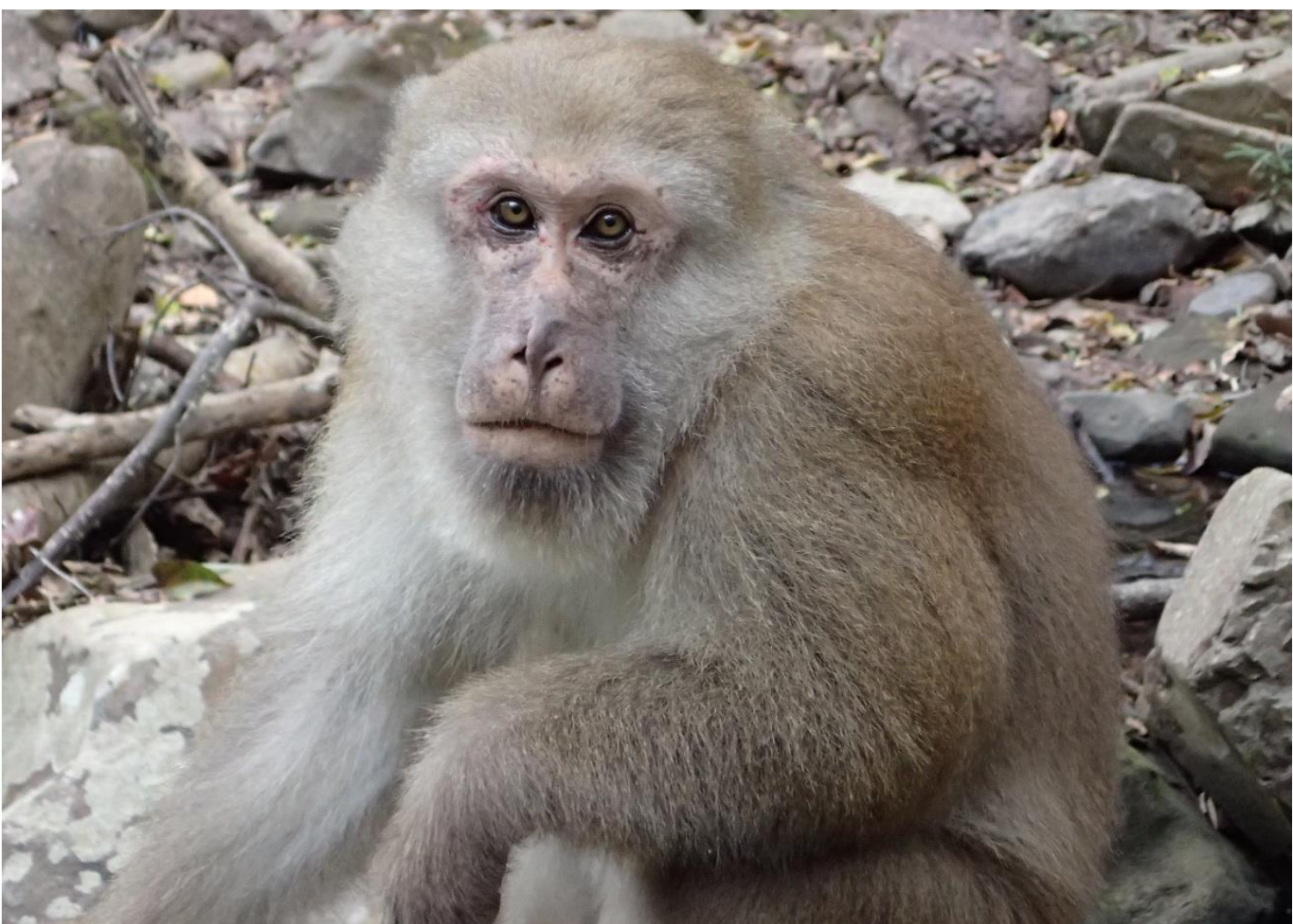

Figure 1.1: An adult male Assamese macaque in Phu Khieo Wildlife Sanctuary, Thailand. Picture by Daria R. Gutleb. 


\subsubsection{General background}

Macaques (Macaca spp.) are monophyletic and diverged from a common ancestor with humans approximately 25 - 28 million years ago (Kumar \& Hedges, 1998; Morales \& Melnick, 1998; Rogers \& Gibbs, 2014). The genus name Macaca derives from the word "ma-kako", meaning "monkey" in a Congolese Bantu language ('macaca', 2000). Except for humans, macaques have the largest geographic range among primates and are among the best-studied taxa (Thierry et al., 2004). Paleontological findings from the Pleistocene show that macaques existed from North Africa, through Europe to Asia (Abegg \& Thierry, 2002; Delson, 1980; Eudey, 1980; Fa, 1989). Today, only one relict species inhabits Northern Africa, the Barbary macaque (Macaca sylvanus), and the other 22 known macaque species all occur only in Asia. The present macaques can be divided into seven species groups: sylvanus, silenus, Sulawesi macaques, sinica, fascicularis, mulatta and arctoides (Zinner et al., 2013).

The study species in this thesis is the Assamese macaque (Macaca assamensis MCCLELLAND 1839, Figure 1.1) which is a member of the sinica group of macaques, next to Macaca sinica, radiata and thibetana (Fooden, 1980; Zinner et al., 2013). Assamese macaques can be divided into two sub-species, the Eastern (or Southeast Asian) Assamese macaque (Macaca assamensis assamensis) and the Western (or subHimalayan) Assamese macaque (Macaca assamensis pelops Hodgson 1840). With a currently decreasing population trend, both sub-species are classified as near threatened according to The IUCN Red List of Threatened Species (The IUCN Red List of Threatened Species, 2017). Habitat degradation and poachers constitute the main threat (The IUCN Red List of Threatened Species, 2017).

The population that was investigated in this thesis belongs to the Eastern Assamese macaque, which occurs between 200 and $2750 \mathrm{~m}$ above sea level in China, Tibet, India, Myanmar, Thailand, Laos and Vietnam (Brandon-Jones et al., 2004; Eudey, 2013; Fooden, 1982). This brownish colored macaque has habitats in subtropical and tropical, evergreen and semi-evergreen, deciduous and mixed bamboo, wet and dry, lowland and mountainous forests (Eudey, 2013). Assamese macaques are sexually dimorphic, with males being larger (head-body $53-73 \mathrm{~cm}$ ) and heavier $(8-17 \mathrm{~kg})$ than 
females (17 - 29 cm, 5 - 9 kg, Eudey, 2013; Fooden, 1982). They are mainly vegetarian, but the diet also includes insects and small vertebrates such as lizards and squirrels (Eudey, 2013; Koirala et al., 2017; Mitra, 2002; Schülke et al., 2011; Zhou et al., 2011). Like in many macaque species, there exist groups that are provisioned, raid crops or beg for food from tourists (Aggimarangsee, 1992; Chalise, 2003; Eudey, 2013; Fooden, 1982; Kaewpanus et al., 2015; Sarkar, 2014b). In captivity this primate can reach the age of approximately 28 years (Dathe, 1983; Eudey, 2013).

In the following section, I will describe those features of Assamese macaques which appear relevant for the studies that I conducted in this thesis: their dominance hierarchy, social behavior and seasonality. Afterwards, I will introduce the population of Assamese macaques that has been studied for this thesis: four wild groups living in their natural habitat in Phu Khieo Wildlife Sanctuary (PKWS) in Thailand.

\subsubsection{Hierarchy, sociality and seasonality}

Like all macaque species, Assamese macaques live in multimale-multifemale groups with males being the dispersing sex (Thierry et al., 2004). Females usually stay within their natal group lifelong, therefore matrilineal kin plays a particularly important role in female social relationships (Thierry et al., 2004).

Macaques can be classified into four grades according to the strictness of the female dominance hierarchy, aggression and conflict management (Thierry, 2000; Thierry et al., 2008). Nepotism and the asymmetry of agonistic outcomes decline, whereas reconciliatory behavior increases from grade 1 to 4 . Assamese macaques are classified as grade 2 macaques with a strict linear steep dominance hierarchy (Bernstein \& Cooper, 1999; Cooper \& Bernstein, 2008; Macdonald et al., 2013; Ostner et al., 2008a, 2011). Female macaques acquire ranks close to their mothers, which is referred to as a "classical nepotistic hierarchy" or a "classical matrilineal dominance structure" (Chapais, 2004). Therefore, a female's rank position is quite stable and predictable from knowledge on her kinship. Males disperse at the age of puberty, but can continue to migrate between groups later in life (van Noordwijk \& van Schaik, 2004; Thierry, 2007). Also, they can 
change their dominance rank position throughout their life (Schülke et al., 2010). To acquire a higher rank, male-male coalitions are of particular importance (Schülke et al., 2010). Males who have strong social bonds form coalitions, which in turn predict future dominance and reproductive success (Schülke et al., 2010).

Assamese macaques may allocate up to almost a quarter (10 - $24 \%)$ of their daily time for social activities, depending on food availability (Chalise, 1999; Koirala et al., 2017; Pandey \& Chalise, 2015; Paudel \& Chalise, 2017; Sarkar et al., 2012; Sarkar, 2014b, 2014a). Female Assamese macaques groom other females and juveniles more often than males do (Cooper \& Bernstein, 2000), and reconcile more often with strong grooming partners (Cooper et al., 2005). Importantly, male Assamese macaques form strong and stable social bonds with females (Haunhorst et al., 2016; Ostner et al., 2013), but also males (Kalbitz et al., 2016, 2017). In primates, social bonds among males are generally rare and male Assamese macaques stand out in this regard (Ostner \& Schülke, 2014).

Assamese macaques are seasonal breeders with a distinct birth/non-mating season from March to September and a mating season from October to February (Fooden, 1971; Fürtbauer et al., 2010; Mitra \& Alfred, 2007). The mating season is characterized by distinct behavioral and physiological changes. Males show increased levels of aggression, GCs and androgens (Ostner et al., 2008a, 2011). During the winter months, females have concealed ovulations, synchronize mating, show distinct promiscuity and initiate $70 \%$ of all copulations (Fürtbauer et al., 2010, 2011a, 2011b). Females gestate 5.5 months and give birth every 1 to 2 years (Fürtbauer et al., 2010). Due to the behavioral and hormonal changes between the non-mating and the mating period, any study investigating long-term data must take the season when data were collected into account. 


\subsubsection{Assamese macaques in Phu Khieo Wildlife Sanctuary}

The study population in PKWS ranges in the area of Huai Mai Sot Yai $\left(16^{\circ} 5^{\prime}-\right.$ $35^{\prime} \mathrm{N}, 101^{\circ} 20^{\prime}-55^{\prime} \mathrm{E}, 600-800 \mathrm{~m}$ above sea level) in the province of Chaiyaphum in Isan, the north-eastern part of Thailand. With an $1543 \mathrm{~km}^{2}$ area of predominantly undisturbed dry evergreen and hill evergreen forest with bamboo patches, PKWS is a major part of the $6500 \mathrm{~km}^{2}$ protected Western Isaan Forest Complex (Borries et al., 2002; Grassman et al., 2005). The research field site in PKWS was established by A. König and C. Borries, who firstly described the diurnal primate community in PKWS (Borries et al., 2002) and studied the socio-ecology of Phayre's leaf monkeys (Trachypithecus phayrei, Borries et al., 2004; Koenig et al., 2004a, 2004b). Besides Assamese macaques, the sanctuary harbors the following seven primate species: white-handed gibbons (Hylobates lar), Phayre's and silvered leaf monkeys (Trachypithecus cristatus), slow loris (Nycticebus coucang), stump-tailed (Macaca arctoides), pig-tailed (Macaca nemestrina) and rhesus macaques (Borries et al., 2002).

Since 2005 research on the study population of wild Assamese macaques in their natural habitat in PKWS is directed by J. Ostner and O. Schülke. It began with the effort to habituate first one, then two groups of Assamese macaques (AS and AO). The groups split in 2012 and 2014, respectively, thus four groups have been observed since then (ASM, ASS, AOM and AOS). Due to natural and frequent male migrations, groups experience a natural genetic flow (in contrast to laboratory or semi-free groups of primates). Also, one rare case of an immigrated female was observed. Only the researchers within the long-term project and occasionally rangers have access to the study groups, tourists are not allowed to enter the forest. Students continuously collect data following standardized observation and sampling protocols to assure the usability of data for long-term analysis. They do not interact with or feed the monkeys and try to keep a minimum distance of $5 \mathrm{~m}$ to observe natural behavior without disturbance.

As partially described above, the study population exhibits a strong seasonality in reproduction (Fürtbauer et al., 2010, 2013) and males react to the challenges of the mating season with a pronounced increase in aggression rates and GC output (Ostner et al. 2008a, 2011). Males can be ordered along a linear, relatively steep dominance 
hierarchy (Ostner et al. 2008a) and individual paternity success increases with increasing dominance rank (Schülke et al. 2010; Sukmak et al. 2014a). Further, previous studies on the wild Assamese macaques in PKWS included research on their ecology (Schülke et al., 2011), females' mating synchrony and concealed fertility (Fürtbauer et al., 2011a, 2011b), feeding competition (Heesen et al., 2013, 2014, 2015), immature development (Berghänel et al., 2015, 2016), molecular analyses of mitochondrial DNA, microsatellites and adenoviruses from non-invasively collected fecal samples (Müller et al., 2014; Sukmak et al., 2014b, 2017), male-male, male-female and male-infant relationships (Haunhorst et al., 2016, 2017, Kalbitz et al., 2016, 2017; Minge et al., 2016; Ostner et al., 2013; Ostner $\&$ Schülke, 2018) and other aspects of their sociality (Macdonald et al., 2013; Richter et al., 2016; Schülke et al., 2010, 2014).

Regarding this range of research topics, the behavioral genetics approach in this thesis is new not only regarding the lack of genetic studies on wild primate behavior, but also a new approach in the long-term field project in PKWS. Considering that individual variation in GC levels and aggression as well as the causal connection between male social bonds and reproductive success have been previously demonstrated in this population of Assamese macaques (Ostner et al., 2008a; Schülke et al., 2010), they serve as ideal study animals to investigate the genetic underpinnings of behavior and hormones associated with the primate HPA-axis. Due to research on wild primates living in a protected area, all studies on the Assamese macaques of PKWS are of completely noninvasive nature. For my studies, I therefore collected fecal samples for non-invasive genetic analyses and urine samples for non-invasive hormone analyses from all four currently observed groups. 


\subsection{Aims and approaches: studying genetic influences on phenotypes associated with the HPA-axis in a natural primate population}

Inter-individual differences have been in the focus of research since the very beginning of primatology. One source of the observed endocrine and behavioral variation might be genotypic differences. To corroborate this hypothesis, studies investigating whether and how wild primates' HPA-axis activity, aggressive behavior and social relationships are affected by genetic predispositions are necessary. By digging deeper into the behavioral genetics and physiogenetics of wild animals, we will gain a better understanding of the factors that contribute to variation within populations under natural selection pressures and shed light on the proximate mechanisms affecting individuals' physiological and behavioral states. The formation of strong social bonds and their causal effects on reproductive success have been previously demonstrated in wild Assamese macaques (Kalbitz et al., 2016; Schülke et al., 2010) in which it is possible to renounce from experimental physiological and behavioral manipulations and directly observe and quantify the natural range of social behavior and HPA-axis activity.

In this thesis, I investigated how genetic predispositions shape the behavior and physiology of wild Assamese macaques. I focused on the effects of HPA-axis-related genetic variants on aggression and HPA-axis activity. Although non-invasive molecular techniques have been optimized over the past decades, the integration of genotype in HPA-axis-related research on non-human primates and even more so on primates living in their natural habitat with a natural level of genetic variation is still rare. Studies linking genotype data, e.g. MAOA and SLC6A4, to behavioral patterns are scarce, often investigate only single candidate genes rather than screening several gene loci related to HPA-axis functioning (e.g. Miller et al., 2004; Schwandt et al., 2011) and use broad-scale interspecific contrasts in behavioral patterns instead of actual observed individual behaviors (Chakraborty et al., 2010; Wendland et al., 2006).

Using non-invasive genetic methods, I took the consequent next step following the current state-of-the-art in research on inter-individual differences in aggression, HPA-axis activity, social bonds and social buffering effects in wild non-human primates. I collected behavioral and endocrine data across an entire year, thus including both a non-mating and the subsequent mating period of this seasonal species. Combining detailed data on 
behavior with molecular analyses of cortisol and identification of genetic variants, I aimed to provide new perspectives on the connections between HPA-axis-related phenotypes and genetic make-up and how observed phenotypic variation can be explained by inherent genetic predisposition. Variants in the genetic constitution of macaques may allow individuals to adapt differently to social situations or constraint them in their behavior, thereby causing the observed inter-individual variation that might reflect different strategic approaches.

We lack methods to easily genotype individuals for several gene loci simultaneously using low-quality fecal DNA samples. Therefore, the first study (chapter 2) of my thesis was dedicated to design a genotyping panel for a fast and simultaneous identification of multiple HPA-axis gene loci. By reviewing the literature, I compiled a list of candidate gene loci that were targeted in this study. The target loci included genes coding for elements of all three metabolic circuits of the HPA-axis, thus including a key set of proteins that are involved in HPA-axis-functioning. The designed genotyping panel enabled me to determine the variation at 46 target regions in 21 HPA-axis-related genes of all adult males and adult females in the study population and constitutes the basic methodology for the further two studies that I conducted.

Applying the panel from study 1 (chapter 2), I found out that the extensively studied COMT $\mathrm{Val}^{158} \mathrm{Met}$ polymorphism also exists in the study population of wild Assamese macaques. In study 2 (chapter 3), I report on the existence of the polymorphism in a species other than the human and investigate the association between COMT genotype and aggression in adult males and females, considering a moderating role of the polymorphism between rates of aggressive behavior and dominance rank.

In study 3 (chapter 4), I simultaneously investigate all identified SNPs from the genotyping panel in study 1 (chapter 2) and calculate a GRS as the proportion of missense variants an individual carries. I test whether GRS of HPA-axis-related genes predicts urinary immunoreactive cortisol levels, risk-taking behavior, i.e. initiating aggression, social bonding and the main social buffering effect in adult male Assamese macaques.

I summarize the results from the chapters 2,3 and 4 at the beginning of the general discussion (chapter 5). Furthermore, I discuss the relevance of my studies and give prospects for future research. 


\section{Chapter 2}

\section{Study 1: Non-invasive genotyping with a massively parallel sequencing panel for the detection of SNPs in HPA-axis genes}

D. R. Gutleb ${ }^{1,2,3^{*}}$, J. Ostner ${ }^{1,2,3}$, O. Schülke ${ }^{1,2,3}$, W. Wajjwalku ${ }^{4}$, M. Sukmak , C. Roos $^{5,6}$, A. Noll ${ }^{6}$

${ }^{1}$ Department of Behavioral Ecology, Johann-Friedrich-Blumenbach Institute for Zoology and Anthropology, University of Goettingen, Göttingen, Germany

${ }^{2}$ Research Group Social Evolution in Primates, German Primate Center, Leibniz Institute for Primate Research, Göttingen, Germany

${ }^{3}$ Leibniz ScienceCampus Primate Cognition, Göttingen, Germany

4 Department of Farm Resources and Production Medicine, Faculty of Veterinary Medicine, Kasetsart University, Nakhon Pathom, Thailand

${ }^{5}$ Gene Bank of Primates, German Primate Center, Leibniz Institute for Primate Research, Göttingen, Germany

6 Primate Genetics Laboratory, German Primate Center, Leibniz Institute for Primate Research, Göttingen, Germany

published in Scientific Reports

2018;8:15944

doi: $10.1038 / \mathrm{s} 41598-018-34223-y$

submitted 26.02.2018, revised 23.07.2018 \& 3.10.2018, accepted 8.10.2018 


\section{Abstract}

We designed a genotyping panel for the investigation of the genetic underpinnings of inter-individual differences in aggression and the physiological stress response. The panel builds on single nucleotide polymorphisms (SNPs) in genes involved in the three subsystems of the hypothalamic-pituitary-adrenal (HPA)-axis: the catecholamine, serotonin and corticoid metabolism. To promote the pipeline for use with wild animal populations, we used non-invasively collected faecal samples from a wild population of Assamese macaques (Macaca assamensis). We targeted loci of 46 previously reported SNPs in 21 candidate genes coding for elements of the HPA-axis and amplified and sequenced them using next-generation Illumina sequencing technology. We compared multiple bioinformatics pipelines for variant calling and variant effect prediction. Based on this strategy and the application of different quality thresholds, we identified up to 159 SNPs with different types of predicted functional effects among our natural study population. This study provides a massively parallel sequencing panel that will facilitate integrating large-scale SNP data into behavioural and physiological studies. Such a multifaceted approach will promote understanding of flexibility and constraints of animal behaviour and hormone physiology. 


\section{Introduction}

Recent developments in molecular techniques enable researchers to include largescale investigations of genetic impacts on behavioural or endocrine variables at reasonable costs (Ekblom \& Galindo, 2011; Perry, 2014). In studies on humans, the investigation of genotypic influences on phenotypic characteristics revealed that interindividual variation can be strongly affected by genotype (Craig \& Halton, 2009; Pavlov et al., 2012; Plomin et al., 2013). For example, genotypic variation is responsible for approximately $50 \%$ of inter-individual variation in physiological stress levels and aggression (Linkowski et al., 1993; Miles \& Carey, 1997). In animal studies, however, the consideration of underlying genotype in behavioural and physiological studies is relatively understudied. Especially regarding studies on wild, non-model populations, several authors have called for a more frequent consideration of genetic impacts on animal behaviour (Brent \& Melin, 2014; Ekblom \& Galindo, 2011; Tung et al., 2010). In this study, we provide a multi-locus genotyping pipeline, based on non-invasively sampled material from a population of wild primates, facilitating future research on social and ecological factors driving variation in stress and aggression.

Modulation of the hypothalamic-pituitary-adrenal (HPA)-axis activity is an effective mechanism mediating environmental effects on the organism including its behavioural tendencies. The HPA-axis is a central physiological pathway activated in response to stress and is conserved across vertebrates (Denver, 2009; Schulkin, 2011). In behavioural ecological studies, aggressive behaviour is often linked to HPA-axis activity via quantification of cortisol, the end product of this pathway (fish: Øverli et al., 2002; birds: Carere et al., 2003; rodents: Huhman et al., 1991; ruminants: Salas et al., 2016; cats and dogs: Finkler \& Terkel, 2010; Rosado et al., 2010; primates: Honess \& Marin, 2006; Ostner et al., 2008b). Links to behaviour have been established in rats where the stimulation of brain areas responsible for aggression causes cortisol release, and similar processes are proposed for other vertebrates, including humans (Halász et al., 2002; Kruk et al., 2004). Behavioural ecological studies on aggression often assess how the expression of aggressive behaviour is related to social dominance or affected by characteristics of the competitive situation without conceptually integrating interindividual variation due to genetic variation. 
Three main metabolic circuits contribute to the HPA-axis: the serotonin, the catecholamine and the corticoid. Genes associated with these metabolic circuits have been repeatedly targeted in human clinical stress (Jabbi et al., 2007; Zhou et al., 2008) and aggression (Craig, 2007; Pavlov et al., 2012; Qayyum et al., 2015) research. Functional polymorphisms in the genes coding for the three subsystems may lead to dysregulations in the HPA-axis pathway and a change in how the organism reacts to external stressors. The serotonin pathway involves the serotonin transporter (SLC6A4), receptor (HTR), tryptophan hydroxylase (TPH) and monoamine oxidase (D'souza \& Craig, 2006). The neuropeptide Y (NPY) is a neurochemical that plays a protective role in stress resilience (Kormos \& Gaszner, 2013; Zhou et al., 2008). The catecholamine circuit (e.g. dopamine: DRD, SLC6A3, catechol-O-methyl transferase: COMT) causes general physiological changes that prepare the body for physical activity (Molinoff \& Axelrod, 1971). Main components of the corticoid pathway are the corticotropin-releasing hormone (CRH), $\mathrm{CRH}$ receptors (CRHR), the glucocorticoid receptor (NR3C1), $\mathrm{CRH}$ binding protein (CRHBP), corticosteroid binding globulin (SERPINA6) and the corticotropin receptor (MC2R, Subbannayya et al., 2013).

For human diagnostics in the field of behavioural genetics, studies acquire large datasets via high-throughput methods such as massively parallel sequencing (i.e. nextgeneration sequencing, Perry, 2014). Behavioural studies on natural animal populations, however, commonly target one or a few gene loci associated with aggression and HPAaxis activity (Garamszegi et al., 2014; Kalbitzer et al., 2016; Timm et al., 2015), whereas high-throughput multi-locus approaches are rather rare (but see Bergey et al., 2016; Ekblom \& Galindo, 2011). Due to the high number of genes involved in physiological pathways such as the HPA-axis, the simultaneous assessment of multiple loci known to affect certain traits promises a much more comprehensive understanding of the investigated physiological and behavioural parameters (Ferguson et al., 2012; Pearce et al., 2017). The introduction of massively parallel sequencing technologies makes a multilocus approach also feasible in studies on non-model species. The generated data provide high coverage of amplicons or genomes and a large and still growing body of different bioinformatics applications helps to investigate multiple loci in a fast and parallel way (e.g. Genome Analysis Toolkit - GATK, McKenna et al., 2010; SAMtools, Li et al., 2009 and UCSC genome browser, Kent et al., 2002). 
Here we report a massively parallel sequencing panel for the assessment of HPArelated SNPs useful for studies investigating the genetics that underlie behavioural and endocrine variation in aggression and the physiological stress response. For this purpose, we targeted loci of previously reported SNPs in 21 candidate genes associated with the HPA-axis. We provide detailed information on all steps from selection of target genes and polymorphisms, via laboratory work to the subsequent bioinformatics analyses of acquired massively parallel sequencing data. We additionally demonstrate the feasibility of application to faecal samples from wild populations, where non-invasive sampling is necessary. 


\section{Materials and Methods}

\section{Ethical statement}

The National Research Council of Thailand (NRCT) and the Department of National Parks, Wildlife and Plant Conservation (DNP) approved (permit numbers: $0004.3 / 3618,0002.3 / 2647,0002 / 17,0002 / 626,0002 / 2424)$ the data collection at the study site in Thailand and export to Germany as part of a long-term collaboration between the University of Goettingen, the German Primate Center, the DNP and Kasetsart University Bangkok under the agreement of benefit sharing. Faecal samples were collected non-invasively. No animals were harmed or sacrificed for this study. Procedures were in accordance with the American Society of Primatologists' (ASP) principles for the ethical treatment of primates (https://www.asp.org/society/resolutions/EthicalTreatmentOfNonHumanPrimates.cfm).

\section{Sample collection and storage}

Samples were collected at Phu Khieo Wildlife Sanctuary in north-eastern Thailand. The sanctuary is part of the $6,500 \mathrm{~km} 2$ protected Western Isaan Forest Complex. Faecal samples were collected from four groups of fully habituated and individually identifiable Assamese macaques. In total, we collected 478 faecal samples from 38 adult males and 41 adult females (1-15 per individual, $\varnothing 6$ ) over the course of the long-term field project between June 2006 and January 2016. $58 \%$ of the samples were collected between March 2015 and January 2016. Adult males are involved in reproduction, have fully developed testes and long canines. Females are considered as adult in the mating season that they first conceive in, dating back from observations of their first birth,

For genetic analyses $\sim 5 \mathrm{~g}$ of faeces were collected immediately after defecation from the surface of the faecal sample from an identified individual. We applied the two- 
step storage procedure, which included the collection of faecal samples into $50 \mathrm{ml}$ tubes (62.559.001, Sarstedt, Nümbrecht, North Rhine-Westphalia, Germany) containing $30 \mathrm{ml}$ of $97 \%$ ethanol (Nsubuga et al., 2004). After storage for 24 to 36 hours, ethanol was poured off and the faecal samples were dried and stored on $30 \mathrm{ml}$ silica beads (11292600-2, Intereducation Supplies Co., Ltd., Bangkok, Thailand) in $50 \mathrm{ml}$ tubes in the dark at room temperature (Roeder et al., 2004). These samples were exported to Germany within 6 months and then stored at $-20^{\circ} \mathrm{C}$ until DNA extraction was performed.

\section{SNP selection and amplicon primer design}

Target regions were determined by searching the literature for SNPs in candidate genes involved in stress and aggression. The majority of target regions were chosen from literature on humans (for references see Supplementary Table S1.1), but we also targeted macaque and pig SNPs (for full list see Supplementary Table S1.1). Further, we chose only target regions located in protein-coding genes that code for receptors, enzymes and transmitter molecules associated with the HPA-axis. Targets were located both in exonic and intronic as well as untranslated regions. In total, we selected 46 target regions in 21 candidate genes. A summary about all genes that were included in the multi-locus genotyping panel can be found in Table 2.1, for more details about the target regions, including chromosomal position, SNPs, functional consequences and selected references see Supplementary Table S1.1.

As genome data for our study species, the Assamese macaque (Macaca assamensis), is not available, we designed primers according to the genome sequence of the congeneric rhesus macaque (Macaca mulatta, v8.0.1). For detailed information about amplicon sequences of Macaca mulatta and human see Supplementary Table S1.1.

Primers were designed using the online-software Primer3Plus (Untergasser et al., 2012). Due to DNA degradation in faecal samples, primers were designed to amplify short PCR products with a maximum of $380 \mathrm{bp}$ ( $\varnothing 207 \mathrm{bp}$ ), with the target SNP being as 
Table 2.1: List of all genes included in the multi-locus genotyping panel

\begin{tabular}{|c|c|c|}
\hline $\begin{array}{l}\text { HGNC gene } \\
\text { symbol }\end{array}$ & Name & Information \\
\hline AVPR1B & $\begin{array}{l}\text { arginine vasopressin } \\
\text { receptor } 1 \mathrm{~B}\end{array}$ & $\begin{array}{l}\text { - present in the brain as well as in the pituitary where it } \\
\text { stimulates ACTH release } \\
\text { - responsible for mediating the effects of vasopressin on } \\
\text { ACTH release } \\
\text { - inactivation reduces aggression }\end{array}$ \\
\hline $\mathrm{BDNF}$ & $\begin{array}{l}\text { brain-derived } \\
\text { neurotrophic factor }\end{array}$ & $\begin{array}{l}\text { - associated with stress vulnerability } \\
\text { - involved in emotion processing and cognition } \\
\text { - 'neurotrophic hypothesis' states that stress reduces BDNF } \\
\text { activity resulting in decreased function within limbic brain } \\
\text { regions }\end{array}$ \\
\hline COMT & $\begin{array}{l}\text { catechol-O-methyl } \\
\text { transferase }\end{array}$ & $\begin{array}{l}\text { - degrades catecholamines such as dopamine, epinephrine } \\
\text { and norepinephrine } \\
\text { - due to its function in catecholamine degradation and } \\
\text { dopamine inactivation it plays a pivotal role in neuro- } \\
\text { cerebral stress processing } \\
\text { - essential neuropeptide for maintenance of homeostasis } \\
\text { - functional gene variants associated with enzyme activity, } \\
\text { cortisol levels and aggression }\end{array}$ \\
\hline $\mathrm{CRH}$ & $\begin{array}{l}\text { corticotropin-releasing } \\
\text { hormone }\end{array}$ & $\begin{array}{l}\text { - plays a crucial role in the behavioural and neuroendocrine } \\
\text { stress response } \\
\text { - dysregulations are linked to stress-related psychiatric } \\
\text { disorders } \\
\text { - experimental manipulations demonstrated that naturally } \\
\text { occurring gene variants mediate individual variability in } \\
\text { behavioural and physiological traits, thus determining an } \\
\text { individual's coping style }\end{array}$ \\
\hline CRHBP & $\begin{array}{l}\text { corticotropin releasing } \\
\text { hormone binding protein }\end{array}$ & $\begin{array}{l}\text { - this high-affinity protein regulates CRH availability } \\
\text { - widely distributed throughout the body } \\
\text { - variation in CRHBP expression influences the } \\
\text { effectiveness of CRH in stimulating ACTH to release } \\
\text { cortisol }\end{array}$ \\
\hline CRHR1 & $\begin{array}{l}\text { corticotropin-releasing } \\
\text { hormone receptor } 1\end{array}$ & $\begin{array}{l}\text { - binds CRH } \\
\text { - important for endocrine and behavioural stress response } \\
\text { - polymorphisms predict baseline cortisol and stress-related } \\
\text { psychotic disorders }\end{array}$ \\
\hline CRHR2 & $\begin{array}{l}\text { corticotropin-releasing } \\
\text { hormone receptor } 2\end{array}$ & - binds CRH in the membranes of hormone-sensitive cells \\
\hline DRD3 & dopamine receptor D3 & $\begin{array}{l}\text { - expressed in phylogenetically old regions of the brain } \\
\text { - thus plays a role in cognitive and emotional functions } \\
\text { - allelic variants are associated with aggression and } \\
\text { personality }\end{array}$ \\
\hline FKBP5 & FK506 binding protein 5 & $\begin{array}{l}\text { - is a co-chaperone of the glucocorticoid receptor } \\
\text { - together they form a complex that modulates cortisol } \\
\text { binding affinity } \\
\text { - thus, it modulates the HPA-axis via glucocorticoid } \\
\text { receptor sensitivity } \\
\text { - gene variants associated with baseline cortisol }\end{array}$ \\
\hline HTR1A & serotonin receptor $1 \mathrm{~A}$ & $\begin{array}{l}\text { - binds serotonin } \\
\text { - activation induces the secretion of hormones including } \\
\text { cortisol, corticosterone, ACTH and oxytocin } \\
\text { - important for stress-related information processing } \\
\text { - effects on the cortisol stress response which are explained } \\
\text { by differences in serotonin turnover } \\
\text { - level of activation is associated with aggression }\end{array}$ \\
\hline
\end{tabular}




\begin{tabular}{|c|c|c|}
\hline HTR1B & serotonin receptor 1B & $\begin{array}{l}\text { - widely expressed in the central nervous system } \\
\text { - function depends on location, in frontal cortex: } \\
\text { postsynaptic receptor and inhibits dopamine release, in } \\
\text { ganglia and striatum: inhibits serotonin release } \\
\text { - gene variants associated with vulnerability for depression } \\
\text { and anxiety }\end{array}$ \\
\hline MAOA & monoamine oxidase $\mathrm{A}$ & $\begin{array}{l}\text { - breaks down serotonin, melatonin, noradrenaline and } \\
\text { adrenaline } \\
\text { - therefore, it influences synaptic concentrations of these } \\
\text { neurotransmitters } \\
\text { - marker for antisocial behaviour } \\
\text { - gene variants linked to aggression and impulsivity in } \\
\text { animals and humans }\end{array}$ \\
\hline MC2R & ACTH receptor & $\begin{array}{l}\text { - binding of ACTH stimulates cortisol production } \\
\text { - mutations cause familial glucocorticoid deficiency }\end{array}$ \\
\hline NPY & neuropeptide $\mathrm{Y}$ & $\begin{array}{l}\text { - most abundant neuropeptide in the mammalian brain and } \\
\text { affects its activity } \\
\text { - plays an important role in controlling physiological } \\
\text { processes associated with stress, especially via emotion } \\
\text { - released in response to stress } \\
\text { - referred to as a protective neurochemical that mediates } \\
\text { stress resilience }\end{array}$ \\
\hline NR3C1 & glucocorticoid receptor & $\begin{array}{l}\text { - cortisol and other glucocorticoids bind to this receptor } \\
\text { - expressed in almost every cell of the body } \\
\text { - regulates gene transcription } \\
\text { - gene variants are associated with cortisol levels and } \\
\text { psychosocial stress and can cause hypersensitivity to } \\
\text { glucocorticoids and a poor feedback regulation of the } \\
\text { HPA-axis }\end{array}$ \\
\hline OPRM1 & opioid receptor mu 1 & $\begin{array}{l}\text { - high affinity for enkephalins and beta-endorphin } \\
\text { - exists mostly presynaptically } \\
\text { - gene variants associated with different endorphin affinities } \\
\text { - in macaques a variant influences HPA-axis function in } \\
\text { response to a variety of stressors }\end{array}$ \\
\hline OXTR & oxytocin receptor & $\begin{array}{l}\text { - } \text { presence in central nervous system } \\
\text { - modulates stress, anxiety, social memory and sexual, } \\
\text { aggressive and affiliative behaviours }\end{array}$ \\
\hline SERPINA6 & $\begin{array}{l}\text { corticosteroid binding } \\
\text { globulin }\end{array}$ & $\begin{array}{l}\text { - binding and transport of glucocorticoids in mammals } \\
\text { - assumed that polymorphisms affect glucocorticoid } \\
\text { transport efficiency }\end{array}$ \\
\hline SLC6A3 & dopamine transporter & $\begin{array}{l}\text { - actively removes neurotransmitters from the synaptic cleft } \\
\text { - thereby regulates the synaptic availability of dopamine } \\
\text { and the duration of dopaminergic neurotransmission } \\
\text { - in macaques a variant is associated with dominance rank }\end{array}$ \\
\hline SLC6A4 & serotonin transporter & $\begin{array}{l}\text { - regulates the serotonin re-uptake in the synaptic cleft } \\
\text { - since effects of serotonin in the synapse are terminated by } \\
\text { re-uptake, it is a crucial protein to regulate serotonin } \\
\text { function in the brain } \\
\text { - functional gene variants in humans and macaques have } \\
\text { been associated with several behavioural phenotypes }\end{array}$ \\
\hline TPH2 & tryptophan hydroxylase 2 & $\begin{array}{l}\text { - it is the rate-limiting enzyme in the synthesis of serotonin } \\
\text { - thus, responsible for the synthesis of serotonin in brain } \\
\text { areas } \\
\text { - associated with plasma cortisol }\end{array}$ \\
\hline
\end{tabular}

Note: For more details about the target regions, including chromosomal position, SNPs, functional consequences and selected references see Supplementary Table S1.1. 
central as possible. Primer annealing temperatures were between 54 and $60{ }^{\circ} \mathrm{C}$, with a maximum difference of $2{ }^{\circ} \mathrm{C}$ for each primer pair. Primer annealing temperature was chosen as predicted by Netprimer (Premier Biosoft, Palo Alto, California, USA). Further steps of the primer design included: (i) specificity check: Primer-Blast, NCBI, 'nr' database for Mammalia (Altschul et al., 1990), (ii) dimerization check: Netprimer (Premier Biosoft, Palo Alto, California, USA), (iii) secondary structure check: The mfold Web Server, DNA Folding Form (Zuker, 2003). The designed primers were ordered from Metabion (Planegg/Steinkirchen, Bavaria, Germany). In total we designed 41 primer pairs for 46 target loci (Supplementary Table S1.1).

\section{Laboratory methods}

DNA extraction was carried out with the First-DNA all-tissue Kit (D1002000, GEN-IAL GmbH, Troisdorf, North Rhine-Westphalia, Germany), following the manufacturer's protocol for DNA extraction from faeces. The protocol included an overnight incubation at $37{ }^{\circ} \mathrm{C}$ with lysis buffer 1 , lysis buffer 2 and proteinase K. After centrifugation, the supernatant was combined with lysis buffer 3 , incubated at $-20{ }^{\circ} \mathrm{C}$ for 5 minutes and centrifuged, followed by washing steps with $70 \%$ ethanol stored at -20 ${ }^{\circ} \mathrm{C}$. Finally, DNA was eluted in $50 \mu \mathrm{l}$ HPLC water (115333, Merck, Darmstadt, Hesse, Germany) and stored at $-20{ }^{\circ} \mathrm{C}$ until further processing. All steps of the protocol were carried out with DNA LoBind Tubes (0030108051, Eppendorf AG, Hamburg, Germany). Total genomic DNA concentration was measured with a NanoDrop Spectrophotometer (ND-1000, PEQLAB Biotechnologie GmbH, Erlangen, Bavaria, Germany) and diluted to a final concentration of $100 \mathrm{ng} / \mu \mathrm{l} .78 \%$ of the extracted faecal samples were collected between March 2015 and January 2016.

Target regions were amplified in 96-well plates (AB0600, Thermo Fisher Scientific, Waltham, Massachusetts, USA) with 1 U BioThermTaq DNA Polymerase (GC-002-5000, Genecraft, Cologne, North-Rhine Westphalia, Germany) in a $30 \mu 1$ PCR mix $(1 \times$ reaction buffer, $0.16 \mathrm{mM}$ for each $\mathrm{dNTP}, 0.33 \mu \mathrm{M}$ for each primer, and $18 \mathrm{ng}$ BSA, 100 ng template DNA), with the following thermocycler (Labcycler, Sensoquest, Göttingen, Lower Saxony, Germany) conditions: 2 minutes at $94^{\circ} \mathrm{C}, 60$ cycles of 30 
seconds at $94^{\circ} \mathrm{C}, 30$ seconds at the appropriate annealing temperature (see Supplementary Table S1.1), 30 seconds at $72^{\circ} \mathrm{C}$, and 5 minutes at $72^{\circ} \mathrm{C}$. To check for PCR contamination, we ran 3 to 7 non-template controls on each 96-well plate. After amplification, aliquots were size-separated on $2 \%$ agarose gels along with a size standard (SM0241, Thermo Fisher Scientific, Waltham, Massachusetts, USA) to check for PCR performance and correct amplicon size. PCR products were then purified with Solid Phase Reverse Immobilization (SPRI) technology using 2.5x Ampure Beads (A63881, Beckman Coulter, Brea, California, USA) and again subjected to $2 \%$ agarose gel electrophoresis to control for purification performance. DNA concentration was measured with a Qubit 3.0 (Q32854, Thermo Fisher Scientific, Waltham, Massachusetts, USA). To test if our primers are target-specific, all 41 SPRI-purified amplicons of two individuals, acquired via PCR from faecal DNA extracts, were sequenced using Sanger technology. Therefore, we applied both amplification primers $(3.3$ pmol $)$ and the Big Dye Cycle Sequencing Kit (433776452, Thermo Fisher Scientific, Waltham, Massachusetts, USA), and ran the reactions on an ABI 3130xl genetic analyzer (Thermo Fisher Scientific, Waltham, Massachusetts, USA). Sequence electropherograms were checked with DNA Baser (DNA Sequence Assembler v4, 2013, Heracle Biosoft S.R.L, Mioveni, Argeș, Romania) and compared with the respective target sequences of rhesus macaque and human.

For massively parallel sequencing, the amplicons from each individual were pooled in equimolar amounts to a total of $120 \mathrm{ng}$. Sequencing libraries were generated following the method described in Rohland et al. (2015) without uracil-DNA-glycosylase treatment based on Meyer and Kircher (2010) and Kircher et al. (2012). To check for performance of library preparation, we ran all libraries on an Agilent 2100 Bioanalyzer (Agilent Technologies, Santa Clara, California, USA) using the High Sensitivity DNA chip (5067-4626). Libraries were then pooled to a library mix with a final concentration of $10 \mathrm{nM}$. We added an additional step to control for quantity by running a qPCR with a 7500 Fast Real-Time PCR System (Thermo Fisher Scientific, Waltham, Massachusetts, USA) using the Bio-Rad EvaGreen Supermix (1725211, Bio-Rad, Hercules, California, USA) following the manufacturer's recommendations and using three samples of the library mix and the concentration standards $(5 \mathrm{nM}, 10 \mathrm{nM}, 20 \mathrm{nM})$. Reactions were run under the following conditions: 2 minutes at $50{ }^{\circ} \mathrm{C}, 10$ minutes at $95{ }^{\circ} \mathrm{C}, 40$ cycles of 30 seconds at $95{ }^{\circ} \mathrm{C}, 34$ seconds at $60{ }^{\circ} \mathrm{C}$ and 30 seconds at $72{ }^{\circ} \mathrm{C}$. Sequencing was 
conducted on an Illumina MiSeq sequencer (paired-end $150 \mathrm{bp}$ ) at the Microarray and Deep-Sequencing Core Facility, University Medical Center Goettingen, Lower Saxony, Germany.

\section{SNP calling}

After Illumina sequencing, all produced FASTQ reads were quality-checked and trimmed with FastQC (Andrews, 2010) and Trimmomatic v0.36 (Bolger et al., 2014). For SNP calling, we used the GATK best practices pipeline for germline SNP discovery (Auwera et al., 2013) as well as the SAMtools-bcftools-pipeline (Li et al., 2009). For both pipelines, all quality-checked reads were mapped against the genome of Macaca mulatta v8.0.1 using BWA MEM v0.7.12 ( $\mathrm{Li}, 2013)$. We followed the GATK best practice pipeline and did not mark duplicates in the bam-file, because it is not possible to distinguish duplicate reads in amplicon sequencing where the major proportion of sequences reads represents PCR duplicates (Ebbert et al., 2016; Tata et al., 2017). A first variant call was carried out using GATK's HaplotypeCaller. Recovered SNPs were filtered using the hard-filtering procedures recommended by GATK's best practices (VariantFiltration). The following quality filter expression was applied: quality by depth smaller than 2.0 (QD < 2.0), mapping quality smaller than 40.0 (MQ < 40.0), Fisher strand (Phred-scaled p-value using Fisher's Exact Test) more than 60.0 (FS > 60.0), mapping quality rank sum (the u-based z-approximation from the Mann-Whitney Rank Sum Test for mapping qualities) smaller than -12.5 (MQRankSum $<-12.5)$, and read position rank sum (the u-based z-approximation from the Mann-Whitney Rank Sum Test for the distance from the end of the read for reads with the alternate allele) smaller than 8.0 (ReadPosRankSum < -8.0)50. Afterwards, Base Quality Score Recalibration (BQSR) was performed twice using GATK's BaseRecalibrator and PrintReads. The final variant calling process was conducted with GATK's HaplotypeCaller in GVCF mode. The produced GVCF-files were merged using GATK's GenotypeGVCFs.

SAMtools v1.4 mpileup was also used to generate raw variant calls using Macaca mulatta v8.0.1 as reference genome. The following settings were used: -d (maximal perfile depth) set to 250 , -E (recalculate BAQ), --BCF (generate genotype likelihoods in 
BCF format), and --output-tags set to DP [to get the DP (number of filtered reads covering the corresponding allele) tag in the output file]. Variant calling was done using bcftools call v1.4 with the following settings: --multiallelic-caller (alternative model for multiallelic and rare-variant calling) --variants-only (output variant sites only) -O v (output type: 'v' uncompressed VCF, Li et al., 2009).

Only variants identified by both the GATK and the SAMtools pipeline lying within the ranges of the genes of interest were used for subsequent analyses. Additionally, we compared the number of variants called without a threshold for the Phred quality (QUAL) score, with QUAL set to be $\geq 30$, and with QUAL set to be $\geq 100$. The Phred quality score gives a logarithmically related prediction-value to the base-calling error. The higher the quality score, the higher the base call accuracy (Ewing et al., 1998).

\section{Variant annotation and effect prediction}

SnpEff v4.3i (Cingolani et al., 2012) and Variant Effect Predictor v87 (VEP, McLaren et al., 2016) were used for variant annotation and SNP effect prediction. We compared two different applications because variant prediction software can differ in their predicted effects (Wertz et al., 2016). With the VEP plugin 'MaxEntScan' it was possible to compare scores of the reference and mutant splice sites using a maximum entropy model and to predict splice site effects. Additionally, linkage disequilibrium scores were calculated using vcftools v0.1.1458 with the option 'geno-r2'. Subsequently, all calculated annotations and effects were analysed in detail by hand.

\section{Data accessibility}

Massively parallel sequencing-data were submitted to the Sequence Read Archive (SRA) available via NCBI with the accession number SRP116685. Additional data are available in the Supplementary Information. 


\section{Results}

Sanger sequencing of the 41 amplicons from two macaque individuals revealed that the applied primers are target-specific. From the 79 investigated macaque individuals, we obtained a total of 3066 amplicons, with a minimum of 34 and a maximum of 41 amplicons per individual (Ø 38.6). For 10 of the 79 individuals, the first faecal DNA extracts (3 samples collected in 2007 and 7 samples collected in 2015) did not yield any amplicons (probably due to inhibitors in faeces), however, amplicons were successfully obtained from second extracts derived from other samples from the same individuals in all cases.

After sequencing on Illumina's MiSeq, we obtained a total of 23,604,930 reads. Around $95 \%$ of the reads exhibit a Phred score $>29$. About $85 \%$ of the reads could be mapped against the reference genome of Macaca mulatta v8.0.1 with a mean depth of 3,219.81 and a mean mapping quality of 59.02. Detailed sequencing statistics can be found in Supplementary Tables S1.2 and S1.3.

\section{Variant calling}

We compared different variant calling approaches in order to use only those variants reproduced by multiple pipelines. Using the complete dataset of shared variants without consideration of any filtering steps (QUAL, QD, MQ, FS, MQRankSum, and ReadPosRankSum), $70.12 \%$ of the variants called by SAMtools were also found with the GATK pipeline, whereas only $28.31 \%$ of the variants called by GATK were also found by SAMtools (Supplementary Figure S1.1). In sum, SAMtools called 230 SNPs, whereas GATK detected 373 SNPs. In total, 169 SNPs in 21 genes were verified using both variant calling approaches. 10 out of the 169 SNPs showed homozygosity for the alternate allele and thus represented simply a difference to the rhesus macaque genome, whereas 159 SNPs were identified as polymorphic sites within the study population. General 
descriptive statistics of the distribution of individuals being homozygous for the reference or alternate allele, or heterozygous, can be found in Table 2.2.

Using the described filtering steps without consideration of the QUAL scores, the number of detected variants by GATK decreased to 170 SNPs resulting in 144 SNPs in 20 genes shared by both callers. Using different QUAL scores as further selection steps, the number of detected polymorphisms changed again. Extracting only those variants with a QUAL score $\geq 30$, GATK still detected 170 SNPs, but SAMtools called only 194 SNPs (Supplementary Figure S1.1). Only 140 SNPs in 20 genes were shared by both callers. With a QUAL score $\geq 100$, the number of variants decreased further to 124 shared SNPs in 20 genes out of 170 SNPs detected with GATK and 162 SNPs detected with SAMtools (Supplementary Figure S1.1).

\section{Variant effect prediction}

The variants detected by both the SAMtools and the GATK pipeline were used to predict possible variant effects. For this purpose, we used two applications: VEP and SnpEff. Comparing the results, almost all variants were classified as the same consequence type in both applications. The only differences are two counts of ' 5 '-UTR premature start codon gain variant' found with SnpEff, but not with VEP and 25 additional 'intergenic variants' found by SnpEff (Table 2.3). Consequently, the predicted variant effects show a high similarity between both methods concerning their impact (Table 2.4).

Using the VEP plugin MaxEntScan, two SNPs were found to be associated with different entropies at splice sites. These SNPs were located in the serotonin transporter (SLC6A3) and the neuropeptide Y (NPY) genes and changed entropy from 0.40 to -4.66 and 9.38 to 9.43, respectively. These SNPs were identified as 'splice_region_variant' and 'intron_variant' by VEP and SnpEff. However, not all identified 'splice_region_variants' caused differences in splice site entropy, as predicted by MaxEntScan. An analysis of linkage disequilibrium using vcftools revealed that 64 SNPs were linked. Linkage r2 values ranged from 0.28 to $1(\varnothing 0.91)$. 
Table 2.2: General descriptive statisics of the detected SNPs in the investigated population of Assamese macaques

\begin{tabular}{|c|c|c|c|c|c|c|c|c|}
\hline \multirow[b]{2}{*}{ individuals } & \multicolumn{4}{|c|}{ in percentage } & \multicolumn{4}{|c|}{ in the number of individuals } \\
\hline & minimun & maximum & mean & $\begin{array}{l}\text { standard } \\
\text { deviation }\end{array}$ & minimun & maximum & mean & $\begin{array}{l}\text { standard } \\
\text { deviation }\end{array}$ \\
\hline $\begin{array}{l}\text { homozygous for } \\
\text { reference allele }\end{array}$ & 0.00 & 98.73 & 82.60 & 28.00 & 0.00 & 78.00 & 62.65 & 22.88 \\
\hline heterozygous & 0.00 & 56.96 & 10.28 & 15.29 & 0.00 & 45.00 & 7.83 & 11.82 \\
\hline $\begin{array}{l}\text { homozygous for } \\
\text { alternate allele }\end{array}$ & 0.00 & 100.00 & 7.12 & 20.10 & 0.00 & 78.00 & 4.28 & 11.43 \\
\hline
\end{tabular}

Table 2.3: Count and percent of consequence types of SNPs predicted by VEP and SnpEff

\begin{tabular}{lrrrr}
\hline \multirow{2}{*}{ Consequence type } & \multicolumn{2}{c}{ VEP } & \multicolumn{2}{c}{ SnpEff } \\
\cline { 2 - 5 } & Count & Percent & Count & Percent \\
\hline 3_prime_UTR_variant & 13 & $2.36 \%$ & 13 & $2.23 \%$ \\
5_prime_UTR_premature_ & 0 & $0 \%$ & 2 & $0.34 \%$ \\
start_codon_gain_variant & & & & \\
5_prime_UTR_variant & 6 & $1.09 \%$ & 6 & $1.03 \%$ \\
downstream_gene_variant & 20 & $3.62 \%$ & 20 & $3.44 \%$ \\
frameshift_variant & 0 & $0.00 \%$ & 0 & $0.00 \%$ \\
intergenic_variant & 11 & $1.99 \%$ & 39 & $6.70 \%$ \\
intron_variant & 260 & $47.10 \%$ & 260 & $44.67 \%$ \\
missense_variant & 62 & $11.23 \%$ & 62 & $10.65 \%$ \\
splice_region_variant & 19 & $3.44 \%$ & 19 & $3.26 \%$ \\
synonymous_variant & 105 & $19.02 \%$ & 105 & $18.04 \%$ \\
upstream_gene_variant & 56 & $10.14 \%$ & 56 & $9.62 \%$ \\
\hline total & 552 & $100.00 \%$ & 582 & $100.00 \%$ \\
\hline
\end{tabular}

Table 2.4: Count and percent of impact classes of SNPs predicted by VEP and SnpEff

\begin{tabular}{lrrrr}
\hline \multirow{2}{*}{ Consequence type } & \multicolumn{2}{c}{ VEP } & \multicolumn{2}{c}{ SnpEff } \\
\cline { 2 - 5 } & Count & Percent & Count & Percent \\
\hline moderate & 122 & $22.89 \%$ & 124 & $22.03 \%$ \\
low & 62 & $11.63 \%$ & 62 & $11.01 \%$ \\
modifier & 349 & $65.48 \%$ & 377 & $66.96 \%$ \\
\hline total & 533 & $100 \%$ & 563 & $100.00 \%$ \\
\hline
\end{tabular}




\section{Discussion}

Enhancing the approach of behavioural genetics and physiogenetics in wild animals would extend our knowledge of the factors that contribute to the still not completely understood variation within and between populations under natural selection pressures (Brent \& Melin, 2014). Behavioural studies often investigate the impacts of personality, age, sex or external factors such as social environment, group size and dominance hierarchy to explain inter-individual differences in HPA-axis related traits and probably misrepresent the amount of variation to be explained by such factors because they neglect genetic impacts (Baugh et al., 2017; Mell et al., 2016; Seyfarth et al., 2012; Young et al., 2014a) or focus only on one or two gene variants (Garamszegi et al., 2014; Kalbitzer et al., 2016; Timm et al., 2015; but see Bergey et al., 2016; Madlon-Kay et al., 2018; Song et al., 2017). An extended integration of genotype information in wild populations will facilitate a more comprehensive understanding of the observed phenotypic variation. For example, variant information on multiple loci can be used to generate cumulative genetic risk scores to predict individual variation (Belsky \& Israel, 2014; Ferguson et al., 2012). Among the best-studied aspects of animal behaviour in the wild are behavioural and physiological reactions to social and environmental stressors (Reeder \& Kramer, 2005). Behavioural and physiological responses are tightly linked to the HPA-axis, the main physiological pathway activated in response to stressful stimuli (Del Rey et al., 2008). Thus, genes coding for the components of the HPA-axis, which act in concert to maintain homeostatic balance, are important targets for the investigation of phenotypic variation in stress- and aggression-related traits.

We provide a SNP panel that may serve as a basic tool for future studies investigating the genetics of stress and aggression in behavioural and ecological studies. The offered panel and protocol enables field biologists teaming up with a laboratory to screen entire wild animal populations for multiple highly interesting target regions in a fast and parallel way. This study demonstrates that polymorphisms at purportedly functional sites exist in HPA-linked genes in natural populations. Knowing the samples from individually-recognized individuals, which is usually the case in long-term studies on wild animal groups, allows to accurately determine population frequencies of SNPs. Further, this application is transferable to other species. The HPA-axis is conserved 
among vertebrates (Denver, 2009; Schulkin, 2011) and orthologous gene regions can be found easily for the species of interest e.g. via BLAST (Altschul et al., 1990), PSIBLAST (Altschul et al., 1997), BLAT (Kent, 2002), SSEARCH (Smith \& Waterman, 1981, biology.wustl.edu/gcg/ssearch.html) or HMMER3 (hmmer.org) searches. For application to other species, we recommend searching for the primer or amplicon sequences (Supplementary Table S1.1) using the aforementioned software and choosing the respective sequences to design taxon-specific primers. All subsequent steps can be carried out as outlined in our protocol. With small PCR product sizes, as in our case, allelic dropout is a marginal problem, but to further minimize the risk of allelic dropout we recommend multiple PCRs per sample (Goodrich et al., 2014) or to perform replicates from different samples of the same individual.

Given that acquired massively parallel sequencing data hold many opportunities for further in-depth analyses, we provide detailed information on the applied bioinformatics pipelines. For example, with the help of GATK (McKenna et al., 2010) or SAMtools (Li et al., 2009) variants can be detected and used for subsequent highthroughput analyses concerning their functionality and possible effects (e.g. VEP, McLaren et al., 2016 and SnpEff, Cingolani et al., 2012). However, our analyses revealed that GATK called more variants than SAMtools in all conditions of the different quality thresholds and emphasize the importance of comparing pipelines and relying on validated, intersecting sets of SNPs. Further analyses of, e.g. splice site entropy (MaxEntScan) and linkage disequilibrium (vcftools), help to interpret the effects of detected polymorphisms and their potential consequences on physiological pathways.

To promote this application for studies on wild populations of non-model organisms, in which the consideration of genotype is particularly rare, we established our methods based on faecal samples. Studies on protected, free-ranging animals are often confined to the non-invasive collection of genetic material. DNA extracts from such lowquality sources contain only small amounts of host DNA (Perry et al., 2010). The dominance of exogeneous, non-host, e.g. microbiome or food DNA, rules out a massively parallel sequencing-application on the pure DNA extracts, without prior amplification or enrichment of target regions. Sequencing of amplicons with the traditional Sanger method is time- and cost-intensive, particularly when encountering larger sets of target regions and individuals (3066 amplicons in this study). Furthermore, when two SNPs are found in 
one amplicon, the haplotype structure remains unknown. When applying the classical Sanger sequencing approach, elucidating haplotypes requires additional working steps, such as cloning. Here we have shown that multiple target regions can be easily covered with massively parallel amplicon sequencing from faecal DNA of larger numbers of individuals. Alternatively, target regions could be captured using synthesized or selfmade capture probes (Maricic et al., 2010; Snyder-Mackler et al., 2016a). While such methods may reach better sequencing uniformity, they are less target-specific and exhibit lower average coverage than amplicon-based technologies (Samorodnitsky et al., 2015). However, such methods could be applied to calculate additional background population structure (Snyder-Mackler et al., 2016a). Another important aspect, especially for studies on wild populations that are often limited to low-quality DNA samples, is that ampliconbased massively parallel sequencing methods allow processing of low-input DNA samples (Samorodnitsky et al., 2015). Further, due to the large amount of sequence reads produced, the regions of interest (amplicons) exhibit high coverage reducing the detection of false positive variants. A caveat of the study is that it is still ultimately a bottom-up approach needing a priori information to select target regions. As technology will improve in the future, top-down approaches will most likely also become an effective and economical tool for low-quality samples making more data available. These top-down approaches could be applied to generate haplotype data for a multitude of loci across the genome in a fast and parallel way, to calculate relatedness and include kinship relations in behavioural genetics approaches as well as to identify conserved genome regions or gene segments with high mutation rates in the investigated populations.

Numerous field studies have established links between non-invasive measures of HPA-axis activity and the behaviour of animals. Glucocorticoid metabolite levels, the end products of the HPA-axis, increase during reproductive challenges (Ostner et al., 2008a, 2008b), with increasing aggression given or received (Ostner et al., 2008a; Wittig et al., 2015) and are often related to social status (Goymann et al., 2004). Mostly lab-based studies have established links between HPA-axis activity and genetic variation at individual loci. For example, a mu-opioid receptor polymorphism is associated with cortisol and aggressive threat scores (Miller et al., 2004) and variation in the serotonin transporter gene is associated with increased HPA-axis activity (Barr et al., 2004) in captive primates. Progress is hampered by a lack of 1) integration of both research streams to link genetic variation to HPA-axis activity and behaviour, and 2) studies 
screening multiple loci involved in HPA-axis regulation at the same time. We propose that our panel can serve as a basis for general behavioural studies aiming to extend their study design on a molecular level and step into the field of behavioural genetics. The simultaneous investigation of genes and behaviour will help to achieve a more comprehensive understanding of individual animal characteristics.

\section{Acknowledgements}

We thank the National Research Council of Thailand (NRCT) and the Department of National Parks, Wildlife and Plant Conservation (DNP) for permission to conduct this study and for all the support granted. We are grateful to J. Prabnasuk, K. Nitaya T. Wongsnak, M. Pongjantarasatien and K. Kreetiyutanont, M. Kumsuk, W. Saenphala (Phu Khieo Wildlife Sanctuary) for their cooperation and permission to carry out this study. We thank A. Koenig and C. Borries, who developed the field site at Huai Mai Sot Yai. Special thanks go to S. Jumrudwong, W. Nueorngshiyos, N. Juntuch, J. Wanart, R. Intalo, T. Kilawit, N. Pongangan, B. Klaewklar, N. Bualeng, A. Ebenau, P. Saisawatdikul, K. Srithorn, M. Swagemakers, and T. Wisate for their excellent help in the field. We acknowledge C. Schwarz, N. Westphal, F. Aron and L. Walter for their support in the genetics lab. We thank our colleagues, particularly F. Ludewig, from the Microarray and Deep-Sequencing Core Facility, University Medical Center Göttingen for sequencing. We also thank T. S. Gutleb for valuable comments on the manuscript. We thank the members of the research unit 'Sociality and Health in Primates' (DFG FOR 2136) for stimulating discussions. This research was funded by the Deutsche Forschungsgemeinschaft (DFG, German Research Foundation) - project number OS201/8-1; 254142454/GRK 2070. 


\section{Chapter 3}

\section{Study 2: COMT Val ${ }^{158}$ Met moderates the link between rank and aggression in a non-human primate}

Daria Raffaella Gutleb ${ }^{1,2,3}$, Christian Roos ${ }^{4,5}$, Angela Noll ${ }^{5}$, Julia Ostner ${ }^{1,2,3 \dagger}$, Oliver Schülke $\mathrm{s}^{1,2,3 \dagger}$

${ }^{1}$ Department of Behavioral Ecology, Johann-Friedrich-Blumenbach Institute for Zoology and Anthropology, University of Goettingen, Göttingen, Germany

${ }^{2}$ Research Group Social Evolution in Primates, German Primate Center, Leibniz Institute for Primate Research, Göttingen, Germany

${ }^{3}$ Leibniz ScienceCampus Primate Cognition, Göttingen, Germany

${ }^{4}$ Gene Bank of Primates, German Primate Center, Leibniz Institute for Primate Research, Göttingen, Germany

${ }^{5}$ Primate Genetics Laboratory, German Primate Center, Leibniz Institute for Primate Research, Göttingen, Germany

${ }^{\dagger}$ Contributed equally as senior authors

published in Genes, Brain and Behavior

2018; $17:$ e12443

doi: $10.1111 / \mathrm{gbb} .12443$

submitted 11.09.2017, revised 22.11.2017, accepted 28.11.2017 


\section{Abstract}

The COMT Val ${ }^{158}$ Met polymorphism is one of the most widely studied genetic polymorphisms in humans implicated in aggression and the moderation of stressful life event effects. We screened a wild primate population for polymorphisms at the COMT Val ${ }^{158}$ Met site and phenotyped them for aggression to test whether the human polymorphism exists and is associated with variation in aggressive behavior. Subjects were all adults from four study groups (37 males, 40 females) of Assamese macaques (Macaca assamensis) in their natural habitat (Phu Khieo Wildlife Sanctuary, Thailand). We collected focal animal behavioral data (27 males, 36 females, 5964 focal hours) and fecal samples for non-invasive DNA analysis. We identified the human COMT Val ${ }^{158} \mathrm{Met}$ polymorphism (14 Met/Met, $41 \mathrm{Val} / \mathrm{Met}, 22 \mathrm{Val} / \mathrm{Val})$. Preliminary results suggest that COMT genotype and dominance rank interact to influence aggression rates. Aggression rates increased with rank in Val/Val, but decreased in Met/Met and Val/Met individuals, with no significant main effect of COMT genotype on aggression. Further support for the interaction effect comes from time series analyses revealing that when changing from lower to hig^5her rank position $\mathrm{Val} / \mathrm{Val}$ individuals decreased, whereas Met/Met individuals increased their aggression rate. Contradicting the interpretation of earlier studies, we show that the widely studied $\mathrm{Val}{ }^{158}$ Met polymorphism in COMT is not unique to humans and yields similar behavioral phenotypes in a non-human primate. This study represents an important step towards understanding individual variation in aggression in a wild primate population and may inform human behavioral geneticists about the evolutionary roots of inter-individual variation in aggression. 


\section{Introduction}

Catechol-O-methyltransferase (COMT) is a key enzyme in the catecholamine catabolism (Männistö \& Kaakkola, 1999). It inactivates neurotransmitters in the brain, including the catecholamines dopamine, adrenaline and noradrenaline (Axelrod \& Tomchick, 1958). The COMT enzyme is encoded by the COMT gene, which is $27.22 \mathrm{~kb}$ in length and located on chromosome 22q11.2 in humans (Grossman et al., 1992). Since its characterization in 1958 (Axelrod \& Tomchick, 1958), COMT has been under extensive investigation and has become one of the most studied candidate genes (Dickinson \& Elvevåg, 2009), especially in human studies on stress and aggression (Qayyum et al., 2015).

COMT is expressed throughout the brain (Hong et al., 1998) and occupies a significant regulatory role particularly in the prefrontal cortex (PFC). COMT is not the only protein linked to dopamine clearance, as dopamine transporters remove dopamine from the synaptic cleft as well. However, due to the limited number of dopamine transporters present in the PFC, COMT accounts for more than $60 \%$ of dopamine degradation in this brain region (Käenmäki et al., 2010; Karoum et al., 1994; Matsumoto et al., 2003; Sesack et al., 1998; Yavich et al., 2007).

Variation in catecholamine levels have been associated with aggressive behavior (Coccaro et al., 1991; Puciłowski et al., 1986; Ratey \& Gordon, 1992). Dopamine levels increase shortly before, during and after aggressive interactions in rats (Tidey \& Miczek, 1996) and COMT-knockout mice exhibit increased levels of aggressive behavior (Gogos et al., 1998). Catecholamine agonists elevate aggressive behavior (Volavka, 2002) and specific dopaminergic antagonists are the most frequently applied pharmacotherapy in human aggression prevention (de Almeida et al., 2005; Gualtieri \& Schroeder, 1990; McDougle et al., 1998; Yudofsky et al., 1984). COMT has been established as a promising candidate gene for the regulation of neural processes leading to aggressive behavior (Qayyum et al., 2015; Volavka et al., 2004; but see Vassos et al., 2014).

Within the COMT gene, one functional polymorphism has been repeatedly associated with aggressive phenotypes in particular - the COMT $\mathrm{Val}^{158} \mathrm{Met}$ polymorphism (dbSNP: rs4680; Qayyum et al., 2015). Val ${ }^{158}$ Met is a non-synonymous 
single nucleotide polymorphism (SNP), located in exon 4 of the COMT gene. Due to a $\mathrm{G}$ to A transition, the amino acid valine (Val) is substituted by methionine (Met) at codon 158. This SNP influences the enzyme's catabolizing function of synaptic dopamine (Tunbridge, 2010; Witte \& Flöel, 2012). In contrast to the low-activity, thermolabile Met variant, the Val variant is considered to be the gene variant with higher enzymatic activity and heat-stability (Lotta et al., 1995). Carriers of the Met allele suffer from a $40 \%$ reduced enzyme activity at physiological body temperature of $37{ }^{\circ} \mathrm{C}$ (Chen et al., 2004). In turn, the Val genotype is associated with higher dopamine degradation and presumably lower dopamine levels in the PFC.

The effects of the COMT Val ${ }^{158}$ Met SNP have been studied extensively in conjunction with environmental variables. For example the influence of $\mathrm{Val}^{158} \mathrm{Met}$ on the severity of aggression in children (Hygen et al., 2015), antisocial behavior in attentiondeficit hyperactivity disorder (ADHD, Thapar et al., 2005), impulsive aggression in females (Wagner et al., 2010), adolescent aggressive behavioral problems (Brennan et al., 2011), behavioral and emotional problems in children (Thompson et al., 2012), depressive episodes (Hosang et al., 2017), and increased reactivity to stress as part of vulnerability for psychosis (Stefanis et al., 2007; van Winkel et al., 2008) is moderated by environmental risk factors such as prenatal stress, severe and stressful life events as well as by environmental protective factors such as parental warmth.

It should be noted that studies on the influence of the COMT $\mathrm{Val}^{158} \mathrm{Met}$ polymorphism on human aggression have mostly used various indirect measures of aggression. Common methods are aggression score questionnaires, such as the Overt Aggression Scale (OAS, Yudofsky et al., 1986, e.g. Han et al., 2004), the Risk Assessment for Dangerousness (RAD, e.g. Strous et al., 1997), or the Buss-Durkee Hostility Inventory (BDHI, Buss \& Durkee, 1957, e.g. Wagner et al., 2010). Difficulties with comparing and interpreting these results as well as in defining and measuring aggression have been pointed out (Qayyum et al., 2015). Issues in human studies arise from not differentiating between antisocial and aggressive behavior, questionnaire heterogeneity and the use of self-reported ratings instead of data on the actual expression of aggressive behavior (Qayyum et al., 2015).

In behavioral studies on non-human primates, aggression can be quantified reliably via direct observation of aggressive interactions. The calculation of aggression 
rates enables a direct quantitative comparison between individuals and species. Aggression is an integral part of primate social behavior (de Waal, 1992). Dyadic and polyadic aggression serves to attain and maintain high social status (Chapais, 1995; Schülke et al., 2010) and social status in turn affects health (Habig \& Archie, 2015; Sapolsky, 2005; Snyder-Mackler et al., 2016b) and Darwinian fitness (Majolo et al., 2012). As a consequence, aggression is typically directed down the dominance hierarchy (Ostner et al., 2008a; Rowell, 1971; Schino et al., 2005; Silk, 1982) and dominance also affects patterning of affiliative behavior among group members (Schino, 2001; Seyfarth, 1977; Silk et al., 2006a, 2006b). Thus, any analysis of the drivers of individual aggressive behavior in primates needs to consider that dominance rank and aggression may be tightly linked.

While COMT Val ${ }^{158}$ Met has been studied extensively in humans, it is currently unknown whether this polymorphism exists in non-human primates and whether or not it is associated with similar behavioral phenotypes. In this study, we aimed at extending previous findings on the role of the COMT gene and aggression by (1) identifying existing COMT polymorphisms at the $\mathrm{Val}^{158} \mathrm{Met}$ site in a non-human primate, and (2) assessing the effect of genotype on aggressive behavior using direct measures of aggression rates. As the effects of COMT $\mathrm{Val}^{158} \mathrm{Met}$ have been described as moderating and aggression and dominance rank are interconnected in non-human primate societies, we assessed a possible moderating effect of the polymorphism on the relationship between dominance rank and rates of overt aggression. We studied wild Assamese macaques (Macaca assamensis) living in large multimale-multifemale groups and expressing a strictly linear steep dominance hierarchy (Bernstein \& Cooper, 1999; Macdonald et al., 2013; Ostner et al., 2008a, 2011). Aggressive social interactions are ubiquitous and aggression frequencies peak during the mating season (Ostner et al., 2008a, 2011). 


\section{Materials and methods}

\section{Ethical statement}

Behavioral data and fecal samples were collected non-invasively. Our research adhered to the protocols approved by the Animal Welfare Body of the German Primate Center (Deutsches Primatenzentrum, DPZ) in Germany and the American Society of Primatologists (ASP) Principles for the ethical treatment of non-human primates (https://www.asp.org/society/resolutions/EthicalTreatmentOfNonHumanPrimates.cfm). The National Research Council of Thailand (NRCT) and the Department of National Parks, Wildlife and Plant Conservation (DNP) authorized (permit numbers: 0004.3/3618, $0002.3 / 2647,0002 / 17,0002 / 626,0002 / 2424)$ the data collection at the study site in Thailand and the export of samples based on a benefit sharing agreement.

\section{Study site and population}

This study was conducted at Phu Khieo Wildlife Sanctuary (PKWS; $16^{\circ} 5^{\prime}-35^{\circ} \mathrm{N}$, $101^{\circ} 20^{\prime}-55^{\circ} \mathrm{E}, 300-1300 \mathrm{~m}$ above sea level) in the province of Chaiyaphum, north-eastern Thailand (Schülke et al., 2011). The sanctuary is part of the $6500 \mathrm{~km}^{2}$ protected Western Isaan Forest Complex, comprising eight protected areas (Grassman et al., 2005). The study area includes dry and hill evergreen forest, dry dipterocarp forest, bamboo patches (Borries et al., 2002) and is inhabited by a diverse community of large mammals and predators suggesting low levels of habitat disturbance.

Our study population consisted of four fully habituated wild groups of Assamese macaques ranging within their natural habitat in PKWS. The macaques have been observed in a long-term field project since 2005 and all individuals were identified reliably. Here, we focused on the adult individuals of the groups. Males are considered adult when they have fully developed testes, adult male body length, long canines, are involved in reproduction and outrank all adult females (Ostner et al., 2008a). Females 
were classified as adult from the beginning of the mating season they had first conceived in, based on the observation of their first birth.

\section{COMT genotyping}

We collected fecal samples from all individuals that were adult at the beginning of March 2015 (27 males, 36 females). For the analysis of genotype frequency, we included samples collected since 2006 from adult males $(n=10)$ and females $(n=4)$ that had died or emigrated. About $5 \mathrm{~g}$ of feces were removed immediately after defecation from the surface of the sample from an identified individual and transferred into $50 \mathrm{ml}$ falcon tubes (62.559.001, Sarstedt, Nümbrecht, North Rhine-Westphalia, Germany) containing $30 \mathrm{ml}$ of $97 \%$ ethanol (Nsubuga et al., 2004). Samples were handled using disposable gloves and spoons. We applied the two-step storage procedure, which included the storage of fecal samples in alcohol for 24 to 36 hours (Nsubuga et al., 2004). Ethanol was then poured off and samples transferred into $50 \mathrm{ml}$ falcon tubes containing silica (112926-002, Intereducation Supplies Co., Ltd., Bangkok, Thailand, Roeder et al., 2004). Samples were stored at room temperature in the dark until DNA extraction was performed. DNA extraction was carried out with the First-DNA all-tissue Kit (D1002000, GEN-IAL $\mathrm{GmbH}$, Troisdorf, North Rhine-Westphalia, Germany), following the manufacturers protocol for DNA extraction from feces. DNA concentration was measured with NanoDrop (Spectrophotometer ND-1000, PEQLAB Biotechnologie GmbH, Erlangen, Bavaria, Germany) and extracts diluted to a concentration of $100 \mathrm{ng} / \mu \mathrm{l}$.

The target region was amplified with $1 \mathrm{U}$ BioThermTaq DNA Polymerase (GC002-5000, Genecraft, Cologne, North Rhine-Westphalia, Germany) in a $30 \mu \mathrm{l}$ PCR mix (1 $\mathrm{x}$ reaction buffer, $0.16 \mathrm{mM}$ for each $\mathrm{dNTP}, 0.33 \mu \mathrm{M}$ for each primer, and $18 \mathrm{ng} \mathrm{BSA}, 100$ ng DNA). PCR procedure was $94^{\circ} \mathrm{C}$ for 2 minutes, $60 \mathrm{x}\left(94^{\circ} \mathrm{C}\right.$ for 30 seconds, $54^{\circ} \mathrm{C}$ for 30 seconds, $72^{\circ} \mathrm{C}$ for 30 seconds; Labcycler, Sensoquest, Göttingen, Lower Saxony, Germany) including the following oligonucleotide primers: forward: 5' TACTCAGCTGTGCGCATG-3', reverse: 5'-AATGAACGTGGTGTGAACC-3'. As genome data were not available for Assamese macaques, we designed primers according to the rhesus macaque (Macaca mulatta) genome sequence. Sanger sequencing (ABI 
3130xl genetic analyzer, Big Dye Cycle Sequencing Kit, Thermo Fisher Scientific, Waltham, Massachusetts, USA) was applied to two samples to prove primer-specificity. All amplicons were submitted to Illumina next-generation sequencing (NGS; MiSeq paired-end $150 \mathrm{bp}$ ). The laboratory protocol included the preparation of sequencing libraries following the methods described in Rohland et al. (2015) without uracil-DNAglycosylase treatment based on Meyer \& Kircher (2010) and Kircher et al. (2012).

\section{Behavioral observations}

Assamese macaques are seasonal breeders, with a distinct non-mating season from March to September and mating season from October to February (Fürtbauer et al., 2010). All individuals that had reached the adult age at the beginning of this study in March 2015 were included in behavioral observations. We collected data from all adult males $(n=27)$ from March 1, 2015 to February 29, 2016 and all adult females $(n=36)$ from March 1, 2015 to February 28, 2017, thus including two entire non-mating and the consecutive mating seasons.

The study groups were followed from dawn to dusk and from sleep tree to sleep tree. During 40 minutes focal animal observations all agonistic, affiliative and sexual interactions were recorded continuously (Martin \& Bateson, 1993) to measure individual rates of interaction. In addition, we collected ad libitum data on agonistic interactions for the establishment of the dominance hierarchy. An effort was made to balance focal animal protocols across individuals and time of day (mean \pm SD $98 \pm 24$ hours/male, $95 \pm$ 27 hours/female, 5964 hours total). Between the non-mating and the mating season 2015 (September 11 to October 20) five adult males changed groups. Therefore, this time period was excluded from the male behavioral data analyses outlined below. 


\section{Data analyses}

\section{Genetic analyses}

The acquired Illumina reads were quality filtered using Trimmomatic v0.3668 and mapped (BWA MEM v0.7.1269) against the genome of Macaca mulatta v8. The polymorphism was identified by two different variant calling approaches (GATK, McKenna et al., 2010, and SAMtools, Li et al., 2009). Protein structure analysis was carried out with the freely available online tool PCI-Based Protein Secondary Structure Site Prediction Server (PCI-SS, http://bioinf.sce.carleton.ca/PCISS/start.php, Green et al., 2009; Green \& Korenberg, 2006) using the protein sequence of Homo sapiens (Accession Number: CAG30308.1) and Macaca mulatta (Accession number: NP_001247941.1). As genomic data were not available for our study species, for comparative analyses on both the DNA and the protein level, information available for the closely related rhesus macaque was used.

\section{Aggression rate}

From focal animal protocols we calculated an hourly aggression rate for each animal during the non-mating and the mating season. For females, data from two nonmating and two mating seasons were combined for analysis. In our study population the aggression rate, measured as being involved in a dyadic conflict and expressing aggression (i.e. open-mouth, growl, stare, headbob, point and repeated growl/yell) per hour, increases from the non-mating to the mating season (see Figure S2.1 and Ostner et al., 2008a). For females, we subtracted the aggression directed towards the infants of the respective year from their total aggression, because increased restraint of the infants by mothers might be misinterpreted as increased aggression (Berghänel et al., 2016). 


\section{Hierarchy}

To calculate the dominance hierarchy, we used all decided dyadic agonistic interactions from the continuous and the ad libitum data, where clear submissive behavior (i.e. silent-bared teeth display, make room and unprovoked give ground) was observed (e.g. Haunhorst et al., 2017). We calculated separate hierarchies for females and males, and for males during the non-mating and the mating season (due to males changing group) with the package DomiCalc (Schmid \& de Vries, 2013). We used 'Combi1' ordinal ranks, which represent a hierarchical combination of two DomiCalc indices (PD and Dom-Sub) and then standardized rank within groups to scale between -1 (lowest ranking) and 1 (highest ranking) to allow for comparisons between differently sized groups.

\section{Aggression and rank change}

During our study period 22 males changed their rank positions between the nonmating and the mating season. To assess within-individual effects of the genotypedominance rank interaction on aggression rate, we subtracted the aggression rate during the period of being lower-ranking from that during the period of being higher-ranking for each individual.

\section{Statistics}

Statistical analyses were conducted using R 3.1.2 (The R Foundation for Statistical Computing, Vienna, Austria, http://www.r-project.org). To investigate the impact of COMT genotype and rank on aggression, we ran a general linear model (aggression rate-model). To achieve normal distribution of the response aggression rate, it was square-root transformed. The aggression rate-model contained a two-way interaction 
between COMT genotype and rank as predictors. We included sex and season as control variables, because males generally express higher levels of aggression and aggression increases during the mating season in this and other macaque species (e.g. Berghänel et al., 2011; Eaton et al., 1981; Wilson \& Boelkins, 1970). Group ID was included as a control factor and animal ID as a random factor. For this model, we excluded four males from whom very little behavioral data were available: two males who died due to predation in May 2015 and two males who only stayed in the very periphery of the group for approximately one month.

To investigate how aggression changes with rank in different COMT genotypes, we ran a multiple linear regression model (rank change-model) with the change in aggression rate (rate at high minus rate at low rank) as a response variable. The rank change-model included the seasonal change (non-mating to mating season and vice versa) as a control variable, because aggression is generally higher during the mating season (see above). COMT genotype was included as a predictor and group ID as a control factor.

Both models were calculated with the package 'ImerTest' (Bates et al., 2015; Kuznetsova et al., 2017) with alpha levels set to 0.05. Various model diagnostics were applied to confirm model validity: visual inspection of distribution of residuals, qqplots, residuals plotted against fitted values, assessing aggression rate-model stability using the function 'glmm stability' written by Roger Mundry (MPI Evolutionary Anthropology, Leipzig, Germany), assessing leverage and dfbetas for single samples and levels of the random factor animal ID using the package 'influence.ME' (Niewenhuis et al., 2013), and variance inflation factors for the aggression rate-model using the package 'car' (Fox \& Weisberg, 2011). None of the diagnostics suggested a violation of the model assumptions. A comparison of the full models to the null models (excluding rank and COMT genotype in the aggression rate-model and COMT genotype in the rank change-model) using a likelihood ratio test with the $\mathrm{R}$ function 'anova', setting the argument to 'Chisq', revealed a trend in significance (aggression rate-model $\mathrm{p}=0.082$, rank change-model $\mathrm{p}=0.084$ ). This might be the case, because the control variables sex and season included in the null model have a very high impact on aggression rates. 


\section{Results}

\section{COMT genotype}

We found the adult population of Assamese macaques at PKWS to exhibit the same COMT $\mathrm{Val}^{158} \mathrm{Met}$ polymorphism that is found in humans. DNA sequences were submitted to the NCBI GenBank with the accession numbers MF356536 and MF356537 as well as to the Sequence Read Archive (SRA, SRP116685). In our sample of 77 adults that resided in the study groups since 2005 the genotype frequency was $18.2 \%$ homozygous Met/Met, $53.2 \%$ heterozygous Val/Met and $28.6 \%$ homozygous Val/Val (Table 3.1).

Protein structure was analyzed with PCI-SS using the protein sequence of Homo sapiens and Macaca mulatta. Structures of human and rhesus macaque COMT are similar. The human COMT protein is encoded by 271 amino acids and the COMT Val ${ }^{158}$ Met polymorphism is, as the name depicts, located at codon 158 . The rhesus macaque COMT protein is encoded by 270 amino acids and the identified COMT polymorphism in the homologous region is located at codon 157 . Thus, we refer to the polymorphism detected in our macaque species, as the COMT Val ${ }^{157}$ Met polymorphism. Both in humans and macaques the polymorphism lies within the random coil structure of the protein.

Table 3.1: Genotype frequency of the COMT $\mathrm{Val}^{157} \mathrm{Met}$ polymorphism in wild Assamese macaques at PKWS

\begin{tabular}{lrrrrrr}
\hline \multicolumn{1}{c}{ Genotype } & \multicolumn{2}{c}{ All } & \multicolumn{2}{c}{ Males } & \multicolumn{2}{c}{ Females } \\
\hline & $\mathrm{n}$ & $\%$ & $\mathrm{n}$ & $\%$ & $\mathrm{n}$ & $\%$ \\
\cline { 2 - 8 } Met/Met & 14 & 18.2 & 7 & 18.9 & 7 & 17.5 \\
Val/Met & 41 & 53.2 & 21 & 56.8 & 20 & 50.0 \\
Val/Val & 22 & 28.6 & 9 & 24.3 & 13 & 32.5 \\
\hline Total & 77 & 100 & 37 & 100 & 40 & 100 \\
\hline
\end{tabular}




\section{Aggression rate}

The mean aggression rate of males was $0.30 \pm 0.14$ acts per hour in the nonmating season and $0.55 \pm 0.22$ in the mating season (Wilcoxon signed-rank test: $n=22$, $\mathrm{p}<0.001, \mathrm{z}=-3.588$; Figure S2.1). The mean aggression rate of females was much lower at $0.08 \pm 0.06$ acts per hour in the non-mating season and $0.09 \pm 0.07$ in the mating season (Wilcoxon signed-rank test: $\mathrm{n}=36, \mathrm{p}=0.212, \mathrm{z}=-0.803$ ).

The aggression rate-model revealed a significant influence of sex, with males having higher aggression levels than females $(\mathrm{t}=13.15, \mathrm{p}<0.001)$, and a significant negative influence of the non-mating season compared to the mating season $(\mathrm{t}=-3.19$, $\mathrm{p}=0.002$ ) on aggression rate (Table 3.2). The interaction term of COMT genotype and rank significantly influenced aggression rates (Met/Met-Val/Val: $\mathrm{t}=-2.02, \mathrm{p}=0.046$, Val/Met-Val/Val: $\mathrm{t}=-2.046, \mathrm{p}=0.043$, Table 3.2 and 3.3). Plotting the partial residuals of the model revealed that aggression rates increased with dominance rank in $\mathrm{Val} / \mathrm{Val}$ individuals, but decreased in Met/Met and Val/Met (Figure 3.1). This result is supported by running the same model, but exchanging the predictor of 'COMT genotype' with the dichotomous variable 'COMT carrier Met' $\left(\mathrm{R}^{2}=0.665\right.$, comparison to null model $\mathrm{p}=0.026$, see Table S2.1 and Figure S2.2). Individuals carrying at least one Met-allele increased their aggression rate with increasing rank, whereas non-Met-carriers (= homozygous $\mathrm{Val} / \mathrm{Val})$ decreased their aggression rate with increasing rank $(\mathrm{t}=2.397, \mathrm{p}=0.018$, for this model please see Table S2.1 and Figure S2.2). The aggression rate-model explained $66 \%$ of the variation in aggression rates.

Table 3.2: Aggression rate-model with COMT genotype Met/Met set as reference

\begin{tabular}{lrrrr}
\hline Fixed effect & Estimate & Std. Error & t-value & p-value \\
\hline Intercept & 0.347 & 0.042 & 8.259 & $<0.001$ \\
COMT genotype Met/Val & -0.017 & 0.036 & -0.478 & 0.633 \\
COMT genotype Val/Val & -0.005 & 0.039 & -0.127 & 0.899 \\
Rank & 0.082 & 0.046 & 1.786 & 0.077 \\
Sex: male & 0.353 & 0.027 & 13.147 & $<0.001$ \\
Season: non-mating & -0.080 & 0.025 & -3.190 & 0.002 \\
COMT genotype Met/Val*rank & 0.026 & 0.055 & -0.471 & 0.638 \\
COMT genotype Val/Val*rank & -0.117 & 0.058 & -2.017 & 0.046 \\
\hline n=117 data points for 59 individuals (23 males and 36 females) and two seasons &
\end{tabular}

$\mathrm{n}=117$ data points for 59 individuals ( 23 males and 36 females) and two seasons 


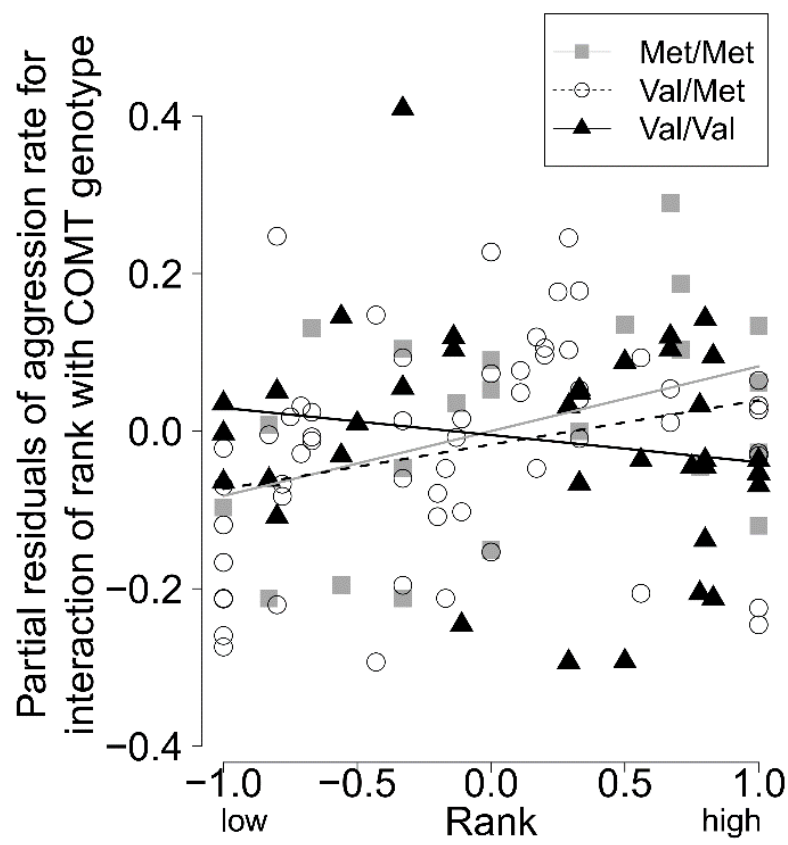

Figure 3.1: Partial residuals of the response variable aggression rate per hour for the interaction term of COMT genotype and rank in the aggression rate-model.

Table 3.3: Aggression rate-model releveled with COMT genotype Val/Met set as reference

\begin{tabular}{lrrrr}
\hline Fixed effect & Estimate & Std. Error & t-value & p-value \\
\hline Intercept & 0.330 & 0.031 & 10.635 & $<0.001$ \\
COMT genotype Met/Met & 0.017 & 0.036 & 0.478 & 0.633 \\
COMT genotype Val/Val & 0.012 & 0.029 & 0.412 & 0.681 \\
Rank & 0.056 & 0.028 & 1.999 & 0.048 \\
Sex: male & 0.353 & 0.027 & 13.147 & $<0.001$ \\
Season: non-mating & -0.080 & 0.025 & -3.190 & 0.002 \\
COMT genotype Met/Met*rank & 0.026 & 0.055 & 0.471 & 0.638 \\
COMT genotype Val/Val*rank & -0.091 & 0.044 & -2.046 & 0.043 \\
\hline
\end{tabular}

$\mathrm{n}=117$ data points for 59 individuals ( 23 males and 36 females) and two seasons

\section{Aggression and rank change}

The mating season had a strong influence on changes in aggression rate in males ( $\mathrm{t}=4.065, \mathrm{p}=0.001$, Table 3.4). The model revealed that individuals with the COMT genotype $\mathrm{Val} / \mathrm{Val}$ exhibited decreased aggression rates when changing from a lower to a higher rank position compared to Met/Met individuals (Met/Met-Val/Val: $t=-2.204$, p=0.044, Table 3.4 and Figure 3.2) when controlling for the seasonal effect. On average, 
Val/Val individuals decreased their aggression rate by $-0.15(-1.88 \%)$, whereas Met/Met individuals increased their aggression rate by $0.08(64.74 \%)$ when changing from a lower to a higher rank position. No significant difference between the heterozygous Val/Met individuals and Val/Val and Met/Met individuals was observed (Val/Met-Met/Met: $\mathrm{t}=1.554, \mathrm{p}=0.141, \mathrm{Val} / \mathrm{Met}-\mathrm{Val} / \mathrm{Val}: \mathrm{t}=-0.883, \mathrm{p}=0.391$, see Table $\mathrm{S} 2.3)$. A comparison between individuals of Met/Met and $\mathrm{Val} / \mathrm{Val}$ genotypes revealed that the expected value of the response variable 'change in aggression rate per hour' varies by -0.38 when keeping all other control variables constant. The multiple $\mathrm{R}^{2}$ of the rank change-model was 0.611.

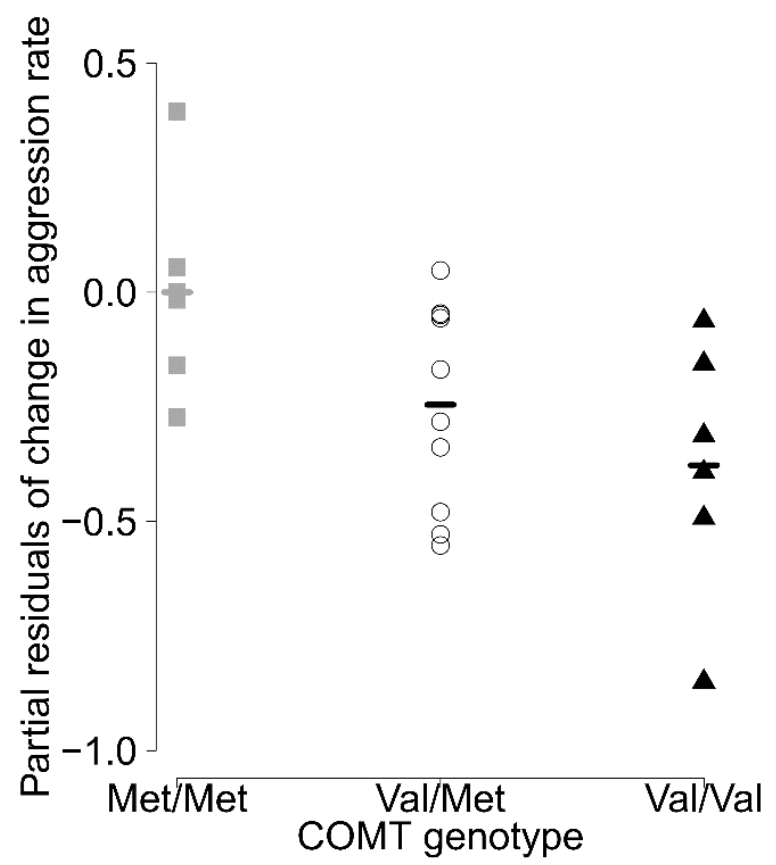

Figure 3.2: Partial residuals of change in aggression rate associated with an increase in dominance rank compared between COMT genotypes in the rank change-model. Coefficients are depicted as a horizontal line for each genotype with Met/Met being the reference.

Table 3.4: Rank change-model of aggression (within individuals changing from a lower to a higher rank position) with COMT genotype Met/Met set as reference

\begin{tabular}{lrrrr}
\hline Effect & Estimate & Std. Error & t-value & p-value \\
\hline Intercept & -0.240 & 0.139 & -1.725 & 0.105 \\
COMT genotype Met/Val & -0.246 & 0.158 & -1.554 & 0.141 \\
COMT genotype Val/Val & -0.378 & 0.171 & -2.204 & 0.044 \\
Season change: non-mating to mating & 0.496 & 0.122 & 4.065 & 0.001 \\
\hline $\mathrm{n}=22$ males & & & &
\end{tabular}




\section{Discussion}

The COMT $\mathrm{Val}^{158} \mathrm{Met}$ polymorphism is one of the most studied functional candidate polymorphisms in human behavioral genetics. We identified this SNP, which had not been known to exist in other species besides humans, in a wild macaque population. Studies on the human COMT Val ${ }^{158} \mathrm{Met}$ polymorphism cite Palmatier et al. (1999) for showing that the COMT $\mathrm{Val}^{158}$ Met polymorphism does not exist in non-human primates and that the high activity Val-allele, and not the low activity Met-allele, represents the ancestral state. Provided that the allelic frequency of COMT Val ${ }^{158} \mathrm{Met}$ has a wide range (0.01 to 0.62$)$ in human populations, sample sizes for non-human primates (1-2 individuals of 4 species in Palmatier et al., 1999) may have been too low to warrant these conclusions. From publically available DNA sequences 85 today it is known that both the Val and the Met allele occur in one or the other macaque species (M. mulatta, M. fuscata: Met, M. fascicularis, M. nemestrina: Val, Pflüger et al., 2016). This study is the first to identify this polymorphism in a non-human primate species and to link the genotypic variation to behavior.

Our results show that the interaction of the COMT $\mathrm{Val}^{157} \mathrm{Met}$ polymorphism and dominance rank is associated with aggression in Assamese macaques. Most human studies revealed Met-individuals as the 'worrier' type, compared to the 'warrior' type of Val-individuals (Goldman et al., 2005; Stein et al., 2006). The warrior-worrier model is an explanation for the maintenance of both COMT alleles due to counterbalancing effects in stress resilience, anxiety and cognition (Goldman et al., 2005). The Met-allele represents the low activity variant of the COMT protein (Chen et al., 2004; Lotta et al., 1995) which presumably leads to a reduced clearance of catecholamines, such as dopamine, in the brain. Increased dopamine levels are generally associated with increased levels of aggression (e.g. Tidey \& Miczek, 1996).

We did not find a direct link between the COMT genotype and aggression. Rather, aggression rates were predicted by an interaction of COMT genotype and a salient social condition in these primates - their dominance rank position. Met/Met- and Val/Metindividuals exhibited a positive relationship between aggression rate and dominance rank. The higher-ranking individuals were, the more aggression they expressed. Val/Val- 
individuals had an inverse relationship between rank and aggression rates; the higherranking $\mathrm{Val} / \mathrm{Val}$ individuals were, the less aggression they expressed. After we detected this overall genotype-dominance rank interaction effect in our first model, we investigated how individuals with different genotypes altered their aggression rate when changing dominance rank position in a within-individual analysis. In the subset of individuals that changed their dominance rank during our study period, Val/Valindividuals indeed had lower aggression rates in a higher compared to a lower rank position. We conclude that the COMT $\mathrm{Val}^{157}$ Met polymorphism moderates the link between dominance rank and aggression. Future studies are needed to replicate these results with much larger sample sizes in which small effects of single loci can be detected with higher certainty. The size of the interaction effect on aggression rate is small compared to the main effects of sex and mating season. This is important regarding the effects of single loci on behavior, compared to other environmental and sex effects. The simultaneous assessment of multiple loci might explain a larger proportion of the observed phenotypic variation.

In macaque societies dominance rank plays a pivotal role in everyday life and affects how individuals interact with their social environment (e.g. Sueur \& Petit, 2008; Thierry, 2000; Varley \& Symmes, 1966). Dominance rank may restrict or open up certain possibilities for how to interact with others and thus represents a salient social condition for macaques. The dominance hierarchy of Assamese macaque is steep and linear (Bernstein \& Cooper, 1999; Macdonald et al., 2013; Ostner et al., 2008a, 2011) and rank is acquired via aggressive coalitions in males (Schülke et al., 2010) and matrilinear support in females. Higher-ranking individuals occupy more central positions within the group and have privileged access to food resources and mating partners (e.g. Heesen et al., 2014, 2015; Ostner et al., 2011; Sukmak et al. 2014a). Levels of counter-aggression are low (Macdonald et al., 2013) and overall, aggression is directed down the hierarchy (Ostner et al. 2008a). The observation that depending on genotype some low ranking adults express higher aggression rates than some higher ranking ones may be explained by the redirection of aggression to immatures.

That aggression rates vary with dominance rank dependent on the genotype is suggestive of an underlying gene-environment interaction, with different social environments causing different behavioral outcomes in individuals with differing 
genotypes. In studies with humans, genotypic effects on aggression have also been investigated as moderating factors of life circumstances (e.g. Conway et al., 2012; Gallardo-Pujol et al., 2013; Reif et al., 2007; Simons et al., 2011; Wagner et al., 2010). It has been previously suggested that the relationship between COMT $\mathrm{Val}^{158} \mathrm{Met}$ and aggression may not be straightforward and the consideration of gene-environment interactions could help to elucidate variation in aggression (Qayyum et al., 2015). Particularly, the interplay of social environment, genes, and aggression in humans has been emphasized with regard to the 'differential susceptibility perspective' (Belsky, 1997; Belsky et al., 2007; Belsky \& Pluess, 2009a, 2009b; Simons et al., 2011). In support of this perspective the SNP moderates the effect between serious life events and aggression as well as parenting and alcohol use, with slopes of the different genotypes crossing over (Hygen et al., 2015; Laucht et al., 2012). Similarly, the regression lines crossover in our results on non-human primates, with the slopes reflecting contrastive effects. Both COMT alleles might act as a risk factor or advantage in different environmental conditions (Goldman et al., 2005; Weeland et al., 2015).

Additional evidence for the functional similarity of COMT in humans and nonhuman primates comes from a study targeting the region surrounding the COMT $\mathrm{Val}^{158} \mathrm{Met}$ polymorphism in a population of Japanese macaques (Macaca fuscata). These macaques do not carry the candidate SNP, but a polymorphic putative splice site forming three haplotypes (HT1, HT2, HT3), which are associated with physiological stress levels during the mating season (Pflüger et al., 2016). Similar associations between COMT variants and physiological stress have been found in humans (Armbruster et al., 2012; Bouma et al., 2012; Jabbi et al., 2007; Walder et al., 2010). Pflüger and colleagues (2016) found no association between different COMT haplotypes and aggression in Japanese macaques. Behavioral measures of aggression differ between the two studies, as in the Pflüger et al. (2016) study aggression data were collected ad libitum, were restricted to interactions among adult males during the mating season, and were expressed as the proportion of initiated aggression relative to other male-male behavior. Thus, it remains to be tested to what extent COMT $\mathrm{Val}^{157} \mathrm{Met}$ and the other three detected haplotypes lead to similar effects in non-human primates.

Among others, the COMT gene is conserved in humans, non-human primates, dogs, cows, rats, chicken and frogs (HomoloGene: 30982, NCBI Resource Coordinators, 
2017). The mechanism leading to a $40 \%$ reduced enzyme activity of the COMT Metvariant is located on the surface of the COMT protein (Vidgren et al., 1994; Zubieta et al., 2001). Enzyme stability is higher if the protein surface is more hydrophobic (Liu \& Wang, 2003; Machius et al., 2003). Thus, it has been proposed that due to the higher hydrophoby of Val compared to Met, it is more stable (Chen et al., 2004). Due to the high conservation of COMT between macaques and humans, it is very likely that the same mechanism applies also in this species. Future studies have to validate this functionality, because our study on a wild population in a protected area had to be strictly non-invasive.

Genetic factors explain up to $50 \%$ of variation in aggressive behavior in humans (Brendgen et al., 2006; Miles \& Carey, 1997; Rhee \& Waldman, 2002). COMT is one of the most studied candidate genes and has been extensively studied regarding aggression, yet other genes are also involved in aggressive behavior, e.g. monoamine oxidase A $(M A O A)$, which is involved in catecholamine degradation, and serotonin pathway genes (e.g. Bouma et al., 2012; Buckholtz \& Meyer-Lindenberg, 2008; Cases et al., 1995; Holmes et al., 2002; Lesch \& Merschdorf, 2000; Zammit et al., 2004). Future studies might also consider the simultaneous investigation of more than one candidate gene. This approach combined with an increased consideration of wild animal populations for the investigation of behavioral genetics will help to further elucidate mechanisms determining individual phenotypic variation. A crucial step is to include natural behavior measurements as well as environmental mediators to understand the implications of gene variants involved in aggression for individuals living in social groups.

\section{Acknowledgements}

We thank the National Research Council of Thailand (NRCT) and the Department of National Parks, Wildlife and Plant Conservation (DNP) for permission to conduct this study and for all the support granted. We are grateful to J. Prabnasuk, K. Nitaya T. Wongsnak, M. Pongjantarasatien and K. Kreetiyutanont, M. Kumsuk, W. Saenphala (PKWS) for their cooperation over the years and permission to carry out this study. We thank A. Koenig and C. Borries, who developed the field site. Special thanks go to $\mathrm{S}$. Jumrudwong, W. Nueorngshiyos, N. Juntuch, J. Wanart, R. Intalo, T. Kilawit, N. 
Pongangan, B. Klaewklar, N. Bualeng, A. Ebenau, P. Saisawatdikul, K. Srithorn, M. Swagemakers and T. Wisate for their excellent help in the field. We acknowledge Christiane Schwarz, Nico Westphal and Franziska Aaron for their support in the genetics lab. We also thank Holger Sennhenn-Reulen and Nadine Müller for statistical advice and Timon Salar Gutleb for helpful comments on the manuscript. We thank the members of the research unit 'Sociality and Health in Primates' (DFG FOR 2136) for stimulating discussions. This research was supported by the German Research Foundation (DFG) as part of the grant number OS201/8-1 and the RTG 2070 Understanding Social Relationships. The authors declare no conflict of interest. 


\section{Chapter 4}

\section{Study 3: A multi-locus genetic risk score modulates social buffering of HPA axis activity in wild male primates}

Daria Raffaella Gutleb ${ }^{1,2,3}$, Christian Roos ${ }^{4,5}$, Michael Heistermann ${ }^{6}$, Angela Noll ${ }^{5}$, Oliver Schülke ${ }^{1,2,3^{*}}$, Julia Ostner ${ }^{1,2,3 *}$

${ }^{1}$ Department of Behavioral Ecology, Johann-Friedrich-Blumenbach Institute for Zoology and Anthropology, University of Goettingen, Kellnerweg 6, 37077 Göttingen, Germany

${ }^{2}$ Research Group Social Evolution in Primates, German Primate Center, Leibniz Institute for Primate Research, Kellnerweg 4, 37077 Göttingen, Germany

${ }^{3}$ Leibniz ScienceCampus Primate Cognition, Kellnerweg 4, 37077 Göttingen, Germany

${ }^{4}$ Gene Bank of Primates, German Primate Center, Leibniz Institute for Primate Research, Kellnerweg 4, 37077 Göttingen, Germany

5 Primate Genetics Laboratory, German Primate Center, Leibniz Institute for Primate Research, Kellnerweg 4, 37077 Göttingen, Germany

${ }^{6}$ Endocrinology Laboratory, German Primate Center, Leibniz Institute for Primate Research, Kellnerweg 4, 37077 Göttingen, Germany

* Contributed equally as senior authors

manuscript in preparation 


\section{Abstract}

Different aspects of the social environment are associated with variation in health and fitness in animals and humans. Integrating adverse and beneficial effects of sociality, the social buffering hypothesis proposes that strong affiliative social bonds ameliorate the negative effects of environmental disturbances like overt conspecific aggression by attenuating the activity of the hypothalamic-pituitary-adrenal (HPA) axis. Social buffering effects are widespread across animals but the sources of within species variation in efficacy remain understudied. We assessed the modulating effect of natural genetic variation by combining behavioral (2511 focal hours), endocrine (366 urine samples) and genetic data (46 target regions in 21 HPA axis genes) from wild male Assamese macaques. A genetic risk score, calculated as the proportion of missense alleles an individual carried, was positively associated with average immunoreactive urinary cortisol levels, negatively with individual risk-taking behavior, and positively with social bond strength and bond maintenance behavior. Crucially, genetic risk modulated the social buffering effect on cortisol levels. The fact that social buffering cannot be put into effect at high genetic risk despite the high levels of affiliation observed among high risk individuals, suggests either that the salubrious effects of social bonds exceed the tight regulation of the HPA axis or that activation of the HPA axis is the trigger for support seeking behavior. Our study highlights that the physiological effects of affiliation vary between individuals, which may also affect the efficacy of social contact as a medical intervention. 


\section{Introduction}

Over the past decades evidence has mounted for social variables proximately affecting physiological processes including neuroendocrine, endocrine and immunological responses (Balasubramaniam et al., 2016; Cohen et al., 2007; Stephens \& Wallen, 2013; Uchino, 2006). Consequently, altered health outcomes ultimately affect longevity and reproduction which determine evolutionary fitness (Hawkley \& Capitanio, 2015; Holt-Lunstad et al., 2010; Ostner \& Schülke, 2018). One well-studied phenomenon is the social buffering effect whereby the presence of a close social partner attenuates stress responses of the hypothalamic-pituitary-adrenal (HPA) axis and the sympathetic nervous system (Cohen \& Wills, 1985). Despite its wide distribution across animal taxa and humans (Edgar et al., 2015; Hennessy et al., 2009; Hostinar et al., 2014; Kiyokawa \& Hennessy, 2018), social buffering is not ubiquitous among or within species. Whether a stress response can be attenuated will depend on the adaptive value of a particular type of partner when facing a particular type and intensity of stressor (Kiyokawa \& Hennessy, 2018). Yet, even field studies under ecologically valid conditions with tangible partner value leave unexplained a fair part of the variance in individual stress responses (Seyfarth et al., 2012; Wittig et al., 2016; Young et al., 2014a). Building on previous functional genetic work, we explore genetic variation of the HPA axis to explain more fully the individual variation in social buffering effects using wild male Assamese macaques (Macaca assamensis) as a model.

In wild Assamese and Barbary macaques (M. sylvanus), the strength of dyadic male social bonds predicts coalition formation and recruitment during fights (Schülke et al., 2010; Young et al., 2014b). These rather stable alliances (Kalbitz et al., 2016) serve in the acquisition and maintenance of high social status (Ostner et al., 2014; Schülke et al., 2010) which in turn predicts mating and reproductive success (Sukmak et al., 2014a; Young et al., 2013). Thus, maintaining a few close affiliative relationships provides functional support in aggressive conflicts and consequently male-male bonds attenuate the HPA axis response in Barbary macaques when faced with high rates of within-group aggression (Young et al., 2014a). Such social buffering effects can be modulated by single mutations in humans (Chen et al., 2011a) but the extent to which broader genetic 
variation explains parts of the variation in individual HPA axis activity remains unexplored.

We have developed a genetic panel of 46 regions in 21 genes known to be implicated in HPA axis functioning from work on humans and model organisms and designed primers for amplification and subsequent sequencing from non-invasive samples originating from field projects (Gutleb et al., 2018a, chapter 2). We identified 159 single nucleotide polymorphisms (SNPs) in a population of Assamese macaques and applied variant effect prediction to generate a genetic risk score (GRS, Belsky \& Israel, 2014; Worley et al., 2015) from 15 missense variants. The GRS is the proportion of an individual's alleles predicted to cause an amino acid change in the encoded protein (Gutleb et al., 2018a, chapter 2) and therefore likely to generate an altered phenotype. Here we combine this genetic work with behavioral and endocrine data on wild male Assamese macaques to assess how genetic risk modulates behavior and social buffering effects. 


\section{Results}

We built five linear regression models with GRS as one of the predictors which varied from 0 to $40 \%$ in all genotyped adult male and female individuals and from 0 to $17 \%$ in the adult males of this study (Figure 4.1A). We controlled for male dominance rank and used a data point per male for the mating and the non-mating season each, because responses and predictors vary between reproductive seasons and with male dominance rank (Kalbitz et al., 2016; Ostner et al., 2008a). In a simple model, mean immunoreactive urinary cortisol (iUC) levels across $8.6 \pm 2.6$ samples per male in the non-mating and $7.6 \pm 2.9$ samples per male in the mating season rose with increasing cumulative genetic risk $(\mathrm{t}=2.664, \mathrm{p}=0.011$, Table 4.1A, Figure 4.1C). A $17 \%$-increase of the GRS was associated with a $20.5 \%$ increase of the response variable iUC.

Male risk-taking behavior was assayed as the rate of initiating within-group aggression and differed between seasons from $1.06 \pm 0.45$ acts in the non-mating to 1.69 \pm 0.69 acts in the mating season (Wilcoxon signed-rank test: $n=22, p<0.001, z=-3.180$ ). Risk-taking decreased with increasing GRS after controlling for season and dominance rank $(\mathrm{t}=-2.513, \mathrm{p}=0.016$, Table 4.1B, Figure 4.1B). With a $17 \%$-increase of the GRS male risk-taking was reduced by $30.4 \%$. The relative rate of initiating and terminating close spatial proximity $(1.5 \mathrm{~m})$ with one's top two male partners was summarized in the Hinde index. The index was positively associated with GRS $(\mathrm{t}=2.772, \mathrm{p}=0.011$, Table 4.1C, Figure 4.1D) suggesting that the more risk alleles a male carried the more responsible he was for maintaining the social bonds with his top partners. A $17 \%$ increase of the GRS predicted a $306.8 \%$ increase in the relative investment into maintaining close proximity. The strengths of a male's social bonds with his top male partners assayed as the sum of his top two dyadic composite sociality index values (CSI after Schülke et al. 2010) tended to be $(\mathrm{p}<0.1)$ positively associated with GRS $(\mathrm{t}=1.750$, $\mathrm{p}=0.095$, Table 4.1D, Figure 4.1F). A $17 \%$-increase of the GRS was associated with a $28.6 \%$ increase of the response variable CSI.

To investigate whether GRS influenced the effect of social bonds on HPA axis activity, we built the buffering-model predicting mean iUC levels per male from the strength of his top two social bonds, his GRS, and the interaction of bond strength and 
GRS. The interaction between bond strength and GRS was significantly associated with iUC levels $(t=2.705, p=0.011$, Table $4.1 \mathrm{C})$. At the low end of genetic risk increasing CSI values were associated with decreasing HPA axis activation (iUC) suggestive of a social buffering effect. Yet, the higher the GRS was, the weaker was the social buffering effect (Figure 4.1E). These results and those of the maintenance model were robust against changing the number of top partners considered in calculating the sum of male's CSI values from two to three (Supplementary Table S3.1).

Table 4.1: Linear regression models

\begin{tabular}{|c|c|c|c|c|}
\hline Fixed effect & Estimate & Std. Error & t-value & p-value \\
\hline \multicolumn{5}{|c|}{$\begin{array}{l}\text { (i) iUC-model with log-transformed mean iUC levels as response. } \mathrm{n}=45 \text { data } \\
\text { points for } 23 \text { individuals and two seasons, significant difference to null model } \\
\mathrm{p}=0.006, \mathrm{R}^{2}=71.10 \%\end{array}$} \\
\hline Intercept & 4.061 & 0.191 & 21.248 & $<0.001$ \\
\hline Genetic risk & 0.049 & 0.019 & 2.664 & 0.011 \\
\hline Dominance rank & -0.077 & 0.115 & -0.672 & 0.506 \\
\hline Season: mating & 1.608 & 0.162 & 9.928 & $<0.001$ \\
\hline \multicolumn{5}{|c|}{$\begin{array}{l}\text { (ii) Risk-taking-model with square-root transformed rate of initiating } \\
\text { aggression as response. } \mathrm{n}=45 \text { data points for } 23 \text { individuals and two seasons, } \\
\text { significant difference to null model } \mathrm{p}=0.009, \mathrm{R}^{2}=39.60 \%\end{array}$} \\
\hline Intercept & 1.119 & 0.083 & 13.489 & $<0.001$ \\
\hline Genetic risk & -0.020 & 0.008 & -2.513 & 0.016 \\
\hline Dominance rank & 0.097 & 0.050 & 1.938 & 0.060 \\
\hline Season: mating & 0.281 & 0.070 & 4.002 & $<0.001$ \\
\hline \multicolumn{5}{|c|}{$\begin{array}{l}\text { (iii) Maintenance-model with Hinde index as response. } \mathrm{n}=88 \text { data points for } 23 \\
\text { individuals, two top partners and two seasons, significant difference to null } \\
\text { model } \mathrm{p}=0.015, \mathrm{R}^{2}=21.76 \%\end{array}$} \\
\hline Intercept & -8.977 & 6.471 & -1.387 & 0.175 \\
\hline Genetic risk & 1.620 & 0.585 & 2.772 & 0.011 \\
\hline Rank difference & 1.985 & 4.342 & 0.457 & 0.649 \\
\hline Season: mating & -2.335 & 4.344 & -0.538 & 0.593 \\
\hline \multicolumn{5}{|c|}{$\begin{array}{l}\text { (iv) Social bonding-model with square-root transformed sum of CSI with the } \\
\text { top two male partners as response. } \mathrm{n}=44 \text { data points for } 23 \text { individuals and two } \\
\text { seasons, trend for a difference to null model } \mathrm{p}=0.059, \mathrm{R}^{2}=45.28 \%\end{array}$} \\
\hline Intercept & 1.667 & 0.159 & 10.478 & $<0.001$ \\
\hline Genetic risk & 0.028 & 0.016 & 1.750 & 0.095 \\
\hline Dominance rank & 0.202 & 0.090 & 2.244 & 0.031 \\
\hline Season: mating & 0.011 & 0.110 & 0.097 & 0.924 \\
\hline \multicolumn{5}{|c|}{$\begin{array}{l}\text { v) Buffering-model with log-transformed mean iUC levels as response. } \mathrm{n}=44 \\
\text { data points for } 23 \text { individuals and two seasons, significant difference to null } \\
\text { model } \mathrm{p}=0.002, \mathrm{R}^{2}=83.36 \%\end{array}$} \\
\hline Intercept & 4.426 & 0.167 & 26.471 & $<0.001$ \\
\hline Genetic risk & 0.336 & 0.099 & 3.397 & 0.003 \\
\hline Sum of CSI & -0.320 & 0.103 & -3.110 & 0.004 \\
\hline Dominance rank & -0.023 & 0.124 & -0.188 & 0.852 \\
\hline Season: mating & 1.540 & 0.136 & 11.329 & $<0.001$ \\
\hline Genetic risk: sum of CSIs & 0.302 & 0.111 & 2.705 & 0.011 \\
\hline
\end{tabular}



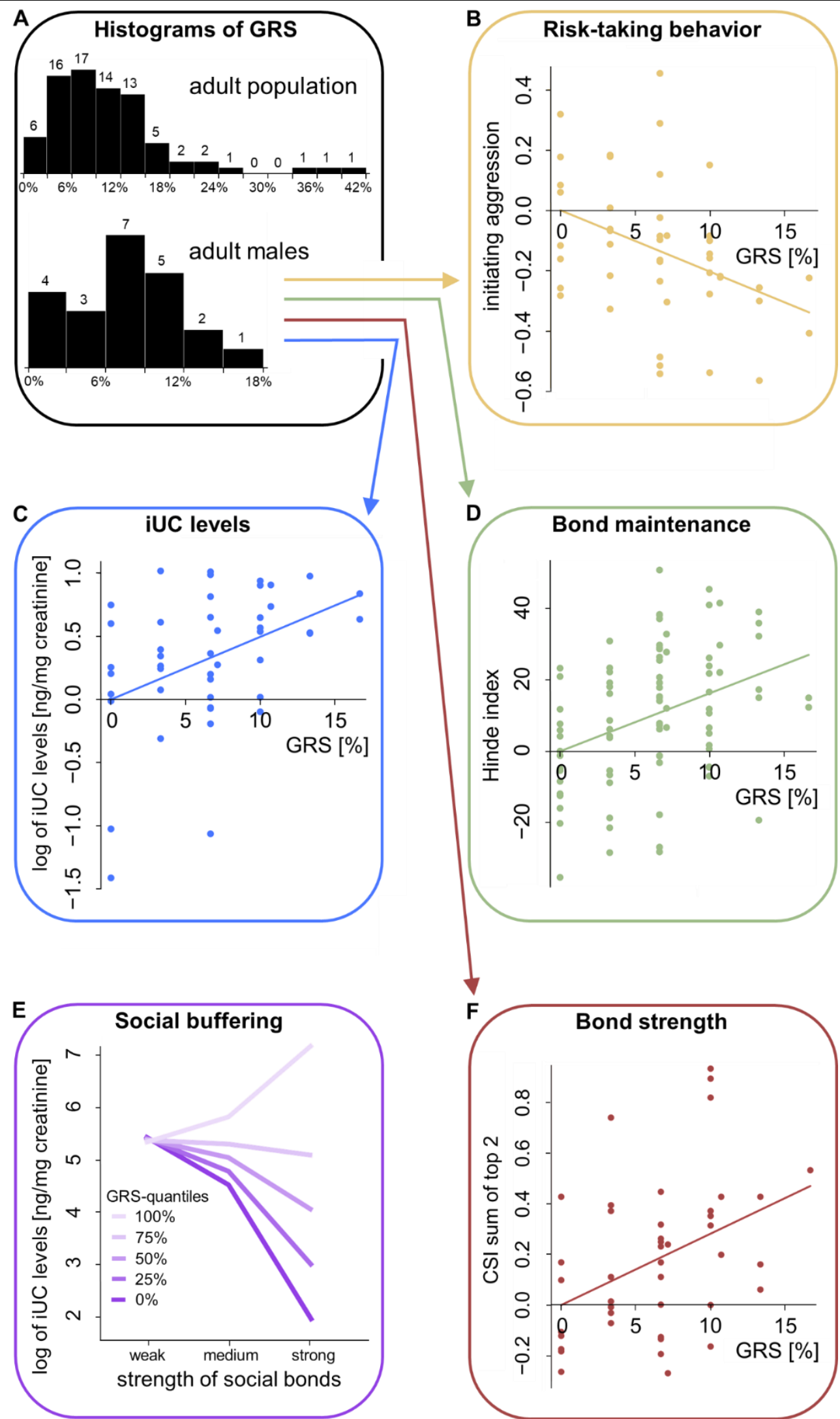

Figure 4.1: (A) Occurrence and distribution of the GRS among all genotyped adult male and female individuals and among adult males in this study. Increasing GRS of the HPA axis was associated in males with (C) increasing immunoreactive urinary cortisol levels (iUC), (B) decreasing risk-taking behavior that is lowered rates of initiating aggression, (D) increasing responsibility for maintaining proximity with the top two male partners that is the Hinde index and (F) increasing CSI with the top two male partners. (E) GRS interacts with CSI of the top two male partners to predict iUC levels; the effect that stronger bonds attenuate HPA axis activity is strongest at minimal GRS and wanes as GRS increases. Data points reflect partial residuals. 


\section{Discussion}

Functional genetic studies link DNA sequence variation in a number of genes from the serotonergic (Goodyer et al., 2009), catecholamine/dopaminergic (Bouma et al., 2012) and corticoid pathways (Mahon et al., 2013) to the activation and regulation of the HPA axis. The effects of such genetic variation concern gene regulation (Chen \& Miller, 2012), receptor functioning (Wedemeyer et al., 2007), enzyme activity (Chen et al., 2004) and effectively the production and secretion of cortisol (Velders et al., 2011). Variation in these individual genes has also been associated with behavioral phenotypes concerning anxiety (Lesch et al., 1996), risk-taking (Mata et al., 2012), aggression (Gutleb et al., 2018b, chapter 3), emotion (Pagliaccio et al., 2015) and social perception (Skuse \& Gallagher, 2011). Here we combine multi-locus genotyping of the natural variation in HPA axis related genes, variant effect prediction, non-invasive measures of HPA axis activity and behavioral phenotyping in a wild mammal.

The naturally occurring SNP variants were predicted to cause amino acid substitutions in the glucocorticoid receptor, catechol-O-methyl transferase, brain-derived neurotrophic factor, dopamine transporter, serotonin receptor, opioid receptor and neuropeptide Y. Amongst others, these products are responsible for information processing, neurotransmitter degradation, removal and inactivation and have been associated with HPA axis functioning, cortisol levels and stress vulnerability (Gutleb et al., 2018a, chapter 2). As a proof of principle, we show with our small sample of male macaques that a cumulative risk score in these genes is associated with levels of cortisol, the end product of the HPA axis. The relationship between GRS and iUC seems to be driven by the invariably positive residual iUC levels of individuals with GRS $\geq 10 \%$ equivalent to three or more alleles predicted to cause amino acid changes. Similarly, all associations between GRS and risk-taking, bond maintenance and bond strength were driven by behavior of males with GRS $\geq 10 \%$. At low to no risk in HPA related genes all responses varied more widely, suggesting that other factors besides genetic risk were also at play.

Factors driving variation in HPA axis activity and behavior include the social and the ecological environment, with food abundance, reproductive season and dominance 
rank being the most prominent examples in primates (Beehner \& Bergman, 2017; Goymann et al., 2004; Pride, 2005; Sapolsky, 2005). In line with previous studies (Beehner \& Bergman, 2017; Ostner et al., 2008a; Young et al., 2014a), HPA axis activity and risk-taking behavior increased during the mating season. Reproductive season did not influence proximity maintenance and the top social bond strength though, because strong bonds in Assamese macaque males and other primates are characterized by their stability over time (Silk et al., 2010b). The relationship between dominance and HPA activity is highly variable across and within primate species (Cavigelli \& Caruso, 2015), possibly as a function of variation in allostatic load associated with attaining and maintaining high dominance status (Goymann et al., 2004; Sapolsky, 2005) and was not significant here. Since we statistically controlled for the effects of dominance rank and season, these factors cannot explain the pronounced variation in HPA activity at low to no genetic risk.

The social buffering framework (Cohen \& Wills, 1985) provides a potential explanation for such residual variation. Accordingly, the presence of or affiliative interaction with an important social partner attenuates HPA axis activity in general or in response to stressful events (Kiyokawa \& Hennessy, 2018). In wild and captive groupliving primates these salubrious effects of bonding are provided not only by a mother to her offspring (Sanchez et al., 2015) but also by strongly bonded same or opposite sex social partners (Galvão-Coelho et al., 2012; Gunnar et al., 1980; Winslow et al., 2003; Wittig et al., 2016; Young et al. 2014a).

In line with a previous study showing that a single SNP can influence the effect of social support on the HPA axis response in humans (Chen et al., 2011a), we demonstrate that the buffering effect of social bonds disappears with increasing GRS. Put the other way around, the pronounced variation in iUC and behavior at low to no genetic risk was explained by individual variation in social bonding and the resulting social buffering of the HPA axis.

If at high GRS social buffering is hampered due to an altered HPA axis regulation, why would high risk individuals establish relatively strong social ties and invest even more heavily in maintaining contact with their closest partner than individuals with a lower genetic risk? On the physiological level, social bonds attenuate not only the HPA axis activation but can also affect cardiovascular, neural and immune reactions to stressors (Balasubramaniam et al., 2016; Lutgendorf et al., 2000; McCowan et al., 2016; 
Pressman et al., 2005; Uchino, 2006). If these effects are not fully mediated via HPA regulation, individuals with altered HPA axis genes may still benefit from social buffering on other systems. Additionally, if the tendency to form close social bonds evolved in response to the ubiquitous competition that is inherent in group living, strong social bonds may convey functional support via agonistic aid in actual conflicts (Berghänel et al., 2011; Mitani et al., 2000; Schino, 2007), tolerance in competition for resources (Haunhorst et al., 2017; Tiddi et al., 2011) and safety from predators (Josephs et al., 2016; Ostner \& Schülke, 2018) regardless of the social buffering function of bonding.

We are left to explain why increasing GRS is also associated with increasing responsibility for maintaining close proximity with the strongest partners, as all males regardless of GRS should invest in social bonds. In human children, contact with a bonded partner after stress-induced HPA axis activation accelerates the return to baseline (Seltzer et al., 2010). If support seeking as a coping strategy (Taylor et al., 2000) is triggered by elevated cortisol levels, high GRS leading to high iUC levels might drive individuals to seek contact with their bonded partners more than low GRS males because this contact does not help to downregulate cortisol either. This relationship is not found in female chacma baboons (Papio ursinus) where a personality dimension ('loner') is positively associated with fecal glucocorticoid metabolite levels similar to the GRS in our study (Seyfarth et al., 2012). Yet the personality dimension is negatively associated with the strength of a female's closest bonds, indicating that those with high cortisol levels do not seek more contact perhaps because of constraints to supportive social bonding in a group where most competition is among group members (Ostner \& Schülke, 2014). Experimentally, both attenuated HPA axis responses and accelerated returns to baseline cortisol levels have been demonstrated (Gunnar \& Hostinar, 2015; Kiyokawa \& Hennessy, 2018). The relative importance of both mechanisms will depend on the temporal patterning of the stressor (immediate short aggressive interaction versus lasting variation in competition for access to resources leading to energetic stress) and the spatiotemporal association with the supporter (always close before or during exposure or not).

Similar to our findings on the variation in social bonding and risk-taking behavior, natural genetic variation in other macaque species has been associated with social network position (Brent et al., 2013), vigilance to threat (Watson et al., 2015), rates of aggression (Gutleb et al., 2018b, chapter 3), timing of natal dispersal (Krawczak et al., 
2005; Trefilov et al., 2000) and personality traits (Brent et al., 2014) suggesting a significant portion of individual variation in social behavior to be independent from external ecological and social factors. Apart from DNA sequence variation also gene expression patterns of macaques have been linked with (experimentally manipulated) dominance rank (Tung et al., 2012) and grooming behavior (Snyder-Mackler et al., 2016b) with effects being partly mediated by cortisol signaling (Tung et al., 2012) and with important consequences for immune regulation and responses to infection (SnyderMackler et al., 2016b).

Social buffering of the stress response is a widespread phenomenon in humans, non-human primates and other animals with the potential to fully mitigate adverse effects of overly high or exceedingly long stress responses (Gunnar et al., 1980; Levine et al., 1985; Tzeng et al., 2018). Social buffering is not ubiquitous though. Whether the effect of a particular stressor can be buffered by a specific type of partner depends on whether such partners provide adaptive benefits under ecologically valid conditions (Gunnar \& Hostinar, 2015; Kiyokawa \& Hennessy, 2018). Yet even if the adaptive value of social support is given, natural genetic variation might modulate social buffering effects leading also to variation in social behavior. Our findings suggest that the genetic make-up in HPA axis related genes explains a significant part of the variation seen in the effect of social bonding on individuals' stress responsiveness. If the same applies to humans, we foresee that the effectiveness of social coping mechanisms to stress varies with the genetic background of the individual. This should be taken into account when judging the value of specific behavioral therapies aiming to help people in coping with stressful events using mechanisms of social support. 


\section{Materials and methods}

\section{Ethics statement}

Behavioral observations as well as feces and urine collection were conducted noninvasively. We followed the American Society of Primatologists principles for the ethical treatment of non-human primates and the guidelines of the Animal Welfare Body of the German Primate Center. The Department of National Parks, Wildlife and Plant Conservation and the National Research Council of Thailand authorized the data collection and sample-export with a benefit sharing agreement (permit numbers: 0004.3/3618, 0002.3/2647, 0002/17, 0002/626, 0002/2424).

\section{Study population}

This study was conducted at the Phu Khieo Wildlife Sanctuary in Thailand (Schülke et al., 2011). It is part of the $6500 \mathrm{~km}^{2}$ protected Western Isaan Forest Complex and provides a variety of terrestrial ecoregions for a diverse community of large mammals and predators (Borries et al., 2002; Grassman et al., 2005). We followed four habituated groups of Assamese macaques and selected all 25 adult males present in these groups as study subjects. Relatedness among males was unknown but expected to be low because all males disperse from their natal group before reaching sexual maturity and repeatedly change groups thereafter. The area was densely populated with additional unhabituated groups serving as targets and sources of male migration.

At the beginning of our study period group sizes were 55 with 9 adult males and 13 adult females (ASM), 28 with 3 adult males and 9 adult females (ASS), 41 with 9 adult males and 10 adult females (AOM) and 21 with 4 adult males and 6 adult females (AOS). Two adult males from AOM died after two months and five adult males changed groups between the non-mating and the mating season $\left(11^{\text {th }}\right.$ of September to $20^{\text {th }}$ of October). This time period and the two deceased males were excluded from data analyses. 


\section{Data collection}

About $5 \mathrm{~g}$ of feces were collected directly after defecation into $50 \mathrm{ml}$ tubes (62.559.001, Sarstedt, Nümbrecht, North Rhine-Westphalia, Germany) containing $30 \mathrm{ml}$ of $97 \%$ ethanol. After storage for 24 to 36 hours the solid material was transferred into another $50 \mathrm{ml}$ tube containing $30 \mathrm{ml}$ of silica beads for dark cool storage until export to Germany (Nsubuga et al., 2004; Roeder et al., 2004). Following arrival at the laboratory, samples were frozen at $-20^{\circ} \mathrm{C}$ until DNA extraction was performed at the Primate Genetics Laboratory at the German Primate Center.

Samples for iUC quantification were collected immediately after urination from 25 individually known adult males between March 2015 and February 2016. On average we analyzed $8.6 \pm 2.6(2-14)$ urine samples per male during the non-mating season (March-September, $\mathrm{n}=198)$ and $7.6 \pm 2.9$ (2-13) samples during the mating season (October-February, $n=168$ ). Urine was collected using salivettes designed for the use in salivary cortisol analysis (51.1534.500, Sarstedt AG \& Co., Nümbrecht, North RhineWestphalia, Germany). The method has been recently validated for non-invasive urine sampling from free-ranging animals (Danish et al., 2015) and has been used successfully for the measurements of various physiological markers in other macaque species (Danish et al., 2015; Müller et al., 2017). To collect urine, disposable gloves were used and one end of the salivette swab was dipped into urine drops on leaves or the forest floor to soak them up (Danish et al., 2015). Only urine uncontaminated with fecal matter was taken. Upon return to the laboratory of the field station, the urine was recovered from the collection device by centrifuging the salivettes at $6000 \mathrm{rpm}$ for 5 minutes. The recovered urine was subsequently transferred to $2 \mathrm{ml}$ polypropylene tubes (0030120094, Eppendorf AG, Hamburg, Germany) prior to freezing at $-20{ }^{\circ} \mathrm{C}$. All urine samples were shipped to the Endocrinology Laboratory at the German Primate Center on dry ice and stored frozen at $-20^{\circ} \mathrm{C}$ until analysis.

We collected behavioral data from 25 adult males between March 2015 and February 2016, thus including an entire non-mating (March to September) and the consecutive mating season (October to February, Fürtbauer et al., 2010). During 40 minutes focal animal observations, conducted between dawn and dusk, all affiliative, 
agonistic and sexual interactions were recorded continuously (Martin \& Bateson, 1993). We made an effort to balance focal animal protocols across different males and the time of day (mean \pm SD $98 \pm 24$ hours/male, total $=2511$ hours). To establish the dominance hierarchy additional ad libitum data were collected on agonistic interactions.

\section{Data analyses}

After DNA extraction of fecal samples collected from all 38 adult males and 41 adult females in the field project (including deceased and emigrated individuals) using the First-DNA all-tissue Kit (D1002000, GEN-IAL GmbH, Troisdorf, North RhineWestphalia, Germany), we applied our HPA axis genotyping panel that was described in detail elsewhere (Gutleb et al., 2018a, chapter 2, Sequence Read Archive SRP116685). The genotyping panel contains 46 target regions in 21 genes: $A V P R 1 B$ arginine vasopressin receptor $1 \mathrm{~B}, B D N F$ brain-derived neurotrophic factor, COMT catechol-Omethyl transferase, $C R H$ corticotropin-releasing hormone, $C R H B P$ corticotropin releasing hormone binding protein, CRHR1 corticotropin-releasing hormone receptor $1, C R H R 2$ corticotropin-releasing hormone receptor 2, DRD3 dopamine receptor D3, FKBP5 FK506 binding protein $5, H T R 1 A$ serotonin receptor $1 \mathrm{~A}, H T R 1 B$ serotonin receptor $1 \mathrm{~B}$, MAOA monoamine oxidase A, $M C 2 R$ adrenocorticotropic hormone receptor, $N P Y$ neuropeptide $\mathrm{Y}, N R 3 C 1$ glucocorticoid receptor, OPRM1 opioid receptor mu 1, OXTR oxytocin receptor, SERPINA6 corticosteroid binding globulin, SLC6A3 dopamine transporter, SLC6A4 serotonin transporter and TPH2 tryptophan hydroxylase 2 (see Gutleb et al., 2018a for references and detailed information on PCR setup, primers and cycling conditions).

Sequencing libraries were prepared following previously described methods (Rohland et al., 2015) without uracil-DNA-glycosylase treatment based on Meyer and Kircher (2010) and Kircher et al. (2012). After samples were submitted to Illumina NGS (MiSeq paired-end $150 \mathrm{bp}$ ), the bioinformatics analyses of the acquired NGS-data included an improved pipeline, ensuring that polymorphisms are identified by two different variant calling approaches (GATK and SAMtools, Li et al., 2009; McKenna et al., 2010) and classified according to their impact by two different variant effect 
prediction pipelines (VEP and SnpEff, Cingolani et al., 2012; McLaren et al., 2016). With the rhesus macaque genome (rheMac8) as reference we identified 159 SNPs among the 79 adult Assamese macaques, for which both VEP and SnpEff predicted the same variant consequences/impact classes (Gutleb et al., 2018a, chapter 2). We called the 15 SNPs of the 'moderate impact' class 'risk SNPs'. All of them were missense variants and classified to cause moderate effects according to the rhesus macaque genome (Table 4.2) and were the rarer alleles in our study population. We calculated a GRS for each individual as the proportion of alleles that were risk alleles at the 13-15 loci genotyped per individual (Ø 14.8).

Table 4.2: Gene affiliation, location and missense variants of SNPs in the GRS

\begin{tabular}{|c|c|c|}
\hline Gene & Position & Codons and amino acids \\
\hline $\begin{array}{l}\text { dopamine transporter } \\
(S L C 6 A 3)\end{array}$ & 1332856 & $\begin{array}{l}\text { gGa/gAa } \\
\text { glycine/glutamic acid }\end{array}$ \\
\hline $\begin{array}{l}\text { brain-derived neurotrophic factor } \\
(B D N F)\end{array}$ & 38305600 & $\begin{array}{l}\text { gGt/gAt } \\
\text { glycine/aspartic acid }\end{array}$ \\
\hline $\begin{array}{l}\text { catechol-O-methyl transferase } \\
(C O M T)\end{array}$ & 61692389 & $\begin{array}{l}\text { Atg/Gtg } \\
\text { methionine/valine }\end{array}$ \\
\hline $\begin{array}{l}\text { serotonin receptor } 1 \mathrm{~B} \\
(H T R l B)\end{array}$ & 75621679 & $\begin{array}{l}\text { Ggg/Agg } \\
\text { glycine/arginine }\end{array}$ \\
\hline & 75621805 & $\begin{array}{l}\text { Ccc/Gcc } \\
\text { proline/alanine }\end{array}$ \\
\hline $\begin{array}{l}\text { neuropeptide } \mathrm{Y} \\
(N P Y)\end{array}$ & 83926991 & $\begin{array}{l}\mathrm{gAc} / \mathrm{gGc} \\
\text { aspartic acid/glycine }\end{array}$ \\
\hline & 83927111 & $\begin{array}{l}\text { aGc/aAc } \\
\text { serine/asparagine }\end{array}$ \\
\hline $\begin{array}{l}\text { opioid receptor mu } 1 \\
(O P R M 1)\end{array}$ & 112074108 & $\begin{array}{l}\mathrm{ttG} / \mathrm{ttC} \\
\text { leucine/phenylalanine }\end{array}$ \\
\hline \multirow{7}{*}{$\begin{array}{l}\text { glucocorticoid receptor } \\
(N R 3 C 1)\end{array}$} & 112074125 & $\begin{array}{l}\text { Ggt/Agt } \\
\text { glycine/serine }\end{array}$ \\
\hline & 112074164 & $\begin{array}{l}\mathrm{Gcc} / \mathrm{Ccc} \\
\text { alanine/proline }\end{array}$ \\
\hline & 141200981 & $\begin{array}{l}\text { Aca/Tca } \\
\text { threonine/serine }\end{array}$ \\
\hline & 141200990 & $\begin{array}{l}\mathrm{Cct} / \mathrm{Tct} \\
\text { proline/serine }\end{array}$ \\
\hline & 141202027 & $\begin{array}{l}\mathrm{aCt} / \mathrm{aTt} \\
\text { threonine/isoleucine }\end{array}$ \\
\hline & 141202066 & $\begin{array}{l}\text { aAt/aGt } \\
\text { asparagine/serine }\end{array}$ \\
\hline & 141202114 & $\begin{array}{l}\mathrm{cCc} / \mathrm{cTc} \\
\text { proline/leucine }\end{array}$ \\
\hline
\end{tabular}

Note: Position refers to the chromosome location in the rhesus macaque genome rheMac8. Capital letters in the codons mark the SNPs. 
Immunoreactive urinary cortisol (iUC) concentrations were determined by microtiter plate enzyme immunoassays using an antiserum against cortisol-3-CMO:BSA and biotinylated cortisol as enzyme conjugate (Palme \& Möstl, 1997). Prior to analysis, urine samples were diluted 1:10 to 1:10.000 (depending on concentration) in assay buffer and duplicate $50 \mu 1$ aliquots of diluted samples and cortisol standard (50 $\mu 1,0.6$ - 40 $\mathrm{pg} / 50 \mu \mathrm{l})$ were combined with labelled cortisol $(50 \mu \mathrm{l})$ and antiserum $(50 \mu \mathrm{l})$ and incubated overnight at $4{ }^{\circ} \mathrm{C}$. After incubation, the plates were washed four times, $150 \mu \mathrm{l}$ ( 7 ng) of streptavidin-peroxidase (S5512; Sigma-Aldrich Chemie GmbH, Bavaria, Germany) in assay buffer was added to each well and the plates incubated at room temperature in the dark for $60 \mathrm{~min}$ and then washed again four times. TMB substrate solution (100 $\mu$ l; 1-Step Ultra TMB, Thermo Fisher Scientific Inc., Rockford, USA) was subsequently added and the plates incubated at room temperature in the dark for another 45 - 60 min. The enzyme reaction was finally stopped by adding $50 \mu \mathrm{l}$ of $2 \mathrm{M} \mathrm{H}_{2} \mathrm{SO}_{4}$ to each well and absorbance measured at $450 \mathrm{~nm}$ (reference $630 \mathrm{~nm}$ ) in a spectrophotometer. Sensitivity of the assay at $90 \%$ binding was $0.5 \mathrm{pg}$. Serial dilutions of urine from different males showed displacement curves parallel to the cortisol standard curve with no differences in slopes (test for equality of slopes: $\mathrm{F}_{1,8}=0.0585-0.3590$; all $\mathrm{p}>0.5)$. Intraassay coefficients of variation of high and low value quality controls were $5.8 \%$ and $8.4 \%$, respectively, while respective figures for interassay variation were 9.7 $\%$ and $12.5 \%$. To adjust for variations in the dilution of the voided urine, iUC concentrations were indexed against urinary creatinine $(\mathrm{Cr})$, measured as described by Bahr et al. (2000) and expressed as ng iUC/mg Cr. For the statistical analyses we calculated a mean iUC value for each individual during the non-mating and the mating season each.

From focal animal protocols we measured risk-taking as the hourly rate of initiating aggression for each individual during the non-mating and the mating season. We counted every observation of the focal animal starting an aggressive interaction with any other group member (i.e. bite, chase, check-look, choose-position, growl, groundslap, head-bob, lunge, open-mouth, peer, pretend-grab, point, push-pull, repeated growl/scream/yell, slap and stare).

All decided dyadic agonistic interactions from the continuous and the ad libitum data, where clear submissive behavior (i.e. silent-bared teeth display, make room and unprovoked give ground) was observed only by one male in a conflict were used to create 
a hierarchy matrix (Gutleb et al., 2018b, chapter 3; Haunhorst et al., 2017, Supplementary Table S3.2). We calculated hierarchies separately for the non-mating and the mating season (due to males changing group) with the package DomiCalc (Schmid \& de Vries, 2013). 'Combil' ordinal ranks (a combination of two DomiCalc indices, PD and DomSub) were standardized to scale between -1 (lowest-ranking) and 1 (highest-ranking) to allow a comparison between differently sized groups.

We calculated a composite sociality index (CSI) to measure the strength of affiliative relationships between two males relative to the average male-male relationship in each group separately for the non-mating and the mating season (Silk et al. 2006a). We included both the duration and the frequency of three behaviors: being in close $(1.5 \mathrm{~m})$ proximity, body contact, and grooming for each dyad (Supplementary material). The duration of grooming was subtracted from the duration of body contact and the duration of body contact was subtracted from the duration of close proximity, because they are nested into each other (Haunhorst et al., 2016; Kalbitz et al., 2016). All behaviors were positively correlated to each other in row-wise matrix correlations at $\mathrm{p}<0.05$ and an average rho of $0.61(0.32-0.89)$.

We calculated the Hinde index (Hinde \& Atkinson, 1970) of approaches into and departures from a radius of $1.5 \mathrm{~m}$ to measure responsibility for the maintenance of close spatial proximity (Supplementary material).

Statistical analyses were conducted using R 3.1.2 (The R Foundation for Statistical Computing, Vienna, Austria, http://www.r-project.org). For all analyses, we ran general linear models including season and social group as control factors and animal ID as a random factor. We included season, because it has previously been shown that glucocorticoid hormones and aggression increase during the mating season (Gutleb et al., 2018b, chapter 3; Ostner et al., 2008a). Dominance rank was included as an additional control variable in the iUC-, the risk-taking and the social bonding-model and absolute dominance rank difference as an additional control variable in the maintenance-model. To achieve normal distribution, iUC was log-transformed and the rate of initiating aggression and sum of CSI with top two male partners was square-root transformed. In the bufferingmodel the GRS and the CSI-sum were z-transformed. 
All models were calculated with the package 'ImerTest' with alpha levels set to 0.05 (Bates et al., 2015; Kuznetsova et al., 2017). Various model diagnostics were applied to confirm model validity: visual inspection of distribution of residuals, qqplots, residuals plotted against fitted values, assessing model stability using the function 'glmm stability' written by Roger Mundry (MPI Evolutionary Anthropology, Leipzig, Germany), assessing leverage and dfbetas for single samples and levels of the random factor animal ID using the package 'influence.ME' (Niewenhuis et al., 2013), and variance inflation factors using the package 'car' (Fox \& Weisberg, 2011). None of the diagnostics suggested a violation of the model assumptions. All variance inflation factors were below two, indicating that collinearity between predictors did not affect the results.

The full models were significantly different from the null models (excluding GRS and CSI in the buffering-model and GRS in all other models; likelihood ratio test with the $\mathrm{R}$ function 'anova', setting the argument to 'Chisq') in all but the social bonding-model in which $0.1<\mathrm{p}<0.05$.

\section{Acknowledgements}

We thank the National Research Council of Thailand and the Department of National Parks, Wildlife and Plant Conservation for permission to conduct this study and for all the support granted. We are grateful to J. Prabnasuk, K. Nitaya T. Wonsnak, M. Pongjantarasatien and K. Kreetiyutanont, M. Kumsuk, W. Saenphala from Phu Khieo Wildlife Sanctuary for their cooperation over the years and permission to carry out this study. We thank A. Koenig and C. Borries, who established the field site. Special thanks go to S. Jumrudwong, W. Nueorngshiyos, N. Juntuch, J. Wanart, R. Intalo, T. Kilawit, N. Pongangan, B. Klaewklar, N. Bualeng, A. Ebenau, P. Saisawatdikul, K. Srithorn, M. Swagemakers and T. Wisate for their excellent help in the field. We acknowledge C. Schwarz, N. Westphal and F. Aron for their support in the genetics lab and M. Polten for her assistance in hormone analysis. We also thank H. Sennhenn-Reulen and N. Müller for statistical advice and T. S. Gutleb for helpful comments on the manuscript. We thank the members of the research unit 'Sociality and Health in Primates' (DFG FOR 2136) for stimulating discussions. This research was funded by the Deutsche Forschungsgemeinschaft (DFG, German Research Foundation) - project number OS201/8-1; 254142454/GRK 2070. The authors declare no conflict of interest. 


\section{Chapter 5}

\section{General discussion}


In chapter 1, I gave a general introduction to the topic and background of my thesis. In chapter 2 (study 1), I designed a genotyping panel useful for research on the genetics of stress and aggression. In chapter 3 (study 2), I demonstrated that the widely studied human COMT $\mathrm{Val}^{158} \mathrm{Met}$ polymorphism is not unique to humans and that it can be associated with similar phenotypes in a non-human primate. In chapter 4 (study 3), I integrated information from all missense variants detected in HPA-axis-related genes to calculate a GRS for each adult male and combined it with detailed endocrine and behavioral data. In the general discussion, I will first summarize the main results of the three preceding chapters, then discuss the results and provide avenues for future research.

\subsection{Summary of the results}

The results of the methodological study in chapter 2 are (i) the compilation of a list of presumably functional target regions in genes that are involved in the HPA-axis, (ii) the design of a high-throughput genotyping panel suitable for investigating the factors contributing to HPA-axis-related phenotypes, (iii) the demonstration that the panel is applicable to low-quality DNA, such as that obtained from fecal samples, which are often the only available sample material from wild animal populations and (iv) the demonstration that polymorphisms at purportedly functional HPA-axis loci exist in a natural primate population. Study 1 (chapter 2) describes the methods to include genotypic information by applying state-of-the-art sequencing technologies to fecal DNA samples. It served as the basic methodology necessary to conduct study 2 and 3 (chapter 3 and 4) and should motivate field biologists to include multi-locus genotype data in studies on wild, non-model populations more frequently.

The results of chapter 3 (study 2 ) are that (i) the widely studied human COMT $\mathrm{Val}^{158} \mathrm{Met}$ polymorphism occurs in a non-human primate species with genotype frequencies comparable to human populations, (ii) macaques' aggression rates increased with dominance rank in Val/Val individuals, but decreased in individuals carrying other genotypes and (iii) when changing from a lower to a higher dominance rank position, Val/Val individuals decreased, whereas Met/Met individuals increased their aggression 
rate. Now that I demonstrated that the COMT $\mathrm{Val}^{158} \mathrm{Met}$ polymorphism is not unique to humans and predicts similar behavioral phenotypes in another species, follow-up studies on this polymorphism in several species are called for to investigate the distribution of this polymorphism among taxa and help to decipher its evolutionary roots and contribution to individual variation in aggressive behavior.

The results of chapter 4 (study 3) are that an increasing GRS, measured as the proportion of missense variants detected with the method described in chapter 2 (study 1), was associated with (i) increasing HPA-axis activity, (ii) decreasing risk-taking behavior, i.e. initiating aggression, (iii) a decreasing main social buffering effect, (iv) a trend to have stronger social bonds and (v) an increasing maintenance of close proximity with strong partners. These results suggest that significant proportions of the observed interindividual variation can be assigned to underlying genotypic variation in the HPA-axis system. The investigation of the main effect hypothesis of social buffering revealed a significant interaction effect between social bond strength and GRS on HPA-axis activity. The association between strong social bonds and low cortisol levels seems to diminish when moving from low GRS to high GRS, indicating that social buffering is in effect in individuals at the lower end of genetic risk, but not at the higher end of genetic risk. The results of chapter 4 (study 3 ) indicate that male macaques follow different social strategies and build a basis for future research considering genotype as a mediator of variation in HPA-axis activity including social buffering in a wide range of animal taxa. However, the results are preliminary, as relatedness among the study animals could account for the detected associations but was not yet included in the models. Further analyses are necessary to clarify whether the detected associations are due to variation in HPA-axis genes or other genome-wide similarities among kin. 


\subsection{Limitations of the studies and the need for technological advances}

Applying a novel approach to investigate the effects of HPA-axis-related genotype on behavioral and endocrine phenotypes in wild primates and using only non-invasively collected samples for molecular analyses causes some inherent limitations for the studies. I demonstrated that genotype has sizeable effects on phenotypic variation in a non-model population living under natural selection pressures and suggest that such genotypic influences should be taken into account in future studies investigating the causes of individual variation. Nevertheless, some of the findings are preliminary and should be interpreted with caution. With the studies of this thesis I hope to stimulate a more frequent consideration of genotype in biological field studies, especially in the field of primatology, but future studies pursuing this strategy may improve some aspects.

\subsubsection{Limitations}

First, the sample size was rather small for a behavioral genetics study, as such studies typically have sample sizes ranging from a few dozens to tens of thousands. Human studies often have access to big data and can use large data sets which are not available to field behavioral biologists. In scientific disciplines, where research is experimental, hypothesis-driven and detailed data are necessary, sample sizes in behavioral genetic studies tend to be smaller than in the often hypothesis-neutral, datadriven big data applications (e.g. Mazzocchi, 2015; Plomin et al., 2016). The detailed data collection in this thesis was strictly non-invasive and included four habituated groups of Assamese macaques resulting in a medium to large sample size for a field study in wild primates. Therefore, an increase of the sample size will be hard to accomplish also in future studies, at least regarding wild primates. As studies with small sample sizes are at risk to detect false positives, replication studies are highly valued particularly in the field of behavioral genetics (Plomin et al., 2016). Regarding the sample size of the selected target regions, the approach cannot keep up with whole-genome approaches, but the number of targeted regions and the amount of SNPs that entered the GRS are comparable 
to some human studies (e.g. 4 in Worley et al., 2015 and Di Iorio et al., 2017; 6 in Utge et al., 2018; 7 in Ferguson et al., 2012; 9 in Kathiresan et al., 2008; 10 in Chen et al., 2011b; 13 in Ripatti et al., 2010; 116 in Morrison et al., 2007). There is no access to high-quality DNA samples that could be used for whole-genome sequencing from several wild animal populations, therefore the approach to combine several candidate genes in an NGS-panel to include several gene loci presumably contributing to the phenotypes of interest represents an important first step to study behavioral genetics in the wild.

Second, in this thesis I did not control for kinship, although putative relatives were included in the sample. Population relatedness that is not accounted for could create false associations between genetic variation and phenotypic outcome. As close relatives will have more similar genotypes not only at the target loci, but also genome-wide, it is possible that associations between genotype and HPA-axis activity and behavior are due to genetic differences in other parts of the genome that are shared among relatives. Due to the matrilineal structure of the macaques, controlling for kinship would be of particular interest when working with data on females. Due to male migration, this concern might be negligible when working with data on males, but to find out whether the results withstand, it is essential to control for relatedness structure in the future. This could be accomplished by other molecular methods applicable to low-quality DNA samples, e.g. microsatellite markers or capture-based genome-wide sequencing (Queller et al., 1993; Snyder-Mackler et al., 2016a; Sukmak et al., 2014b). This would allow to run so-called statistical "animal models", in which relatedness can be controlled for (Kruuk, 2004). The animal model is a type of mixed model (linear regression with fixed and random effects) in which a kinship matrix can enter the model additionally. In this case, the random effect of the individual is not treated as independent but uses the knowledge from the kinship matrix to account for relatedness. When genotype then enters the model as a fixed effect, the relatedness among all individual dyads is taken into account.

Third, GRSs are based on the assumption that genetic effects are additive. This assumption might introduce an error due to neglecting i) that some SNPs might have opposing effects on physiology whereby their impacts are cancelled out and ii) the interaction between loci, called epistasis (Belsky \& Israel, 2014; Zuk et al., 2012). There is still little understanding about epistatic interactions, so it has been recommended to ignore their effects in the calculation of GRSs so far, unless interactions are known 
(Belsky \& Israel, 2014). If identified in the future, they can be incorporated into the GRSs. However, research on epistasis is still in its infancy and a reasonable application to non-model species will therefore still take some time.

Fourth, considering only the DNA sequence level may not be enough to draw conclusions about genotypic effects on phenotypes. On a functional level, studies of genomics are followed by transcriptomics and proteomics. Every discipline has a value on its own and the combination of them has the potential to increase our knowledge of molecular mechanisms, processes and pathways (Manzoni et al., 2018). Currently, research on genes, transcripts and proteins is represented by fragmented niche groups working on these topics, which probably hinders a proactive information flow among scientists of the different disciplines (Manzoni et al., 2018). Researchers might tend to focus on the strengths of their own discipline and the pitfalls of the others. In this regard, transcriptomicists might argue that transcript information is necessary besides data on the DNA sequence level and proteomicists might argue that protein information is necessary besides transcript information and data on the DNA sequence level to allow for valid statements about the functionality of the protein in question. The important roles that also gene expression and/or protein levels play in the emergence of phenotypes should be acknowledged, but regarding the confinement to non-invasive samples in this thesis, transcript and protein analyses are hardly feasible at the current state of the art. Regarding the general neglect of genotypic data in studies on wild animal populations, the studies in this thesis represent an important first step to a better understanding of factors shaping inter-individual differences. Moreover, the field of genomics entails many advantages when working with rather small sample sizes and wild animals: the DNA sequence is the same across tissues, without any circadian, seasonal or age changes. This is a major concern in the two other molecular disciplines and would require a further separation of the already small sample size (Belsky \& Israel, 2014). DNA is an outstanding biomarker, especially due to three of its characteristics: its primacy (being determined already before birth), its stability (through time and space) and its scope of influence (DNA variation is associated with numerous phenotypic aspects, e.g. the first law of behavioral genetics - all human traits show significant and substantial genetic influence, Belsky \& Israel, 2014; Plomin et al., 2016; Plomin \& Deary, 2015; Turkheimer, 2000; Turkheimer \& Gottesman, 1991). 
Despite these caveats, the results of this thesis give first insights into the genetic predisposition of a range of HPA-axis-related phenotypic characteristics in wild Assamese macaques. Urinary cortisol levels, aggression rates, the strength and maintenance of social bonds and the attenuating effect of social bonds on HPA-axis activity seem to be at least partly determined by individuals' genetic constitutions.

\subsubsection{A brief comment on the need for technological advances}

The improvement of our knowledge of factors influencing individual HPA-axis phenotypes is constrained by available methods. Particularly, effective non-invasive genotyping methods have the potential to broaden the scope of application of behavioral genetics in basic research. Therefore, there is a constant need to keep developing broadly applicable state-of-the-art genotyping methods to investigate individual variation on the DNA sequence level.

The possibilities to apply high-throughput genotyping methodologies to noninvasive samples are advancing. Recently, Snyder-Mackler and colleagues (2016a) presented a capture-based method to generate genome-wide sequencing data from baboon fecal samples. As the method works with genotype probabilities, which can be an efficient way to decipher relatedness, the low coverage of certain gene regions makes it unfortunately a rather unsuitable tool for behavioral genetics. This thesis aimed at providing a novel genotyping panel, applicable to low-quality DNA samples and useful for HPA-axis related research. I applied next-generation amplicon sequencing instead of the common mtDNA-NGS and whole genome sequencing, using non-invasively collected fecal samples that can be stored easily and for a long time in the field. Whereas the capture-based method for fecal samples has the drawbacks of low coverage and working with genotype probabilities, the application of whole-genome sequencing would result in unevenly distributed gene regions covered in the individuals. Therefore, we conducted amplicon NGS to have a high coverage of the same gene regions and especially the regions of interest in all individuals. 
The HPA axis is a complex cascade with a multitude of molecules involved. Functional problems at any stage could alter the phenotypic outcome. In the future, studies on captive animals, from whom blood samples are available, could help to decipher the specific functional significance of each polymorphism. This information can then be used to make predictions for animals living under natural selection pressures in the wild. Further, genotyping panels, like the one presented in this thesis, can be constantly modified, updated and extended. In this manner, the panel could be updated according to upcoming publications on HPA-axis associations or extended to more target regions also including physiological pathways other than the HPA-axis.

The lack of replicability is still a growing problem existing in parallel to the longstanding aim to decipher the sets of genes involved in the emergence of complex phenotypic traits. In personality research, candidate genes did not withstand metaanalyses and it has been suggested that more likely a large number of genetic variants, each having a small effect on its own, cumulatively explain a substantial part of the observed variation (Penke \& Jokela, 2016). Even if the effects of single polymorphisms are rather small, collectively combined in a polygenic analysis with cumulative effect sizes and greater predictive power they constitute an important element to understand phenotypic variation. Analyzing SNPs jointly, genetic risk scores can associate with phenotypes even in the absence of single SNP associations (Traylor et al., 2016). Therefore, multi-locus approaches appear more promising as an effective tool to explain individual variation. In this approach, GRSs can be used to summarize the large number of small effects into one cumulative value. As Belsky and Israel (2014) pointed out, the now possible generation of high-throughput genetic data will probably lead to a spread of GRSs, especially in the field of social sciences. I suggest that this is also true for field behavioral biology, where large samples of whole genome data cannot be easily generated yet, but the use of multi-locus approaches invites to apply GRSs.

The results of this thesis add to a small but growing body of research that exemplifies the potential of behavioral genetics research to broaden our understanding of the variation observed in non-human primate populations (Di Fiore, 2003). Increasing the application of multi-locus genetic analyses in studies in the wild and identifying how much variation can be explained by genetic predisposition in natural, non-model populations, constitutes a major step in improving our understanding of animal 
characteristics (Brent \& Melin, 2014; Ekblom \& Galindo, 2011; Di Fiore, 2003; Tung et al., 2010). Steady methodological advancements are necessary to secure a further gain of knowledge in many areas of behavioral genetics, including the relationship between social bonds, individual stress levels, health, survival and fitness. 


\subsection{Effects of genotype on hormones and behavior in Assamese macaques}

The results of the studies in this thesis are summarized in chapter 5.1. In the following section I review possible interpretations of these results in light of the lives of Assamese macaques and discuss how genetic variants might be maintained in the population.

\subsubsection{How genetic variation shapes inter-individual differences}

Intra-specific differences in individuals' behavior and hormonal states are a compelling aspect of primate biology. Within the last years behavioral genetics targeting this inter-individual variation started to be implemented in non-human primates, with the majority of studies performed under laboratory conditions (Rogers, 2018). Functional genetic studies have been mainly conducted in captivity and test the genetic effects on responses to novel objects, to novel conspecifics and to social challenges (Rogers, 2018; Tung et al., 2010). Studies looking at the natural range of phenotypic variation in the wild are still scarce.

In this thesis, I applied two approaches to increase our knowledge on the genetic influences on phenotypes in wild Assamese macaques. After designing a sequencing panel suitable for the identification of gene variants from low-quality DNA samples, I followed i) a classical single variant candidate gene approach to elaborate on the extensively studied human COMT $\mathrm{Val}{ }^{158} \mathrm{Met}$ polymorphism and ii) a multi-locus approach with the focus on the calculation of a GRS from missense variants in a number of HPA-axis genes. Both approaches revealed significant genetic influences on the investigated parameters and thus provide new results on how genetic predisposition shapes inter-individual differences in Assamese macaques.

Recent findings suggest that genotypes interact with the environmental context to impact the probability of an individual expressing particular behaviors, including 
aggression (Conway et al., 2012; Gallardo-Pujol et al., 2013; Reif et al., 2007; Rutter et al., 2006; Shanahan \& Hofer, 2011; Simons et al., 2011). Concluding that some individuals are genetically predisposed to react differently to the same environmental conditions, this led to a strong increase in research on gene-environment effects in the human literature. One prominent candidate gene in such studies is COMT (Qayyum et al., 2015; Wagner et al., 2010). Consequently, I considered a moderating role of COMT $\mathrm{Val}^{158}$ Met on aggression dependent on differences in a salient social environmental condition of these primates - their dominance rank position. Indeed, in study 2 (chapter 3 ) I found that aggression rates vary with dominance rank dependent on the COMT Val ${ }^{158}$ Met genotype, which is suggestive of an underlying gene-environment interaction, with different social environments causing different behavioral outcomes dependent on the individual's genotype. Using individuals from a population living in their natural habitat, it was also possible to minimize further inter-individual variation of non-social environmental influences, which is difficult to control for in global human studies.

Based on the current findings of this thesis, I suggest two possible scenarios on how COMT genotype and rank contribute to individual aggression levels in Assamese macaques, which are not mutually exclusive. The first builds upon the assumption that being in a high rank position is more stressful or risky than being in a low rank position and probably bears a higher allostatic load (e.g. Goymann et al., 2004). In male Assamese macaques dominance rank is positively associated with paternity success and aggression is important to attain and maintain high social status (Schülke et al., 2010; Sukmak et al., 2014a). Low-ranking individuals benefit from ascending the hierarchy, so increased aggression may allow an individual to be perceived as present and involved in the group and thus to rise in rank by winning conflicts. According to the "warrior-worrier" model of the COMT Val ${ }^{158}$ Met polymorphism (Goldman et al., 2005; Stein et al., 2006), when being in a low rank position, the rather stress-resistant "warrior" Val/Val individuals might be able to afford expressing high aggression rates. However, when being in a high rank position already, further ascension is limited and thus a high aggression rate cannot lead to a further increase in rank. Aggression is then lower in Val/Val-individuals when being in a high rank compared to a low rank position. For the rather stress-sensitive "worrier" Met-carrying individuals the scenario would be the other way around. Metindividuals in a high rank position, and thus more stressful or risky situation, might be physiologically restricted and thus show high levels of aggression. While this explanation 
fits well for males, it remains puzzling for female macaques, in which dominance positions are usually determined by inheritance from the mother and thus less flexible than in males, making alliances less important for rank attainment and maintenance (Chapais, 2004; Thierry et al., 2004).

The second scenario builds on the assumption that both COMT alleles can act as a risk factor in different environmental conditions (Goldman et al., 2005; Weeland et al., 2015). Genotype might modulate individuals' differential susceptibility to contexts (Simons et al., 2011). The favorable social environment for individuals carrying allele $\mathrm{X}$ can be the adverse social environment for individuals carrying allele Y. Taking another step towards speculation in Assamese macaques, an individual's allostatic load might partly depend on its genotype. In this sense, it is probably more stressful for Met-carriers to be in a higher-ranking position, in which they increase their aggression rate, than to be in a lower-ranking position. On the other hand, this would be the other way around in Val-carriers whose adverse social environment would be a low-ranking position in which they express higher aggression rates than in higher-ranking positions. This explanation fits well for both sexes.

However, any further clarification of these scenarios will require future studies with specific research questions on more fine-graded effects of COMT Val ${ }^{158} \mathrm{Met}$. These could include detailed data on coalition formations and relatedness, as females of the same matriline are generally more likely to end up in similar rank positions (Thierry et al., 2004). If this polymorphism is detected also in other macaque species with captive individuals available, further experimental testing of arousal and other responses to different social environmental conditions could also contribute to our understanding of gene-environment interactions between rank and $\mathrm{Val}^{158} \mathrm{Met}$. Such laboratory studies in which dominance ranks can also be experimentally influenced (Kohn et al., 2016; SnyderMackler et al., 2016b, 2016c), could also verify different COMT enzyme activities and investigate how dopamine levels relate to aggression under different conditions.

Study 3 (chapter 4) demonstrated that strong social bonds have a buffering effect on HPA-axis activity, but not in all individuals. Specifically, a main buffering effect was not observed in male Assamese macaques towards the high end of the GRS, who carry the rarer alleles putatively causing amino acid exchanges in HPA-axis genes. The buffering effect of strong social bonds on HPA-axis activity seemed to decrease and even 
disappear when moving from individuals at the low end of GRS to the high end of GRS. Probably, the attenuating effects of social bonds on HPA-axis activity cannot be put into effect due to an altered HPA-axis regulation and the adaptive benefits of social bonds are not observable as a cortisol downregulation in all individuals. However, even at high GRS, male Assamese macaques might still benefit from buffering effects on health symptoms other than HPA-axis activity, as the benefits of social bonds might go beyond that on a physiological level, including cardiovascular, neural and immune functions (Uchino, 2006), and on a behavioral level, including social support in coalitions and access to resources (Sabbatini et al., 2012; Schülke et al., 2010; Tiddi et al., 2011; Young et al., 2014b). In Assamese macaques, the strength of social bonds with adult males predicts agonistic support in immatures, adult females and adult males (Haunhorst et al., 2017; Minge et al., 2016; Schülke et al., 2010). Female Assamese macaques also benefit from strong social bonds with males in the form of increased co-feeding tolerance and food intake rates (Haunhorst et al., 2017). Due to the multidimensionality of the effects of social bonds, more studies investigating the potentially more nuanced benefits on variables decoupled from HPA-axis activity are needed.

As risk-taking behavior declined with increasing GRS, male Assamese macaques probably follow the strategy to refrain from initiating fights, as aggressive interactions are not only costly with regard to injury risk, but also in terms of cortisol elevation causing impaired immune functions (Cole et al., 2009; Elenkov \& Chrousos, 1999; Godbout \& Glaser, 2006; Padgett \& Glaser, 2003; Wittig et al., 2015). Aggressive behavior is linked to cortisol release (Halász et al., 2002; Kruk et al., 2004; Wittig et al., 2015). When already suffering from elevated cortisol levels or an altered HPA-axis regulation, males can probably not downregulate their physiological stress response as efficiently as others. Therefore, these males might attempt to avoid situations likely to increase cortisol levels and thus refrain from initiating aggressive interactions. Dominance rank did not significantly predict behavioral or endocrine variation in any of the analyses, but showed a statistical trend to increase risk-taking. This seems plausible, as the actual risks associated with starting a fight may indeed be lower in higher ranking individuals. To better identify the different strategies of male Assamese macaques, lifetime data on their careers will be needed in the wild. These long-term approaches will allow to investigate whether males with different GRSs have fundamentally different social and reproductive 
strategies and whether mismatches between genotype and reproductive strategy lead to detrimental outcomes.

Probably, low GRS individuals take more risks and initiate fights to attain higher social status while benefitting from social buffering by their close bonds, whereas high GRS individuals are less risk-taking, do not benefit from a social buffering effect on cortisol levels and might rather cue for dominance. The observed phenotype of males with high GRS might thus reflect an alternative strategy in which they constantly keep cortisol levels high and energy mobilized. This rather permanent state of arousal might go hand in hand with increased social maintenance. These male Assamese macaques might be more passive in agonistic behavior and rank attainment but take a more active role in bond maintenance. Alternatively, regarding that at low to no risk in HPA-axis-related genes the residuals of all responses in study 3 varied more widely than at GRS $\geq 10 \%$, it could be hypothesized that male Assamese macaques with low genetic risk are more flexible in their social engagements and thus show more variation in their behavioral strategies than individuals with a higher genetic risk, who are probably more constrained. Lifespan data on male coalitions, reproduction, survival, rank attainment and maintenance will reveal how individual strategies are shaped by genetic predisposition under natural selection pressures and how rare alleles are maintained in the wild.

In Assamese macaques male dominance rank, which is predicted by coalition formation, is positively correlated with reproductive success (Schülke et al., 2010). On the one hand, male Assamese macaques with a high GRS probably rather queue for dominance than to engage in coalitionary aggression to attain a higher rank. On the other hand, there might exist alternative strategies to increase reproductive success besides being active in forming alliances or queuing to rise in rank. As female reproductive synchrony is high and alpha male paternity is relatively low in Assamese macaques (29\% in PKWS, Sukmak et al., 2014a), a high dominance rank might not be the only way to increase reproductive success. Probably, the less risk-taking individuals with a higher GRS invest more in gaining benefits from heterosexual associations. There is support for the "friends with benefits" hypothesis in Assamese macaques, as male-female association during the mating season predicts male mating success (Ostner et al., 2013). The formation of same- and opposite-sex social bonds, low alpha paternity and female reproductive synchrony in this species builds a basis for different male strategies, that 
could have at least partial genetic predisposition. These predictions need further testing in the future, including lifetime social and paternity data of males to decipher potentially different careers.

The findings in this thesis demonstrate that the incorporation of genotype has the potential to elucidate previously not recognizable patterns in social bonds, proximity maintenance and the main social buffering effect in wild male Assamese macaques. Perhaps, the consideration of underlying genotypic effects would also uncover new trends in previous behavioral ecology studies including also non-findings. For example, mateguarding has not been associated with energetic costs in male Assamese macaques (Schülke et al., 2014), but genetic risk could moderate the effects of mate-guarding and correlated variables on GC output. Genotype could also influence within-group spatial position (Heesen et al., 2015), for example if male Assamese macaques are more risktaking with decreasing GRS and thus more likely to end up in rather peripheral positions. Regarding male dominance and aggression, a previous study has demonstrated that GC levels during the mating season are negatively related with dominance rank and positively with the amount of aggression received in adult male Assamese macaques, but not in large subadult males, who are also involved in reproductive behavior (Ostner et al., 2008a). Also, adult males showed significantly higher GC levels than large subadult males during the mating season (Ostner et al., 2008a). Hypothetically, a non-random distribution of functional genetic variants influencing GC levels between the six adult and the six large subadult males included in that study could have caused these findings to be interpreted as an age effect. This line of thought gives another example for the importance of including or at least controlling for genotype.

Regarding the compelling evidence for genotypic contribution to HPA-axis activity and social behavior in humans, rodents and other model organisms (e.g. Anholt \& Mackay, 2012; Ising \& Holsboer, 2006; Maxson et al., 2001; Wasserman et al., 2010), the general patterns detected in this thesis are unlikely to be limited to Assamese macaques. The HPA-axis is an evolutionary conserved pathway which is fundamental for many main body functions in vertebrates (Charmandari et al., 2005; Munck et al., 1984; Schulkin, 2011). With a high probability, polymorphisms in HPA-axis genes can be linked to similar patterns in other macaque species and probably also in other social mammalian taxa. Moreover, social buffering is not limited to mammals and studies suggest a shared 
evolutionary origin in vertebrates (birds: Edgar et al., 2015; fish: Faustino et al., 2017). The impact of genotype on social buffering is likely not unique to Assamese macaques but constitutes a more universal phenomenon repeatedly observable across the animal kingdom. The population of Assamese macaques residing in PKWS were ideal to start studying the genetic influences on social buffering in a free-ranging animal species because males form strong social bonds with each other (Kalbitz et al., 2016) and the adaptive value of these social bonds have been previously demonstrated in this population (Schülke et al., 2010). In addition, samples for molecular analyses could be obtained from individually identifiable animals in the long-term field project. As a next step, similar approaches could be tested in macaque species with different social styles in the other grade classes of macaques (Thierry, 2000; Thierry et al., 2008).

The results in this thesis indicate that variation in the patterns of affiliation and HPA-axis activity might result from variation in genotype. Consequently, social support might not have beneficial effects on well-being in all individuals, which could constitute a relevant informative function in health assessment. To understand how genetic and social factors interact to influence individual factors that contribute to a broad scope of health symptoms is crucial to advance clinical applications (Chen et al., 2011a). The processing of emotions and the susceptibility to disorders are coupled to a variety of neurobiological processes. A thorough investigation of the genetic basis of neurotransmitter variations will help to understand these processes (Hill et al., 2018).

\subsubsection{An evolutionary scenario}

Why do seemingly detrimental alleles still exist within populations? Probably, because they only appear detrimental by first impression, because (i) we miss some aspects of the animals' lives in which certain characteristics would actually be beneficial, (ii) we investigate only a limited time interval with certain physical and social environmental conditions such as levels of predation risk, food availability, stable or instable hierarchies, intra- and interspecific competition. Therefore, the data can reflect only a snapshot of a specific timeframe rather than evolutionary processes. In years, decades, centuries or millennia some of these environmental aspects may change and 
those individuals who seem "genetically vulnerable" at the time point of investigation, might benefit from their phenotypic characteristics under different circumstances. This could work in different ways, for example increased vigilance could help during periods with high predation risk or in the face of high competition (Dobson \& Brent, 2013).

Over evolutionary times the balance of different traits may preserve polymorphisms for different phenotypes in a population. This would cause different strategies for individuals, depending on the underlying genotype. This evolutionary phenomenon is called balancing selection, acting for example when there is environmental heterogeneity over time. As the result of repeated adaptation processes, balancing selection is considered to be the main mechanism maintaining genetic diversity (Charlesworth, 2006; Schaschl et al., 2015). Interestingly, a comparison between three selection scenarios (selective neutrality, mutation-selection balance and balancing selection) that could explain the genetic variation in personality also revealed that balancing selection is the evolutionary genetic mechanisms that explains genetic variance in personality traits best (Penke et al., 2007; Penke \& Jokela, 2016).

In the multi-male, multi-female gregarious Assamese macaque living in seasonal rainforests, fluctuating conditions, including the physical and social environment, such as climate, food availability, predation pressure, intra- and inter-specific competition, population density, group size and composition, might impact reproductive success. Additionally, genotype might play an important mediating role in how individuals react to these changing circumstances. Genetic variation influencing male Assamese macaques' HPA-axis activity might cause periodical changes in reproductive success. This might be caused by differential stress vulnerability, sensitivity to competition (Dobson \& Brent, 2013), the availability of partners to form social bonds with and risk-taking behavior. Risk-taking could be beneficial under circumstances of low or medium predation, low food-availability and female-biased group compositions, whereas risk avoidance and hypervigilance could bear advantages during times of high competition and predation (Dobson \& Brent, 2013). Vice versa for example, risk avoidance such as hypervigilance demands unnecessary energy during times of low or medium predation (Dobson \& Brent, 2013). It is also conceivable that a certain age-dependency moderates the pros and cons of each genotype over the lifetime of Assamese macaques, e.g. factors such as risk-taking and exploring unfamiliar terrain that could influence infant or juvenile mortality 
(although low in Assamese macaques) as well as male dispersal and reproductive activity. In male rhesus macaques, the 5HTTLPR polymorphism in the serotonin transporter gene is related to the timing of natal dispersal and reproductive success (Krawczak et al., 2005; Trefilov et al., 2000). Heterozygous males are more reproductive than homozygous males at an intermediate age, whereas they are less reproductive at a younger and an older age. thereby causing the polymorphism to appear selectively neutral because over the males' lifetimes there is no difference in the total reproductive output (Krawczak et al., 2005). Similar age-dependent influences of HPA-axis gene variants on reproductive success could apply also to male Assamese macaques. If increasing GRS leads to generally less risk-taking behavior, this could lead to later emigration, probably causing them to initiate reproduction already in their natal group. Additionally, future studies could test how genotype influences the frequency and nature (alone or in company) of both natal and secondary dispersal and the integration process after changing into a new social group. A genotype predisposing for risk-taking could be beneficial in terms of initiating social interactions with unfamiliar individuals.

Regarding the differentiated formation of social bonds and individuals' positions within the friendship network, there is preliminary evidence from human studies depicting that genetic make-up, including variation in HPA-axis genes, impacts the clustering or separation of individuals within the social network (Fowler et al., 2009, 2011). These correlated genotypes in social networks indicate that homophily or heterophily obtain on a genetic level (Fowler et al., 2011). This could result in variation in the alliances an Assamese macaque forms depending on e.g. the genotypes, personalities and behavior of the potential partners available in the group, moderated by an individual's own genotype. For example, individuals might prefer to invest in relationships with individuals who have favorable genotypes (Ostner \& Schülke, 2018). In this context, also indirect genetic effects, where genetic variations in conspecific group members influence an individual's phenotype leading to complicated and sometimes non-intuitive evolutionary consequences, can come into play (Montiglio et al., 2013; Moore et al., 1997; Wilson et al., 2009; Wolf et al., 1998). Variation in the resulting coalitions could lead to differences in reproductive success in Assamese macaques (Schülke et al., 2010). Genotype might thus influence reproductive success in intervals in a positive or negative way, balancing out the genotypic benefits and drawbacks over an individual's life time, generations or millennia leading to similar genotype frequencies. 
Balancing selection is also assumed to be the major mechanism maintaining both alleles of COMT Val ${ }^{158} \mathrm{Met}$ in humans (Goldman et al., 2009). The counterbalancing benefits and drawbacks of carrying the Met or the Val allele, including susceptibility to stress, pain and mental disorders (higher in Met), creativity (higher in Met) and cognitive performance (better in Met), are summarized in the warrior-worrier balancing selection model (Goldman et al., 2005; Stein et al., 2006). Regarding heterozygous individuals, both intermediate phenotypes as well as advantages over both other homozygotes have been reported, for e.g. working memory and protection from schizophrenia (Costas et al., 2011; Gosso et al., 2008). Therefore, COMT Val ${ }^{158}$ Met might also be maintained by heterozygote advantage, a sub-type of balancing selection. However, another sub-type, namely negative frequency-dependent selection, has also been proposed to be the evolutionary mechanism behind the maintenance of this common polymorphism (Goldman, 2014). This is also one of the most plausible mechanisms proposed for the maintenance of different personality traits and levels of aggression (Smith et al., 1988; Smith, 1982; Penke et al., 2007). Considering that the environmental conditions of a diverse rainforest are very likely to change over centuries and the fact that the genotype frequencies of COMT $\mathrm{Val}^{158} \mathrm{Met}$ in the Assamese macaque population of this thesis were similar to those observed in human populations, the results might be an indicator that balancing selection is also the main mechanism maintaining the polymorphism in this primate species.

Both direct and interaction effects have been attributed to COMT $\mathrm{Val}^{158} \mathrm{Met}$. Recently, a study on this polymorphism focused on the U-shaped curve of dopamine activity with both low or high levels being maladaptive (Goldman-Rakic et al., 2000; Hill et al., 2018; Vijayraghavan et al., 2007). Hill and colleagues (2018) propose more nuanced relationships for COMT than the dichotomous warrior-worrier model. The maintenance of genetic variation over time is probably not easy to explain and several genotypic effects not easy to detect, because they have a more complex mediating role on phenotypic variation and/or are context-dependent. 


\subsection{Behavioral genetics of the HPA-axis}

\subsubsection{The heterogeneity of defining and measuring HPA-axis activity and social behavior}

The first study on the heritability of hair cortisol was conducted with a non-human primate, the vervet monkey (Fairbanks et al., 2011). A heritability estimate of $30 \%$ was recorded during periods of both high and low environmental stress. A review on the heritability of human cortisol levels reports estimates of up to $84 \%$, with a high variation and a median of $52 \%$ (Bartels et al., 2003). A meta-analysis of comparable studies, however, revealed a heritability of $62 \%$ (Bartels et al., 2003). The $84 \%$ heritability have been reported for cortisol levels in response to a CRH stimulation (Kirschbaum et al., 1992). 40 - $69 \%$ have been reported for salivary cortisol under different conditions (e.g. morning, evening, circadian rhythm, after playing computer games, van Hulle et al., 2012; Riese et al., 2009; Steptoe et al., 2009; Young et al., 2000) and 65 - $72 \%$ for hair cortisol (Rietschel et al., 2017; Tucker-Drob et al., 2017). Another interesting case of heritability of the HPA-axis response was described in an experiment by Federenko et al. (2004). They measured salivary and blood cortisol, ACTH levels and heart rate of twins in response to three repetitions of the Trier Social Stress Test (Kirschbaum et al., 1993). They found that the heritability of all measures was significant and increased from the first exposure to the stress test (heritability of $8-32 \%$ ) to the third (all heritability estimates greater than $97 \%$ ). From these results they concluded context-dependent genetic influences, with higher heritability in low-anxiety, compared to new, anxietyevoking situations.

Working with different model systems, conditions and sampling material is subject to differences in defining and measuring variables. The application of various methods of measurements in human studies, resulted in different effect sizes and variances that can be explained by genotype. In study 3, I was able to demonstrate that a $17 \%$-increase of the GRS was associated with a $20.5 \%$ increase of the response variable immunoreactive urinary cortisol level. Prospectively, further research on this topic might reveal higher or lower effect sizes depending on sampling material (e.g. urine, feces, 
saliva, hair, blood), protocols, time and frequency (Bartels et al., 2003). This thesis contributes to the general understanding of an underlying genetic basis, which can be elaborated on in future studies, e.g. by testing more situation-specific setups.

Differences in the study design and the measurements of aggression have also been discussed as a weakness in meta-analyses on the heritability of aggression, which yield estimates between 40 and $56 \%$ in humans (Ferguson, 2010; Miles \& Carey, 1997; Rhee \& Waldman, 2002). Issues might emerge from the inconsistencies of the applied questionnaires and a neglect of the difference between aggression and antisocial behavior (Qayyum et al., 2015). Antisocial behavior is by definition always aggressive, but not vice versa. To classify as an antisocial behavior, an action must be out of proportion to a complex or provoking stimulus and the affected individuals must suffer from a disadvantage due to a violation of social norms (Baron \& Richardson, 1994; Ferguson, 2010; Qayyum et al., 2015). Further, it has been criticized that aggression is treated as a single behavior (e.g. Wrangham, 2018). Aggression can be divided into several subclasses, with reactive and proactive aggression being a commonly used distinction. Proactive aggression refers to a planned attack with a purpose to reach some kind of goal, whereas reactive aggression is an reaction to a frustrating or threatening event (Wrangham, 2018). The division between proactive and reactive aggression is supported by data indicating different neural bases between those two aggression subtypes (Dambacher et al., 2014, 2015). A moderate heritability for both types of aggression has been reported, for example 27 - $42 \%$ for reactive and 39 - $45 \%$ for proactive aggression in humans (Paquin et al., 2014). Another longitudinal study found a $48 \%$ estimate for reactive and a $85 \%$ estimate for proactive aggression (Tuvblad et al., 2009). In nonhuman primates it is assumed that proactive (e.g. infanticide) is rare compared to reactive aggression (e.g. most agonistic interactions that arise for example from mate or food competition, Lukas \& Huchard, 2014; Wrangham, 2018). A recent study, however, demonstrated the importance of social environment, e.g. rank and sex, for the propensity of Barbary macaques to employ reactive or proactive aggression (Paschek et al., in press). If of interest, the distinction between reactive and proactive aggression could also be emphasized in future studies on the genetic foundations of aggression in wild macaques.

A split picture for the effects on phenotypic variation also emerged for the human COMT $\mathrm{Val}^{158} \mathrm{Met}$ polymorphism. On the one hand, some of the associations have been 
replicated (e.g. Bouma et al., 2012; Quednow et al., 2009; Strous et al., 2003) and two meta-analyses found an overall effect of COMT $\mathrm{Val}^{158} \mathrm{Met}$ on schizophrenia and suicide (Bhakta et al., 2012; Kia-Keating et al., 2007; Singh et al., 2012). In a neurological metaanalysis COMT Val ${ }^{158}$ Met explained $12 \%$ of the variance in tests of prefrontal activation (Mier et al., 2010). In response to adverse stimuli, COMT Val ${ }^{158}$ Met explains $13-38 \%$ of the variation of prefrontal cortex reactivity as a single polymorphism (Drabant et al., 2006; Smolka et al., 2005). Considering the usually rather small effect sizes for single loci, COMT Val ${ }^{158} \mathrm{Met}$ appears to play an exceptional role in the emergence of certain phenotypic characteristics. On the other hand, the SNP did not withstand a meta-analysis on violent and aggressive behavior (Vassos et al., 2014). In a recent review on aggression and COMT Val ${ }^{158}$ Met, Qayyum et al. (2015) point out that inconsistent findings and meta-analyses are not surprising considering the differences in assessing aggression as well as differing age and sex ratios of participants in these studies.

In addition to cortisol and aggression, genetic influences on personality and social behavior have also gained attention in behavioral genetics and approximately $50 \%$ of the variation of certain traits are explained by genotype (Barban et al., 2016; Ebstein et al., 2010; Ferguson, 2010; Mills \& Tropf, 2015; Plomin et al., 2016; Robinson et al., 2008). For example $56 \%$ of human antisocial personality and behavior can be explained by genetic influences (Ferguson, 2010). Further, most aspects of relationships between parents and offspring, siblings, friends and spouses underlie substantial genetic influences (Plomin, 1994; Plomin et al., 2013; Ulbricht \& Neiderhiser, 2009). This also holds true for the types of friends individuals choose (Beaver et al., 2009; Iervolino et al., 2002; Plomin et al., 2013). Notably, these findings on family, peer and romantic relationships are mainly based on self-, parent- and/or peer-reports and questionnaires designed to measure specific relationship aspects such as satisfaction with the relationship, closeness, passion, relationship quality, habits of their friends, attachment, involvement and peer preferences. Regarding the genetic underpinnings of sociality, social science has mainly focused on factors in criminology, demography, education, intelligence and topics in social psychology. The influence of genotype on day-to-day social interactions is not well explored, neither in humans nor in non-human primates. In humans social bonds are measured mainly on an emotional and not a behavioral level. Besides questionnaire-based studies, some cases applied social network analysis and reported correlated genotypes in friendship networks (Fowler et al., 2011) and genotypic influences on social network 
positions in social and agonistic networks of humans and macaques (Brent et al., 2013; Fowler et al., 2009; Lea et al., 2010). Research on sociality in laboratory rodent models is mostly limited to quick experimental assays like test batteries (Kaidanovich-Beilin et al., 2011; Silverman et al., 2010; but see König et al., 2015). In wild primate studies measures are not generated from ratings or questionnaires but derive from direct observation of predefined naturally occurring behaviors in the group.

Such direct measurements of HPA-axis activity and aggression were applied in the studies 2 and 3 (chapter 3 and 4). In this thesis, the cortisol levels reflect the natural range and not responses to artificial tests or hormone administrations. Social behavior was recorded based on standardized protocols and pre-defined variables from an ethogram. The results demonstrate the effects of genotype on naturally occurring HPAaxis activity behavioral variation.

The mentioned differences between studies, including non-standardized age and context-control, might explain the mixed findings in behavioral genetic approaches. This applies to heritability measures as well as to single and multi-locus approaches. Regarding the frequent behavioral genetics approach to investigate the heritability of traits in humans, it must be pointed out that it has demonstrated the importance of genetic impacts, but it allows only to identify a general genetic basis, and not which genes are involved in certain traits in particular. Of the numerous polymorphisms occurring across the genome, the majority is benign, some are advantageous, protecting individuals under adverse conditions, and some are malign because they directly impair phenotypic traits, cause diseases or increase the susceptibility for certain conditions (Manolio et al., 2009; Manzoni et al., 2018; Williams, 2006). It has been a longstanding aim to decipher the sets of genes that are involved in the emergence of complex phenotypic traits like stress resilience and social behavior. This thesis contributes to that by identifying new putatively functional polymorphisms in HPA-axis genes and associating them with cortisol levels, aggression rates, social bonds as well as their maintenance and buffering effects. However, especially candidate gene associations rely heavily on repeated associations, which is why the results presented here should be confirmed by replication. Regarding the direct measures resulting from quantification of behavior and hormones in non-human primate studies, replication studies could be conducted relatively easy and be a major contribution to a clearer picture of genotype-phenotype associations. When 
behavioral genetics approaches increase in wild primate studies, this might help to forestall a replication crisis. With respect to the significant impact of the COMT $\mathrm{Val}^{158} \mathrm{Met}$ polymorphism, its detection and linkage to a similar phenotype in a non-human primate (chapter 3), this thesis represents an important step towards understanding individual variation in aggression and may substantiate future studies on the evolutionary roots of inter-individual variation in human aggression.

\subsubsection{The effects of social bonds}

Besides the issues with defining and measuring aggression and HPA-axis activity (chapter 5.4.1), there also exist differences in how social bonds and social buffering are measured. Already in the early days of the social buffering hypothesis, Cohen and McKay (1984) pointed out that despite the large body of research on the social buffering hypothesis, the results are inconclusive, probably due to methodological inconsistencies. In the literature on social support and health, important sources of the mixed findings are also the measurement and conceptualization of social support (Uchino et al., 1996). These measures range from being structural (e.g. network analyses) to functional (e.g. emotional reporting, Uchino et al., 1996), can be direct or indirect (Ostner \& Schülke, 2018) and include perceived or real support (Holt-Lunstad et al., 2010).

In captivity, studies can investigate interaction effects and often focus on the negative impacts of social isolation by stressing individuals with their close social partners being present or absent (Cacioppo et al., 2015; Cole et al., 2015; Hawkley et al., 2012). In the wild, studies primarily focus on the positive impacts of the presence of social partners (Wittig et al., 2016; Young et al., 2014a) and socio-positive behavior as a mechanism to cope with stressors (Cheney \& Seyfarth, 2009; Crockford et al., 2008; Wittig et al., 2008). In this thesis, I investigated genetic contribution to the main buffering effect with regard to male-male social bond strengths and average cortisol levels reflecting everyday stressors in a wild primate population. No interaction effect was tested because it was not feasible to track variation in a relevant stressor and the HPAaxis response on a fine-enough temporal scale. This would be, however, possible under experimental conditions or in other species in which endocrine samples can be assigned 
to single stressful events (e.g. Preis et al., 2018; Wittig et al., 2015). The combination of using objective measures of social behavior in wild animal studies, like proximity, body contact and grooming in the CSI, and the application of genotyping might help to clarify the inconsistencies in the current literature and extend our knowledge on mechanisms behind social phenomena like the formation of long-lasting strong social bonds and social buffering.

Currently, it is generally assumed that having social bonds can reduce stress and promote health (Hennessy et al., 2009). Early studies on the link between sociality and fitness in female baboons, demonstrated that social bonds enhance longevity and offspring survival (Archie et al., 2014; McFarland et al., 2017; Silk et al., 2003, 2009, 2010b). Up to now, the mechanisms linking sociality to fitness are not yet completely understood, but partly this phenomenon has been explained by agonistic support assisting in rank acquisition and protection from harassment, social buffering and mitigated physiological stress, increased access to resources, reduction of predation risk and thermoregulation (Campbell et al., 2018; Ostner \& Schülke, 2018). Thermoregulative effects include lower energy expenditure in wild Barbary macaques with more social partners that form larger huddles (Campbell et al., 2018), higher homeothermy and lower hypothermy in vervet monkey with a greater number of social partners (McFarland et al., 2015), increasing stress-alleviating effects of the strongest social bond with decreasing daily temperatures in wild male Barbary macaques (Young et al., 2014a) or greater survival of individuals with many grooming partners in cold winters (Lehmann et al., 2016).

Hitherto, it has been assumed that strong social bonds have positive effects on fitness, but recent findings suggest that strong social bonds do not necessarily increase fitness in all gregarious primates and can even be costly under certain circumstances (Thompson \& Cords, 2018). Therefore, the effects of social bonds are probably not as simple and unidirectional as previously assumed. In humans, the effects of social relationships might be additionally mediated by negative feelings like anxiety, loneliness and depression (Lee \& Robbins, 1998, 2000; Williams \& Galliher, 2006). Some aspects of social relationships might even be sources of stress, which balance out the ameliorative effects of social support (Thoits, 2011). In a study on blue monkeys (Cercopithecus mitis stuhlmanni), Thompson and Cords (2018) argue that the effects of social bonds on 
longevity are context-dependent and can even lead to increased mortality risk under certain circumstances in this species. This happens to be the case when top partners change and are not consistent over time (Thompson \& Cords, 2018). Seyfarth et al. (2012) also speculated that a generally high social activity without a strong discrimination might carries costs for female baboons (Papio hamadryas ursinus). In capuchins (Cebus capucinus imitator), infants of highly social females are at a greater risk of dying during alpha male rank changes than infants of less social females, whereas the offspring of highly social females has a higher survival probability during stable periods (Kalbitzer et al., 2017). Further, the findings of a recent study on female baboons (Papio cynocephalus) challenge the causal connections between sociality and longevity (Tung et al., 2016). In their study, Tung and colleagues (2016) demonstrate that adversity during early lifetime predicts both later social integration and longevity. Therefore, social bonds might not increase longevity directly, but rather could be a signal of a common underlying driver of sociality and longevity. Besides such early environmental influences on later sociality, additional mechanisms, like underlying genetic constitution, might also impact the patterns of social bond formation. In the future we need to determine whether genetic make-up acts similarly in affecting both adult sociality and fitness outcomes to generate a pseudo-correlation between the two variables. The results of this thesis contribute to our understanding that a significant proportion of sociality is probably the outcome of DNA variation, something existent in individuals even before the actual formation of social bonds. In this sense individuals might match their social activities to their genotype.

To my knowledge, this is the first study to look at the effects of social bonds in a behavioral genetics approach in wild non-human primates. Social support is a multidimensional construct (Uchino et al., 1996) and the results of this thesis also support the notion that examining the impacts on different levels is seminal for a further clarification in this research field. Probably, an individual's genetic constitution influences the effectiveness of strong social bonds as a buffer against adverse effects of stress. Furthermore, genotype might influence which benefits of social bonds can or cannot come into effect. Physiologically, cortisol levels might not be ameliorated, whereas other potential bonding effects on adrenaline, oxytocin or arginine vasopressin (Crockford et al., 2017; Kikusui et al., 2006; Koolhaas et al., 2017; Takahashi et al., 2015) might not be altered. Genetic aspects are commonly not a part of reviews on social 
buffering (e.g. Hennessy et al., 2009; Kikusui et al., 2006; Kiyokawa \& Hennessy, 2018), but this should change in the future and genotype mentioned as a potential source of variation. 


\subsection{Mammalian model organisms for the study of behavioral genetics}

\subsubsection{From mice to men}

The mouse is probably the main mammalian model organisms and a prototype of behavioral genetics (Lindzey \& Thiessen, 1970). In particular, mice are highly valued for helping to identify genes and polymorphisms as well as understanding their functionality (Plomin et al., 2013). Studies on COMT and MAOA knockout mice were conducted following first evidence for a functionality from variation in these genes in humans (Cases et al., 1995; Gogos et al., 1998). Besides target knockout mice, studies on inbred strains are also common. Thousands of mice strains are available to researchers, who can choose between different databases to find strains with requested genotypes and phenotypic differences in e.g. anxiety, cognition and stress-reactivity (e.g. Mouse Phenome Database, Flint, 2011; International Mouse Strain Resource, Eppig et al., 2015). Recent technological advances like the gene-editing tool CRISPR are promising that in the future genotype modifications will be realized in living organisms (Hess et al., 2017; Murugan et al., 2017; Sternberg \& Doudna, 2015). While the standardized studies on genotyped mice of course offer an immense research potential, their application is usually restricted to laboratory-reared, experimental conditions and invasive methods, and only partly help to understand genotypic effects on naturally occurring behaviors in the wild.

Besides mice, humans are among the best-studied vertebrate species in behavioral genetics. The human and the mouse genome are of similar size: $3500 \mathrm{MB}$, which would equal 3000 books of 500 pages each (Plomin et al., 2013). Both were sequenced around the turn of the century (2001 and 2002, Chinwalla et al., 2002; International Human Genome Sequencing Consortium, 2001; Venter et al., 2001) and around $90 \%$ of the mouse genes have orthologues in the human genome (Gibbs et al., 2004). However, the mouse fails to mimic several aspects of primate - including human - physiology and behavior like immunity, metabolism, reproduction and complex sociality (Elsea \& Lucas, 2002; Ezran et al., 2017). 
Mice are investigated under laboratory conditions. Human subjects live in their present-day natural habitat, but the measures entering the statistical models are usually the results of experimental tests, ratings and questionnaires and not objective quantitative measures of how individuals really behave in their daily live. Human behavioral genetics is particularly sophisticated in research on the general heritability of traits, but there are still many unresolved issues concerning which gene variants determine phenotypic variation in which way. In humans it is difficult to get at daily face-to-face interactions and social relationships are additionally linked to emotion and culture (Fletcher et al., 2015). The ability to measure the occurrence of natural behavior in everyday life is one of the outstanding characteristics of primatology. Long-term field studies on habituated primates that can be individually identified facilitate data collection to combine phenotypic and genotypic variation. Oddly, in non-human primates it is seemingly better investigated how behavior predicts gene structure than how genetic variation predicts behavior (e.g. Altmann et al., 1996; Kalbitzer et al., 2016; Kopp et al., 2015).

As we learn more about behavioral genetics in natural animal populations, we are also learning more about shared biological processes with humans and ancestral and derived characters of human traits. Looking at the phylogenetic tree, numerous species exist between mice and humans, but have not received much attention in behavioral genetics yet. There appears a huge gap of targeted and well-studied species. Even our closest relatives, the non-human primates have been relatively understudied in this field. With this dissertation, I started to partly close this gap by introducing the application of behavioral genetics to wild groups of macaques expressing natural behavior in their undisturbed habitat. I provide evidence for the genetic basis of natural primate behavior and hormone levels, which is essential to understand the emergence of individual phenotypic patterns.

\subsubsection{The genus Macaca in the lab and the wild}

Macaques share similarities in their development, immunology, neuroanatomy, behavior and genes with humans and have the potential to be superior animal models 
(Geyer et al., 2000; Gibbs et al., 2007; Kalin et al., 2007). The study of non-human primates, especially macaques, is an important aspect of the broader field of behavioral genetics, but has been particularly highlighted with regard to stress-related research (Ferguson et al., 2012; Meyer \& Hamel, 2014; Phillips et al., 2014; Vallender \& Miller, 2013). The macaque genome includes polymorphisms orthologous to the human gene variants implicated in HPA-axis regulation, including variants in COMT (chapter 3), MAOA (Newman et al., 2005; Wendland et al., 2006), OPRMI (Miller et al., 2004) and SLC6A4 (Bennett et al., 2002). However, most trans-species polymorphisms between humans and non-human primates have been detected in immunological genes (Cagliani et al., 2008, 2010, 2012). The number of detected trans-species polymorphisms across nonhuman primate species is higher, probably due to less clear taxonomic demarcations (e.g. Macaca: Higashino et al., 2012; Li et al., 2009; Satkoski-Trask et al., 2013; Street et al., 2007; Papio: Charpentier et al., 2012; Keller et al., 2010; Zinner et al., 2013).

So far, the COMT Val ${ }^{158}$ Met polymorphism has not been regarded as a transspecies polymorphism, but as a unique feature of humans (Palmatier et al., 1999; Chen et al., 2004), although investigating only one to two individuals per species cannot exclude the existence of a polymorphism. This thesis now demonstrates that in fact the SNP is not unique to humans, but also present in Assamese macaques, which is suggestive of the presence of the polymorphism in further macaque species. How variations in individual genes in macaques mimic that seen in humans advance the utility of macaques as model organisms in human-related research (Vallender \& Miller, 2013). Future studies investigating this locus in several other species will contribute to a broader understanding of the taxonomic distribution of this polymorphism and give insights about the evolutionary roots of this genetic variation.

Investigating more than one HPA-axis-related locus in parallel, a previous study gave important first insights about genetic predispositions of HPA-axis dysregulation by showing that certain risk genotypes explain the extreme values of adrenocorticotropic hormone suppression in response to dexamethasone administration in captive male rhesus macaques (Ferguson et al., 2012). In addition, this thesis extends the current knowledge by (i) investigating the natural variation of cortisol instead of responses to a dexamethasone suppression test, (ii) demonstrating genetic predisposition in a wild population with a natural combination of individual genotypes, (iii) including all 
individuals, not just those at the extreme ends of the hormone range, (iv) adding the investigation of risk-taking, social buffering and proximity maintenance. To get at cortisol levels typically experienced by individuals when faced with everyday stressors, we collected and averaged several urinary cortisol samples. By including all individuals and not only the extremes we covered the full breadth of naturally occurring phenotypes and demonstrate that genotype contributes also to normal variation and not only abnormalities or extremes (Plomin et al., 2013). Nevertheless, compared to laboratory colonies our study has limitations due to smaller sample size and, as discussed before, the potential confounding variable of relatedness.

The investigation of wild primates, particularly in long-term field projects, holds the potential to expand the knowledge on their natural ecology and behavior and to simultaneously gain new insights into the evolutionary history of humans, current differences and similarities with non-human primates. This can help for example to elucidate the evolutionary origins of technical skills, tool use, culture, complex vocalization, stress-related drawbacks or benefits, the nature of long-lasting social relationships, the necessity of close bonds and other aspects of human social evolution (e.g. Byrne, 2007; Haunhorst, 2017; de Waal, 2009). In all probability, the here reported effects are not unique to Assamese macaques but apply to macaques in general. Going one step further, similar patterns could probably be detected in many other mammalian species living in social groups.

I expect the results in this thesis, together with continuative future work, to contribute to a reinforcement of the macaque animal model in behavioral genetics. In this respect, data collection from long-term field projects, which exist for different macaque species, will provide insights into the genetic foundations of the natural behavioral range and physiological phenomena like social buffering. Regarding the linkage between neuropsychiatric diseases and HPA-axis dysfunction in humans (e.g. alcoholism, major depressive disorder, posttraumatic stress disorder, schizophrenia, Carroll et al., 1976; Goeders, 2003; Goodyer et al., 2009; de Kloet et al., 2005; Walker et al., 2008; Yehuda, 1997), the study to understand these traits will require standardized environmental conditions with the possibility to investigate responses to fine-tuned changes. Therefore, research on non-human primates in captivity, in order to understand the development of disorders (e.g. alcoholism in rhesus macaques, Phillips et al., 2014; Schwandt et al., 
2010), is also necessary, besides the investigation of naturally occurring, wild populations. 


\subsection{Conclusions and future directions}

This thesis advanced the current state of the art by (i) introducing a novel genotyping panel useful for the investigation of stress and aggression, (ii) demonstrating that one of the most widely studied human polymorphisms also exists and has similar effects in other species, (iii) responding to recent requests and assessing multiple genes known to affect HPA dysregulation simultaneously instead of the standard procedure of only one or few, (iv) linking HPA-axis-related genetic variation to natural cortisol levels and behavioral patterns instead of psychological disorders or questionnaire-based studies in the case of humans, (v) providing first insights into the genetic influences on social buffering effects in non-human primates and (vi) narrowing the research gap between mice and humans in behavioral genetics and physiogenetics.

Integrating state-of-the-art, non-invasive, molecular methods in research on interindividual differences in a wild primate population, I took the consequent next step following the current standard of knowledge to provide new perspectives on the connections between HPA-axis genotype and phenotype in field studies. The preceding chapters demonstrate that genetic factors account for sizable proportions of individual variation. By considering the influence of genetic variation on physiology and behavior in wild animal populations, we can begin to close important gaps in our current understanding of phenotypic variation. For example, variants in the genetic constitution of macaques may allow individuals to realize different behavioral strategies depending on the context. Certain allelic combinations may provide protection against, or increase sensitivity to stress (Craig, 2007). Genotype is a potent contributor to phenotypic heterogeneity. It is of interest to figure out how and how much of individual variation is explained by variation in gene loci, even if this information is further only used to estimate which explanatory proportion we are missing when ignoring it, or rather how much of the variation is left to be explained by environmental factors.

Finally, I want to give some ideas for prospective studies by which future research could contribute to our current understanding.

First, it is necessary to additionally acquire kinship data to investigate whether the detected genotype-phenotype associations, particularly those in in study 3 (chapter 4), 
withstand controlling for relatedness. As outlined before in the limitations section, accounting for population structure will allow for a more valid interpretation of the data. It is possible that including kinship relations could devour the variation in the GRS, as related individuals may share the same variants in HPA-axis genes. Therefore, the next intended step is to get at dyadic relatedness data and reanalyze the genotype-phenotype associations. Microsatellite markers are currently being analyzed in the Assamese macaques of PKWS and will allow to control for relatedness in the near future.

Second, I would suggest applying the genotyping panel to another species. The next step for this approach would probably be the application to another macaque species. The panel could be applied to macaque species from the other phylogenetical species groups and to more egalitarian and despotic species to detect possible differences in HPA-axis genes between them and compare it with behavioral traits. This might shed light on the underlying causes of differences in social styles in macaques. Further, the panel could also be applied to the sister clade of the macaques, the baboons, and later also to other taxa. Some DNA bases might differ, but mismatches can easily be checked for and new primers designed rapidly. Considering that the genotyping panel was designed for rhesus macaques, but worked for the Assamese macaque study species, it is very likely that the panel is also applicable to other macaque species in its present form.

Third, the approach could also be extended to fully include female and juvenile individuals. Age and sex effects can play important roles in behavioral genetics, behavioral biology and endocrinology including HPA-axis activity (Herman et al., 2016; Kudielka et al., 2004, 2005; Mitchell, 1979; Perry, 2013; Seeman et al., 2001; van der Voorn et al., 2017). In Assamese macaques, for example, females express lower rates of aggression than males (Gutleb et al., 2018b, chapter 3) and large subadult males have lower GC levels than adult males during the mating season (Ostner et al., 2008a). It will be interesting to decipher the impact of biologically relevant age classes with distinct changes in physiology and behavior on the correlation between genotypic and phenotypic variation. In humans some traits like personality and aggression are stronger correlated with genotype during younger age, while individuals sharing genotypic variants become more similar to each other in e.g. their cognitive abilities at an older age (Ferguson, 2010; Plomin et al., 2013, 2016; Plomin \& Deary, 2015; Tucker-Drob et al., 2013). The incorporation of age might also aid in better understanding the social buffering 
phenomenon, as age-specific changes in the ability of female opposite-sex conspecifics to ameliorate HPA-axis responses during stress exposure have been reported (e.g. in guinea pigs, Hennessy et al., 2006). Regarding that many of the behavioral genetics findings have been specific to a certain sex and age class, their consideration in future studies might help to generate more standardized and replicable results and to decipher which genotypic effects are generalizable and which ones are sex- or age-specific.

Fourth, the genotype-related investigation of the social buffering phenomenon could be extended by investigating also the interaction effect hypothesis, which predicts that social bonds have attenuating effects on HPA-axis activity only during stressful events. This thesis focused exclusively on the social buffering main effect, which predicts that social bonds have a general attenuating effect on HPA-axis activity. The genetic contribution to the interaction effect could be investigated with another study design using acute stressors. Probably, another study species which urinates more often and where single stressful interactions can be assigned to certain urine samples, e.g. as it is the case in chimpanzees (Preis et al., 2018; Wittig et al., 2015), could be used for this approach. There is evidence for both the main and the interaction effect hypotheses and they do not have to be mutually exclusive (Cohen \& Wills, 1985). Probably, each effect underlies a different mechanism and by more fine-graded experiments we could extend our knowledge on what the general benefits of social bonds are and which benefits come into complete effect only during stress.

Fifth, an individual's genetic constitution probably influences with whom it has strong social bonds and its responsibility for the maintenance of these bonds. With this respect, an individual's social relationships might correlate with its genotype. An important factor for the maintenance of strong relationships is time investment (Dunbar, 2018; Roberts, 2010; Roberts et al., 2009). Individuals have only a certain time budget which they can allocate to affiliative behaviors. Therefore, individuals are probably limited in how many strong relationships they can maintain. Regarding the constraints in how an animal can allocate its time to different activities, the question arises in whom individuals invest their social time and with whom they form social bonds with. Kinship, age and rank relationships do not explain all of the observed variance in bonding patterns. So far, genotype data has mainly been included to investigate effects of relatedness on bond strength. The consideration of genotype in the study of social relationships might 
reveal alternative scenarios to explain social data (Reiss, 2010), but how wild primates' genotypes contribute to partner relationship and partner choice in a broader sense is not known. In this regard it will be interesting to investigate possible moderating effects of HPA-axis genotype on the formation of social bonds taking into account the available partners and partner features such as genotype and dominance rank. Probably closeness in the social network (Fowler et al., 2011) or friendly behavior towards higher ranking individuals (Seyfarth et al., 2012) reflect specific preferences or social strategies. With the time constraint in mind, the investigation with whom individuals spent time with and if this can be explained by an underlying genetic component will reveal new insights into the mechanisms behind social bonding. Investigating this in the wild will constitute a challenge due to the necessity of large amounts of long-term data reflecting different environmental conditions and it is most likely more feasible to elaborate on these hypotheses under experimental conditions manipulating individual's social environment (e.g. Snyder-Mackler et al., 2016b; Tung et al., 2012).

Sixth, personality could be assessed additionally for the species of interest to investigate how personality and genotype can explain individual variation in combination or in isolation. In conjunction, personality and genotype might explain a larger proportion of the individual variation. Genotype could also be a main predictor of personality and lead to different personality types directly, or by interaction with experience in times of personality development. An HPA-axis candidate locus in AVPRIA has been associated with personality in chimpanzees (Wilson et al., 2017) and a recent study including loci in AVPRIA, OPRM1 and SLC6A3 in captive common marmosets (Callithrix jacchus) found associations with hair cortisol levels and personality domains, but calculated associations for each SNP separately (Inoue-Murayama et al., 2018). The application of an integrated multi-locus approach might therefore also be promising to gain more insights into the relationships between genes, personality as well as behavioral and endocrine phenotypes.

Seventh, long-term data are good, life-time data are better. Coming back to classical primatology studies on social bonds, survival, fitness and longevity (e.g. Cheney et al., 2016; Schülke et al., 2010; Silk et al., 2010b; Tung et al., 2016) individual life-time data could be combined with genotype and social data. Variation in an HPA-axis gene (DRD4) has been associated with longevity in mice and humans (Grady et al., 2013). The DRD4 7R allele is cumulated in humans aging 90 or older. In accordance, DRD4 
knockout mice have a $7-9.7 \%$ decreased lifespan. It is assumed that the effect is mediated by $D R D 4$ affecting responses to the environment and health-related habits like physical activity, thereby indirectly affecting longevity (Grady et al., 2013). This could also be true for social bonds, if being an outcome of underlying genotype, then influencing fitness and/or longevity indirectly. Lifespan data on male reproduction, survival, coalitions, rank attainment and maintenance will reveal how individual strategies are shaped by genetic predisposition under natural selection pressures and how rare alleles are maintained in the wild.

Eight, other pathways than the HPA-axis could be set in the focus of research. Depending on the phenotypes of interest, which could range from infant behavior and maternal care to health markers and aging, corresponding genes could be scrutinized. The genotyping panel in this thesis could be extended accordingly or completely new panels could be designed. For example, opioids might also play a role in social buffering (Kikusui et al., 2006) and more genes coding for respective receptors and the endogenous peptides themselves could be added to the genotyping panel. The integration of more and more gene loci could lead to a better predictability of individuals' phenotypic aspects. Regarding novelty seeking, maternal care and the formation of social relationships, opioid, oxytocin and dopaminergic pathways are highly interesting (Higham et al., 2011; Pearce et al., 2017). These pathways are also promising due to the conservation of the neuropeptide system in vertebrate sociality (Donaldson \& Young, 2008).

My ninth and last suggestion is probably the easiest to conduct: replicate! Unlike in many other research disciplines, replication studies are highly valued and well publishable in the field of behavioral genetics. Thus, the results from this thesis could be replicated in the future to investigate how stable the results are. In a few years' time, new macaques will grow adult and some of the physical and social environmental conditions might have changed or stayed the same. Reproduced results will help to make clearer statements about stable effect sizes and the implications for future studies.

It would also be interesting to look at long-term cortisol in hair samples and validate the functionality of the COMT $\mathrm{Val}^{158} \mathrm{Met}$ and other polymorphisms. Considering the difficulties in hair sample collection, validation and that there is currently no knowledge about hair growth patterns in Assamese macaques, and the lack of sufficient captive Assamese macaques from whom invasive samples from all genotypes could be 
collected, there is still some way to go before these approaches can be implemented. In this sense, working with wild animal populations involves several constraints, but offers the possibility to gain a comprehensive understanding of naturally occurring genotypic and phenotypic patterns.

As a closing remark, I would like to emphasize that often genetic and socioecological approaches both have a shared objective: the explanation of individual variation. The debate about nature or nurture is over, today we know that genes and environment act in concert to influence individual variation, while the respective impacts vary across traits. Studies in behavioral ecology can make use of Tinbergen's four levels of analysis (ultimate: adaptation and evolution, proximate: causation and development, Tinbergen, 1963) to gain an integrative understanding of behavior. Future research is best advised to include the complementary proximate and ultimate categories to explain larger proportions of individual variation and elucidate previously unidentified patterns in social phenomena and strategies. To say it with the words of Jeffrey Rogers: "My own view is that the field of NHP [non-human primate] behavioral genetics is a vibrant and productive aspect of primatology, and that it has outstanding potential for significant discoveries and advances in the near future" (Rogers, 2018, p. 33). 


\section{Supplementary material}

\section{Study 1}

(a)

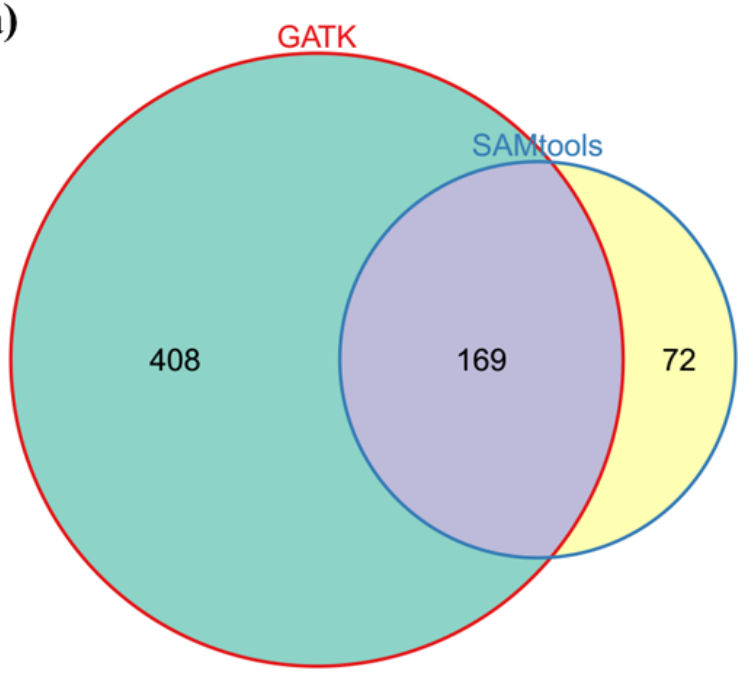

(c)

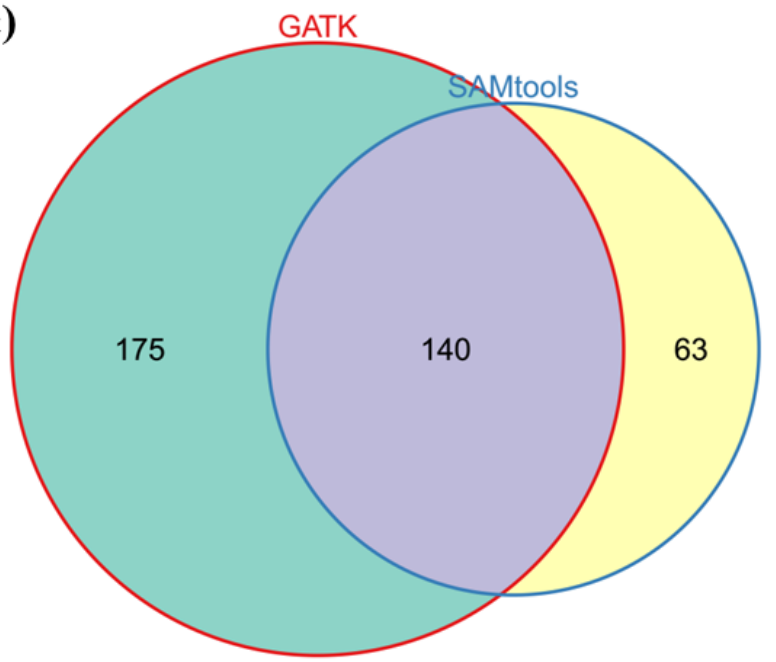

(b)

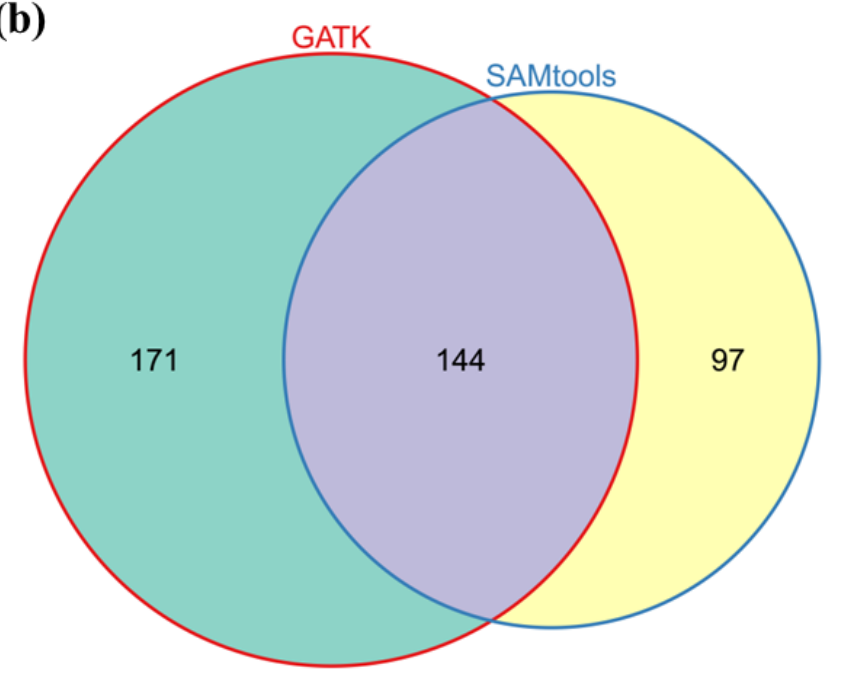

(d)

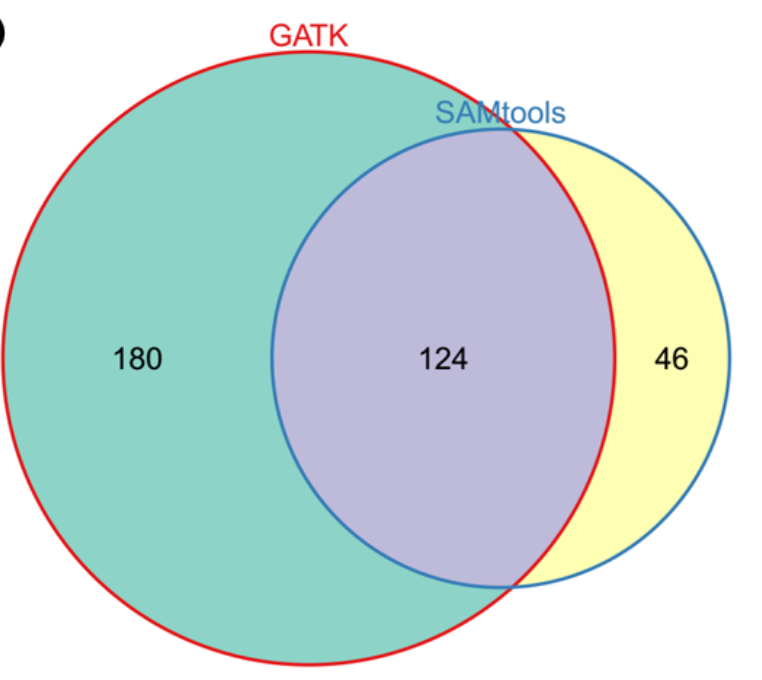

Figure S1.1: Sum and overlap of called variants using GATK and SAMtools pipeline (a) without any filtering, (b) with GATK-filtering, (c) with GATK-filtering and Phred score (QUAL) quality filter $\geq 30$, (d) with GATK-filtering and QUAL $\geq 100$. 


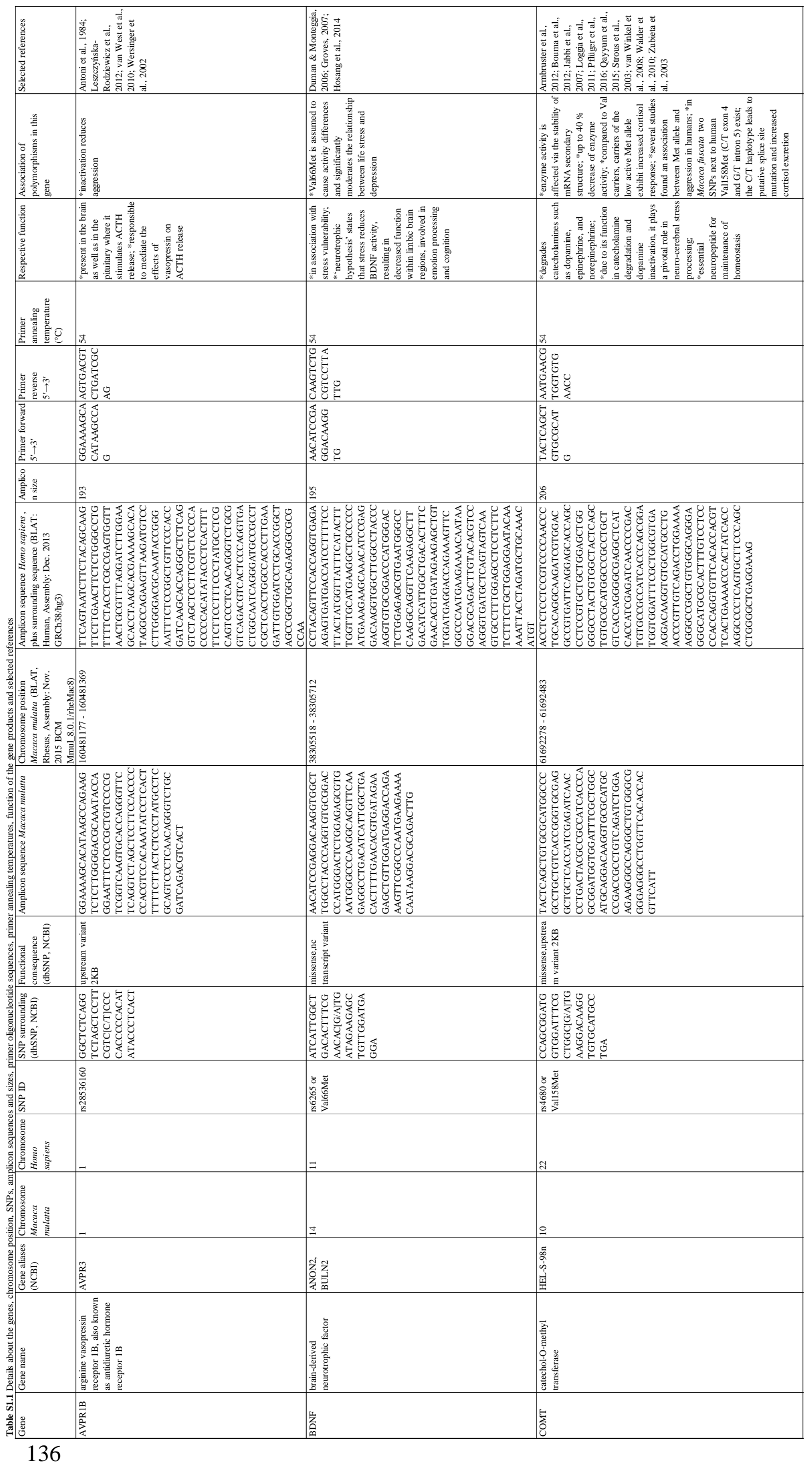




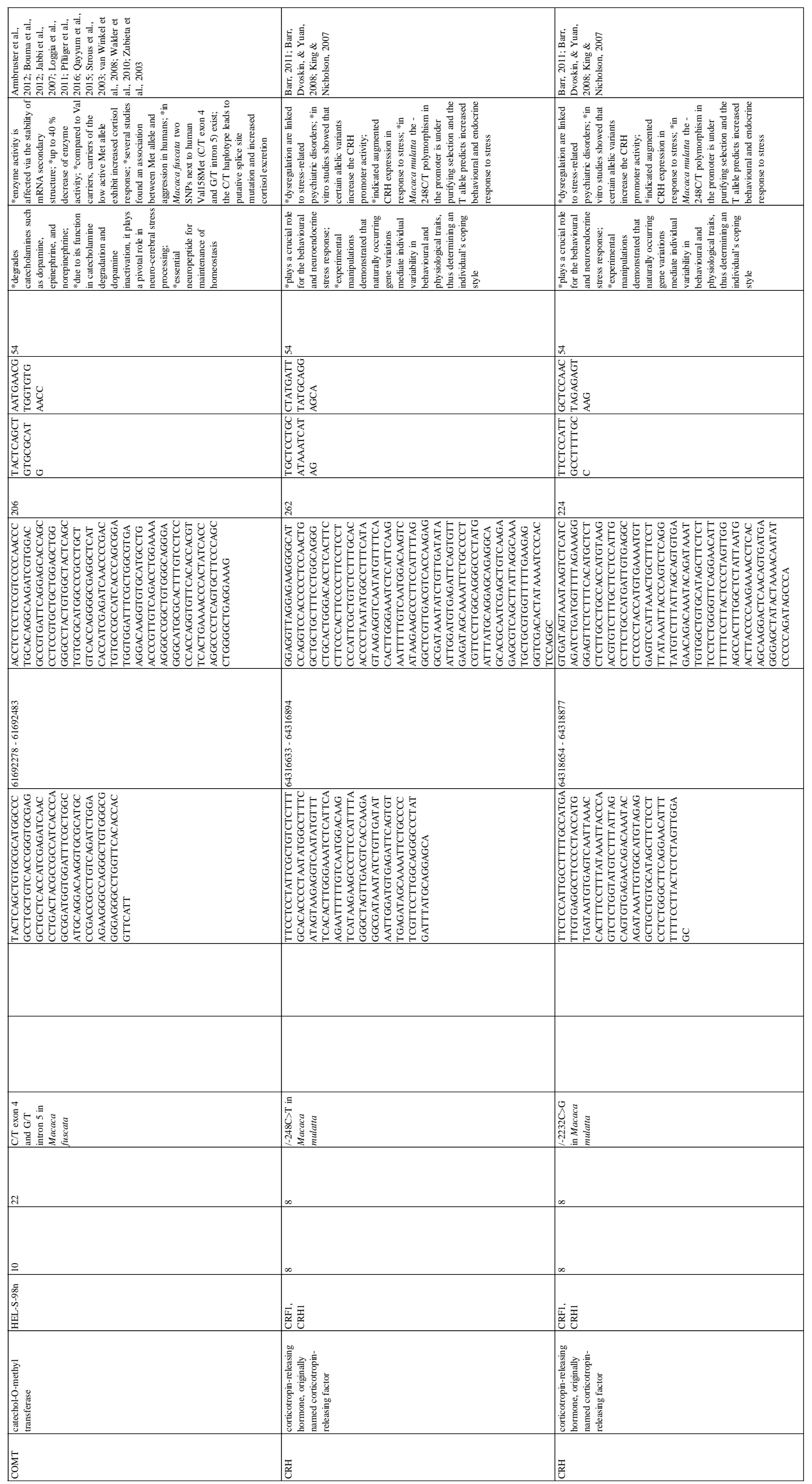




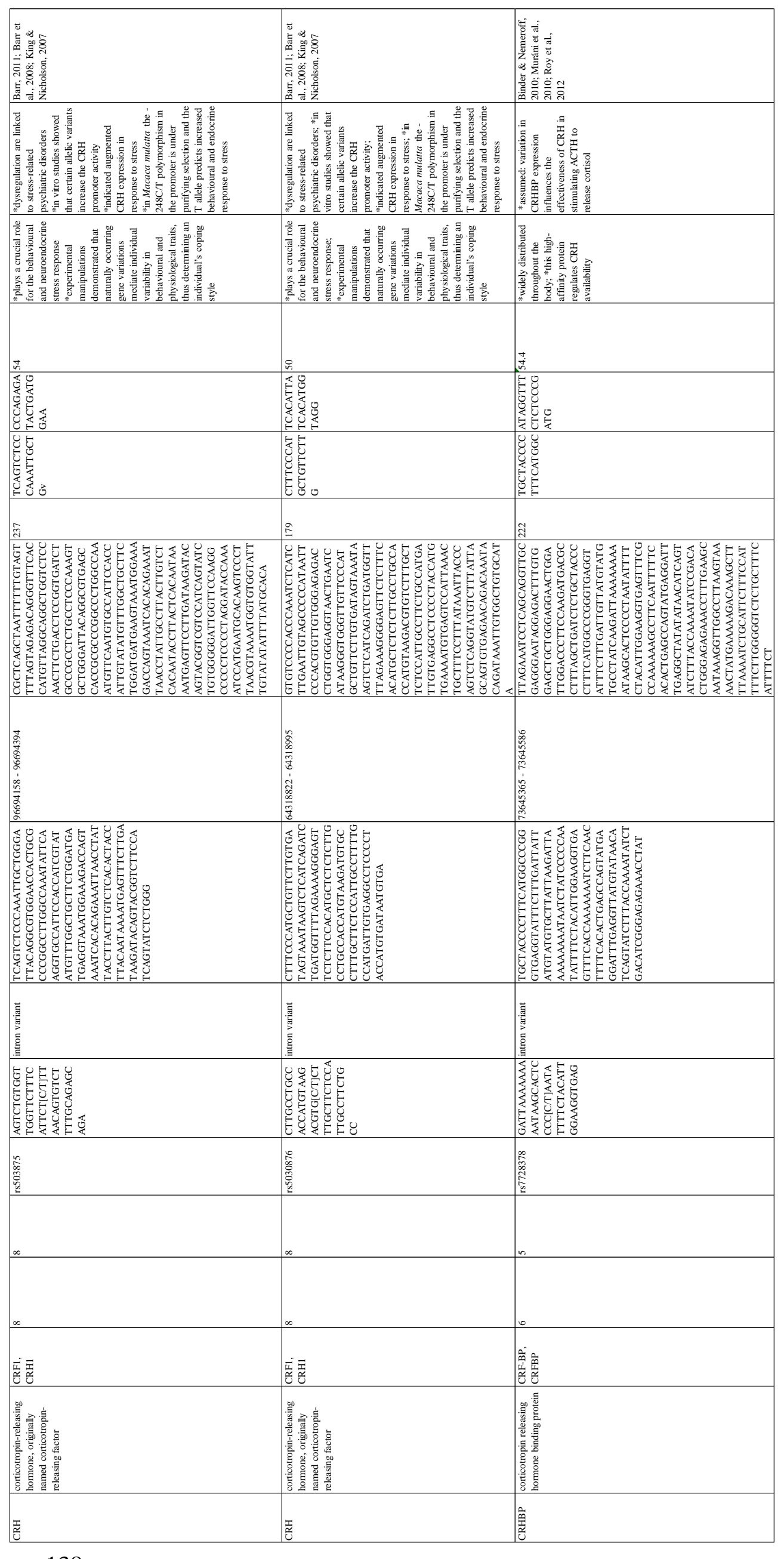

138 


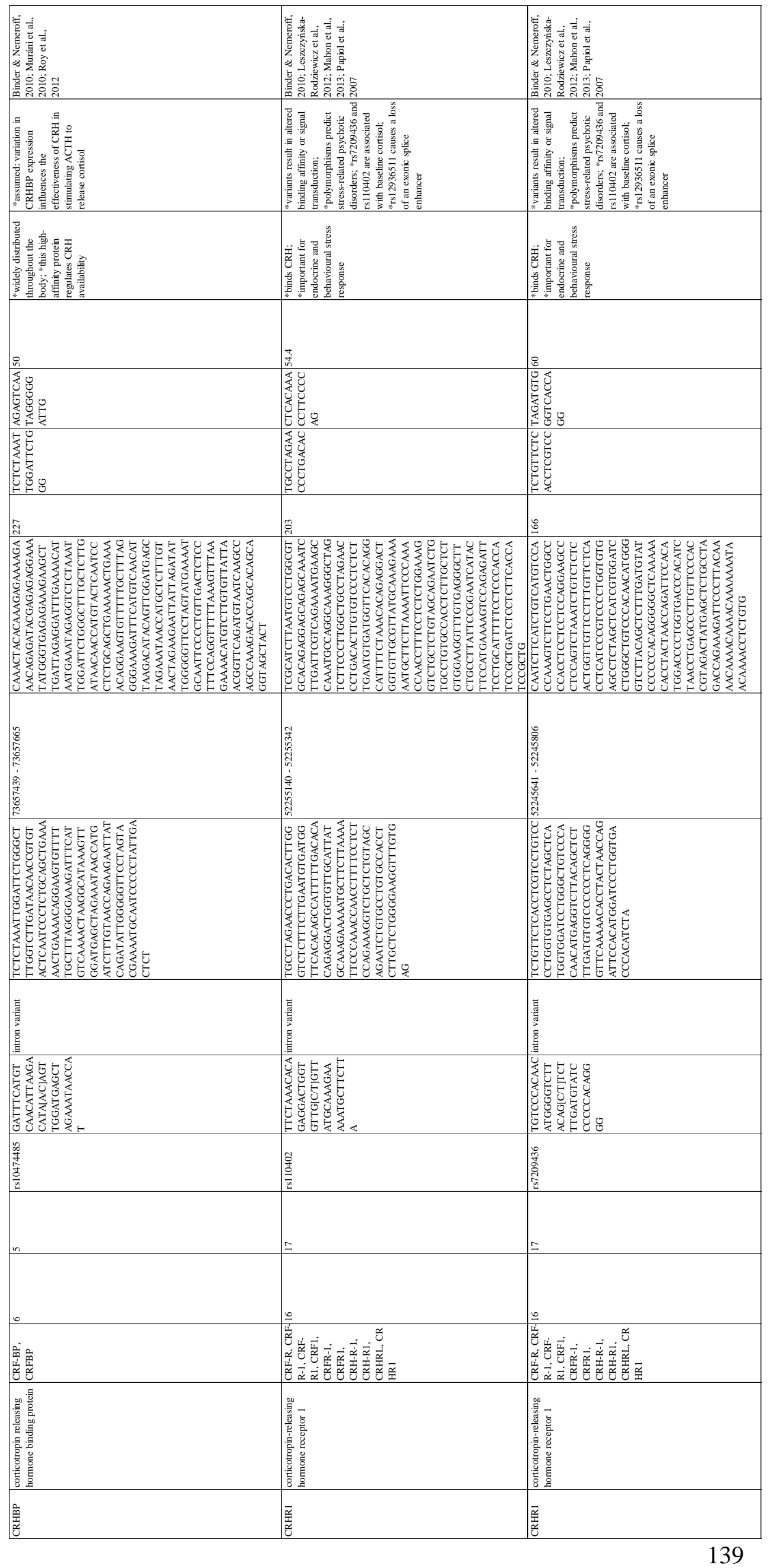




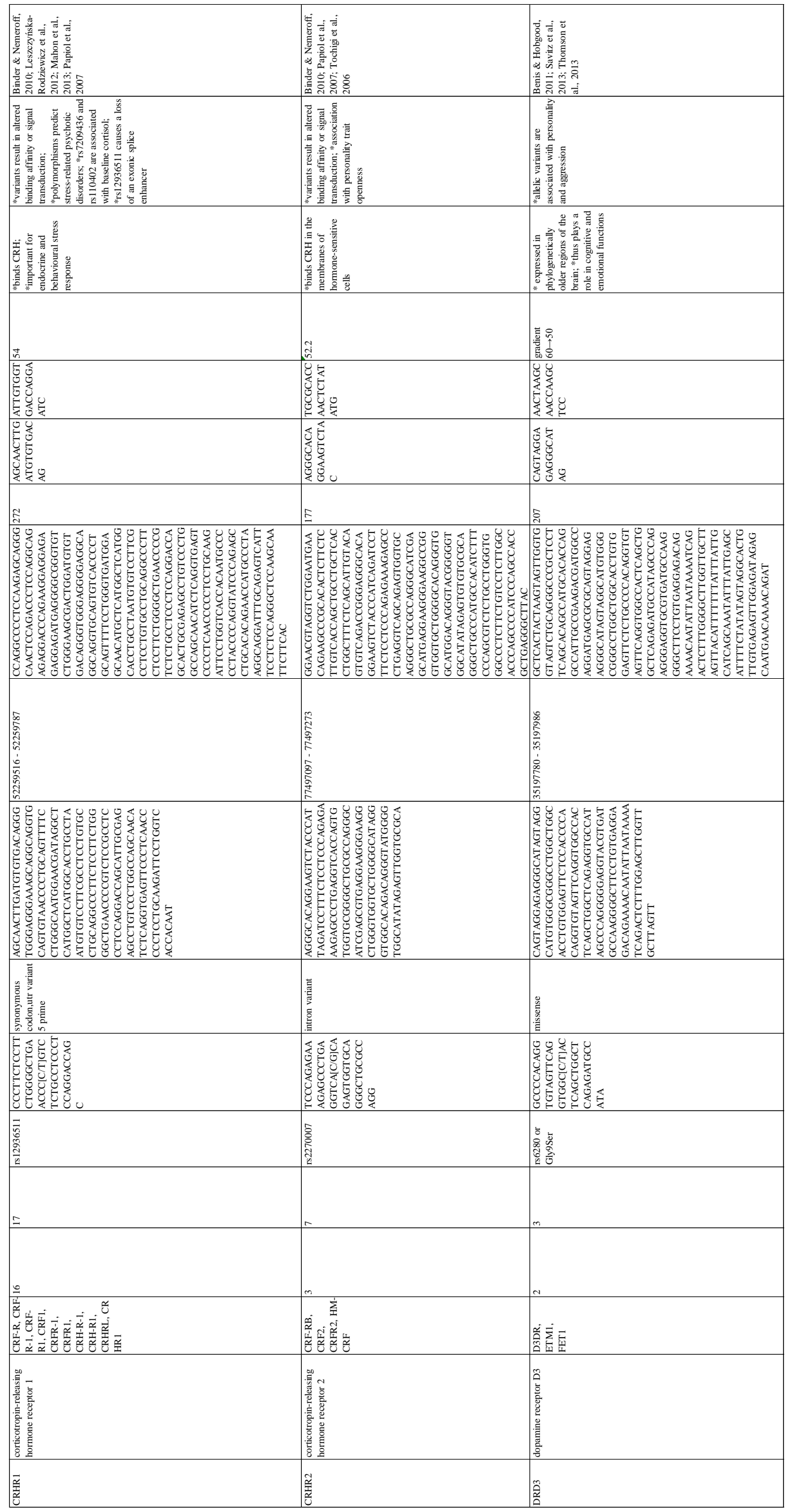




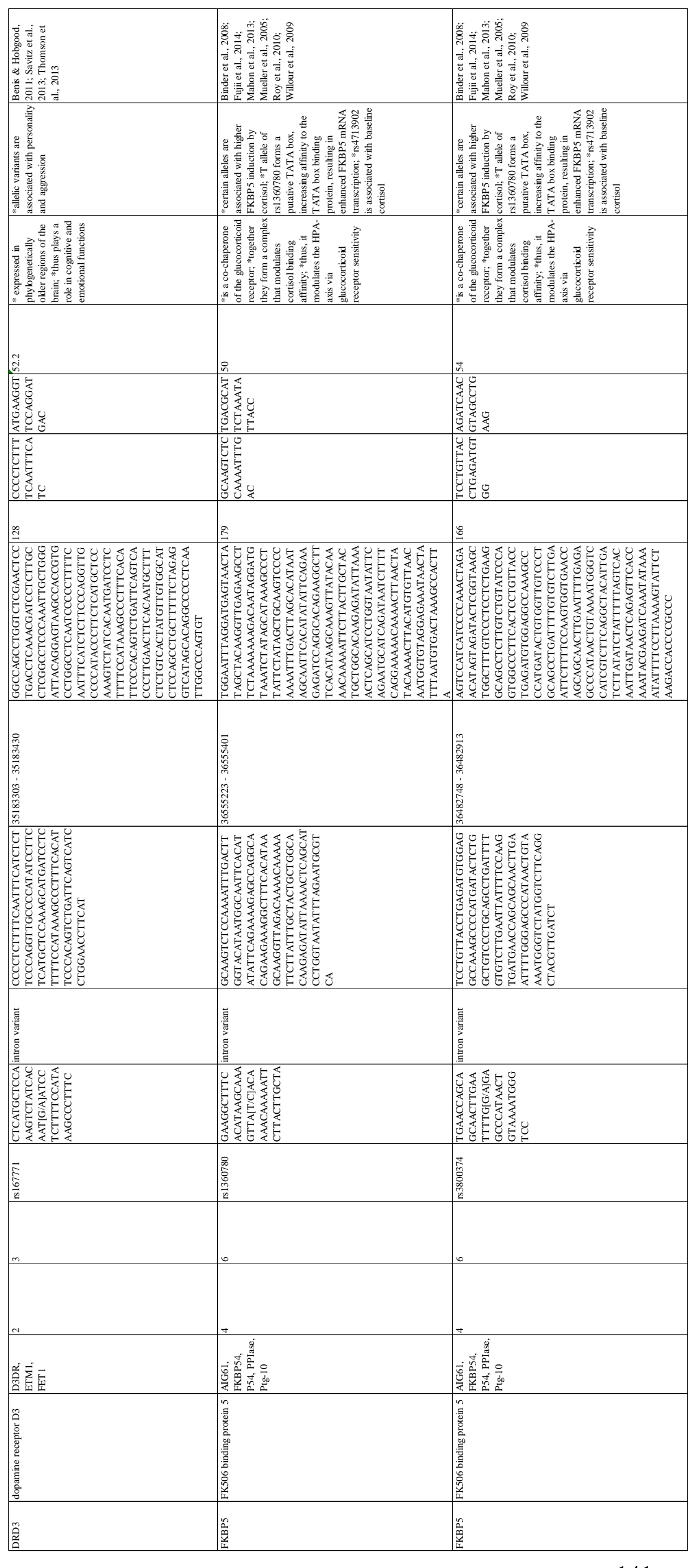




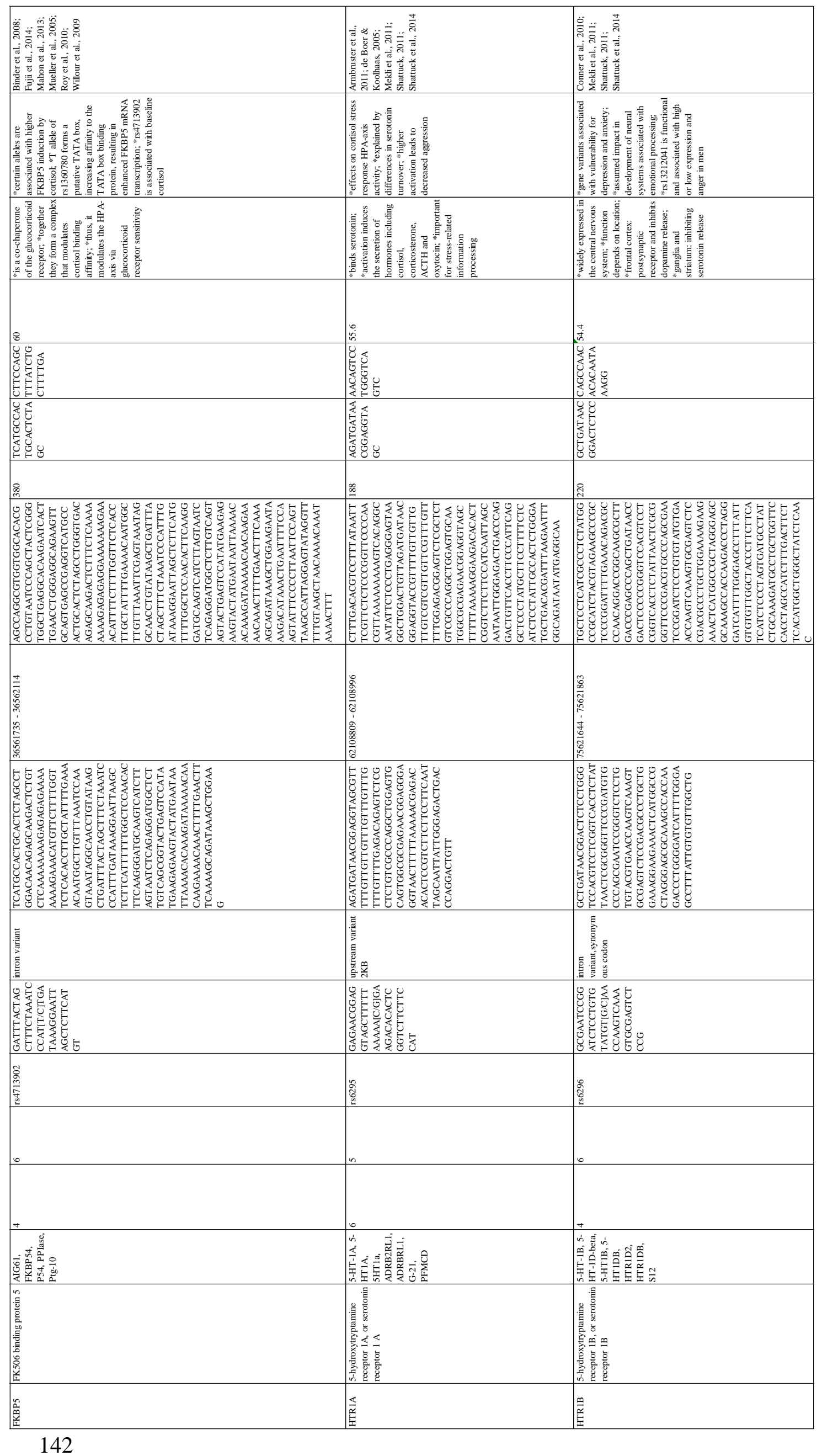




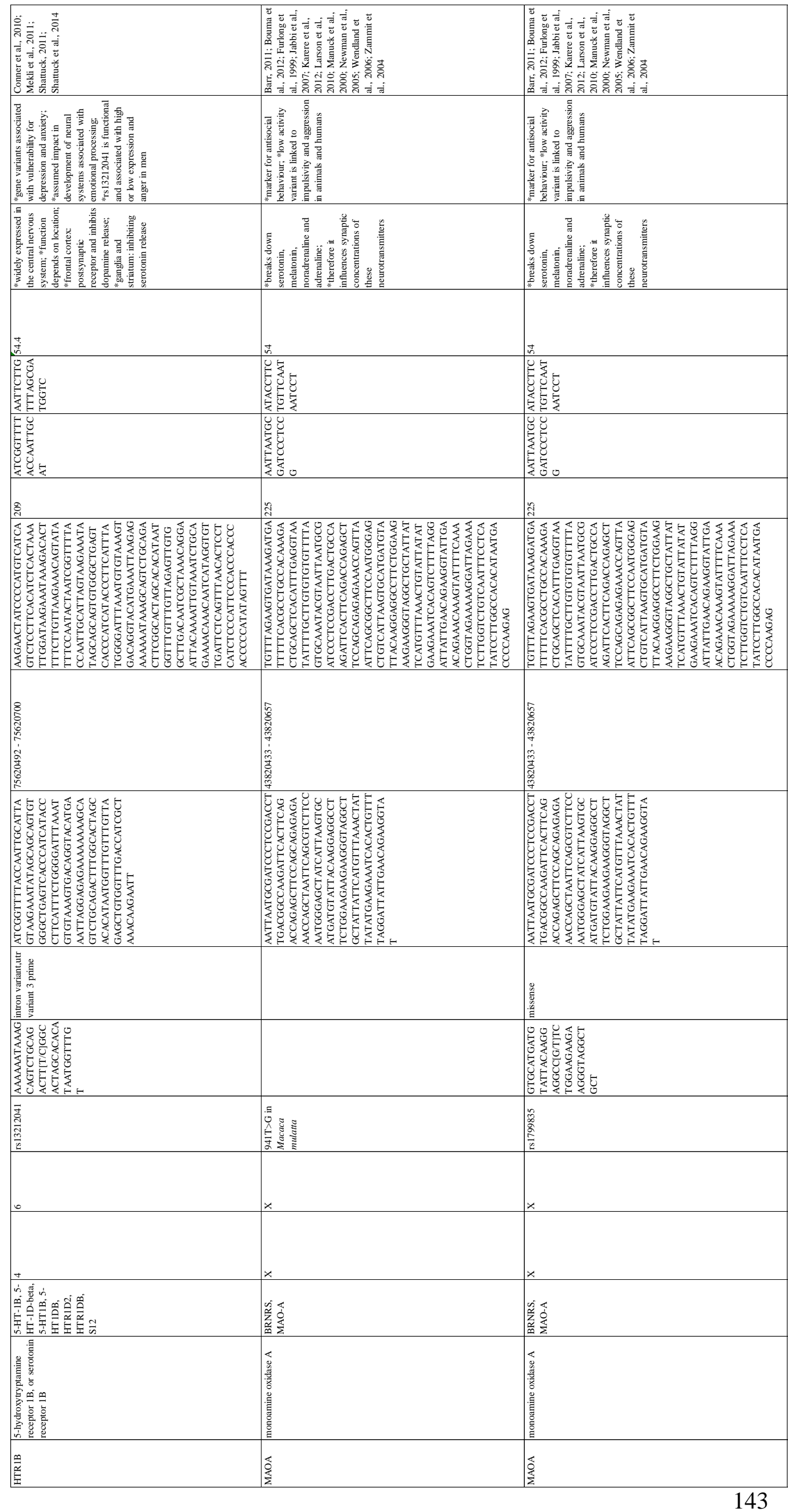




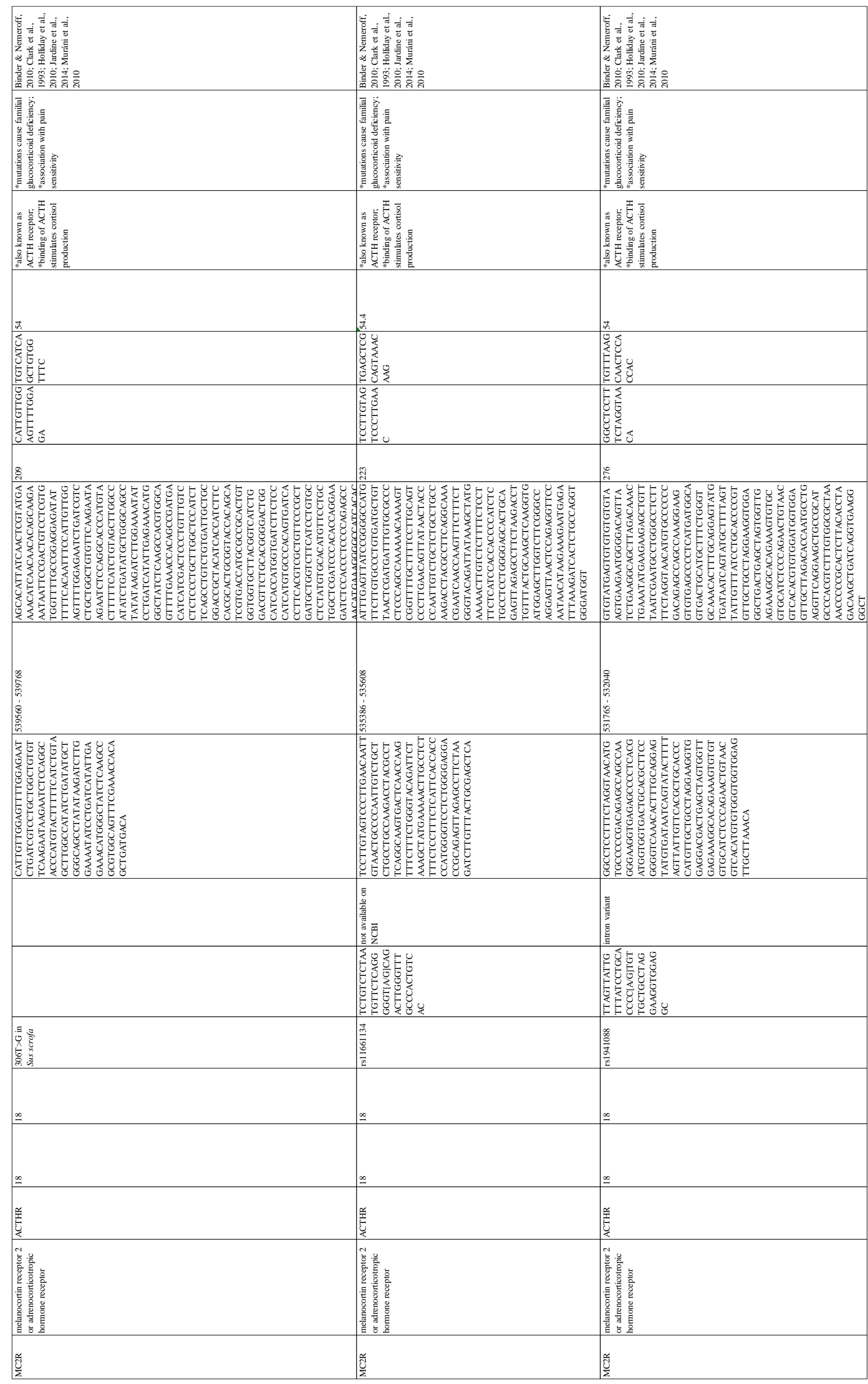




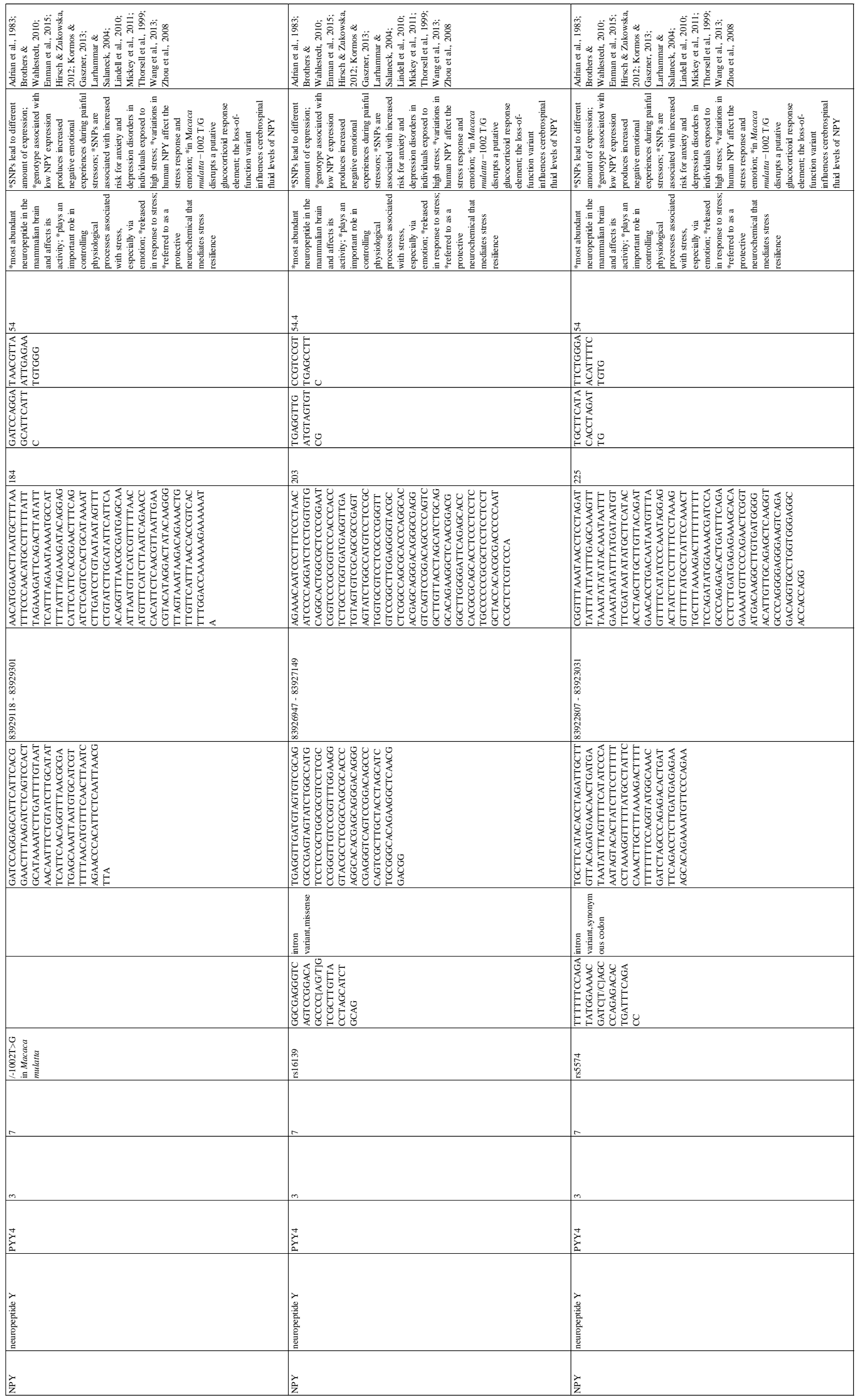




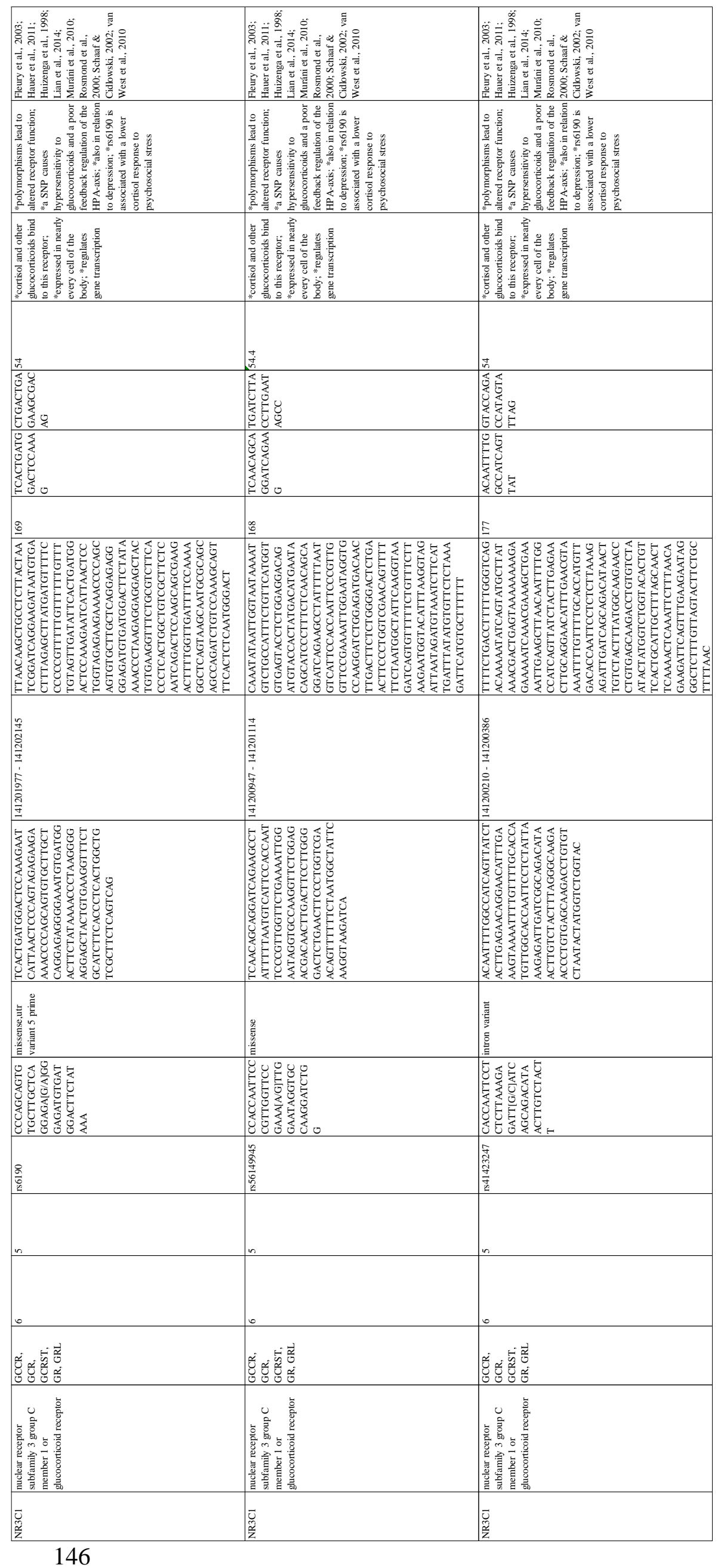




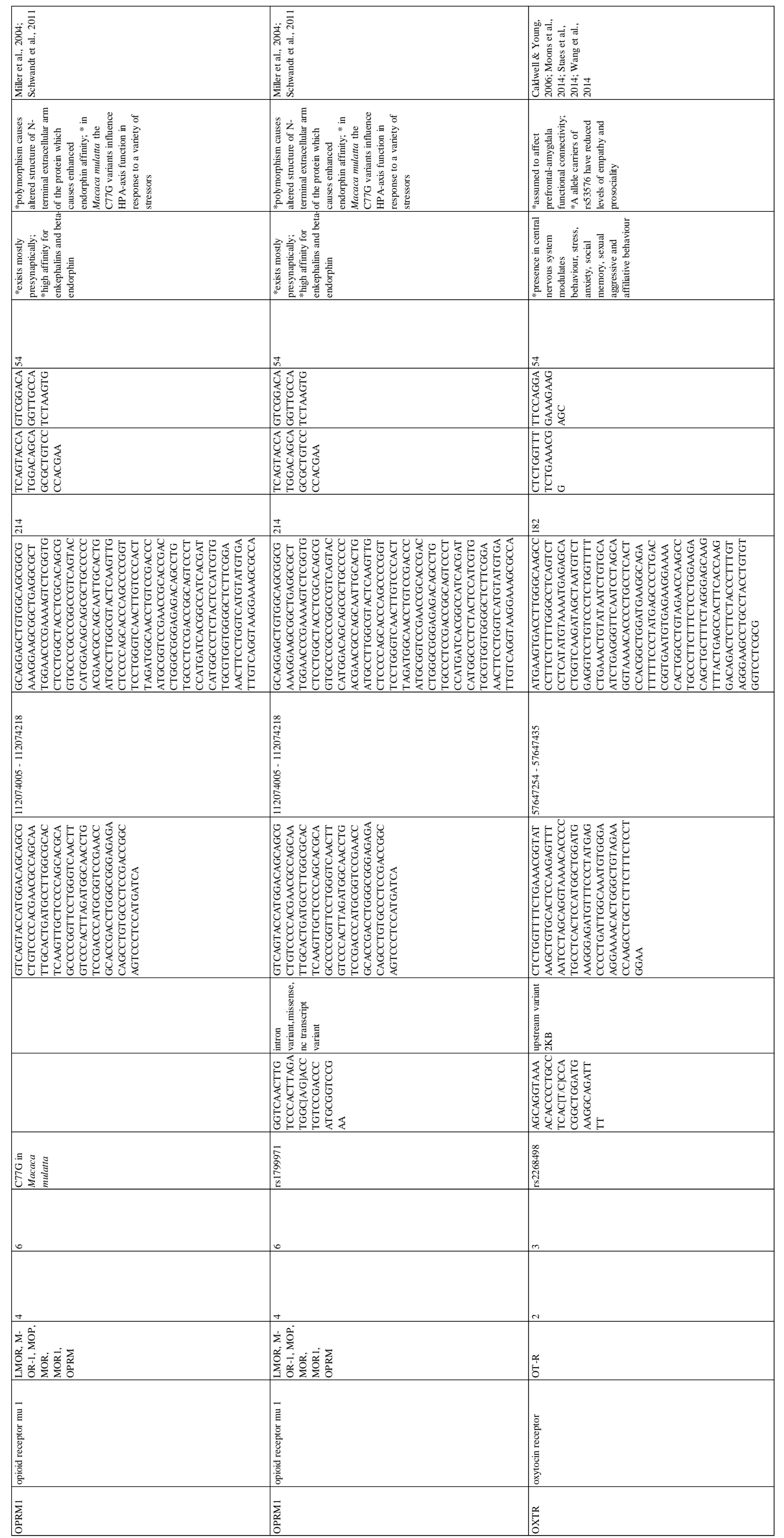




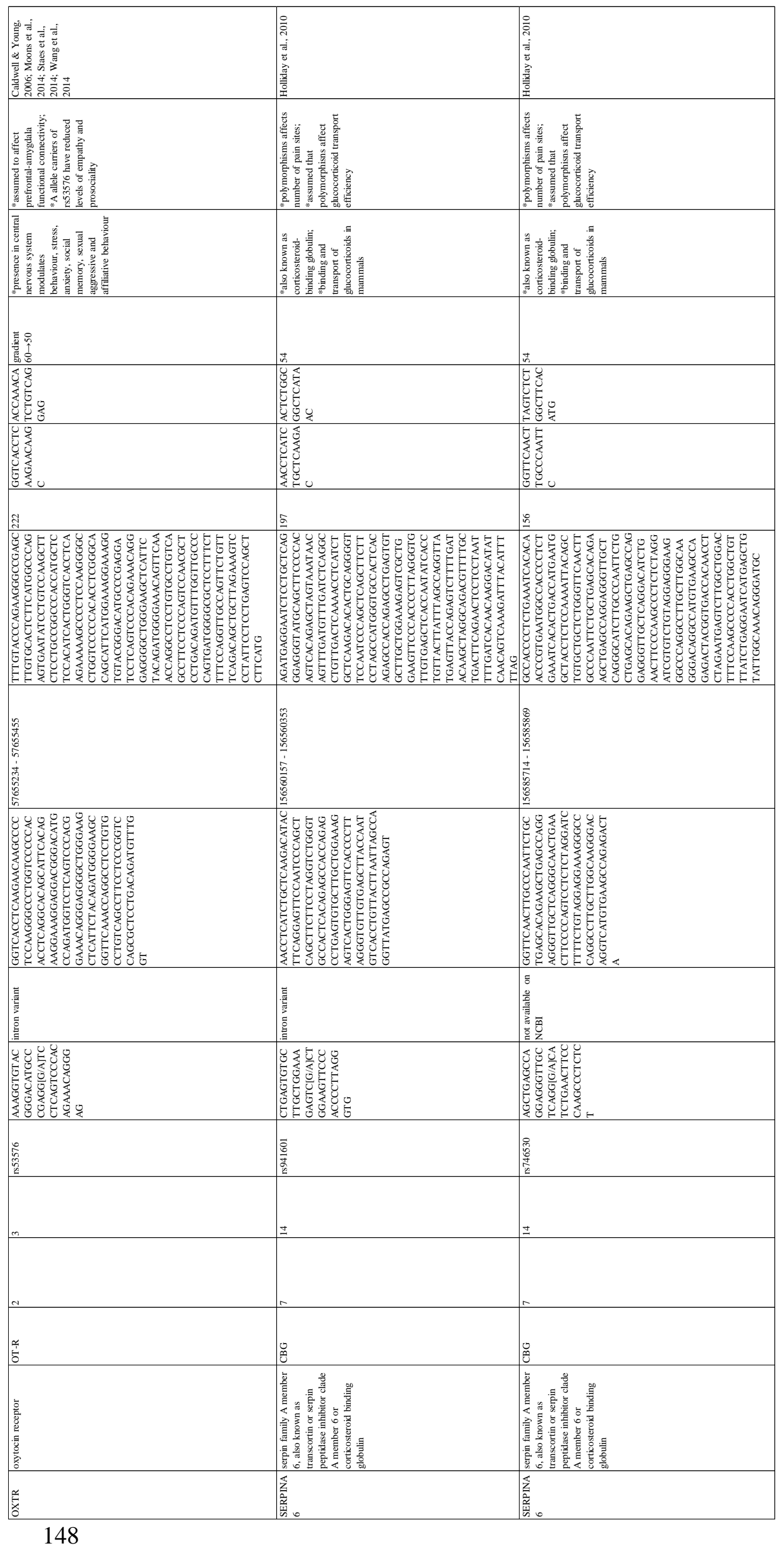




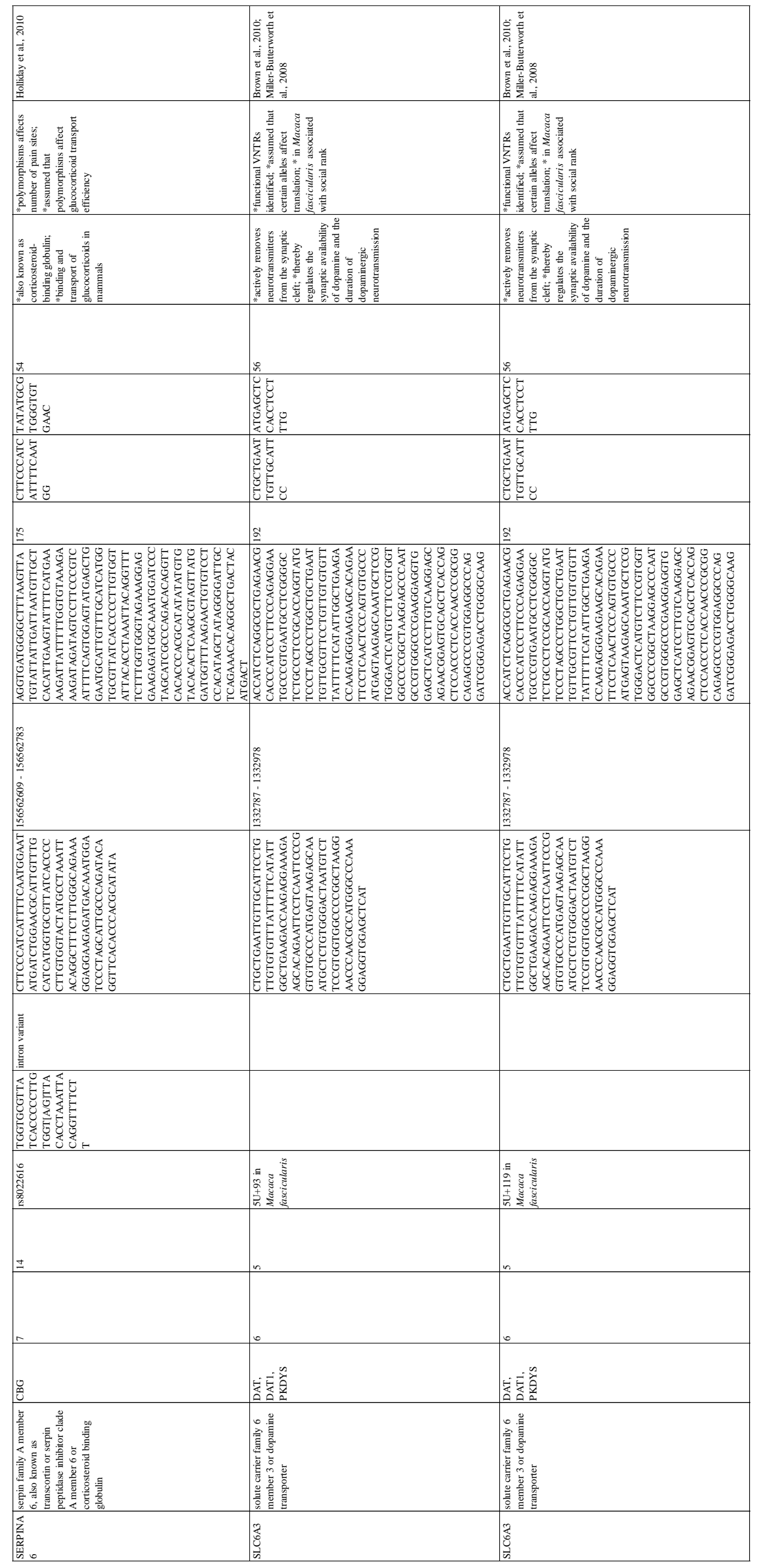




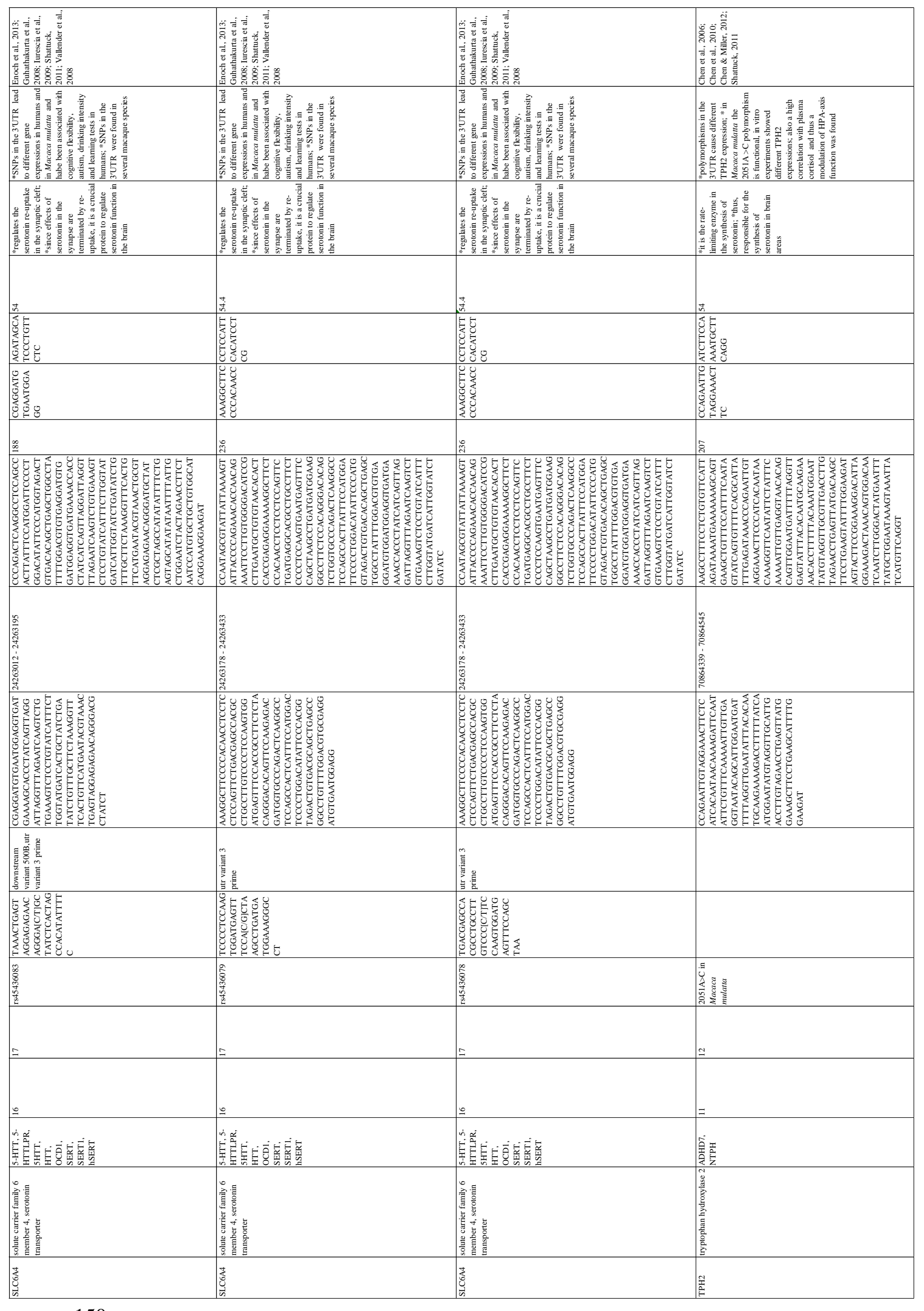


Table S1.2 Statistics of the next-generation sequencing

\begin{tabular}{|c|c|c|c|c|c|c|c|c|c|c|c|c|c|c|c|c|}
\hline & \# Raw & reads & Averag & ge qualit & ty of raw 1 & reads & \# Quality tri & nmed reads & Average Q & ality of qua & lity trimmed & reads & & & & \\
\hline & forward & reverse & forward & Stdev & reverse & Stdev & forward & reverse & forward & Stdev & reverse & Stdev & & & & Staev \\
\hline 1.00 & 147970 & 147970 & 37.24 & 3.17 & 36.93 & 3.19 & 131325 & 131325 & 37.55 & 3.16 & 37.44 & 3.16 & 130467 & 88.17 & 3590.59 & 2624.13 \\
\hline 2.00 & 145994 & 145994 & 37.28 & 3.18 & 36.05 & 3.11 & 136746 & 136746 & 37.65 & 3.17 & 36.93 & 3.13 & 136196 & 93.29 & 3560.17 & 2782.03 \\
\hline 3.00 & 135725 & 135725 & 37.32 & 3.19 & 36.70 & 3.17 & 122236 & 122236 & 37.74 & 3.17 & 37.35 & 3.15 & 122053 & 9.93 & 3017.96 & 2583.07 \\
\hline 4.00 & 148135 & 148135 & 37.39 & 3.18 & 36.69 & 3.17 & 116775 & 116775 & 37.81 & 3.17 & 37.36 & 3.14 & 116706 & 78.78 & 2935.78 & 2563.26 \\
\hline 5.00 & 159115 & 159115 & 37.24 & 3.17 & 36.97 & 3.17 & 145722 & 145722 & 37.54 & 3.16 & 37.46 & 3.17 & 145054 & 91.16 & 3961.50 & 2738.63 \\
\hline 6.00 & 179069 & 179069 & 37.28 & 3.20 & 36.64 & 3.19 & 169642 & 169642 & 37.63 & 3.18 & 37.18 & 3.16 & 166888 & 93.20 & 3663.64 & 2837.66 \\
\hline 7.00 & 128607 & 128607 & 37.31 & 3.22 & 36.87 & 3.18 & 107646 & 107646 & 37.68 & 3.22 & 37.40 & 3.16 & 96158 & 74.77 & 2650.97 & 2604.67 \\
\hline 8.00 & 179757 & 179757 & 37.49 & 3.19 & 35.94 & 3.07 & 149507 & 149507 & 37.89 & 3.18 & 36.65 & 3.10 & 149351 & 3.08 & 500.47 & 2676.28 \\
\hline 9.00 & 197181 & 197181 & 37.46 & 3.18 & 35.91 & 3.13 & 161805 & 161805 & 37.90 & 3.18 & 36.77 & 3.12 & 161023 & 1.66 & 585.77 & 2871.33 \\
\hline 10.00 & 132420 & 132420 & 37.43 & 3.19 & 36.87 & 3.15 & 111624 & 111624 & 37.79 & 3.19 & 37.43 & 3.14 & 111473 & 84.18 & 3168.96 & 2550.15 \\
\hline 11.00 & 153373 & 153373 & 37.45 & 3.24 & 37.04 & 3.16 & 125638 & 125638 & 37.79 & 3.23 & 37.53 & 3.16 & 124509 & 81.18 & 3424.33 & 2501.10 \\
\hline 12.00 & 161244 & 161244 & 37.50 & 3.18 & 36.97 & 3.16 & 153808 & 153808 & 37.83 & 3.18 & 37.41 & 3.15 & 153795 & 95.38 & 3274.24 & 2517.92 \\
\hline 13.00 & 126499 & 126499 & 37.34 & 3.18 & 35.56 & 3.07 & 106951 & 106951 & 37.82 & 3.17 & 36.55 & 3.10 & 106793 & 84.42 & 2471.86 & 2365.31 \\
\hline 14.00 & 108165 & 108165 & 37.40 & 3.20 & 36.83 & 3.25 & 91012 & 91012 & & & 37.38 & 20 & 90965 & 84.10 & 584.88 & 237 \\
\hline 15.00 & 196348 & 196348 & 37.60 & 3.22 & 37.34 & 3.20 & 186478 & 1864 & & & & & 186 & & .31 & 269 \\
\hline 16.00 & 203164 & 203164 & 37.50 & 3.20 & 36.67 & 3.15 & 14119 & 44119 & & & 37.29 & 15 & 17 & & 2954.15 & 2762 \\
\hline 17.00 & 170053 & 170053 & 37.50 & 3.20 & 36.66 & 3.18 & 22226 & 122226 & .85 & 18 & 37.30 & 15 & 122021 & 1.75 & 3161.34 & 2786 \\
\hline 18.00 & 133547 & 133547 & 37.43 & 3.19 & 36.79 & 3.18 & 113472 & 113472 & 7.87 & 3.19 & 37.44 & 3. & 113269 & 4.82 & 185.02 & 2485.95 \\
\hline 19.00 & 163172 & 163172 & 37.47 & 3.22 & 36.57 & 3.13 & 47963 & 147963 & 7.87 & 3.19 & 37.13 & 3.13 & 147936 & 0.66 & 573.16 & 2622.12 \\
\hline 20.00 & 132784 & 132784 & 37.54 & 3.20 & 36.93 & 3.25 & 125185 & 125185 & 38 & 3. & 37.41 & 3.2 & 1247 & 3.94 & 819.85 & 2694.12 \\
\hline 21.00 & 136210 & 136210 & 37.26 & 3.21 & 36.75 & 3.16 & 88489 & 88489 & 7.79 & 3.17 & 37.45 & 3.16 & 88472 & 4.95 & 129.47 & 2443.10 \\
\hline 22.00 & 145910 & 145910 & 37.48 & 3.18 & 36.40 & 3.12 & 136132 & 136132 & 37.84 & 3.19 & 37.02 & 3.13 & 135149 & 92.62 & 397.82 & 2741.71 \\
\hline 23.00 & 113759 & 113759 & 37.47 & 3.19 & 36.75 & 3.21 & 05388 & 105388 & 37.83 & 3.18 & 37.27 & 3.20 & 105 & 2.59 & 031.15 & 2491.93 \\
\hline 24.00 & 169325 & 169325 & 37.46 & 3.21 & 36.88 & 3.19 & 149656 & 149656 & 37.80 & 3.19 & 37.39 & 3.16 & 148745 & 87.85 & 3614.41 & 2832.48 \\
\hline 25.00 & 138301 & 138301 & 37.37 & 3.23 & 36.34 & 3.11 & 115228 & 115228 & 37.76 & 3.23 & 37.06 & 3.12 & 114143 & 82.53 & 3161.48 & 2543.31 \\
\hline 26.00 & 118662 & 118662 & 37.39 & 3.19 & 36.74 & 3.23 & 105811 & 105811 & & & & & 42 & & .90 & 245 \\
\hline 27.00 & 129041 & 129041 & 37.45 & 3.20 & 36.89 & 3.17 & 108883 & 1088 & & & & & 108 & & 93 & 251 \\
\hline 28.00 & 147664 & 147664 & 37.23 & 3.17 & 36.55 & 3.12 & 121259 & 1212 & & & 12 & & 120 & & 06 & 252 \\
\hline 29.00 & 126427 & 126427 & 37.38 & 3.19 & 36.75 & 3.17 & 00412 & 2 & 7.78 & 17 & 37.38 & & & 5.86 & .23 & 2429.36 \\
\hline 30.00 & 17656 & 176566 & 37.39 & 3.23 & 37.17 & 3.19 & 63713 & 3 & & 23 & 37.61 & 17 & 94 & 2.60 & 364.44 & 254 \\
\hline 31.00 & 135656 & 135656 & 37.33 & 3.23 & 35.81 & 3.08 & 11048 & 11048 & .80 & 3.24 & 36.68 & 3.10 & 110843 & 1.71 & 3052.85 & 2619.88 \\
\hline 32.00 & 152543 & 152543 & 37.46 & 3.19 & 37.13 & 3.19 & 33713 & 33713 & 7.81 & 3.18 & 37.61 & 3.17 & 131395 & 6.14 & 521.01 & 2758.09 \\
\hline 33.00 & 164721 & 164721 & 37.43 & 3.19 & 37.10 & 3.19 & 152620 & 152620 & 37.75 & 3.17 & 37.54 & 3.17 & 152568 & 2.62 & 3643.89 & 2770.60 \\
\hline 34.00 & 142473 & 142473 & 37.40 & 3.20 & 36.24 & 3.11 & 124161 & 124161 & 37.87 & 3.18 & 36.93 & 3.12 & 123756 & 6.86 & 976.56 & 2641.82 \\
\hline 35.00 & 148933 & 148933 & 37.33 & 3.18 & 35.52 & 3.08 & 128144 & 128144 & 37.79 & 3.19 & 36.51 & 3.10 & 127555 & 5.65 & 575.55 & 2675.39 \\
\hline 36.00 & 137793 & 137793 & 37.46 & 3.19 & 36.99 & 3.19 & 122230 & 122230 & 7.81 & 3.19 & 37.50 & 3.17 & 122193 & 88.68 & 287.79 & 2460.95 \\
\hline 37.00 & 114613 & 114613 & 37.52 & 3.20 & 36.44 & 3.13 & 108375 & 108375 & 37.87 & 3.19 & 36.98 & 3.13 & 108248 & 94.45 & 831.11 & 2567.99 \\
\hline 38.00 & 178353 & 178353 & 37.52 & 3.21 & 36.92 & 3.16 & 158054 & 158054 & 37.84 & 3.18 & 37.43 & 3.16 & 157343 & 88.22 & 592.10 & 2638.15 \\
\hline 39.00 & 132153 & 132153 & 37.46 & 3.19 & 36.83 & 3.24 & 114489 & 114489 & 37.84 & 3.19 & 37.40 & 3.20 & 113647 & 86.00 & 109.55 & 2503.59 \\
\hline 40.00 & 123710 & 123710 & 37.49 & 3.19 & 36.90 & 3.18 & 114879 & 114879 & 7.82 & 3.19 & 37.44 & 16 & 114849 & 2.84 & 225.58 & 2530 \\
\hline 41.00 & 191872 & 191872 & 37.39 & 3.18 & 37.17 & 3.20 & 156347 & 1563 & & & & & & & & \\
\hline 42.00 & 148470 & 148470 & 37.51 & 3.20 & 36.78 & 3.16 & 34864 & 34864 & 7.83 & 3.18 & 37.31 & 3.16 & 131357 & 8.47 & 3133.12 & 2737.49 \\
\hline 43.00 & 121515 & 121515 & 37.45 & 3.18 & 36.89 & 3.20 & 113078 & 113078 & 37.79 & 3.17 & 37.39 & 3.17 & 113027 & 93.01 & 2943.66 & 2676.79 \\
\hline 44.00 & 159759 & 159759 & 37.39 & 3.23 & 36.48 & 3.12 & 137126 & 137126 & 37.79 & 3.23 & 37.13 & 3.13 & 136114 & 85.20 & 3423.84 & 2686.67 \\
\hline 45.00 & 120832 & 120832 & 37.41 & 3.20 & 35.70 & 3.06 & 103662 & 103662 & 37.82 & 3.17 & 36.68 & 3.09 & 101890 & 4.32 & 2779.96 & 2442.00 \\
\hline 46.00 & 127201 & 127201 & 37.43 & 3.20 & 36.47 & 3.14 & 107679 & 107679 & 37.86 & 3.18 & 37.14 & 3.14 & 107648 & 4.63 & 2717.95 & 2590.50 \\
\hline 47.00 & 157432 & 157432 & 37.49 & 3.21 & 36.83 & 3.19 & 134841 & 134841 & 37.87 & 3.18 & 37.38 & 3.16 & 134141 & 5.21 & 409.95 & 2602.16 \\
\hline 48.00 & 165895 & 165895 & 37.44 & 3.20 & 37.11 & 3.20 & 152035 & 152035 & 37.80 & 3.18 & 37.61 & 3.17 & 151779 & 91.49 & 3382.58 & 2595.83 \\
\hline 49.00 & 124221 & 124221 & 37.48 & 3.20 & 36.75 & 3.23 & 118376 & 118376 & 37.82 & 3.17 & 37.23 & 3.20 & 118356 & 95.28 & 2860.26 & 2372.55 \\
\hline 50.00 & 155948 & 155948 & 37.51 & 3.20 & 37.01 & 3.18 & 135315 & 135315 & 37.84 & 3.18 & 37.52 & 3.16 & 122119 & 78.31 & 3443.55 & 2582.67 \\
\hline 51.00 & 91108 & 91108 & 37.31 & 3.18 & 35.88 & 3.12 & 82100 & 82100 & 37.70 & 3.16 & 36.82 & 3.12 & 82082 & 90.09 & 2171.53 & 1931.28 \\
\hline 52.00 & 162035 & 162035 & 37.58 & 3.21 & 36.73 & 3.13 & 145706 & 145706 & 7.92 & 3.19 & 7.22 & 3.13 & 144926 & 9.44 & 117.24 & 2490.46 \\
\hline 53.00 & 114559 & 114559 & 37.51 & 3.19 & 36.92 & 3.16 & 106965 & 106965 & 37.84 & 3.19 & 37.44 & 3.15 & 106905 & 3.32 & 197.46 & 2363.38 \\
\hline 54.00 & 253791 & 253791 & 37.46 & 3.22 & 37.11 & 3.21 & 12865 & 12865 & .87 & 18 & 37.65 & 17 & & .74 & 10.03 & 2886.00 \\
\hline 55.00 & 168003 & 168003 & 37.41 & 3.19 & 36.58 & 3.19 & 13034 & 13034 & .79 & 3.18 & 37.17 & 3.19 & & 4.97 & 653.58 & 260 \\
\hline 56.00 & 126210 & 126210 & 37.31 & 3.23 & 36.76 & 3.23 & 106926 & 06926 & & 3.23 & 37.34 & 19 & 106476 & 4.36 & 217.34 & 2469.21 \\
\hline 57.00 & 155544 & 155544 & 37.50 & 3.19 & 36.37 & 3.11 & 23701 & 23701 & 37.88 & 3.19 & 37.00 & 3.12 & 122487 & 8.75 & 3592.76 & 2394.38 \\
\hline 58.00 & 158158 & 158158 & 37.31 & 3.24 & 36.69 & 3.19 & 138165 & 138165 & 37.80 & 3.19 & 37.38 & 3.16 & 137237 & 86.77 & 3766.74 & 2675.88 \\
\hline 59.00 & 171344 & 171344 & 37.44 & 3.19 & 36.40 & 3.10 & 148190 & 48190 & 37.85 & 3.19 & 37.02 & 3.12 & 147059 & 85.83 & 432.30 & 2767.70 \\
\hline 60.00 & 109671 & 109671 & 37.29 & 3.23 & 36.21 & 3.15 & 01845 & 1845 & 7.69 & 3.22 & 36.86 & 3.17 & 101019 & 2.11 & 077.65 & 2543.13 \\
\hline 61.00 & 159067 & 159067 & 37.37 & 3.18 & 36.00 & 3.10 & 130665 & 130665 & 37.76 & 3.18 & 36.87 & 3.11 & 127816 & 80.35 & 205.91 & 2443.67 \\
\hline 62.00 & 148607 & 148607 & 37.44 & 3.17 & 36.79 & 3.14 & 128077 & 128077 & 37.80 & 3.17 & 37.32 & 3.15 & 126028 & 84.81 & 3055.91 & 2667.39 \\
\hline 63.00 & 163680 & 163680 & 37.43 & 3.18 & 36.62 & 3.16 & 149199 & 149199 & 37.82 & 3.17 & 37.24 & 3.15 & 149191 & 91.15 & 3541.75 & 2620.18 \\
\hline 64.00 & 182463 & 182463 & 37.46 & 3.20 & 36.84 & 3.17 & 152414 & 152414 & 37.83 & 3.19 & 37.45 & 3.15 & 151983 & 83.30 & 3763.62 & 2772.60 \\
\hline 65.00 & 150424 & 150424 & 37.42 & 3.24 & 36.88 & 3.16 & 115813 & 115813 & 37.77 & 3.24 & 37.43 & 3.16 & 115762 & 76.96 & 3040.86 & 2630.40 \\
\hline 66.00 & 140989 & 140989 & 37.45 & 3.19 & 35.85 & 3.08 & 115032 & & 37.90 & 3.19 & 36.71 & 3.11 & 114955 & 81.53 & 3029.87 & 2436.77 \\
\hline 67.00 & 175165 & 175165 & 37.35 & 3.23 & 36.82 & 3.18 & 141476 & 41476 & 7.74 & 3.23 & 37.45 & 3.15 & 141206 & 80.61 & 3479.75 & 2571.27 \\
\hline 68.00 & 126525 & 126525 & 37.47 & 3.18 & 36.09 & 3.11 & 109513 & 09513 & & & 36.88 & & & 85.05 & 2773.26 & 2394.78 \\
\hline 69.00 & 177643 & 177643 & 37.54 & 3.19 & 37.12 & 3.17 & 150943 & 50943 & & & 37.58 & 3.16 & & 84.75 & 217.36 & 2443.40 \\
\hline 70.00 & 165003 & 165003 & 37.41 & 3.19 & 36.10 & 3.10 & 131961 & 31961 & & & 36.98 & & 128966 & 78.16 & 291.10 & 2515.47 \\
\hline 71.00 & 127068 & 127068 & 37.43 & 3.20 & 36.88 & 3.23 & 116070 & 16070 & 7.80 & 3.18 & 37.42 & 3.20 & 116030 & 91.31 & 843.53 & 2449.32 \\
\hline 72.00 & 138847 & 138847 & 37.42 & 3.20 & 36.86 & 3.16 & 104331 & 31 & & 3.17 & 7.46 & 3.15 & 104155 & 75.01 & 2853.26 & 2622.76 \\
\hline 73.00 & 139112 & 139112 & 37.41 & 3.23 & 36.55 & 3.11 & 125434 & 125434 & 37.80 & 3.24 & 37.10 & 3.12 & 118443 & 85.14 & 3470.30 & 2709.25 \\
\hline 74.00 & 138744 & 138744 & 37.34 & 3.19 & 36.58 & 3.15 & 119574 & 119574 & 37.74 & 3.17 & 37.23 & 3.15 & 118115 & 85.13 & 3082.45 & 2562.54 \\
\hline 75.00 & 185374 & 185374 & 37.37 & 3.17 & 35.70 & 3.11 & 134998 & 134998 & 37.85 & 3.17 & 36.67 & 3.10 & 134852 & 72.75 & 3160.02 & 2479.13 \\
\hline 76.00 & 138696 & 138696 & 37.39 & 3.24 & 36.96 & 3.16 & 108229 & 108229 & 37.77 & 3.24 & 37.54 & 3.15 & 108183 & 78.00 & 3133.95 & 2636.24 \\
\hline 77.00 & 134229 & 134229 & 37.50 & 3.19 & 37.02 & 3.16 & 119384 & 119384 & 37.83 & 3.19 & 37.52 & 3.16 & 118894 & 88.58 & 3081.90 & 2651.62 \\
\hline 78.00 & 124752 & 124752 & 37.48 & 3.19 & 36.36 & 3.14 & 113533 & 113533 & 37.85 & 3.18 & 37.05 & 3.12 & 113187 & 90.73 & 3066.81 & 2505.02 \\
\hline 79.00 & 167374 & 167374 & 37.50 & 3.19 & 36.61 & 3.14 & 152720 & 152720 & 37.85 & 3.19 & 37.19 & 3.13 & 152409 & 91.06 & 3589.11 & 2858.33 \\
\hline & 11802465 & 11802465 & & & & & 10154750 & 10154750 & & & & & 10068490 & 85.31 & 3219.81 & \\
\hline
\end{tabular}




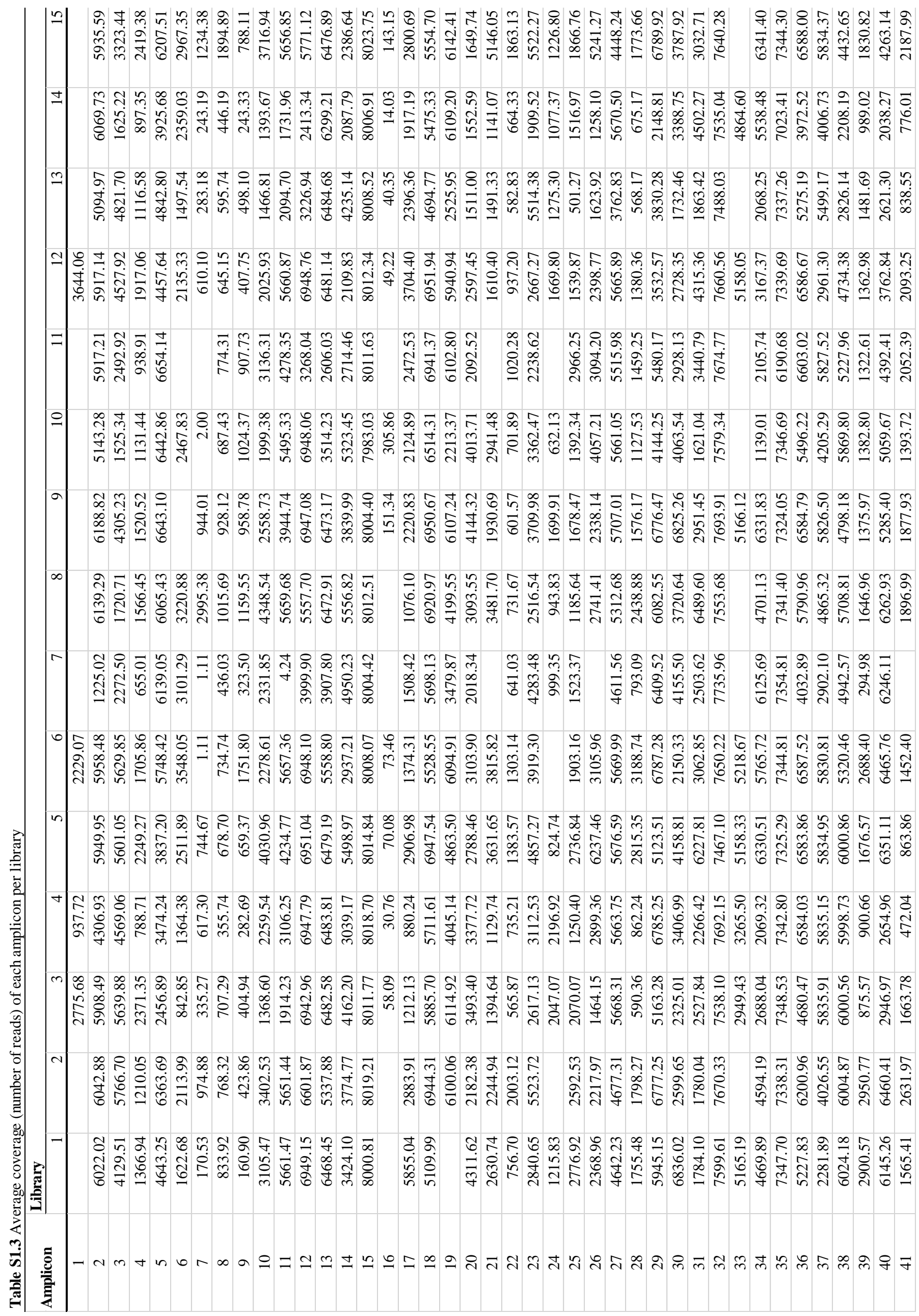




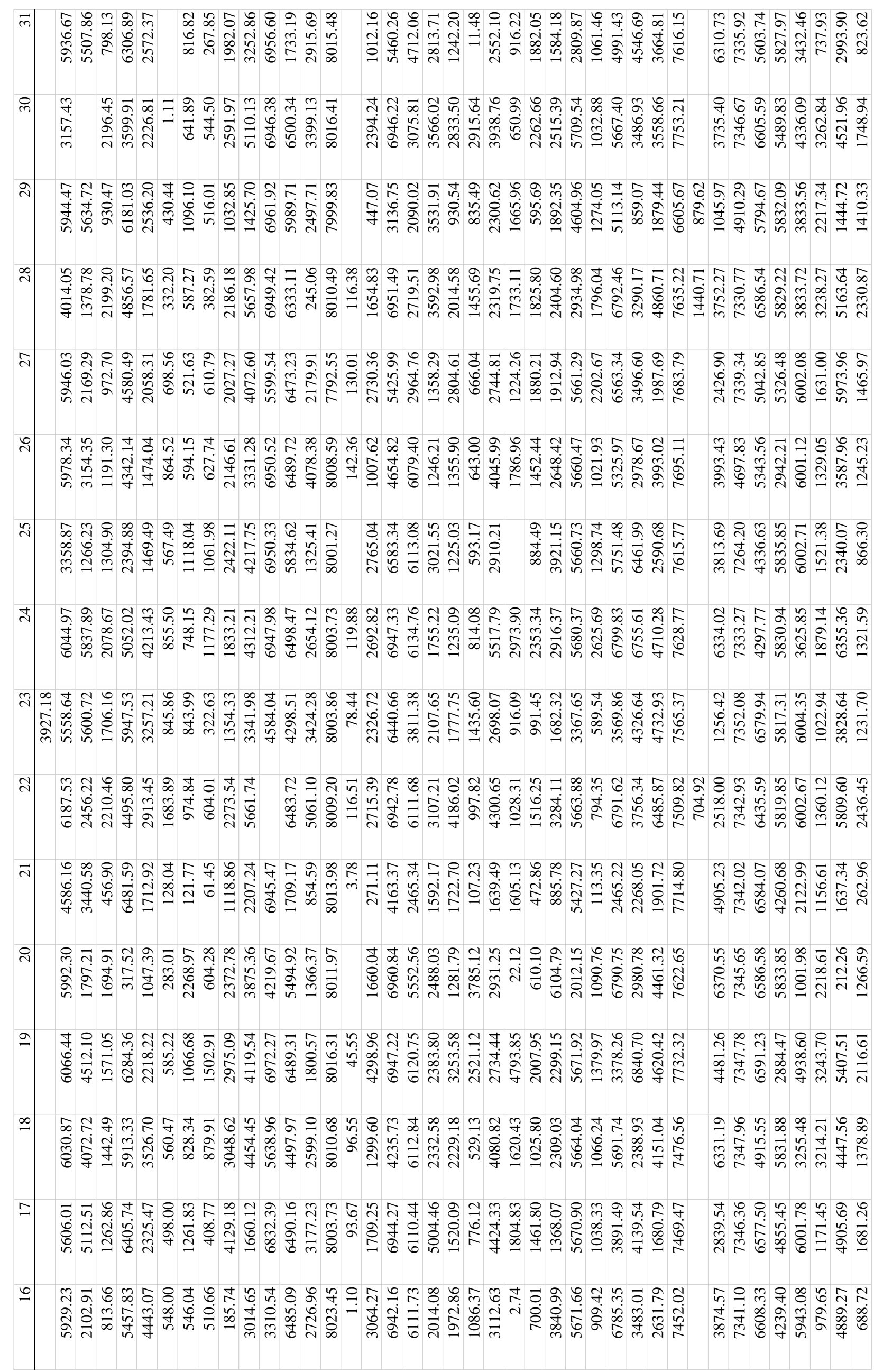




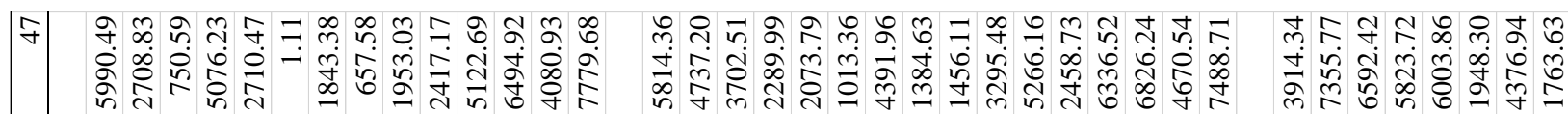

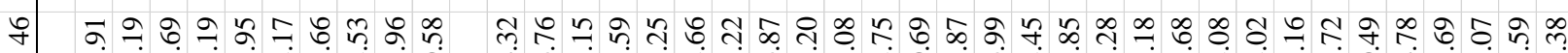

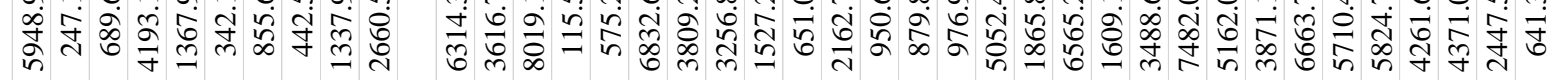

ケ처융

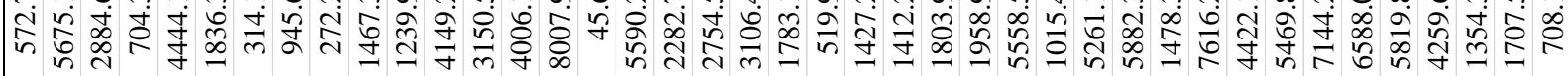

Ұ †क ๆ

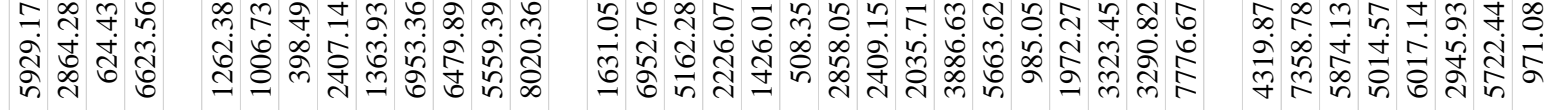

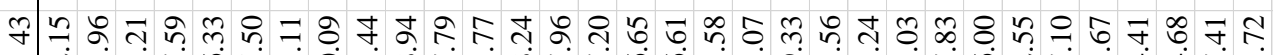

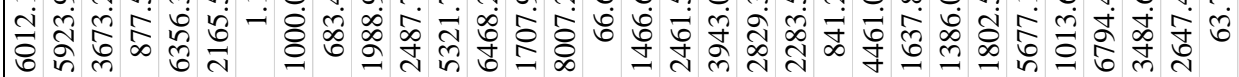

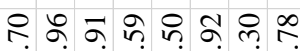

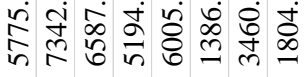

ᄀ 둥

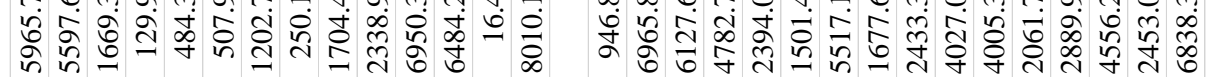

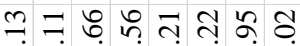

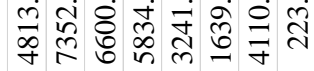

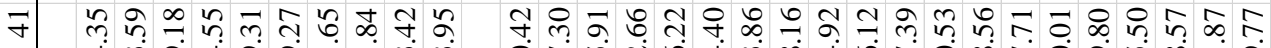

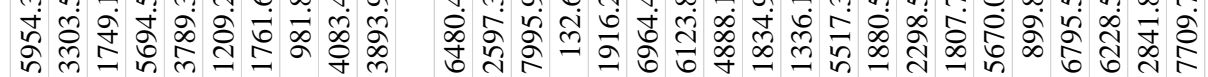

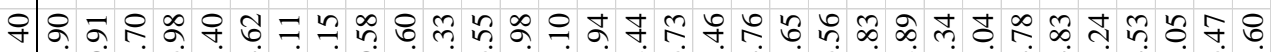

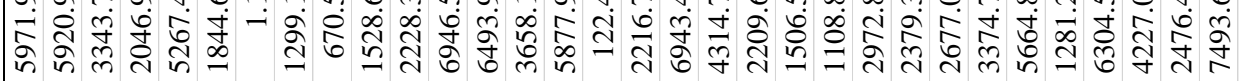

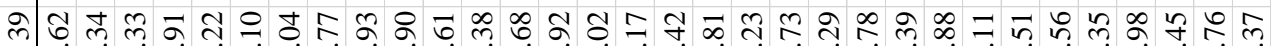

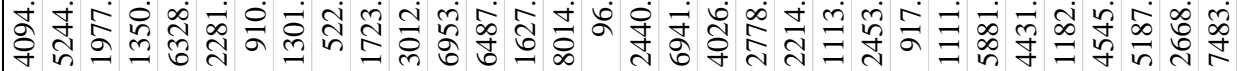

m

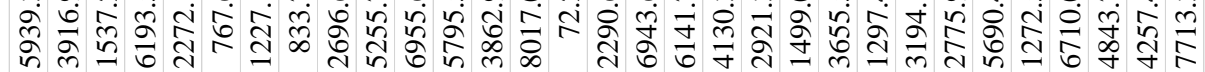

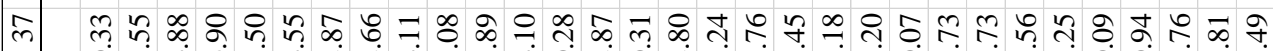

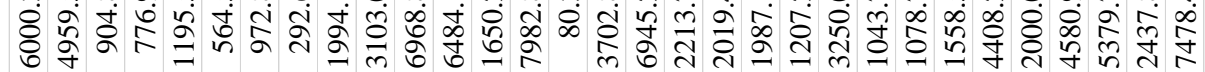

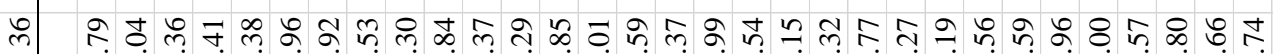

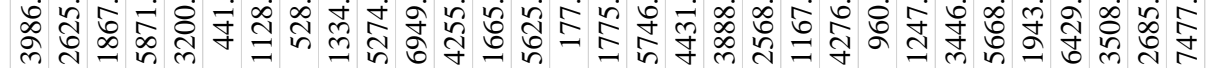

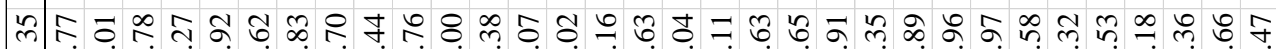

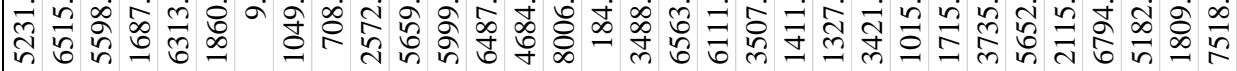

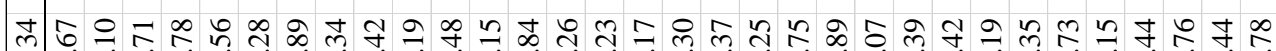

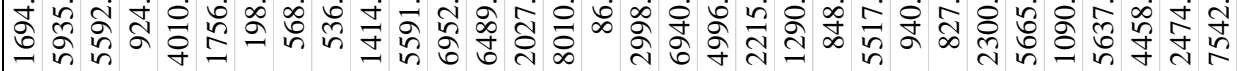

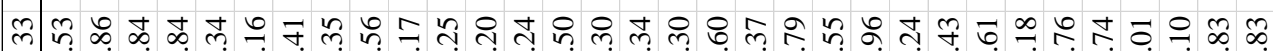

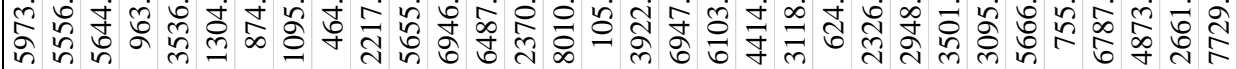

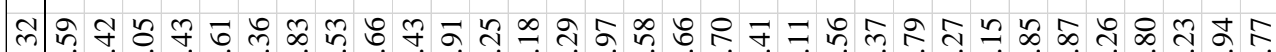

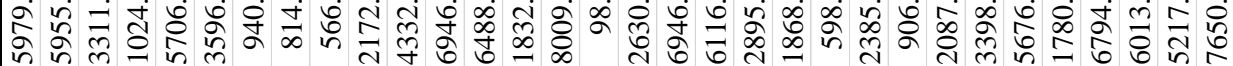

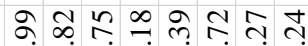
ถู่

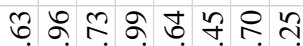

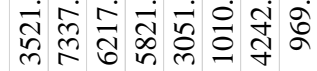

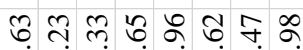
तुં

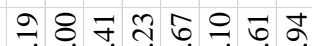

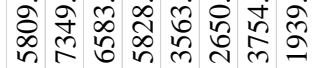

๑ ㅇำ

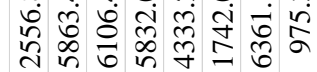

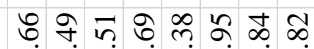
ڤై

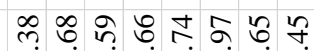

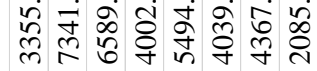

ำ ल

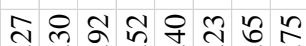
હું

ㄷำ

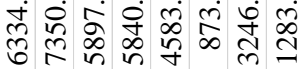




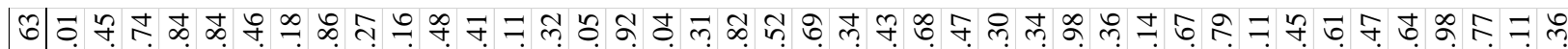

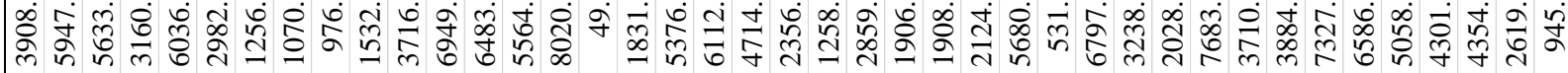

ช

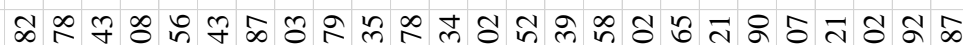

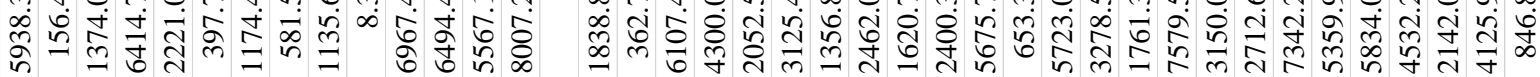

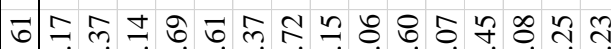

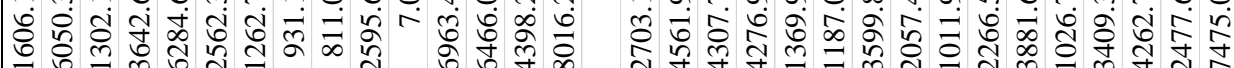

œ लెं

8 ๑

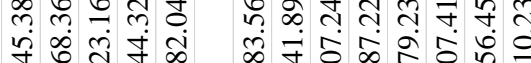

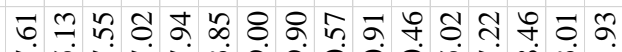

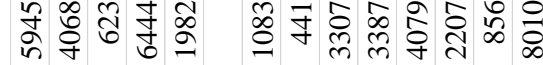

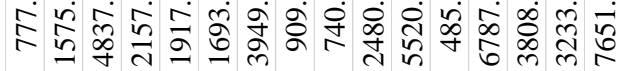

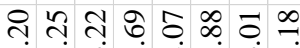

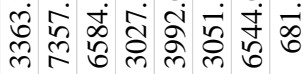

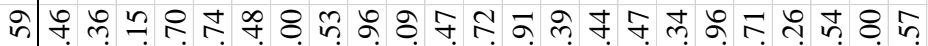

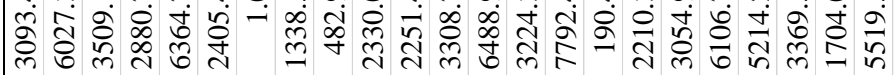
노요

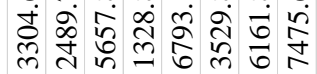

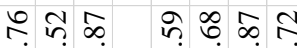

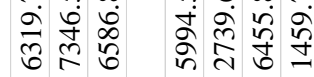

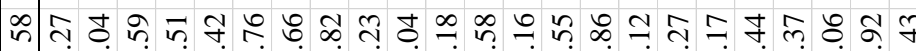

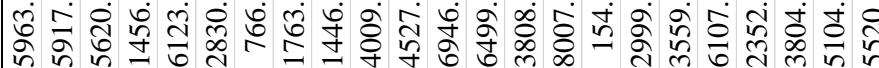

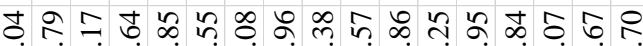

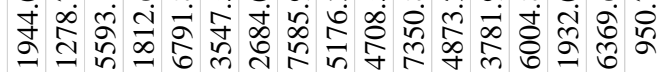

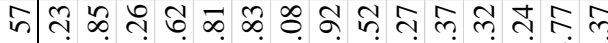

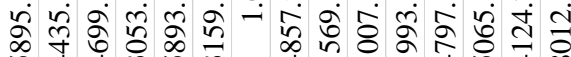

的称

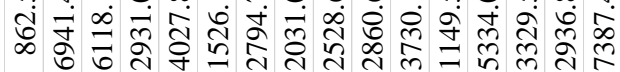

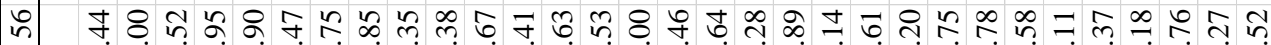

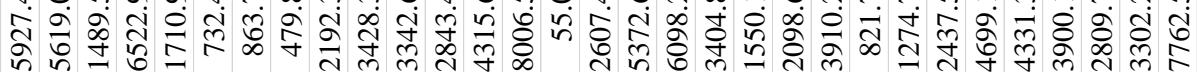

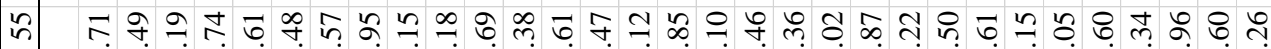

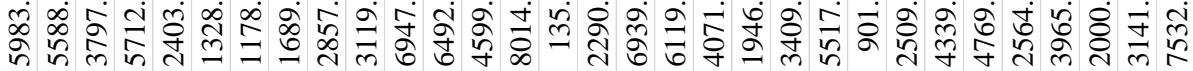

म 8 के

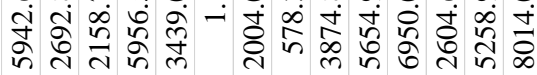

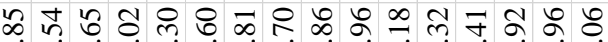

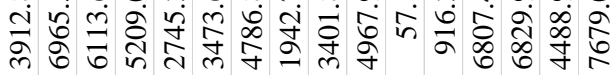

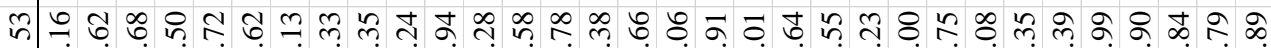

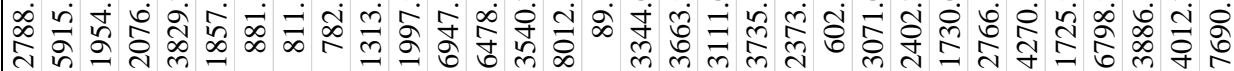

ก

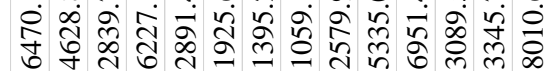

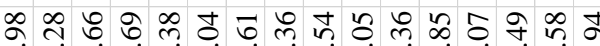

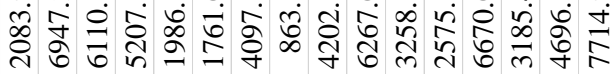

유워 웅 ำ

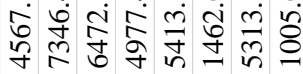

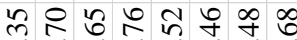

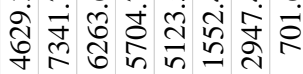

So in

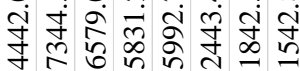

ชำㅇำ तิ હું

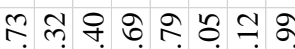

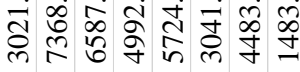

$\vec{\infty}$ 유 $\stackrel{\infty}{\infty}$ iิ

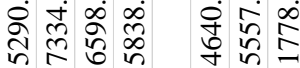

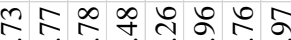

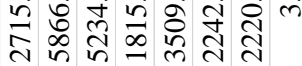

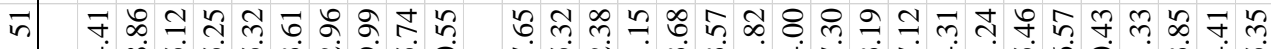

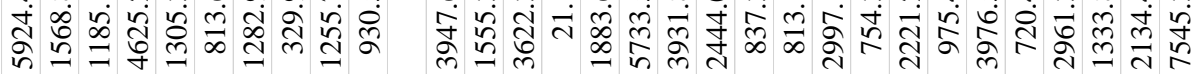

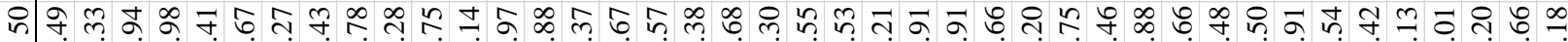

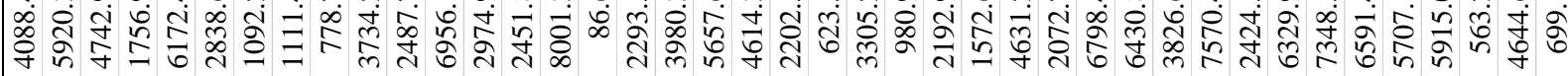

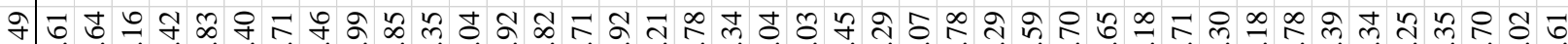

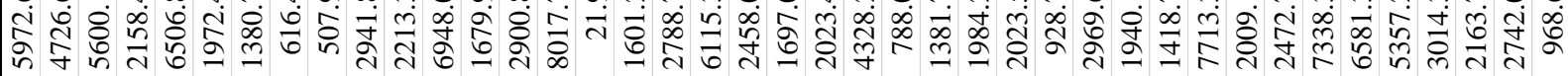

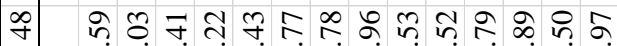

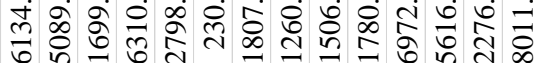

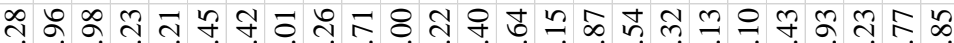

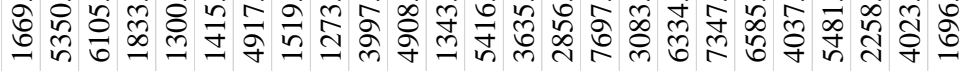




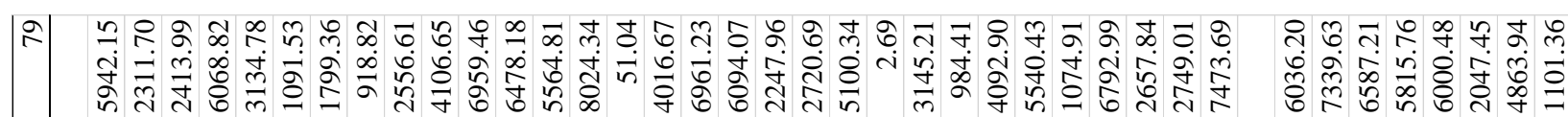

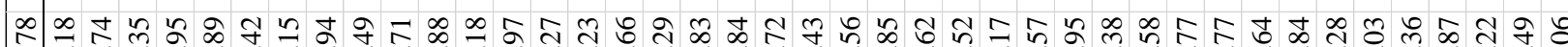

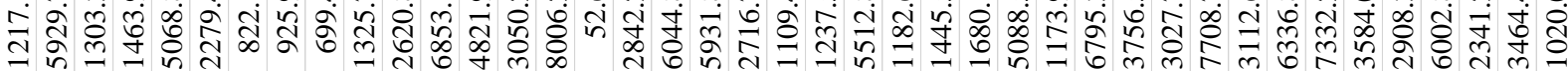

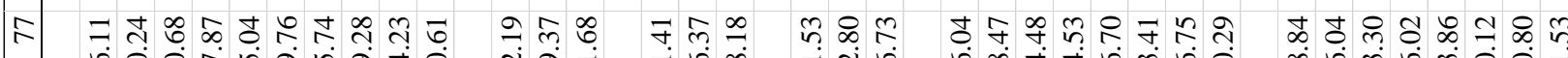

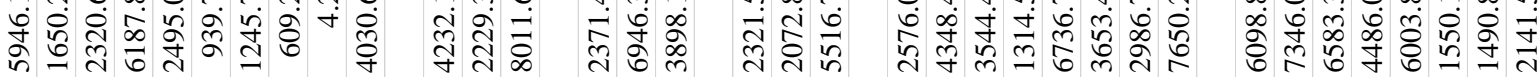

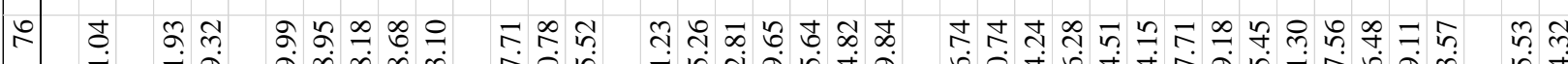

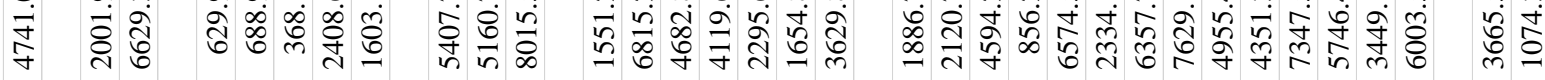

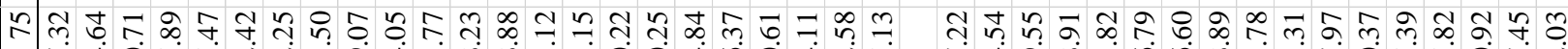

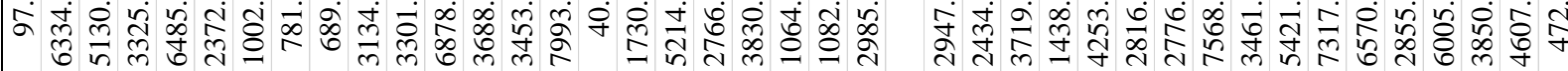

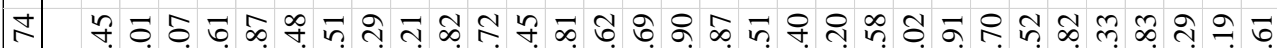

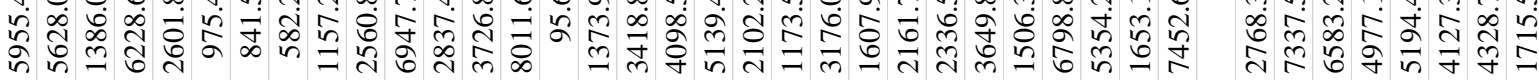

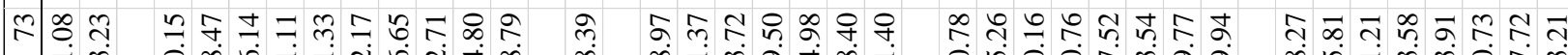

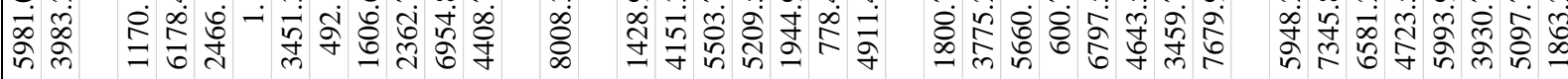

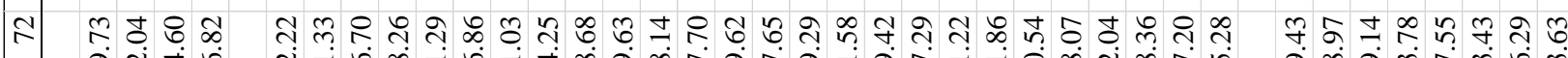

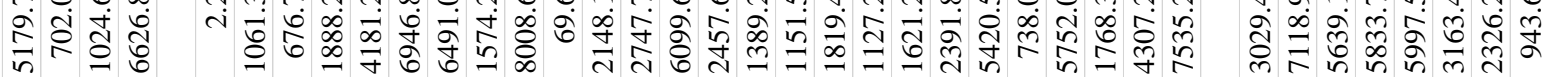

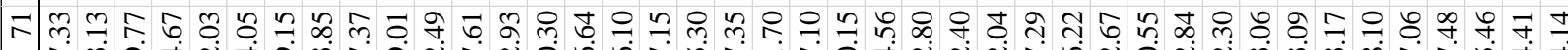

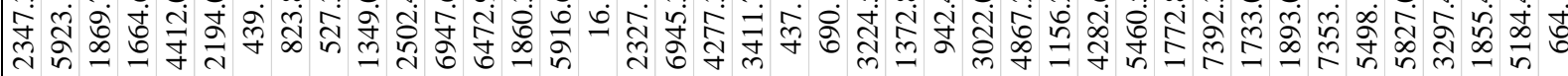

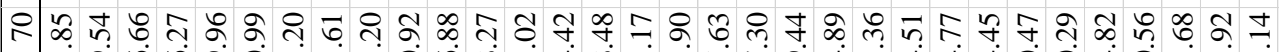

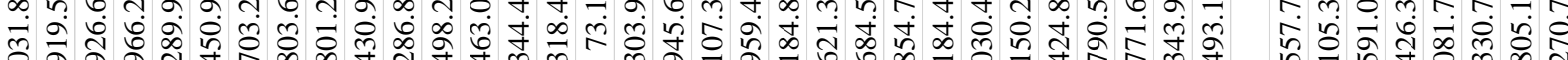

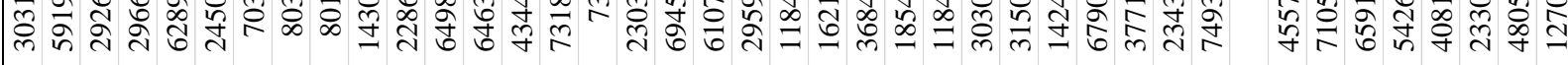

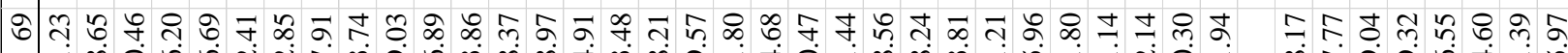

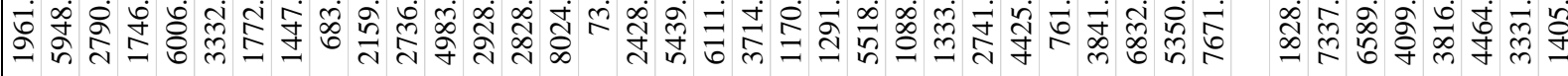

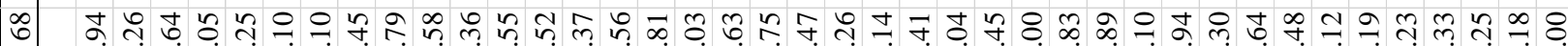

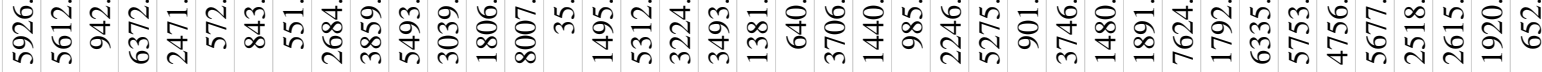

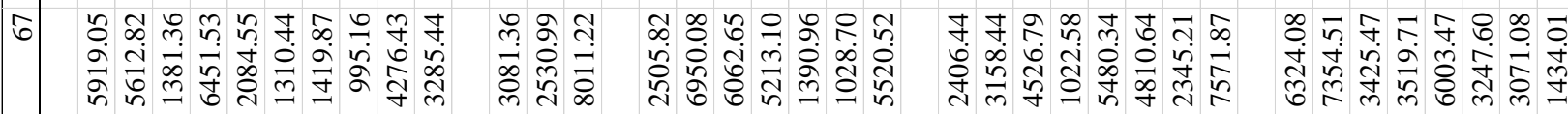

\& 수 等

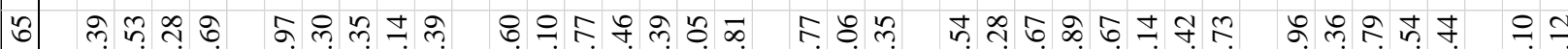

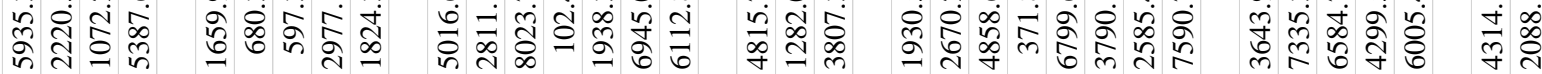

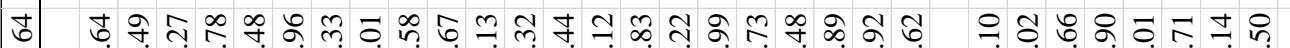

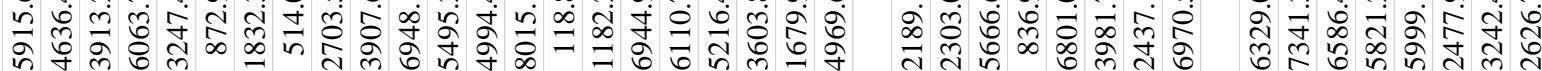




\section{Study 2}

\section{4}

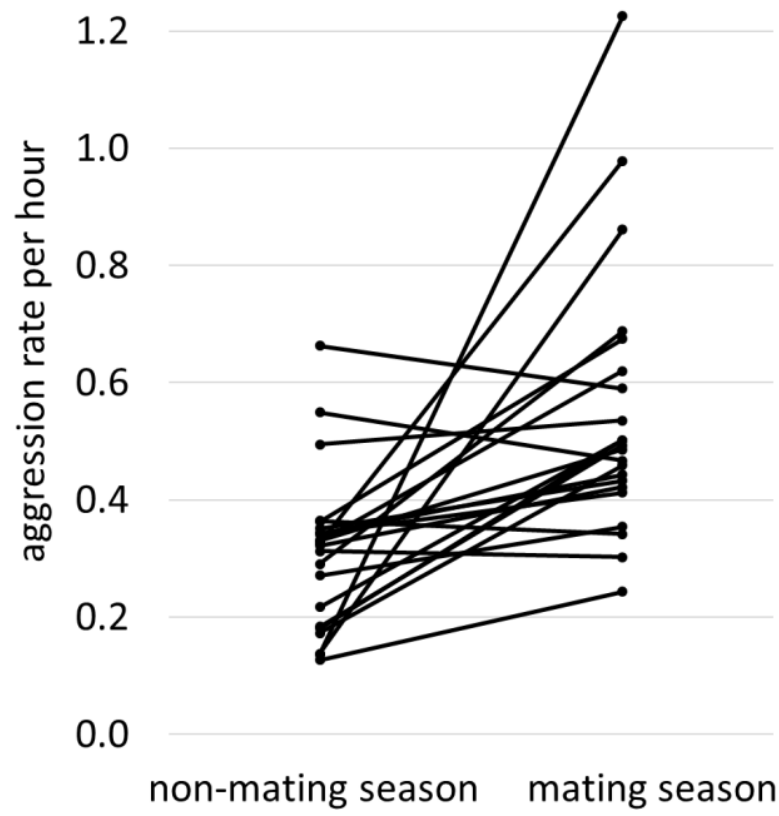

Figure S2.1: Change in aggression rate (per hour) of 22 adult male Assamese macaques from the nonmating to the mating season. Aggression rate is significantly higher during the mating season (Wilcoxon signed-rank test: $\mathrm{N}=22, \mathrm{p}<0.001, \mathrm{z}=-3.588$ ).

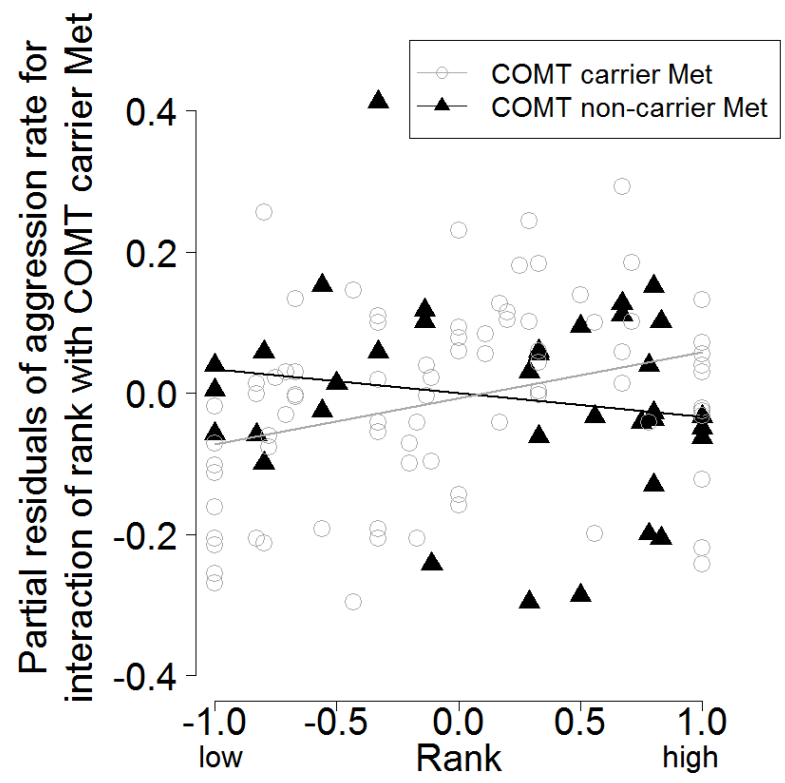

Figure S2.2: Partial residuals of the response variable aggression rate per hour for the interaction term of COMT carrier Met and rank in the aggression rate-model. 
Table S2.1: Full aggression rate-model with COMT non-carrier Met set as reference

\begin{tabular}{lrrrr}
\hline Fixed effect & Estimate & Std. Error & t-value & p-value \\
\hline Intercept & 0.341 & 0.033 & 10.331 & $<0.001$ \\
COMT carrier Met & -0.007 & 0.028 & -0.255 & 0.800 \\
Rank & -0.034 & 0.034 & -1.000 & 0.320 \\
Sex: male & 0.356 & 0.026 & 13.622 & $<0.001$ \\
Season: non-mating & -0.081 & 0.025 & -3.238 & 0.002 \\
Group: MST & -0.055 & 0.031 & -1.755 & 0.082 \\
Group: SOT & 0.013 & 0.037 & 0.346 & 0.730 \\
Group: SST & -0.046 & 0.039 & -1.189 & 0.237 \\
COMT carrier Met*rank & 0.099 & 0.041 & 2.397 & 0.018 \\
\hline
\end{tabular}

The same model diagnostics as outlined in the original paper were applied. A comparison of the full model to the null model revealed $\mathrm{p}=0.026 . \mathrm{R}^{2}$ of this model is 0.665. $\mathrm{N}=117$ data points for 59 individuals in two seasons

Table S2.2: Full aggression rate-model with COMT genotype Met/Met set as reference

\begin{tabular}{lrrrr}
\hline Fixed effect & Estimate & Std. Error & t-value & p-value \\
\hline Intercept & 0.347 & 0.042 & 8.259 & $<0.001$ \\
COMT genotype Met/Val & -0.017 & 0.036 & -0.478 & 0.633 \\
COMT genotype Val/Val & -0.005 & 0.039 & -0.127 & 0.899 \\
Rank & 0.082 & 0.046 & 1.786 & 0.077 \\
Sex: male & 0.353 & 0.027 & 13.147 & $<0.001$ \\
Season: non-mating & -0.080 & 0.025 & -3.190 & 0.002 \\
Group: MST & -0.055 & 0.032 & -1.726 & 0.087 \\
Group: SOT & 0.014 & 0.037 & 0.388 & 0.699 \\
Group: SST & -0.054 & 0.041 & -1.328 & 0.187 \\
COMT genotype Met/Val*rank & 0.026 & 0.055 & -0.471 & 0.638 \\
COMT genotype Val/Val*rank & -0.117 & 0.058 & -2.017 & 0.046 \\
\hline
\end{tabular}

$\mathrm{N}=117$ data points for 59 individuals in two seasons

Table S2.3: Full rank change-model of aggression (within individuals from the perspective of changing from a lower to a higher rank position) with COMT genotype Val/Met set as reference

\begin{tabular}{lrrrr}
\hline Effect & Estimate & Std. Error & t-value & $\mathrm{p}$-value \\
\hline Intercept & -0.240 & 0.139 & -1.725 & 0.105 \\
COMT genotype Met/Met & 0.245 & 0.158 & 1.554 & 0.141 \\
COMT genotype Val/Val & -0.132 & 0.150 & -0.883 & 0.391 \\
Season change: non-mating to mating & 0.496 & 0.122 & 4.065 & 0.001 \\
Group: MST & 0.217 & 0.151 & 1.437 & 0.171 \\
Group: SOT & 0.203 & 0.155 & 1.308 & 0.211 \\
Group: SST & 0.024 & 0.310 & 0.078 & 0.939 \\
\hline
\end{tabular}

$\mathrm{N}=22$ individuals 


\section{Study 3}

\section{Top three male partners}

We also calculated the maintenance- and the buffering-model considering males' top three strongest social bonds instead of males' top two strongest social bonds as in the main manuscript. The results are very similar to the models with top two partners and are shown in Table S3.1.

Table S3.1: Linear regression models considering males' top three strongest social bonds

\begin{tabular}{lrrrr}
\hline $\begin{array}{l}\text { (i) Maintenance-model with Hinde index as response. } \mathrm{n}=129 \text { data points } \\
\text { for } 23 \text { individuals and two seasons, significant difference to null model } \\
\mathrm{p}=0.003, \mathrm{R}^{2}=9.31 \%\end{array}$ \\
\hline Intercept & -3.632 & 4.731 & -0.768 & 0.444 \\
Genetic risk & 1.443 & 0.425 & 3.395 & 0.001 \\
Rank difference & -1.488 & 3.478 & -0.428 & 0.670 \\
Season: mating & -1.510 & 3.588 & -0.421 & 0.675 \\
\hline (ii) Buffering-model with log-transformed mean iUC levels as response. \\
n=41 data points for 22 individuals and two seasons, significant difference \\
to null model p=0.002, $\mathrm{R}^{2}=83.27 \%$ \\
\hline Intercept & 4.419 & 0.172 & 25.674 & $<0.001$ \\
Genetic risk & 0.310 & 0.105 & 2.954 & 0.009 \\
Sum of CSI & -0.310 & 0.099 & -3.132 & 0.004 \\
Dominance rank & -0.019 & 0.134 & -0.143 & 0.887 \\
Season: mating & 1.518 & 0.138 & 10.999 & $<0.001$ \\
Genetic risk:sum of CSI & 0.270 & 0.099 & 2.716 & 0.011 \\
\hline
\end{tabular}




\section{Dyadic composite sociality index (CSI)}

The CSI was calculated as follows:

$$
\operatorname{CSI}_{\mathrm{xy}}=\frac{\sum_{i=1}^{b}\left(\frac{f_{\mathrm{ixy}}}{f_{\mathrm{i}}}+\frac{d_{\mathrm{ixy}}}{d_{\mathrm{i}}}\right)}{2 b} \text {, where } \mathrm{b} \text { denotes the number of behaviors contributing to }
$$

the CSI, $f_{i x y}$ the frequency of behavior $i$ for the dyad $x y, f_{i}$ the mean frequency of behavior of all male-male dyads, $d_{i x y}$ the duration of behavior $i$ for the dyad $x y$ and $d_{i}$ the mean duration of behavior i for all male-male dyads. The average CSI is always one and the higher the value the stronger the relationship relative to the mean in the group at that time (Silk et al., 2006a). For the statistical analyses, we calculated the sum of a male's two strongest social bonds per reproductive season. The CSI ranged from 0.10 to 8.04 , the median was 0.74 and only $34.9 \%$ (90) of the dyads had a CSI above the average of 1.

\section{Hinde index}

The Hinde index was calculated as follows:

Hinde index $=100 \times\left(\frac{a_{\mathrm{x}}}{a_{\mathrm{x}}+a_{\mathrm{y}}}-\frac{d_{\mathrm{x}}}{d_{\mathrm{x}}+d_{\mathrm{y}}}\right)$, where $\mathrm{a}_{\mathrm{x}}$ denotes how often individual $\mathrm{x}$ approached individual $\mathrm{y}, \mathrm{a}_{\mathrm{y}}$ how often individual $\mathrm{y}$ approached individual $\mathrm{x}, \mathrm{d}_{\mathrm{x}}$ how often individual $\mathrm{x}$ departed individual $\mathrm{y}, \mathrm{d}_{\mathrm{y}}$ how often individual $\mathrm{y}$ departed individual $\mathrm{x}$. The Hinde index assesses the extent of symmetry in social interactions and ranges from -100 to 100 , with high values indicating that the individual $\mathrm{x}$ is mainly responsible for maintaining close proximity with individual y. For the statistical analyses, we included the two Hinde indices with the top two male partners for each reproductive season. 
Table S3.2: Dominance hierarchy characteristics

\begin{tabular}{|c|c|c|c|c|c|c|c|c|}
\hline season & & non- & ting & & & & & \\
\hline social group & ASM & ASS & AOM & AOS & ASM & ASS & $\mathrm{AOM}$ & AOS \\
\hline \# conflicts & 230 & 34 & 175 & 90 & 193 & 2 & 289 & 103 \\
\hline \# dyads & 36 & 3 & 21 & 6 & 21 & 1 & 21 & 21 \\
\hline \# unknown rel. & 4 & 0 & 1 & 0 & 1 & 0 & 0 & 3 \\
\hline \# two-way rel. & 4 & 0 & 2 & 2 & 2 & 0 & 4 & 1 \\
\hline winning & 0.80-1 & 1 & 0.83-1 & 0.83-1 & 0.88-1 & 1 & 0.88-1 & 0.9-1 \\
\hline proportion & (Ø 0.99$)$ & 1 & $(\varnothing 0.98)$ & $(\varnothing 0.96)$ & $(\varnothing 0.99)$ & 1 & $(\varnothing 0.99)$ & $(\varnothing 0.99)$ \\
\hline DCI & 0.90 & 1 & 0.98 & 0.93 & 0.98 & 1 & 0.96 & 0.98 \\
\hline
\end{tabular}

Note: \# conflicts =number of decided dyadic conflicts, rel. = relationships, DCI = directional consistency index (van Hooff \& Wensing, 1987), ASS in the mating season was excluded in the maintenance-, social bonding- and buffering-model in which males' top two or top three partners were considered 


\section{References}

Abbott, D. H., Keverne, E. B., Bercovitch, F. B., Shively, C. A., Mendoza, S. P., Saltzman, W., Snowdon, C. T., Ziegler, T. E., Banjevic, M., Garland, T., \& Sapolsky, R. M. (2003). Are subordinates always stressed? A comparative analysis of rank differences in cortisol levels among primates. Hormones and Behavior, $43(1), 67-82$.

Abegg, C., \& Thierry, B. (2002). Macaque evolution and dispersal in insular south-east Asia. Biological Journal of the Linnean Society, 75, 555-576.

Accorsi, P. A., Carloni, E., Valsecchi, P., Viggiani, R., Gamberoni, M., Tamanini, C., \& Seren, E. (2008). Cortisol determination in hair and faeces from domestic cats and dogs. General and Comparative Endocrinology, 155(2), 398-402.

Adrian, T. E., Allen, J. M., Bloom, S. R., Ghatei, M. A., Rossor, M. N., Roberts, G. W., Crow, T. J., Tatemoto, K., \& Polak, J. M. (1983). Neuropeptide Y distribution in human brain. Nature, 306(5943), 584-586.

Aggimarangsee, N. (1992). Survey for semi-tame colonies of macaques in Thailand. Natural History Bulletin of the Siam Society, 40,103-166.

Aguilera, G., \& Liu, Y. (2012). The molecular physiology of CRH neurons. Frontiers in Neuroendocrinology, 33(1), 67-84.

Alberts, S. C. (2010). Primatology: "a faithful friend is the medicine of life". Current Biology, 20(15), 632-634.

Altmann, S. A. (1965). Sociobiology of rhesus monkeys. II: Stochastics of social communication. Journal of Theoretical Biology, 8(3), 490-522.

Altmann, J., Alberts, S. C., Haines, S. A., Dubach, J., Muruthi, P., Coote, T., Geffen, E., Cheesman, D. J., Mututua, R. S., Saiyalel, S. N., Wayne, R. K., Lacy, R. C., \& Bruford, M. W. (1996). Behavior predicts genetic structure in a wild primate group. Proceedings of the National Academy of Sciences of the United States of America, 93(12), 5797-5801.

Altschul, S. F., Gish, W., Miller, W., Myers, E. W., \& Lipman, D. J. (1990). Basic local alignment search tool. Journal of Molecular Biology, 215(3), 403-410.

Altschul, S. F., Madden, T. L., Schäffer, A. A., Zhang, J., Zhang, Z., Miller, W., \& Lipman, D. J. (1997). Gapped BLAST and PSI-BLAST: a new generation of protein database search programs. Nucleic Acids Research, 25(17), 3389-3402.

Andrews, S. (2010) FastQC: a quality control tool for high throughput sequence data. Babraham Institute, www.bioinformatics.babraham.ac.uk 01.09.2017

Anestis, S. F. (2005). Behavioral style, dominance rank, and urinary cortisol in young chimpanzees (Pan troglodytes). Behaviour, 142, 1245-1268.

Anholt, R. R. H., \& Mackay, T. F. C. (2012). Genetics of aggression. Annual Review of Genetics, 46(1), 145-164.

Antoni, F. A., Holmes, M. C., Makara, G. B., Kárteszi, M., \& László, F. A. (1984). 
Evidence that the effects of arginine-8-vasopressin (AVP) on pituitary corticotropin (ACTH) release are mediated by a novel type of receptor. Peptides, 5(3), 519-522.

Archie, E. A., Tung, J., Clark, M., Altmann, J., \& Alberts, S. C. (2014). Social affiliation matters: both same-sex and opposite-sex relationships predict survival in wild female baboons. Proceedings of the Royal Society of London B: Biological Sciences, 281(1793), 20141261.

Armbruster, D., Mueller, A., Strobel, A., Lesch, K. P., Brocke, B., \& Kirschbaum, C. (2011). Predicting cortisol stress responses in older individuals: influence of serotonin receptor $1 \mathrm{~A}$ gene (HTR1A) and stressful life events. Hormones and Behavior, 60(1), 105-111.

Armbruster, D., Mueller, A., Strobel, A., Lesch, K. P., Brocke, B., \& Kirschbaum, C. (2012). Children under stress - COMT genotype and stressful life events predict cortisol increase in an acute social stress paradigm. The International Journal of Neuropsychopharmacology, 15(09), 1229-1239.

Auwera, G. A., Carneiro, M. O., Hartl, C., Poplin, R., del Angel, G., Levy-Moonshine, A., Jordan, T., Shakir, K., Roazen, D., \& Thibault, J. (2013). From FastQ data to high-confidence variant calls: the genome analysis toolkit best practices pipeline. Current Protocols in Bioinformatics, 43(10.11), 1-33.

Axelrod, J., \& Reisine, T. (1984). Stress hormones: their interaction and regulation. Science, 224(4648), 452-459.

Axelrod, J., \& Tomchick, R. (1958). Enzymatic O-methylation of epinephrine and other catechols. Journal of Biological Chemistry, 233(3), 702-705.

Bahr, N. I., Palme, R., Möhle, U., Hodges, J. K., Heistermann, M., Mo, U., Hodges, J. K., \& Heistermann, M. (2000). Comparative aspects of the metabolism and excretion of cortisol in three individual nonhuman primates. General and Comparative Endocrinology, 117(3), 427-438.

Baker, M. E., Chandsawangbhuwana, C., \& Ollikainen, N. (2007). Structural analysis of the evolution of steroid specificity in the mineralocorticoid and glucocorticoid receptors. BMC Evolutionary Biology, 7(1), 24.

Balasubramaniam, K., Beisner, B., Vandeleest, J., Atwill, E., \& McCowan, B. (2016). Social buffering and contact transmission: network connections have beneficial and detrimental effects on Shigella infection risk among captive rhesus macaques. PeerJ, 4, e2630.

Baldessarini, R. J., \& Hennen, J. (2004). Genetics of suicide: an overview. Harvard Review of Psychiatry, 12(1), 1-13.

Bale, T. L., Picetti, R., Contarino, A., Koob, G. F., Vale, W. W., \& Lee, K.-F. (2002). Mice deficient for both corticotropin-releasing factor receptor 1 (CRFR1) and CRFR2 have an impaired stress response and display sexually dichotomous anxietylike behavior. Journal of Neuroscience, 22(1), 193-199.

Balzarini, V., Taborsky, M., Wanner, S., Koch, F., \& Frommen, J. G. (2014). Mirror, mirror on the wall: the predictive value of mirror tests for measuring aggression in fish. Behavioral Ecology and Sociobiology, 68(5), 871-878.

Bamberger, C. M., Schulte, H. M., \& Chrousos, G. P. (1996). Molecular determinants of 
glucocorticoid receptor function and tissue sensitivity to glucocorticoids. Endocrine Reviews, 17(3), 245-261.

Barban, N., Jansen, R., De Vlaming, R., Vaez, A., Mandemakers, J. J., Tropf, F. C., Shen, X., Wilson, J. F., Chasman, D. I., \& Nolte, I. M. (2016). Genome-wide analysis identifies 12 loci influencing human reproductive behavior. Nature Genetics, 48(12), $1462-1472$.

Barlow, G. W., Rogers, W., \& Fraley, N. (1986). Do Midas cichlids win through prowess or daring? It depends. Behavioral Ecology and Sociobiology, 19(1), 1-8.

Barnes, J. C., TenEyck, M., Boutwell, B. B., \& Beaver, K. M. (2013). Indicators of domestic/intimate partner violence are structured by genetic and nonshared environmental influences. Journal of Psychiatric Research, 47(3), 371-376.

Baron, R., \& Richardson, D. (1994). Human aggression. New York: Plenum Press.

Barr, C. S. (2011). Non-human primate models of alcohol-related phenotypes: the influence of genetic and environmental factors. Current Topics in Behavioural Neuroscience, 13, 223-249.

Barr, C. S., Dvoskin, R. L., \& Yuan, Q. (2008). CRH haplotype as a factor influencing cerebrospinal fluid levels of corticotropin-releasing hormone, hypothalamicpituitary- adrenal axis activity, temperament and alcohol consumption in rhesus macaques. Archives of General Psychiatry, 65(8), 934-944.

Barr, C. S., Newman, T. K., Shannon, C., Parker, C., Dvoskin, R. L., Becker, M. L., Schwandt, M., Champoux, M., Lesch, K. P., Goldman, D., Suomi, S. J., \& Higley, J. D. (2004). Rearing condition and rh5-HTTLPR interact to influence limbichypothalamic-pituitary-adrenal axis response to stress in infant macaques. Biological Psychiatry, 55(7), 733-738.

Bartels, M., van den Berg, M., Sluyter, F., Boomsma, D. I., \& de Geus, E. J. C. (2003). Heritability of cortisol levels: review and simultaneous analysis of twin studies. Psychoneuroendocrinology, 28(2), 121-137.

Bates, D., Mächler, M., Bolker, B., \& Walker, S. (2015). Fitting linear mixed-effects models using lme4. Journal of Statistical Software, 67(1).

Baugh, A. T., Davidson, S. C., Hau, M., \& van Oers, K. (2017). Temporal dynamics of the HPA axis linked to exploratory behavior in a wild european songbird (Parus major). General and Comparative Endocrinology, 250, 104-112.

Beaver, K. M., Eagle Schutt, J., Boutwell, B. B., Ratchford, M., Roberts, K., \& Barnes, J. C. (2009). Genetic and environmental influences on levels of self-control and delinquent peer affiliation: results from a longitudinal sample of adolescent twins. Criminal Justice and Behavior, 36(1), 41-60.

Beehner, J. C., \& Bergman, T. J. (2017). The next step for stress research in primates: to identify relationships between glucocorticoid secretion and fitness. Hormones and Behavior, 91, 68-83.

Belsky, D. W., \& Israel, S. (2014). Integrating genetics and social science: genetic risk scores. Biodemography and Social Biology, 60(2), 137-155.

Belsky, J. (1997). Theory testing, effect-size evaluation, and differential susceptibility to 
rearing influence: the case of mothering and attachment. Child Development, 68(4), 598-600.

Belsky, J., Bakermans-Kranenburg, M. J., \& van IJzendoorn, M. H. (2007). For better and for worse: differential susceptibility to environmental influences. Current Directions in Psychological Science, 16(6), 300-304.

Belsky, J., \& Pluess, M. (2009a). Beyond diathesis stress: differential susceptibility to environmental influences. Psychological Bulletin, 135(6), 885-908.

Belsky, J., \& Pluess, M. (2009b). The nature (and nurture?) of plasticity in early human development. Perspectives on Psychological Science, 4(4), 345-351.

Benis, A. M., \& Hobgood, D. K. (2011). Dopamine receptor DRD3 codes for trait aggression as mendelian recessive. Medical Hypotheses, 77(6), 1108-1110.

Bennett, A. J., Lesch, K. P., Heils, A., Long, J. C., Lorenz, J. G., Shoaf, S. E., Champoux, M., Suomi, S. J., Linnoila, M. V., \& Higley, J. D. (2002). Early experience and serotonin transporter gene variation interact to influence primate CNS function. Molecular Psychiatry, 7(1), 118-122.

Bergey, C. M., Phillips-Conroy, J. E., Disotell, T. R., \& Jolly, C. J. (2016). Dopamine pathway is highly diverged in primate species that differ markedly in social behavior. Proceedings of the National Academy of Sciences of the United States of America, 113(22), 6178-6181.

Berghänel, A., Heistermann, M., Schülke, O., \& Ostner, J. (2016). Prenatal stress effects in a wild, long-lived primate: predictive adaptive responses in an unpredictable environment. Proceedings of the Royal Society B: Biological Sciences, 283(1839), 20161304.

Berghänel, A., Ostner, J., Schröder, U., \& Schülke, O. (2011). Social bonds predict future cooperation in male Barbary macaques, Macaca sylvanus. Animal Behaviour, 81(6), 1109-1116.

Berghänel, A., Schülke, O., \& Ostner, J. (2015). Locomotor play drives motor skill acquisition at the expense of growth: a life history trade-off. Science Advances, 1(7), e1500451.

Berkman, L. F., Glass, T., Brissette, I., \& Seeman, T. E. (2000). From social integration to health: Durkheim in the new millennium. Social Science \& Medicine, 51(6), 843857.

Berkman, L. F., \& Syme, S. L. (1979). Social networks, host resistance, and mortality: a nine-year follow-up study of alameda county residents. American Journal of Epidemiology, 109(2), 186-204.

Bernstein, I. S., \& Cooper, M. A. (1999). Dominance in Assamese macaques (Macaca assamensis). American Journal of Primatology, 48(4), 283-289.

Bhakta, S. G., Zhang, J.-P., \& Malhotra, A. K. (2012). The COMT Met158 allele and violence in schizophrenia: a meta-analysis. Schizophrenia Research, 140(1), 192197.

Binder, E. B., Bradley, R. G., Liu, W., Epstein, M. P., Deveau, T. C., Mercer, K. B., Tang, Y., Gillespie, C. F., Heim, C. M., \& Nemeroff, C. B. (2008). Association of 
FKBP5 polymorphisms and childhood abuse with risk of posttraumatic stress disorder symptoms in adults. Jama, 299(11), 1291-1305.

Binder, E. B., \& Nemeroff, C. B. (2010). The CRF system, stress, depression and anxietyinsights from human genetic studies. Molecular Psychiatry, 15(6), 574-88.

Bolger, A. M., Lohse, M., \& Usadel, B. (2014). Trimmomatic: a flexible trimmer for illumina sequence data. Bioinformatics, 30(15), 2114-2120.

Bolton, J. L., Hayward, C., Direk, N., Lewis, J. G., Hammond, G. L., Hill, L. A., Anderson, A., Huffman, J., Wilson, J. F., \& Campbell, H. (2014). Genome wide association identifies common variants at the SERPINA6/SERPINA1 locus influencing plasma cortisol and corticosteroid binding globulin. PLoS Genetics, 10(7), e1004474.

Borries, C., Larney, E., Derby, A. M., \& Koenig, A. (2004). Temporary absence and dispersal in Phayre's leaf monkeys (Trachypithecus phayrei). Folia Primatologica, 75(1), 27-30.

Borries, C., Larney, E., Kreetiyutanont, K., \& Koenig, A. (2002). The diurnal primate community in a dry evergreen forest in Phu Khieo Wildlife Sanctuary, Northeast Thailand. Natural History Bulletin of the Siam Society, 50(1), 75-88.

Bouma, E. M. C., Riese, H., Doornbos, B., Ormel, J., \& Oldehinkel, A. J. (2012). Genetically based reduced MAOA and COMT functioning is associated with the cortisol stress response: a replication study. Molecular Psychiatry, 17(2), 119-121.

Brandon-Jones, D., Eudey, A. A., Geissmann, T., Groves, C. P., \& Melnick, D. J. (2004). Asian primate classification. International Journal of Primatology, 25(1), 97-164.

Brendgen, M., Vitaro, F., Boivin, M., Dionne, G., \& Pérusse, D. (2006). Examining genetic and environmental effects on reactive versus proactive aggression. Developmental Psychology, 42(6), 1299-1312.

Brennan, P. A., Hammen, C., Sylvers, P., Bor, W., Najman, J., Lind, P., Montgomery, G., \& Smith, A. K. (2011). Interactions between the COMT Val108/158Met polymorphism and maternal prenatal smoking predict aggressive behavior outcomes. Biological Psychology, 87(1), 99-105.

Brent, D. A., \& Mann, J. J. (2005). Family genetic studies, suicide, and suicidal behavior. American Journal of Medical Genetics Part C: Seminars in Medical Genetics, 133, 13-24.

Brent, L. J. N., Heilbronner, S. R., Horvath, J. E., Gonzalez-Martinez, J., Ruiz-Lambides, A., Robinson, A. G., Skene, J. H. P., \& Platt, M. L. (2013). Genetic origins of social networks in rhesus macaques. Scientific Reports, 3, 1042.

Brent, L. J. N., \& Melin, A. D. (2014). The genetic basis of primate behavior: genetics and genomics in field-based primatology. International Journal of Primatology, $35(1), 1-10$.

Brent, L. J. N., Semple, S., Maclarnon, A., Ruiz-lambides, A., Gonzalez-martinez, J., \& Platt, M. L. (2014). Personality traits in rhesus macaques (Macaca mulatta) are heritable but do not predict reproductive output. International Journal of Primatology, 35(1),188-209. 
Brothers, S. P., \& Wahlestedt, C. (2010). Therapeutic potential of neuropeptide Y (NPY) receptor ligands. EMBO Molecular Medicine, 2(11), 429-439.

Brown, A. B., Biederman, J., Valera, E. M., Doyle, A. E., Bush, G., Spencer, T., Monuteaux, M. C., Mick, E., Whitfield-Gabrieli, S., Makris, N., Whitfield-Gabrieli, S., Makris, N., LaViolette, P. S., Oscar-Berman, M., Faraone, S. V., \& Seidman, L. J. (2010). Effect of dopamine transporter gene (SLC6A3) variation on dorsal anterior cingulate function in attention-deficit/hyperactivity disorder. American Journal of Medical Genetics, Part B: Neuropsychiatric Genetics, 153(2), 365-375.

Brown, J. R., Ye, H., Bronson, R. T., Dikkes, P., \& Greenberg, M. E. (1996). A defect in nurturing in mice lacking the immediate early gene fosB. Cell, 86(2), 297-309.

Bruder, D., Westendorf, A. M., Geffers, R., Gruber, A. D., Gereke, M., Enelow, R. I., \& Buer, J. (2004). CD4 T lymphocyte-mediated lung disease: steady state between pathological and tolerogenic immune reactions. American Journal of Respiratory and Critical Care Medicine, 170(11), 1145-1152.

Brunner, H. G., Nelen, M. R., van Zandvoort, P., Abeling, N. G., van Gennip, A. H., Wolters, E. C., Kuiper, M. A., Ropers, H. H. \& van Oost, B. A. (1993). X-linked borderline mental retardation with prominent behavioral disturbance: phenotype, genetic localization, and evidence for disturbed monoamine metabolism. American Journal of Human Genetics, 52(6), 1032-1039.

Buckholtz, J. W., \& Meyer-Lindenberg, A. (2008). MAOA and the neurogenetic architecture of human aggression. Trends in Neurosciences, 31(3), 120-129.

Buss, A. H., \& Durkee, A. (1957). An inventory for assessing different kinds of hostility. Journal of Consulting Psychology, 21(4), 343-349.

Buss, A. H., \& Perry, M. (1992). The aggression questionnaire. Journal of Personality and Social Psychology, 63(3), 452-459.

Buss, D. M., \& Duntley, J. D. (2006). The evolution of aggression. In Schaller, M., Simpson J. A., \& Kenrick, D. T. (Eds.), Evolution and social psychology, 263-286. New York: Psychology Press.

Byrne, R. W. (2007). Culture in great apes: using intricate complexity in feeding skills to trace the evolutionary origin of human technical prowess. Philosophical Transactions of the Royal Society of London B: Biological Sciences, 362(1480), 577-585.

Cacioppo, J. T., \& Cacioppo, S. (2014). Social relationships and health: the toxic effects of perceived social isolation. Social and Personality Psychology Compass, 8(2), 5872 .

Cacioppo, J. T., Cacioppo, S., Capitanio, J. P., \& Cole, S. W. (2015). The neuroendocrinology of social isolation. Annual Review of Psychology, 66, 733-767.

Cagliani, R., Fumagalli, M., Riva, S., Pozzoli, U., Comi, G. P., Menozzi, G., Bresolin, N., $\&$ Sironi, M. (2008). The signature of long-standing balancing selection at the human defensin $\beta-1$ promoter. Genome Biology, 9(9), R143.

Cagliani, R., Fumagalli, M., Biasin, M., Piacentini, L., Riva, S., Pozzoli, U., Bonaglia, M. C., Bresolin, N., Clerici, M., \& Sironi, M. (2010). Long-term balancing selection maintains trans-specific polymorphisms in the human TRIM5 gene. Human 
Genetics, 128(6), 577-588.

Cagliani, R., Guerini, F. R., Fumagalli, M., Riva, S., Agliardi, C., Galimberti, D., Pozzoli, U., Goris, A., Dubois, B., \& Fenoglio, C. (2012). A trans-specific polymorphism in ZC3HAV1 is maintained by long-standing balancing selection and may confer susceptibility to multiple sclerosis. Molecular Biology and Evolution, 29(6), 15991613.

Caldwell, H. K., \& Young III, W. S. (2006). Oxytocin and vasopressin: genetics and behavioral implications. In Lajtha A., \& Lim, R. (Eds.), Handbook of Neurochemistry and Molecular Neurobiology, 573-607. Boston: Springer.

Campbell, L. A. D., Tkaczynski, P. J., Lehmann, J., Mouna, M., \& Majolo, B. (2018). Social thermoregulation as a potential mechanism linking sociality and fitness: Barbary macaques with more social partners form larger huddles. Scientific Reports, 8, 6074 .

Carere, C., Groothuis, T. G. G., Möstl, E., Daan, S., \& Koolhaas, J. M. (2003). Fecal corticosteroids in a territorial bird selected for different personalities: daily rhythm and the response to social stress. Hormones and Behavior, 43(5), 540-548.

Carpenter, C. R. (1945). Concepts and problems of primate sociometry. Sociometry, 8(1), $56-61$.

Carroll, B. J., Curtis, G. C., \& Mendels, J. (1976). Neuroendocrine regulation in depression: II. Discrimination of depressed from nondepressed patients. Archives of General Psychiatry, 33(9), 1051-1058.

Carter, A. J., Macdonald, S. L., Thomson, V. A., \& Goldizen, A. W. (2009). Structured association patterns and their energetic benefits in female eastern grey kangaroos, Macropus giganteus. Animal Behaviour, 77(4), 839-846.

Carter, K. D., Brand, R., Carter, J. K., Shorrocks, B., \& Goldizen, A. W. (2013). Social networks, long-term associations and age-related sociability of wild giraffes. Animal Behaviour, 86(5), 901-910.

Carter, K. D., Seddon, J. M., Frère, C. H., Carter, J. K., \& Goldizen, A. W. (2013). Fission-fusion dynamics in wild giraffes may be driven by kinship, spatial overlap and individual social preferences. Animal Behaviour, 85(2), 385-394.

Cases, O., Seif, I., Grimsby, J., Gaspar, P., Chen, K., Pournin, S., Müller, U., Aguet, M., Babinet, C., \& Shih, J. C. (1995). Aggressive behavior and altered amounts of brain serotonin and norepinephrine in mice lacking MAOA. Science, 268(5218), 17631766.

Caspi, A., Sugden, K., Moffitt, T. E., Taylor, A., Craig, I. W., Harrington, H., McClay, J., Mill, J., Martin, J., \& Braithwaite, A. (2003). Influence of life stress on depression: moderation by a polymorphism in the 5-HTT gene. Science, 301(5631), 386-389.

Cavigelli, S. (1999). Behavioural patterns associated with faecal cortisol levels in freeranging female ring-tailed lemurs, Lemur catta. Animal Behaviour, 57(4), 935-944.

Cavigelli, S., \& Caruso, M. J. (2015). Sex, social status and physiological stress in primates: the importance of social and glucocorticoid dynamics. Philosophical Transactions of the Royal Society B: Biological Sciences, 370, 20140103. 
Chakraborty, S., Chakraborty, D., Mukherjee, O., Jain, S., Ramakrishnan, U., \& Sinha, A. (2010). Genetic polymorphism in the serotonin transporter promoter region and ecological success in macaques. Behavior Genetics, 40(5), 672-679.

Chalise, M. K. (1999). Some behavioral and ecological aspects of Assamese monkeys (Macaca assamensis) in Makalu-Barun area, Nepal. Nepal Journal of Science and Technology, 1, 85-90.

Chalise, M. K. (2003). Assamese macaques (Macaca assamensis) in Nepal. Primate Conservation, 19, 99-107.

Chapais, B. (1995). Alliances as a means of competition in primates: evolutionary, developmental, and cognitive aspects. American Journal of Physical Anthropology, $38(21), 115-136$.

Chapais, B. (2004). How kinship generates dominance structures: a comparative perspective. In Thierry. B., Singh, M., \& Kaumanns, W. (Eds.), Macaque societies: A model for the study of social organization, 186-203. Cambridge: Cambridge University Press.

Charlesworth, D. (2006). Balancing selection and its effects on sequences in nearby genome regions. PLoS Genetics, 2(4), e64.

Charmandari, E., Tsigos, C., \& Chrousos, G. (2005). Endocrinology of the stress response. Annual Review of Physiology, 67, 259-284.

Charpentier, M. J. E., Fontaine, M. C., Cherel, E., Renoult, J. P., Jenkins, T., Benoit, L., Barthes, N., Alberts, S. C., \& Tung, J. (2012). Genetic structure in a dynamic baboon hybrid zone corroborates behavioural observations in a hybrid population. Molecular Ecology, 21(3), 715-731.

Chen, J., Lipska, B. K., Halim, N., Ma, Q. D., Matsumoto, M., Melhem, S., Kolachana, B. S., Hyde, T. M., Herman, M. M., \& Apud, J. (2004). Functional analysis of genetic variation in catechol-O-methyltransferase (COMT): effects on mRNA, protein, and enzyme activity in postmortem human brain. The American Journal of Human Genetics, 75(5), 807-821.

Chen, G. L., Novak, M. A., Hakim, S., Xie, Z., \& Miller, G. M. (2006). Tryptophan hydroxylase-2 gene polymorphisms in rhesus monkeys: association with hypothalamic-pituitary-adrenal axis function and in vitro gene expression. Molecular Psychiatry, 11(10), 914-918.

Chen, G. L., Novak, M. A., Meyer, J. S., Kelly, B. J., Vallender, E. J., \& Miller, G. M. (2010). TPH2 5'-and 3'-regulatory polymorphisms are differentially associated with HPA axis function and self-injurious behavior in rhesus monkeys. Genes, Brain and Behavior, 9(3), 335-347.

Chen, F. S., Kumsta, R., von Dawans, B., Monakhov, M., Ebstein, R. P., \& Heinrichs, M. (2011a). Common oxytocin receptor gene (OXTR) polymorphism and social support interact to reduce stress in humans. Proceedings of the National Academy of Sciences of the United States of America, 108(50), 19937-42.

Chen, H., Poon, A., Yeung, C., Helms, C., Pons, J., Bowcock, A. M., Kwok, P.-Y., \& Liao, W. (2011b). A genetic risk score combining ten psoriasis risk loci improves disease prediction. PLOS ONE, 6(4), e19454. 
Chen, G. L., \& Miller, G. M. (2012). Advances in tryptophan hydroxylase-2 gene expression regulation: new insights into serotonin-stress interaction and clinical implications. American Journal of Medical Genetics, Part B: Neuropsychiatric Genetics, 159(2), 152-171.

Cheney, D. L., \& Seyfarth, R. M. (2009). Stress and coping mechanisms in female primates. Advances in the Study of Behavior, 39, 1-44.

Cheney, D. L., Silk, J. B., \& Seyfarth, R. M. (2016). Network connections, dyadic bonds and fitness in wild female baboons. Royal Society Open Science, 3(7), 160255.

Chinwalla, A. T., Cook, L. L., Delehaunty, K. D., Fewell, G. A., Fulton, L. A., Fulton, R. S., Graves, T. A., Hillier, LaD. W., Mardis, E. R., \& McPherson, J. D. (2002). Initial sequencing and comparative analysis of the mouse genome. Nature, 420, 520-562.

Cingolani, P., Platts, A., Wang, L. L. L., Coon, M., Nguyen, T., Wang, L. L. L., Land, S. J., Lu, X., \& Ruden, D. M. (2012). A program for annotating and predicting the effects of single nucleotide polymorphisms, SnpEff: SNPs in the genome of Drosophila melanogaster strain w1118; iso-2; iso-3. Fly, 6(2), 80-92.

Clark, A. J. L., Grossman, A., \& McLoughlin, L. (1993). Familial glucocorticoid deficiency associated with point mutation in the adrenocorticotropin receptor. The Lancet, 341(8843), 461-462.

Coccaro, E. F., Lawrence, T., Trestman, R., Gabriel, S., Klar, H. M., \& Siever, L. J. (1991). Growth hormone responses to intravenous clonidine challenge correlate with behavioral irritability in psychiatric patients and healthy volunteers. Psychiatry Research, 39(2), 129-139.

Cohen, S., \& McKay, G. (1984). Social support, stress, and the buffering hypothesis: a theoretical analysis. In Baum, A., Taylor, S. E., \& Singer, J. E. (Eds.), Handbook of Psychology and Health, 253-267. Hillsdale: Lawrence Erlbaum Associates Publishers.

Cohen, S., \& Wills, T. A. (1985). Stress, social support, and the buffering hypothesis. Psychological Bulletin, 98(2), 310-357.

Cohen, S. (1988). Psychosocial models of the role of social support in the etiology of physical disease. Health Psychology, 7(3), 269-297.

Cohen, S., Janicki-Deverts, D., \& Miller, G. E. (2007). Psychological stress and disease. JAMA, 298(14), 1685-1687.

Cole, S. W., Mendoza, S. P., \& Capitanio, J. P. (2009). Social stress desensitizes lymphocytes to regulation by endogenous glucocorticoids: insights from in vivo cell trafficking dynamics in rhesus macaques. Psychosomatic Medicine, 71(6), 591-597.

Cole, S. W., Capitanio, J. P., Chun, K., Arevalo, J. M. G., Ma, J., \& Cacioppo, J. T. (2015). Myeloid differentiation architecture of leukocyte transcriptome dynamics in perceived social isolation. Proceedings of the National Academy of Sciences of the United States of America, 112(49), 15142-15147.

Conner, T. S., Jensen, K. P., Tennen, H., Furneaux, H. M., Kranzler, H. R., \& Covault, J. (2010). Functional polymorphisms in the serotonin 1B receptor gene (HTR1B) predict self-reported anger and hostility among young men. American Journal of Medical Genetics Part B: Neuropsychiatric Genetics, 153(1), 67-78. 
Connor, R. C. (2000). Group living in whales and dolphins. In Mann, J., Connor, R. C., Tyack, P. L., \& Whitehead, H. (Eds.), Cetacean Societies: field studies of dolphins and whales, 199-218. Chicago: University of Chicago Press.

International Human Genome Sequencing Consortium. (2001). Initial sequencing and analysis of the human genome. Nature, 409(6822), 860-921.

Conway, C. C., Keenan-Miller, D., Hammen, C., Lind, P. A., Najman, J. M., \& Brennan, P. A. (2012). Coaction of stress and serotonin transporter genotype in predicting aggression at the transition to adulthood. Journal of Clinical Child \& Adolescent Psychology, 41(1), 53-63.

Cooper, M. A., \& Bernstein, I. S. (2000). Social grooming in Assamese macaques (Macaca assamensis). American Journal of Primatology, 50(1), 77-85.

Cooper, M. A., \& Bernstein, I. S. (2008). Evaluating dominance styles in Assamese and rhesus macaques. International Journal of Primatology, 29(1), 225-243.

Cooper, M. A., Bernstein, I. S., \& Hemelrijk, C. K. (2005). Reconciliation and relationship quality in Assamese macaques (Macaca assamensis). American Journal of Primatology, 65(3), 269-282.

Cords, M. (1997). Friendships, alliances, reciprocity and repair. In Whiten, A. \& Byrne, R. W. (Eds.), Machiavellian intelligence II: extensions and evaluations, 24-49. Cambridge: Cambridge University Press.

Costas, J., Sanjuán, J., Ramos-Ríos, R., Paz, E., Agra, S., Ivorra, J. L., Páramo, M., Brenlla, J., \& Arrojo, M. (2011). Heterozygosity at catechol-O-methyltransferase Val158Met and schizophrenia: new data and meta-analysis. Journal of Psychiatric Research, 45(1), 7-14.

Craig, I. W. (2007). The importance of stress and genetic variation in human aggression. BioEssays, 29(3), 227-236.

Craig, I. W., \& Halton, K. E. (2009). Genetics of human aggressive behaviour. Human Genetics, 126(1), 101-113.

Crockford, C., Wittig, R. M., Whitten, P. L., Seyfarth, R. M., \& Cheney, D. L. (2008). Social stressors and coping mechanisms in wild female baboons (Papio hamadryas ursinus), Hormones and Behavior, 53(1), 254-265.

Crockford, C., Deschner, T., \& Wittig, R. M. (2017). The role of oxytocin in social buffering: what do primate studies add? Current Topics in Behavioural Neuroscience, 35, 155-173.

Curran, K. P., \& Chalasani, S. H. (2012). Serotonin circuits and anxiety: what can invertebrates teach us? Invertebrate Neuroscience, 12(2), 81-92.

D'souza, U. M., \& Craig, I. W. (2006). Functional polymorphisms in dopamine and serotonin pathway genes. Human Mutation, 27(1), 1-13.

Dambacher, F., Sack, A. T., Lobbestael, J., Arntz, A., Brugman, S., \& Schuhmann, T. (2014). Out of control: evidence for anterior insula involvement in motor impulsivity and reactive aggression. Social Cognitive and Affective Neuroscience, 10(4), 508516. 
Dambacher, F., Schuhmann, T., Lobbestael, J., Arntz, A., Brugman, S., \& Sack, A. T. (2015). Reducing proactive aggression through non-invasive brain stimulation. Social Cognitive and Affective Neuroscience, 10(10), 1303-1309.

Danish, L. M., Heistermann, M., Agil, M., \& Engelhardt, A. (2015). Validation of a novel collection device for non-invasive urine sampling from free-ranging animals, PLOS ONE, 10(11), 1-13.

Dathe, H. (1983). Haltungsrekord eines weiblichen Bartrhesus (Macaca assamensis McClelland, 1839) im Tierpark Berlin. Zoologischer Garten, 53, 125-132.

de Almeida, R. M. M., Ferrari, P. F., Parmigiani, S., \& Miczek, K. A. (2005). Escalated aggressive behavior: dopamine, serotonin and GABA. European Journal of Pharmacology, 526(1), 51-64.

de Boer, S. F., \& Koolhaas, J. M. (2005). 5-HT 1A and 5-HT 1B receptor agonists and aggression: a pharmacological challenge of the serotonin deficiency hypothesis. European Journal of Pharmacology, 526(1), 125-139.

de Kloet, E. R., Joëls, M., \& Holsboer, F. (2005). Stress and the brain: from adaptation to disease. Nature Reviews Neuroscience, 6(6), 463-475.

de Waal, F. B. M. (1992). Aggression as a well-integrated part of primate social relationships: a critique of the seville statement on violence. In Silverberg, J., \& Gray, J. P. (Eds.), Aggression and peacefulness in humans and other primates, 3756. Oxford: Oxford University Press.

de Waal, F. B. M. (2009). Tree of origin: what primate behavior can tell us about human social evolution. Harvard: Harvard University Press.

Deag, J. M. (1977). Aggression and submission in monkey societies. Animal Behaviour, 25(2), 465-474.

Del Rey, A., Chrousos, G., \& Besedovsky, H. (2008). The hypothalamus-pituitary adrenal axis. Amsterdam: Elsevier.

Delson, E. (1980). Fossil macaques, phyletic relationships and a scenario of deployment. In Lindburg, D. G. (Ed.), The macaques: studies in ecology, behavior and evolution, 10-30. New York: Van Nostrand Reinhold.

Denver, R. J. (2009). Structural and functional evolution of vertebrate neuroendocrine stress systems. Annals of the New York Academy of Sciences, 1163(1), 1-16.

Di Fiore, A. (2003). Molecular genetic approaches to the study of primate behavior, social organization, and reproduction. American Journal of Physical Anthropology, 122(46), 62-99.

Di Iorio, C. R., Carey, C. E., Michalski, L. J., Corral-Frias, N. S., Conley, E. D., Hariri, A. R., \& Bogdan, R. (2017). Hypothalamic-pituitary-adrenal axis genetic variation and early stress moderates amygdala function. Psychoneuroendocrinology, 80, 170 178.

Dickinson, D., \& Elvevåg, B. (2009). Genes, cognition and brain through a COMT lens. Neuroscience, 164(1), 72-87.

Ditzen, B., Neumann, I. D., Bodenmann, G., von Dawans, B., Turner, R. A., Ehlert, U., \& 
Heinrichs, M. (2007). Effects of different kinds of couple interaction on cortisol and heart rate responses to stress in women. Psychoneuroendocrinology, 32(5), 565-574.

Ditzen, B., Schmidt, S., Strauss, B., Nater, U. M., Ehlert, U., \& Heinrichs, M. (2008). Adult attachment and social support interact to reduce psychological but not cortisol responses to stress. Journal of Psychosomatic Research, 64(5), 479-486.

Dobson, S. D., \& Brent, L. J. N. (2013). On the evolution of the serotonin transporter linked polymorphic region (5-HTTLPR) in primates. Frontiers in Human Neuroscience, 7, 588.

Donaldson, Z. R., \& Young, L. J. (2008). Oxytocin, vasopressin, and the neurogenetics of sociality. Science, 322(5903), 900-904.

Drabant, E. M., Hariri, A. R., Meyer-Lindenberg, A., Munoz, K. E., Mattay, V. S., Kolachana, B. S., Egan, M. F., \& Weinberger, D. R. (2006). Catechol Omethyltransferase val158met genotype and neural mechanisms related to affective arousal and regulation. Archives of General Psychiatry, 63(12), 1396-1406.

Drouin, J. (2011). Chapter 1 - Pituitary development. In Melmed, S. (Ed.), The Pituitary, 3-19. San Diego: Academic Press.

Duman, R. S., \& Monteggia, L. M. (2006). A neurotrophic model for stress-related mood disorders. Biological Psychiatry, 59(12), 1116-1127.

Dunbar, R. I. M. (1991). Functional significance of social grooming in primates. Folia Primatologica, 57(3), 121-131.

Dunbar, R. I. M. (2012). Bridging the bonding gap: the transition from primates to humans. Philosophical Transactions of the Royal Society of London B: Biological Sciences, 367(1597), 1837-1846.

Dunbar, R. I. M. (2018). Social structure as a strategy to mitigate the costs of group living: a comparison of gelada and guereza monkeys. Animal Behaviour, 136, 5364.

Eaton, G. G., Modahl, K. B., \& Johnson, D. F. (1981). Aggressive behavior in a confined troop of Japanese macaques: effects of density, season, and gender. Aggressive Behavior, 7(2), 145-164.

Ebbert, M. T. W., Wadsworth, M. E., Staley, L. A., Hoyt, K. L., Pickett, B., Miller, J., Duce, J., Kauwe, J. S. K., \& Ridge, P. G. (2016). Evaluating the necessity of PCR duplicate removal from next-generation sequencing data and a comparison of approaches. BMC Bioinformatics, 17(7), 239.

Ebstein, R. P., Israel, S., Chew, S. H., Zhong, S., \& Knafo, A. (2010). Genetics of human social behavior. Neuron, 65(6), 831-844.

Edes, A. N., \& Crews, D. E. (2017). Allostatic load and biological anthropology. American Journal of Physical Anthropology, 162(63), 44-70.

Edgar, J., Held, S., Paul, E., Pettersson, I., Price, R. I., \& Nicol, C. (2015). Social buffering in a bird. Animal Behaviour, 105, 11-19.

Ekblom, R., \& Galindo, J. (2011). Applications of next generation sequencing in molecular ecology of non-model organisms. Heredity, 107(1), 1-15. 
Elenkov, I. J., \& Chrousos, G. P. (1999). Stress hormones, Th1/Th2 patterns, pro/antiinflammatory cytokines and susceptibility to disease. Trends in Endocrinology \& Metabolism, 10(9), 359-368.

Elsea, S. H., \& Lucas, R. E. (2002). The mousetrap: what we can learn when the mouse model does not mimic the human disease. ILAR Journal, 43(2), 66-79.

Enman, N. M., Sabban, E. L., McGonigle, P., \& van Bockstaele, E. J. (2015). Targeting the neuropeptide Y system in stress-related psychiatric disorders. Neurobiology of Stress, 1(1), 33-43.

Enoch, M. A., Hodgkinson, C. A., Gorodetsky, E., Goldman, D., \& Roy, A. (2013). Independent effects of 5' and 3' functional variants in the serotonin transporter gene on suicidal behavior in the context of childhood trauma. Journal of Psychiatric Research, 47(7), 900-907.

Eppig, J. T., Motenko, H., Richardson, J. E., Richards-Smith, B., \& Smith, C. L. (2015). The international mouse strain resource (IMSR): cataloging worldwide mouse and ES cell line resources. Mammalian Genome, 26(9-10), 448-455.

Eudey, A. A. (1980). Pleistocene glacial phenomena and the evolution of Asia macaques. In Lindburg, D. (Ed.), The macaques: Studies in ecology, behavior and evolution, 52-83. New York: Van Nostrand Reinhold.

Eudey, A. A. (2013). Assamese macaque Macaca assamensis. In Mittermeier, R. A., Rylands, A. B., \& Wilson, D. E. (Eds.), Handbook of the Mammals of the WorldVolume 3 Primates, 641-642. Barcelona: Lynx Edicions.

Evans, D. M., Visscher, P. M., \& Wray, N. R. (2009). Harnessing the information contained within genome-wide association studies to improve individual prediction of complex disease risk. Human Molecular Genetics, 18(18), 3525-3531.

Everly, G. S., \& Lating, J. M. (2013). The anatomy and physiology of the human stress response. In Everly, G. S. \& Lating, J. M. (Eds.), A clinical guide to the treatment of the human stress response, 17-51. New York: Springer.

Ewing, B., Hillier, L., Wendl, M. C., \& Green, P. (1998). Base-calling of automated sequencer traces using Phred. I. Accuracy assessment. Genome Research, 8(3), 175185.

Ezran, C., Karanewsky, C. J., Pendleton, J. L., Sholtz, A., Krasnow, M. R., Willick, J., Razafindrakoto, A., Zohdy, S., Albertelli, M. A., \& Krasnow, M. A. (2017). The mouse lemur, a genetic model organism for primate biology, behavior, and health. Genetics, 206(2), 651-664.

Fa, J. E. (1989). The genus Macaca: a review of taxonomy and evolution. Mammal Review, 19(2), 45-81.

Fairbanks, L. A., Jorgensen, M. J., Bailey, J. N., Breidenthal, S. E., Grzywa, R., \& Laudenslager, M. L. (2011). Heritability and genetic correlation of hair cortisol in vervet monkeys in low and higher stress environments. Psychoneuroendocrinology, 36(8), 1201-1208.

Fairbanks, L. A., Newman, T. K., Bailey, J. N., Jorgensen, M. J., Breidenthal, S. E., Ophoff, R. A., Comuzzie, A. G., Martin, L. J., \& Rogers, J. (2004). Genetic 
contributions to social impulsivity and aggressiveness in vervet monkeys. Biological Psychiatry, 55(6), 642-647.

Faustino, A. I., Tacão-Monteiro, A., \& Oliveira, R. F. (2017). Mechanisms of social buffering of fear in zebrafish. Scientific Reports, 7, 44329.

Federenko, I. S., Nagamine, M., Hellhammer, D. H., Wadhwa, P. D., \& Wüst, S. (2004). The heritability of hypothalamus pituitary adrenal axis responses to psychosocial stress is context dependent. The Journal of Clinical Endocrinology \& Metabolism, $89(12), 6244-6250$.

Ferguson, B., Hunter, J. E., Luty, J., Street, S. L., Woodall, A., \& Grant, K. A. (2012). Genetic load is associated with hypothalamic-pituitary-adrenal axis dysregulation in macaques. Genes, Brain and Behavior, 11(8), 949-957.

Ferguson, C. J. (2010). Genetic contributions to antisocial personality and behavior: a meta-analytic review from an evolutionary perspective. The Journal of Social Psychology, 150(2), 160-180.

Feurer, C., McGeary, J. E., Knopik, V. S., Brick, L. A., Palmer, R. H., \& Gibb, B. E. (2017). HPA axis multilocus genetic profile score moderates the impact of interpersonal stress on prospective increases in depressive symptoms for offspring of depressed mothers. Journal of Abnormal Psychology, 126(8), 1017-1028.

Finkler, H., \& Terkel, J. (2010). Cortisol levels and aggression in neutered and intact freeroaming female cats living in urban social groups. Physiology \& Behavior, 99(3), 343-347.

Fletcher, G. J., Simpson, J. A., Campbell, L., \& Overall, N. C. (2015). Pair-bonding, romantic love, and evolution: the curious case of Homo sapiens. Perspectives on Psychological Science, 10(1), 20-36.

Fleury, I., Beaulieu, P., Primeau, M., Labuda, D., Sinnett, D., \& Krajinovic, M. (2003). Characterization of the BclI polymorphism in the glucocorticoid receptor gene. Clinical Chemistry, 49(9), 1528-1531.

Flint, J. (2011). Mapping quantitative traits and strategies to find quantitative trait genes. Methods, 53(2), 163-174.

Fooden, J. (1971). Report on primates collected in western Thailand January-April, 1967. Fieldiana Zoology, 59(1), 1-62.

Fooden, J. (1980). Classification and distribution of living macaques (Macaca Lacépède, 1799). In Lindburg, D. G., (Ed.), The macaques: studies in ecology, behaviour and evolution,1-9. New York: Van Nostrand Reinhold.

Fooden, J. (1982). Taxonomy and evolution of the sinica group of macaques: 3. Species and subspecies account of Macaca assamensis. Fieldiana Zoology, 10, 1-52.

Fowler, J. H., Dawes, C. T., \& Christakis, N. A. (2009). Model of genetic variation in human social networks. Proceedings of the National Academy of Sciences of the United States of America, 106(6), 1720-1724.

Fowler, J. H., Settle, J. E., \& Christakis, N. A. (2011). Correlated genotypes in friendship networks. Proceedings of the National Academy of Sciences of the United States of America, 108(5), 1993-1997. 
Fox, J., \& Weisberg, S. (2011). An R Companion to applied regression. Thousand Oaks: Sage Publications.

Fujii, T., Hori, H., Ota, M., Hattori, K., Teraishi, T., Sasayama, D., Yamamoto, N., Higuchi, T., \& Kunugi, H. (2014). Effect of the common functional FKBP5 variant (rs1360780) on the hypothalamic-pituitary-adrenal axis and peripheral blood gene expression. Psychoneuroendocrinology, 42, 89-97.

Fuller, R. W. (1995). Serotonin receptors involved in regulation of pituitaryadrenocortical function in rats. Behavioural Brain Research, 73(1-2), 215-219.

Furlong, R. A., Ho, L., Rubinsztein, J. S., Walsh, C., Paykel, E. S., \& Rubinsztein, D. C. (1999). Analysis of the monoamine oxidase A (MAOA) gene in bipolar affective disorder by association studies, meta-analyses, and sequencing of the promoter. American Journal of Medical Genetics Part A, 88(4), 398-406.

Fürtbauer, I., Schülke, O., Heistermann, M., \& Ostner, J. (2010). Reproductive and life history parameters of wild female Macaca assamensis. International Journal of Primatology, 31(4), 501-517.

Fürtbauer, I., Heistermann, M., Schülke, O., \& Ostner, J. (2011a). Concealed fertility and extended female sexuality in a non-human primate (Macaca assamensis). PLOS ONE, 6(8), e23105.

Fürtbauer, I., Mundry, R., Heistermann, M., Schülke, O., \& Ostner, J. (2011b). You mate, I mate: macaque females synchronize sex not cycles. PLOS ONE, 6(10), 6-11.

Fürtbauer, I., Heistermann, M., Schülke, O., \& Ostner, J. (2013). Brief communication: female fecal androgens prior to the mating season reflect readiness to conceive in reproductively quiescent wild macaques. American Journal of Physical Anthropology, 151(2), 311-315.

Gallardo-Pujol, D., Andrés-Pueyo, A., \& Maydeu-Olivares, A. (2013). MAOA genotype, social exclusion and aggression: an experimental test of a gene-environment interaction. Genes, Brain and Behavior, 12(1), 140-145.

Galvão-Coelho, N. L., Silva, H. P. A., \& De Sousa, M. B. C. (2012). The influence of sex and relatedness on stress response in common marmosets (Callithrix jacchus). American Journal of Primatology, 74(9), 819-827.

Garamszegi, L. Z., Mueller, J. C., Markó, G., Szász, E., Zsebők, S., Herczeg, G., Eens, M., \& Török, J. (2014). The relationship between DRD4 polymorphisms and phenotypic correlations of behaviors in the collared flycatcher. Ecology and Evolution, 4(8), 1466-1479.

Georgiev, A. V, Klimczuk, A. C. E., Traficonte, D. M., \& Maestripieri, D. (2013). When violence pays: a cost-benefit analysis of aggressive behavior in animals and humans. Evolutionary Psychology, 11(3), 678-699.

Gero, S., Engelhaupt, D., \& Whitehead, H. (2008). Heterogeneous social associations within a sperm whale, Physeter macrocephalus, unit reflect pairwise relatedness. Behavioral Ecology and Sociobiology, 63(1), 143-151.

Geyer, S., Matelli, M., Luppino, G., \& Zilles, K. (2000). Functional neuroanatomy of the primate isocortical motor system. Anatomy and Embryology, 202(6), 443-474. 
Gibbs, R. A., Weinstock, G. M., Metzker, M. L., Muzny, D. M., Sodergren, E. J., Scherer, S., Scott, G., Steffen, D., Worley, K. C., \& Burch, P. E. (2004). Genome sequence of the brown norway rat yields insights into mammalian evolution. Nature, 428(6982), 493-521.

Gibbs, R. A., Rogers, J., Katze, M. G., Bumgarner, R., Weinstock, G. M., Mardis, E. R., Remington, K. A., Strausberg, R. L.,Venter, J. C., \& Wilson, R. K. (2007). Evolutionary and biomedical insights from the rhesus macaque genome. Science, $316,222-234$.

Godbout, J. P., \& Glaser, R. (2006). Stress-induced immune dysregulation: implications for wound healing, infectious disease and cancer. Journal of Neuroimmune Pharmacology, 1(4), 421-427.

Goeders, N. E. (2003). The impact of stress on addiction. European Neuropsychopharmacology, 13(6), 435-441.

Gogos, J. A., Morgan, M., Luine, V., Santha, M., Ogawa, S., Pfaff, D., \& Karayiorgou, M. (1998). Catechol-O-methyltransferase-deficient mice exhibit sexually dimorphic changes in catecholamine levels and behavior. Proceedings of the National Academy of Sciences of the United States of America, 95(17), 9991-9996.

Goldman-Rakic, P. S., Muly III, E. C., \& Williams, G. V. (2000). D1 receptors in prefrontal cells and circuits. Brain Research Reviews, 31(2-3), 295-301.

Goldman, D. (2014). Genomics of impulsivity: integrating genes and neuroscience. In Stoltenberg, S.F. (Ed.), Genes and the motivation to use substances, 129-139. New York: Springer.

Goldman, D., Buzas, B., \& Xu, K. (2009). Functional alleles, neuroimaging and intermediate phenotypes in the deconstruction of complex behavioral variation. In Rumsey, J. M., \& Ernst, M. (Eds.), Neuroimaging in developmental clinical neuroscience, 365-382. Cambridge: Cambridge University Press.

Goldman, D., Oroszi, G., \& Ducci, F. (2005). The genetics of addictions: uncovering the genes. Nature Reviews Genetics, 6(7), 521-532.

Goodrich, J. K., Di Rienzi, S. C., Poole, A. C., Koren, O., Walters, W. A., Caporaso, J. G., Knight, R., \& Ley, R. E. (2014). Conducting a microbiome study. Cell, 158(2), 250-262.

Goodyer, I. M., Bacon, A., Ban, M., Croudace, T., \& Herbert, J. (2009). Serotonin transporter genotype, morning cortisol and subsequent depression in adolescents. The British Journal of Psychiatry, 195(1), 39-45.

Gosso, M. F., De Geus, E. J. C., Polderman, T. J. C., Boomsma, D. I., Heutink, P., \& Posthuma, D. (2008). Catechol O-methyl transferase and dopamine D2 receptor gene polymorphisms: evidence of positive heterosis and gene-gene interaction on working memory functioning. European Journal of Human Genetics, 16(9), 10751082.

Gotlib, I. H., Joormann, J., Minor, K. L., \& Hallmayer, J. (2018). HPA axis reactivity: a mechanism underlying the associations among 5-HTTLPR, stress, and depression. Biological Psychiatry, 63(9), 847-851.

Goymann, W. (2005). Noninvasive monitoring of hormones in bird droppings: 
physiological validation, sampling, extraction, sex differences, and the influence of diet on hormone metabolite levels. Annals of the New York Academy of Sciences, $1046,35-53$.

Goymann, W., Wingfield, J. C., Planck, M., \& Mcewen, F. (2004). Allostatic load, social status and stress hormones: the costs of social status matter. Animal Behaviour, 67(3), 591-602.

Grady, D. L., Thanos, P. K., Corrada, M. M., Barnett, J. C., Ciobanu, V., Shustarovich, D., Napoli, A., Moyzis, A. G., Grandy, D., Rubinstein, M., Wang, G.-J., H.Kawas, C., Chen, C., Dong, Q., Wang, E., Volkow, N. D, \& Moyzis, R. K. (2013). DRD4 genotype predicts longevity in mouse and human. The Journal of Neuroscience, 33(1), 286-291.

Grassman, L. I., Tewes, M. E., \& Silvy, N. J. (2005). Ranging, habitat use and activity patterns of binturong Arctictis binturong and yellow-throated marten Martes flavigula in north-central Thailand. Wildlife Biology, 11(1), 49-57.

Green, J. R., \& Korenberg, M. J. (2006). Nonlinear system identification provides insight into protein folding. IEEE Canadian Conference on Electrical and Computer Engineering Ottawa, Ontario 2006

Green, J. R., Korenberg, M. J., \& Aboul-Magd, M. O. (2009). PCI-SS: MISO dynamic nonlinear protein secondary structure prediction. BMC Bioinformatics, 10(1), 222.

Grossman, M. H., Emanuel, B. S., \& Budarf, M. L. (1992). Chromosomal mapping of the human catechol-O-methyltransferase gene to 22q11. $1 \rightarrow$ q11. 2. Genomics, 12(4), $822-825$.

Groves, J. O. (2007). Is it time to reassess the BDNF hypothesis of depression? Molecular Psychiatry, 12, 1079-1088.

Gualtieri, C. T., \& Schroeder, S. R. (1990). Pharmacotherapy for self-injurious behavior: preliminary tests of the D1 hypothesis. Progress in Neuro-Psychopharmacology and Biological Psychiatry, 14, 81-107.

Guhathakurta, S., Sinha, S., Ghosh, S., Chatterjee, A., Ahmed, S., Gangopadhyay, P. K., \& Usha, R. (2008). Population-based association study and contrasting linkage disequilibrium pattern reveal genetic association of SLC6A4 with autism in the indian population from West Bengal. Brain Research, 1240, 12-21.

Gunnar, M. R., Gonzalez, C. A., \& Levine, S. (1980). The role of peers in modifying behavioral distress and pituitary-adrenal response to a novel environment in year-old rhesus monkeys. Physiology \& Behavior, 25(5), 795-798.

Gunnar, M. R., \& Hostinar, C. E. (2015). The social buffering of the hypothalamicpituitary-adrenocortical axis in humans: developmental and experiential determinants. Social Neuroscience, 10(5), 479-488.

Gutleb, D. R., Ostner, J., Schülke, O., Wajjwalku, W., Sukmak, M., Roos, C., \& Noll, A. (2018a). Non-invasive genotyping with a massively parallel sequencing panel for the detection of SNPs in HPA-axis genes. Scientific Reports, 8, 15944.

Gutleb, D. R., Roos, C., Noll, A., Ostner, J., \& Schülke, O. (2018b). COMT Val ${ }^{158}$ Met moderates the link between rank and aggression in a non-human primate. Genes, Brain and Behavior, 17(4), e12443. 
Habig, B., \& Archie, E. A. (2015). Social status, immune response and parasitism in males: a meta-analysis. Philosophical Transactions of the Royal Society of London B: Biological Sciences, 370(1669), 20140109.

Häggström, M., \& Richfield, D. (2014). Diagram of the pathways of human steroidogenesis. WikiJournal of Medicine, 1(1), 1-5.

Halász, J., Liposits, Z., Kruk, M. R., \& Haller, J. (2002). Neural background of glucocorticoid dysfunction-induced abnormal aggression in rats: involvement of fear-and stress-related structures. European Journal of Neuroscience, 15(3), 561569.

Hamilton, W. D. (1964). The genetical evolution of social behaviour I-II. Journal of Theoretical Biology, 7(1), 1-52.

Han, D. H., Park, D. B., Na, C., Kee, B. S., \& Lee, Y. S. (2004). Association of aggressive behavior in Korean male schizophrenic patients with polymorphisms in the serotonin transporter promoter and catecholamine-O-methyltransferase genes. Psychiatry Research, 129(1), 29-37.

Han, D. H., Kee, B. S., Min, K. J., Lee, Y. S., Na, C., Park, D. B., \& Lyoo, I. K. (2006). Effects of catechol-O-methyltransferase Val158Met polymorphism on the cognitive stability and aggression in the first-onset schizophrenic patients. Neuroreport, 17(1), 95-99.

Harcourt, A. H. (1992). Coalitions and alliances: are primates more complex than nonprimates. In Harcourt, A. H., \& de Waal, F. B. M. (Eds.), Coalitions and alliances in humans and other animals, 445-471. Oxford: Oxford University Press.

Hauer, D., Weis, F., Papassotiropoulos, A., Schmoeckel, M., Beiras-Fernandez, A., Lieke, J., Kaufmann, I., Kirchhoff, F., Vogeser, M., Roozendaal, B., Briegel, J., de Quervain, D., \& Schelling, G. (2011). Relationship of a common polymorphism of the glucocorticoid receptor gene to traumatic memories and posttraumatic stress disorder in patients after intensive care therapy. Critical Care Medicine, 39(4), 643650.

Haunhorst, C. B. (2017). Evolutionary origin of the human pair-bond - the adaptive significance of male-female relationships in wild Assamese macaques (Macaca assamensis), Thailand. Dissertation University of Goettingen.

Haunhorst, C. B., Schülke, O., \& Ostner, J. (2016). Opposite-sex social bonding in wild Assamese macaques. American Journal of Primatology, 78(8), 872-882.

Haunhorst, C. B., Heesen, M., Ostner, J., \& Schülke, O. (2017). Social bonds with males lower the costs of competition for wild female Assamese macaques. Animal Behaviour, 125, 51-60.

Hawkley, L. C., Cole, S. W., Capitanio, J. P., Norman, G. J., \& Cacioppo, J. T. (2012). Effects of social isolation on glucocorticoid regulation in social mammals. Hormones and Behavior, 62(3), 314-323.

Hawkley, L. C., \& Capitanio, J. P. (2015). Perceived social isolation, evolutionary fitness and health outcomes: a lifespan approach. Philosophical Transactions of the Royal Society of London B: Biological Sciences, 370(1669), 20140114.

Heesen, M., Macdonald, S., Ostner, J., \& Schülke, O. (2015). Ecological and social 
determinants of group cohesiveness and within-group spatial position in wild Assamese macaques. Ethology, 121(3), 270-283.

Heesen, M., Rogahn, S., Macdonald, S., Ostner, J., \& Schülke, O. (2014). Predictors of food-related aggression in wild Assamese macaques and the role of conflict avoidance. Behavioral Ecology and Sociobiology, 68(11), 1829-1841.

Heesen, M., Rogahn, S., Ostner, J., \& Schülke, O. (2013). Food abundance affects energy intake and reproduction in frugivorous female Assamese macaques. Behavioral Ecology and Sociobiology, 67(7), 1053-1066.

Heinrichs, M., Baumgartner, T., Kirschbaum, C., \& Ehlert, U. (2003). Social support and oxytocin interact to suppress cortisol and subjective responses to psychosocial stress. Biological Psychiatry, 54(12), 1389-1398.

Heistermann, M. (2010). Non-invasive monitoring of endocrine status in laboratory primates: methods, guidelines and applications. Advances in Science and Research, $5,1-9$.

Hennessy, M. B., Hornschuh, G., Kaiser, S., \& Sachser, N. (2006). Cortisol responses and social buffering: a study throughout the life span. Hormones and Behavior, 49(3), 383-390.

Hennessy, M. B., Kaiser, S., \& Sachser, N. (2009). Social buffering of the stress response: diversity, mechanisms, and functions. Frontiers in Neuroendocrinology, $30(4), 470-482$.

Herman, J. P., McKlveen, J. M., Ghosal, S., Kopp, B., Wulsin, A., Makinson, R., Scheimann, J., \& Myers, B. (2016). Regulation of the hypothalamic-pituitaryadrenocortical stress response. Comprehensive Physiology, 6(2), 603-621.

Hess, G. T., Tycko, J., Yao, D., \& Bassik, M. C. (2017). Methods and applications of CRISPR-mediated base editing in eukaryotic genomes. Molecular Cell, 68(1), 2643.

Higashino, A., Sakate, R., Kameoka, Y., Takahashi, I., Hirata, M., Tanuma, R., Masui, T., Yasutomi, Y., \& Osada, N. (2012). Whole-genome sequencing and analysis of the Malaysian cynomolgus macaque (Macaca fascicularis) genome. Genome Biology, 13(7), R58.

Higham, J. P., Barr, C. S., Hoffman, C. L., Mandalaywala, T. M., Parker, K. J., \& Maestripieri, D. (2011). Mu-opioid receptor (OPRM1) variation, oxytocin levels and maternal attachment in free-ranging rhesus macaques Macaca mulatta. Behavioral Neuroscience, 125(2), 131-136.

Hill, L. D., Lorenzetti, M. S., Lyle, S. M., Fins, A. I., Tartar, A., \& Tartar, J. L. (2018). Catechol-O-methyltransferase Val158Met polymorphism associates with affect and cortisol levels in women. Brain and Behavior, 8(2), e00883.

Hinde, R. A., \& Atkinson, S. (1970). Assessing the roles of social partners in maintaining mutual proximity, as exemplified by mother-infant relations in rhesus monkeys. Animal Behaviour, 18, 169-176.

Hirsch, D., \& Zukowska, Z. (2012). NPY and stress 30 years later: the peripheral view. Cellular and Molecular Neurobiology, 32(5), 645-659. 
Holekamp, K. E., Cooper, S. M., Katona, C. I., Berry, N. A., Frank, L. G., \& Smale, L. (1997). Patterns of association among female spotted hyenas (Crocuta crocuta). Journal of Mammalogy, 78(1), 55-64.

Holliday, K. L., Nicholl, B. I., Macfarlane, G. J., Thomson, W., Davies, K. A., \& McBeth, J. (2010). Genetic variation in the hypothalamic-pituitary-adrenal stress axis influences susceptibility to musculoskeletal pain: results from the EPIFUND study. Annals of the Rheumatic Diseases, 69(3), 556-560.

Holmes, A., Murphy, D. L., \& Crawley, J. N. (2002). Reduced aggression in mice lacking the serotonin transporter. Psychopharmacology, 161(2), 160-167.

Holt-Lunstad, J., Smith, T. B., \& Layton, J. B. (2010). Social relationships and mortality risk: a meta-analytic review. PLoS Medicine, 7(7), e1000316.

Honess, P. E., \& Marin, C. M. (2006). Behavioural and physiological aspects of stress and aggression in nonhuman primates. Neuroscience and Biobehavioral Reviews, 30(3), 390-412.

Hong, J., Shu-Leong, H., Tao, X., \& Lap-Ping, Y. (1998). Distribution of catechol-O-methyltransferase expression in human central nervous system. Neuroreport, 9(12), 2861-2864.

Hosang, G. M., Fisher, H. L., Cohen-Woods, S., McGuffin, P., \& Farmer, A. E. (2017). Stressful life events and catechol-O-methyl-transferase (COMT) gene in bipolar disorder. Depression and Anxiety, 34(5), 419-426.

Hosang, G. M., Shiles, C., Tansey, K. E., McGuffin, P., \& Uher, R. (2014). Interaction between stress and the BDNF Val66Met polymorphism in depression: a systematic review and meta-analysis. BMC Medicine, 12(1), 7.

Hostinar, C. E., Sullivan, R. M., \& Gunnar, M. R. (2014). Psychobiological mechanisms underlying the social buffering of the hypothalamic-pituitary-adrenocortical axis: a review of animal models and human studies across development. Psychological Bulletin, 140(1), 256-282.

House, J. S., Landis, K. R., \& Umberson, D. (1988). Social relationships and health. Science, 241, 540-545.

House, J. S., Robbins, C., \& Metzner, H. L. (1982). The association of social relationships and activities with mortality: prospective evidence from the Tecumseh Community Health Study. American Journal of Epidemiology, 116(1), 123-140.

Huhman, K. L., Moore, T. O., Ferris, C. F., Mougey, E. H., \& Meyerhoff, J. L. (1991). Acute and repeated exposure to social conflict in male golden hamsters: increases in plasma POMC-peptides and cortisol and decreases in plasma testosterone. Hormones and Behavior, 25(2), 206-216.

Huizenga, N. A. T. M., Koper, J. W., de Lange, P., Pols, H. A. P., Stolk, R. P., Burger, H., Grobbee, D. E., Brinkmann, A. O., de Jong, F. H., \& Lamberts, S. W. J. (1998). A polymorphism in the glucocorticoid receptor gene may be associated with an increased sensitivity to glucocorticoids in vivo 1. The Journal of Clinical Endocrinology \& Metabolism, 83(1), 144-151.

Hutchins, M., \& Barash, D. P. (1976). Grooming in primates: implications for its utilitarian function. Primates, 17(2), 145-150. 
Hygen, B. W., Belsky, J., Stenseng, F., Lydersen, S., Guzey, I. C., \& Wichstrøm, L. (2015). Child exposure to serious life events, COMT, and aggression: testing differential susceptibility theory. Developmental Psychology, 51(8), 1098-1104.

Iervolino, A. C., Pike, A., Manke, B., Reiss, D., Hetherington, E. M., \& Plomin, R. (2002). Genetic and environmental influences in adolescent peer socialization: evidence from two genetically sensitive designs. Child Development, 73(1), 162174.

Inoue-Murayama, M. (2009). Genetic polymorphism as a background of animal behavior. Animal Science Journal, 80(2), 113-120.

Inoue-Murayama, M., Inoue, E., Watanabe, K., Takenaka, A., \& Murayama, Y. (2010). Behavior-related candidate genes in Japanese macaques. In Nakagawa, N., Nakamichi, M., \& Sugiura, H. (Eds.), The Japanese macaques, 293-301. Tokyo: Springer.

Inoue-Murayama, M., Yokoyama, C., Yamanashi, Y., \& Weiss, A. (2018). Common marmoset (Callithrix jacchus) personality, subjective well-being, hair cortisol level and AVPR1a, OPRM1, and DAT genotypes. Scientific Reports, 8, 10255.

Ising, M., \& Holsboer, F. (2006). Genetics of stress response and stress-related disorders. Dialogues in Clinical Neuroscience, 8(4), 433-444.

Iurescia, S., Seripa, D., \& Rinaldi, M. (2016). Looking beyond the 5-HTTLPR polymorphism: genetic and epigenetic layers of regulation affecting the serotonin transporter gene expression. Molecular Neurobiology, 54(10), 8386-8403.

Jabbi, M., Korf, J., Kema, I. P., Hartman, C., van der Pompe, G., Minderaa, R. B., Ormel, J., \& den Boer, J. A. (2007). Convergent genetic modulation of the endocrine stress response involves polymorphic variations of 5-HTT, COMT and MAOA. Molecular Psychiatry, 12(5), 483-490.

Jansen, F., Heiming, R. S., Lewejohann, L., Touma, C., Palme, R., Schmitt, A., Lesch, K. P. \& Sachser, N. (2010). Modulation of behavioural profile and stress response by 5HTT genotype and social experience in adulthood. Behavioural Brain Research, 207(1), 21-29.

Jardine, D., Emond, M., Meert, K. L., Harrison, R., Carcillo, J. A., Anand, K. J. S., Berger, J., Newth, C. J. L., Willson, D. F., Nicholson, C., Dean, J. M., \& Zimmerman, J. J. (2014). A single nucleotide polymorphism in the corticotropin receptor gene is associated with a blunted cortisol response during pediatric critical illness. Pediatric Critical Care Medicine, 15(8), 1-8.

Josephs, N., Bonnell, T., Dostie, M., Barrett, L., \& Henzi, S. P. (2016). Working the crowd: sociable vervets benefit by reducing exposure to risk. Behavioral Ecology, 27(4), 988-994.

Käenmäki, M., Tammimäki, A., Myöhänen, T., Pakarinen, K., Amberg, C., Karayiorgou, M., Gogos, Joseph A., \& Männistö, P. T. (2010). Quantitative role of COMT in dopamine clearance in the prefrontal cortex of freely moving mice. Journal of Neurochemistry, 114(6), 1745-1755.

Kaewpanus, K., Aggimarangsee, N., Sitasuwan, N., \& Wangpakapattanawong, P. (2015). Diet and feeding behavior of Assamese macaques (Macaca assamensis) at Tham Pla 
temple, Chiang Rai province, northern Thailand. Journal of Wildlife in Thailand, 22(1), 23-35.

Kaidanovich-Beilin, O., Lipina, T., Vukobradovic, I., Roder, J., \& Woodgett, J. R. (2011). Assessment of social interaction behaviors. Journal of Visualized Experiments, 48, e2473.

Kalbitz, J., Ostner, J., \& Schülke, O. (2016). Strong, equitable and long-term social bonds in the dispersing sex in Assamese macaques. Animal Behaviour, 113, 13-22.

Kalbitz, J., Schülke, O., \& Ostner, J. (2017). Triadic male-infant-male interaction serves in bond maintenance in male Assamese macaques. PLOS ONE, 12(10), e0183981.

Kalbitzer, U., Bergstrom, M. L., Carnegie, S. D., Wikberg, E. C., Kawamura, S., Campos, F. A., Jack, K. M., \& Fedigan, L. M. (2017). Female sociality and sexual conflict shape offspring survival in a neotropical primate. Proceedings of the National Academy of Sciences of the United States of America, 114(8), 1892-1897.

Kalbitzer, U., Roos, C., Kopp, G. H., Butynski, T. M., Knauf, S., Zinner, D., \& Fischer, J. (2016). Insights into the genetic foundation of aggression in Papio and the evolution of two length-polymorphisms in the promoter regions of serotonin-related genes (5HTTLPR and MAOALPR) in Papionini. BMC Evolutionary Biology, 16(1), 121.

Kalin, N. H., Shelton, S. E., \& Davidson, R. J. (2007). Role of the primate orbitofrontal cortex in mediating anxious temperament. Biological Psychiatry, 62(10), 11341139.

Kanehisa, M., Furumichi, M., Tanabe, M., Sato, Y., \& Morishima, K. (2017). KEGG: new perspectives on genomes, pathways, diseases and drugs. Nucleic Acids Research, 45(1), 353-361.

Kanehisa, M., Sato, Y., Kawashima, M., Furumichi, M., \& Tanabe, M. (2016). KEGG as a reference resource for gene and protein annotation. Nucleic Acids Research, 44(1), $457-462$.

Karere, G. M., Sullivan, E., Kinnally, E. L., Capitanio, J. P., \& Lyons, L. A. (2012). Enhancing genotyping of MAOA-LPR and 5-HTT-LPR in rhesus macaques (Macaca mulatta). Journal of Medical Primatology, 41(6), 407-411.

Karoum, F., Chrapusta, S. J., \& Egan, M. F. (1994). 3-Methoxytyramine is the major metabolite of released dopamine in the rat frontal cortex: reassessment of the effects of antipsychotics on the dynamics of dopamine delease and metabolism in the frontal cortex, nucleus accumbens, and striatum by a simple. Journal of Neurochemistry, 63(3), 972-979.

Kathiresan, S., Melander, O., Anevski, D., Guiducci, C., Burtt, N. P., Roos, C., Hirschhorn, J. N., Berglund, G., Hedblad, B., \& Groop, L. (2008). Polymorphisms associated with cholesterol and risk of cardiovascular events. New England Journal of Medicine, 358(12), 1240-1249.

Keller, C., Roos, C., Groeneveld, L. F., Fischer, J., \& Zinner, D. (2010). Introgressive hybridization in southern African baboons shapes patterns of mtDNA variation. American Journal of Physical Anthropology, 142(1), 125-136.

Kent, W. J. (2002). BLAT - the BLAST-like alignment tool. Genome Research, 12(4), 656-664. 
Kent, W. J., Sugnet, C. W., Furey, T. S., Roskin, K. M., Pringle, T. H., Zahler, A. M., \& Haussler, D. (2002). The human genome browser at UCSC. Genome Research, 12(6), 996-1006.

Kia-Keating, B. M., Glatt, S. J., \& Tsuang, M. T. (2007). Meta-analyses suggest association between COMT, but not HTR1B, alleles, and suicidal behavior. American Journal of Medical Genetics Part B: Neuropsychiatric Genetics, 144(8), 1048-1053.

Kikusui, T., Winslow, J. T., \& Mori, Y. (2006). Social buffering: relief from stress and anxiety. Philosophical Transactions of the Royal Society B: Biological Sciences, $361,2215-2228$.

King, B. R., \& Nicholson, R. C. (2007). Advances in understanding corticotrophinreleasing hormone gene expression. Frontiers in Bioscience, 12(2), 581-590.

Kircher, M., Sawyer, S., \& Meyer, M. (2012). Double indexing overcomes inaccuracies in multiplex sequencing on the Illumina platform. Nucleic Acids Research, 40(1), e3.

Kirschbaum, C., Klauer, T., Filipp, S.-H., \& Hellhammer, D. H. (1995). Sex-specific effects of social support on cortisol and subjective responses to acute psychological stress. Psychosomatic Medicine, 57(1), 23-31.

Kirschbaum, C., Pirke, K.-M., \& Hellhammer, D. H. (1993). The 'Trier Social Stress Test'-a tool for investigating psychobiological stress responses in a laboratory setting. Neuropsychobiology, 28(1-2), 76-81.

Kirschbaum, C., Wüst, S., Faig, H. G., \& Hellhammer, D. H. (1992). Heritability of cortisol responses to human corticotropin-releasing hormone, ergometry, and psychological stress in humans. The Journal of Clinical Endocrinology \& Metabolism, 75(6), 1526-1530.

Kiyokawa, Y., \& Hennessy, M. B. (2018). Comparative studies of social buffering: a consideration of approaches, terminology, and pitfalls. Neuroscience \& Biobehavioral Reviews, 86, 131-141.

Koenig, A., Borries, C., Suarez, S., Kreetiyutanont, K., \& Prabnasuk, J. (2004a). Socioecology of Phayre's leaf monkeys (Trachypithecus phayrei) at Phu Khieo Wildlife Sanctuary. Journal of Wildlife in Thailand, 12, 150-163.

Koenig, A., Larney, E., Lu, A., \& Borries, C. (2004b). Agonistic behavior and dominance relationships in female Phayre's leaf monkeys-preliminary results. American Journal of Primatology, 64(3), 351-357.

Kohn, J. N., Snyder-Mackler, N., Barreiro, L. B., Johnson, Z. P., Tung, J., \& Wilson, M. E. (2016). Dominance rank causally affects personality and glucocorticoid regulation in female rhesus macaques. Psychoneuroendocrinology, 74, 179-188.

Koirala, S., Chalise, M. K., Katuwal, H. B., Gaire, R., Pandey, B., \& Ogawa, H. (2017). Diet and activity of Macaca assamensis in wild and semi-provisioned groups in Shivapuri Nagarjun National Park, Nepal. Folia Primatologica, 88(2), 57-74.

König, B., Lindholm, A. K., Lopes, P. C., Dobay, A., Steinert, S., \& Buschmann, F. J.-U. (2015). A system for automatic recording of social behavior in a free-living wild house mouse population. Animal Biotelemetry, 3(1), 39. 
Koolhaas, J. M., de Boer, S. F., Buwalda, B., \& Meerlo, P. (2017). Social stress models in rodents: towards enhanced validity. Neurobiology of Stress, 6, 104-112.

Kopp, G. H., Fischer, J., Patzelt, A., Roos, C., \& Zinner, D. (2015). Population genetic insights into the social organization of Guinea baboons (Papio papio): evidence for female-biased dispersal. American Journal of Primatology, 77(8), 878-889.

Kormos, V., \& Gaszner, B. (2013). Role of neuropeptides in anxiety, stress, and depression: from animals to humans. Neuropeptides, 47(6), 401-419.

Krawczak, M., Trefilov, A., Berard, J., Bercovitch, F., Kessler, M., Sauermann, U., Croucher, P., Nürnberg, P., Widdig, A., \& Widdig, A. (2005). Male reproductive timing in rhesus macaques is influenced by the 5HTTLPR promoter polymorphism of the serotonin transporter gene 1, Biology of Reproduction, 72(5), 1109-1113.

Kruk, M. R., Halász, J., Meelis, W., \& Haller, J. J. (2004). Fast positive feedback between the adrenocortical stress response and a brain mechanism involved in aggressive behavior. Behavioral Neuroscience, 118(5), 1062-1070.

Kruuk, L. E. B. (2004). Estimating genetic parameters in natural populations using the "animal model". Philosophical Transactions of the Royal Society B: Biological Sciences, 359(1446), 873-890.

Kudielka, B. M., Buske-Kirschbaum, A., Hellhammer, D. H., \& Kirschbaum, C. (2004). HPA axis responses to laboratory psychosocial stress in healthy elderly adults, younger adults, and children: impact of age and gender. Psychoneuroendocrinology, 29(1), 83-98.

Kudielka, B. M., \& Kirschbaum, C. (2005). Sex differences in HPA axis responses to stress: a review. Biological Psychology, 69(1), 113-132.

Kumar, S., \& Hedges, S. B. (1998). A molecular timescale for vertebrate evolution. Nature, 392(6679), 917-920.

Kuperman, S., Kramer, J., \& Loney, J. (1988). Enzyme activity and behavior in hyperactive children grown up. Biological Psychiatry, 24(4), 375-383.

Kuznetsova, A., Brockhoff, P. B., \& Christensen, R. H. B. (2016). lmerTest package: tests in linear mixed effects models. Journal of Statistical Software, 82(13).

Kwon, J. M., \& Goate, A. M. (2000). The candidate gene approach. Alcohol Research and Health, 24(3), 164-168.

Lachman, H. M., Nolan, K. A., Mohr, P., Saito, T., \& Volavka, J. (1998). Association between catechol O-methyltransferase genotype and violence in schizophrenia and schizoaffective disorder. American Journal of Psychiatry, 155(6), 835-837.

Larhammar, D., \& Salaneck, E. (2004). Molecular evolution of NPY receptor subtypes. Neuropeptides, 38(4), 141-151.

Larson, C. L., Taubitz, L. E., \& Robinson, J. S. (2010). MAOA T941G polymorphism and the time course of emotional recovery following unpleasant pictures. Psychophysiology, 47(5), 857-862.

Laucht, M., Blomeyer, D., Buchmann, A. F., Treutlein, J., Schmidt, M. H., Esser, G., Jennen-Steinmetz, C., Rietschel, M., Zimmermann, U. S. \& Banaschewski, T. 
(2012). Catechol-O-methyltransferase Val158Met genotype, parenting practices and adolescent alcohol use: testing the differential susceptibility hypothesis. Journal of Child Psychology and Psychiatry, 53(4), 351-359.

Laudet, V., Hänni, C., Coll, J., Catzeflis, F., \& Stéhelin, D. (1992). Evolution of the nuclear receptor gene superfamily. The EMBO Journal, 11(3), 1003-1013.

Lazzaretti, M., Fabbro, D., Sala, M., Del Toso, K., De Vidovich, G., Marraffini, E., Morandotti, N., Gambini, F., Barale, F., \& Balestrieri, M. (2013). Association between low-activity allele of cathecolamine-O-methyl-transferase (COMT) and borderline personality disorder in an Italian population. Behavioral Medicine, 39(2), $25-28$.

Lea, A. J., Blumstein, D. T., Wey, T. W., \& Martin, J. G. A. (2010). Heritable victimization and the benefits of agonistic relationships. Proceedings of the National Academy of Sciences of the United States of America, 107(50), 21587-21592.

Lee, R. M., \& Robbins, S. B. (1998). The relationship between social connectedness and anxiety, self-esteem, and social identity. Journal of Counseling Psychology, 45(3), $338-345$.

Lee, R. M., \& Robbins, S. B. (2000). Understanding social connectedness in college women and men. Journal of Counseling \& Development, 78(4), 484-491.

Lehmann, J., Majolo, B., \& McFarland, R. (2016). The effects of social network position on the survival of wild Barbary macaques, Macaca sylvanus. Behavioral Ecology, 27(1), 20-28.

Lesch, K. P., Bengel, D., Heils, a, Sabol, S. Z., Greenberg, B. D., Petri, S., Benjamin, J., Müller, C. R., Hamer, D. H. \& Murphy, D. L. (1996). Association of anxiety-related traits with a polymorphism in the serotonin transporter gene regulatory region. Science, 274(5292), 1527-1531.

Lesch, K. P., \& Merschdorf, U. (2000). Impulsivity, aggression, and serotonin: a molecular psychobiological perspective. Behavioral Sciences \& the Law, 18(5), 581-604.

Leszczyńska-Rodziewicz, A., Szczepankiewicz, A., Dmitrzak-Węglarz, M., Skibińska, M., \& Hauser, J. (2012). Association between functional polymorphism of the AVPR1b gene and polymorphism rs1293651 of the CRHR1 gene and bipolar disorder with psychotic features. Journal of Affective Disorders, 138(3), 490-493.

Levine, S., Johnson, D. F., \& Gonzalez, C. A. (1985). Behavioral and hormonal responses to separation in infant rhesus monkeys and mothers. Behavioral Neuroscience, 99(3), 399-410.

Li, H. (2013). Aligning sequence reads, clone sequences and assembly contigs with BWA-MEM. ArXiv Preprint ArXiv:1303.3997.

Li, H., Handsaker, B., Wysoker, A., Fennell, T., Ruan, J., Homer, N., Marth, G., Abecasis, G., \& Durbin, R. (2009). The sequence alignment/map format and SAMtools. Bioinformatics, 25(16), 2078-2079.

Li, J., Han, K., Xing, J., Kim, H.-S., Rogers, J., Ryder, O. A., Disotell, T., Yue, B., \& Batzer, M. A. (2009). Phylogeny of the macaques (Cercopithecidae: Macaca) based on Alu elements. Gene, 448(2), 242-249. 
Lian, Y., Xiao, J., Wang, Q., Ning, L., Guan, S., Ge, H., Li, F., \& Liu, J. (2014). The relationship between glucocorticoid receptor polymorphisms, stressful life events, social support, and post-traumatic stress disorder. BMC Psychiatry, 14(1), 232.

Lindell, S. G., Schwandt, M. L., Sun, H., Sparenborg, J. D., Björk, K., Kasckow, J. W., Sommer, W. H., Goldman, D., Higley, J. D. \& Suomi, S. J. (2010). Functional NPY variation as a factor in stress resilience and alcohol consumption in rhesus macaques. Archives of General Psychiatry, 67(4), 423-431.

Lindzey, G., \& Thiessen, D. D. (1970). Contributions to behavior-genetic analysis: The mouse as a prototype. New York: Appleton-Century-Crofts.

Linkowski, P., van Onderbergen, A., Kerkhofs, M., Bosson, D., Mendlewicz, J., \& van Cauter, E. (1993). Twin study of the 24-h cortisol profile: evidence for genetic control of the human circadian clock. American Journal of PhysiologyEndocrinology and Metabolism, 264(2), 173-181.

Liu, H. L., \& Wang, W. C. (2003). Protein engineering to improve the thermostability of glucoamylase from Aspergillus awamori based on molecular dynamics simulations. Protein Engineering, 16(1), 19-25.

Llewellyn, C. H., Trzaskowski, M., van Jaarsveld, C. H. M., Plomin, R., \& Wardle, J. (2014). Satiety mechanisms in genetic risk of obesity. JAMA Pediatrics, 168(4), 338-344.

Loggia, M. L., Jensen, K., Gollub, R. L., Wasan, A. D., Edwards, R. R., \& Kong, J. (2011). The catechol-O-methyltransferase (COMT) val158met polymorphism affects brain responses to repeated painful stimuli. PLOS ONE, 6(11), 1-8.

Lotta, T., Vidgren, J., Tilgmann, C., Ulmanen, I., Melen, K., Julkunen, I., \& Taskinen, J. (1995). Kinetics of human soluble and membrane-bound catechol Omethyltransferase: a revised mechanism and description of the thermolabile variant of the enzyme. Biochemistry, 34(13), 4202-4210.

Lukas, D., \& Huchard, E. (2014). The evolution of infanticide by males in mammalian societies. Science, 346(6211), 841-844.

Lutgendorf, S. K., Anderson, B., Sorosky, J. I., Buller, R. E., \& Lubaroff, D. M. (2000). Interleukin-6 and use of social support in gynecologic cancer patients. International Journal of Behavioral Medicine, 7(2), 127-142.

'macaca'. (2000). The American Heritage Dictionary of the English Language. Boston: Houghton Mifflin Company.

Macdonald, S., Schülke, O., \& Ostner, J. (2013). The absence of grooming for rankrelated benefits in female Assamese macaques (Macaca assamensis). International Journal of Primatology, 34(3), 571-584.

Machius, M., Declerck, N., Huber, R., \& Wiegand, G. (2003). Kinetic stabilization of Bacillus licheniformis $\alpha$-amylase through introduction of hydrophobic residues at the surface. Journal of Biological Chemistry, 278(13), 11546-11553.

Madlon-Kay, S., Montague, M. J., Brent, L. J. N., Ellis, S., Zhong, B., Snyder-Mackler, N., Horvath, J. E., Skene, J. H. P. \& Platt, M. L. (2018). Weak effects of common genetic variation in oxytocin and vasopressin receptor genes on rhesus macaque social behavior. American Journal of Primatology, 80(10), e22873. 
Mahon, P., Zandi, P., \& Potash, J. (2013). Genetic association of FKBP5 and CRHR1 with cortisol response to acute psychosocial stress in healthy adults. Psychopharmacology, 227(2), 231-241.

Majolo, B., Lehmann, J., de Bortoli Vizioli, A., \& Schino, G. (2012). Fitness-related benefits of dominance in primates. American Journal of Physical Anthropology, 147(4), 652-660.

Männistö, P. T., \& Kaakkola, S. (1999). Catechol-O-methyltransferase (COMT): biochemistry, molecular biology, pharmacology, and clinical efficacy of the new selective COMT inhibitors. Pharmacological Reviews, 51(4), 593-628.

Manolio, T. A., Collins, F. S., Cox, N. J., Goldstein, D. B., Hindorff, L. A., Hunter, D. J., McCarthy, M. I., Ramos, E. M., Cardon, L. R., \& Chakravarti, A. (2009). Finding the missing heritability of complex diseases. Nature, 461(7265), 747-753.

Manuck, S. B., Flory, J. D., Ferrell, R. E., Mann, J. J., \& Muldoon, M. F. (2000). A regulatory polymorphism of the monoamine oxidase-A gene may be associated with variability in aggression, impulsivity, and central nervous system serotonergic responsivity. Psychiatry Research, 95(1), 9-23.

Manzoni, C., Kia, D. A., Vandrovcova, J., Hardy, J., Wood, N. W., Lewis, P. A., \& Ferrari, R. (2018). Genome, transcriptome and proteome: the rise of omics data and their integration in biomedical sciences. Briefings in Bioinformatics, 19(2), 286-302.

Maricic, T., Whitten, M., \& Pääbo, S. (2010). Multiplexed DNA sequence capture of mitochondrial genomes using PCR products. PLOS ONE, 5(11), e14004.

Martin, P., \& Bateson, P. (1993). Measuring behaviour: an introductory guide. Cambridge: Cambridge University Press.

Massen, J. J. M., \& Koski, S. E. (2014). Chimps of a feather sit together: chimpanzee friendships are based on homophily in personality. Evolution and Human Behavior, 35(1), 1-8.

Mata, R., Hau, R., Papassotiropoulos, A., \& Hertwig, R. (2012). DAT1 polymorphism is associated with risk taking in the balloon analogue risk task (BART). PLOS ONE, 7(6), e39135.

Matsumoto, M., Weickert, C. S., Akil, M., Lipska, B. K., Hyde, T. M., Herman, M. M., Kleinman, J. E., \& Weinberger, D. R. (2003). Catechol O-methyltransferase mRNA expression in human and rat brain: evidence for a role in cortical neuronal function. Neuroscience, 116(1), 127-137.

Maxson, S. C., Roubertoux, P. L., Guillot, P., \& Goldman, D. (2001). The genetics of aggression. In Bach, M. (Ed.), Prevention and control of aggression and the impact on its victims, 71-81. Boston: Springer.

Mazzocchi, F. (2015). Could big data be the end of theory in science?: A few remarks on the epistemology of data-driven science. EMBO Reports, 16(10), 1250-1255.

McCowan, B., Beisner, B., Bliss-Moreau, E., Vandeleest, J., Jin, J., Hannibal, D., \& Hsieh, F. (2016). Connections matter: social networks and lifespan health in primate translational models. Frontiers in Psychology, 22(7), 433.

McDougle, C. J., Holmes, J. P., Carlson, D. C., Pelton, G. H., Cohen, D. J., \& Price, L. H. 
(1998). A double-blind, placebo-controlled study of risperidone in adults with autistic disorder and other pervasive developmental disorders. Archives of General Psychiatry, 55(7), 633-641.

McFarland, R., Fuller, A., Hetem, R. S., Mitchell, D., Maloney, S. K., Henzi, S. P., \& Barrett, L. (2015). Social integration confers thermal benefits in a gregarious primate. Journal of Animal Ecology, 84(3), 871-878.

McFarland, R., Murphy, D., Lusseau, D., Henzi, S. P., Parker, J. L., Pollet, T. V, \& Barrett, L. (2017). The 'strength of weak ties' among female baboons: fitness-related benefits of social bonds. Animal Behaviour, 126, 101-106.

McKenna, A., Hanna, M., Banks, E., Sivachenko, A., Cibulskis, K., Kernytsky, A., Garimella, K., Altshuler, D., Gabriel, S., \& Daly, M. (2010). The genome analysis toolkit: a mapreduce framework for analyzing next-generation DNA sequencing data. Genome Research, 20(9), 1297-1303.

McLaren, W., Gil, L., Hunt, S. E., Riat, H. S., Ritchie, G. R. S., Thormann, A., Flicek, P., \& Cunningham, F. (2016). The ensembl variant effect predictor. Genome Biology, 17(1), 122.

Meigs, J. B., Shrader, P., Sullivan, L. M., McAteer, J. B., Fox, C. S., Dupuis, J., Manning, A. K., Florez, J. C., Wilson, P. W. F. \& D'Agostino Sr, R. B. (2008). Genotype score in addition to common risk factors for prediction of type 2 diabetes. New England Journal of Medicine, 359(21), 2208-2219.

Mekli, K., Payton, A., Miyajima, F., Platt, H., Thomas, E., Downey, D., Lloyd-Williams, K., Chase, D., Toth, Z. G., Elliott, R., Ollier, W. E., Anderson, I. M., Deakin, J. F. W., Bagdy, G., \& Juhasz, G. (2011). The HTR1A and HTR1B receptor genes influence stress-related information processing. European Neuropsychopharmacology, 21(1), 129-139.

Mell, H., Josserand, R., Decencière, B., Artacho, P., Meylan, S., \& Le Galliard, J.-F. (2016). Do personalities co-vary with metabolic expenditure and glucocorticoid stress response in adult lizards? Behavioral Ecology and Sociobiology, 70(6), 951961.

Mesiano, S., \& Jaffe, R. B. (1997). Developmental and functional biology of the primate fetal adrenal cortex. Endocrine Reviews, 18(3), 378-403.

Meyer, J. S., \& Hamel, A. F. (2014). Models of stress in nonhuman primates and their relevance for human psychopathology and endocrine dysfunction. ILAR Journal, $55(2), 347-360$.

Meyer, M., \& Kircher, M. (2010). Illumina sequencing library preparation for highly multiplexed target capture and sequencing. Cold Spring Harbor Protocols, 2010(6), pdb.prot5448.

Mickey, B. J., Zhou, Z., Heitzeg, M. M., Heinz, E., Hodgkinson, C. A., Hsu, D. T., Langenecker, S. A., Love, T. M., Pecina, M., Shafir, T., Stohler, C. S., Goldman, D., Zubieta, J. K., Peciña, M., \& Shafir, T. (2011). Emotion processing, major depression, and functional genetic variation of neuropeptide Y. Archives of General Psychiatry, 68(2), 158-166.

Mier, D., Kirsch, P., \& Meyer-Lindenberg, A. (2010). Neural substrates of pleiotropic 
action of genetic variation in COMT: a meta-analysis. Molecular Psychiatry, 15(9), 918-927.

Miles, D. R., \& Carey, G. (1997). Genetic and environmental architecture on human aggression. Journal of Personality and Social Psychology, 72(1), 207-217.

Miller-Butterworth, C. M., Kaplan, J. R., Shaffer, J., Devlin, B., Manuck, S. B., \& Ferrell, R. E. (2008). Sequence variation in the primate dopamine transporter gene and its relationship to social dominance. Molecular Biology and Evolution, 25(1), 18-28.

Miller, G. M., Bendor, J., Tiefenbacher, S., Yang, H., Novak, M. A., \& Madras, B. K. (2004). A mu-opioid receptor single nucleotide polymorphism in rhesus monkey: association with stress response and aggression. Molecular Psychiatry, 9(1), 99-108.

Mills, M. C., \& Tropf, F. C. (2015). The biodemography of fertility: a review and future research frontiers. Kölner Zeitschrift Für Soziologie Und Sozialpsychologie, 67(1), 397-424.

Minge, C., Berghänel, A., Schülke, O., \& Ostner, J. (2016). Patterns and consequences of male-infant relationships in wild Assamese macaques (Macaca assamensis). International Journal of Primatology, 37(3), 350-370.

Mitani, J. C. (2009). Male chimpanzees form enduring and equitable social bonds. Animal Behaviour, 77, 633-640.

Mitani, J. C., Merriwether, D. A., \& Zhang, C. (2000). Male affiliation, cooperation and kinship in wild chimpanzees. Animal Behaviour, 59(4), 885-893.

Mitchell, G. (1979). Behavioral sex differences in nonhuman primates. New York: Van Nostrand Reinhold.

Mitra, S. (2002). Diet and feeding behaviour of Assamese macaque (Macaca assamensis). Asian Primates, 8(1-2), 12-14.

Mitra, S., \& Alfred, J. R. B. (2007). Reproductive behaviour of the Assamese macaque (Macaca assamensis) in Darjeeling district, India. Records of the Zoological Survey of India, 107(4), 33-42.

Molinoff, P. B., \& Axelrod, J. (1971). Biochemistry of catecholamines. Annual Review of Biochemistry, 40(1), 465-400.

Montiglio, P.-O., Ferrari, C., \& Réale, D. (2013). Social niche specialization under constraints: personality, social interactions and environmental heterogeneity. Philosophical Transactions of the Royal Society of London B: Biological Sciences, 368(1618), 20120343.

Moons, W. G., Way, B. M., \& Taylor, S. E. (2014). Oxytocin and vasopressin receptor polymorphisms interact with circulating neuropeptides to predict human emotional reactions to stress. Emotion, 14(3), 562-572.

Moore, A. J., Brodie III, E. D., \& Wolf, J. B. (1997). Interacting phenotypes and the evolutionary process: I. Direct and indirect genetic effects of social interactions. Evolution, 51(5), 1352-1362.

Morales, J. C., \& Melnick, D. J. (1998). Phylogenetic relationships of the macaques (Cercopithecidae: Macaca), as revealed by high resolution restriction site mapping 
of mitochondrial ribosomal genes. Journal of Human Evolution, 34(1), 1-23.

Morgan, D. J., Liblau, R., Scott, B., Fleck, S., McDevitt, H. O., Sarvetnick, N., Lo, D., \& Sherman, L. A. (1996). CD8 (+) T cell-mediated spontaneous diabetes in neonatal mice. The Journal of Immunology, 157(3), 978-983.

Morrison, A. C., Bare, L. A., Chambless, L. E., Ellis, S. G., Malloy, M., Kane, J. P., Pankow, J. S., Devlin, J. J., Willerson, J. T., \& Boerwinkle, E. (2007). Prediction of coronary heart disease risk using a genetic risk score: the atherosclerosis risk in communities study. American Journal of Epidemiology, 166(1), 28-35.

Morton, F. B., Weiss, A., Buchanan-Smith, H. M., \& Lee, P. C. (2015). Capuchin monkeys with similar personalities have higher-quality relationships independent of age, sex, kinship and rank. Animal Behaviour, 105, 163-171.

Moss, C. J., Croze, H., \& Lee, P. C. (2011). The Amboseli elephants: a long-term perspective on a long-lived mammal. Chicago: University of Chicago Press.

Möstl, E., Maggs, J. L., Schrötter, G., Besenfelder, U., \& Palme, R. (2002). Measurements of cortisol metabolites in faeces of ruminants. Veterinary Research Communications, 26, 127-139.

Mueller, J. C., Lõhmussaar, E., Mägi, R., Remm, M., Bettecken, T., Lichtner, P., Biskup, S., Illig, T., Pfeufer, A., Luedemann, J., Schreiber, S., Pramstaller, P., Pichler, I., Romeo, G., Gaddi, A., Testa, A., Wichmann, H.-E., Metspalu, A., \& Meitinger, T. (2005). Linkage disequilibrium patterns and tagSNP transferability among European populations. American Journal of Human Genetics, 76(3), 387-98.

Muller, M. N., \& Wrangham, R. W. (2004a). Dominance, cortisol and stress in wild chimpanzees (Pan troglodytes schweinfurthii). Behavioral Ecology and Sociobiology, 55(4), 332-340.

Müller, N., Heistermann, M., Strube, C., Schülke, O., \& Ostner, J. (2017). Age, but not anthelmintic treatment, is associated with urinary neopterin levels in semi-free ranging Barbary macaques. Scientific Reports, 7, 41973.

Müller, N., Ostner, J., Schülke, O., \& Walter, L. (2014). Towards the non-invasive assessment of MHC genotype in wild primates: analysis of wild Assamese macaque MHC-DRB from fecal samples. American Journal of Primatology, 76(3), 230-238.

Munck, A., Guyre, P. M., \& Holbrook, N. J. (1984). Physiological functions of glucocorticoids in stress and their relation to pharmacological actions. Endocrine Reviews, 5(1), 25-44.

Muráni, E., Ponsuksili, S., D’Eath, R. B., Turner, S. P., Kurt, E., Evans, G., Tholking, L., Klont, R., Foury, A., Mormede, P., \& Wimmers, K. (2010). Association of HPA axis-related genetic variation with stress reactivity and aggressive behaviour in pigs. BMC Genetics, 11, 74.

Murugan, K., Babu, K., Sundaresan, R., Rajan, R., \& Sashital, D. G. (2017). The revolution continues: newly discovered systems expand the CRISPR-Cas toolkit. Molecular Cell, 68(1), 15-25.

NCBI Resource Coordinators. (2017). Database Resources of the National Center for Biotechnology Information. Nucleic Acids Research, 45(1), 12-17. 
Nelsen, S. (2017). Macaque ethogram. Primate Products, Inc., www.enrichmentrecord.com 06.02.2018

Nelson, R. J. (2011). An introduction to behavioral endocrinology. Sunderland: Sinauer Associates.

Nemeth, M., Pschernig, E., Wallner, B., \& Millesi, E. (2016). Non-invasive cortisol measurements as indicators of physiological stress responses in guinea pigs. PeerJ, 4, e1590.

Newman, T. K., Syagailo, Y. V., Barr, C. S., Wendland, J. R., Champoux, M., Graessle, M., Suomi, S. J., Higley, J. D., \& Lesch, K.-P. P. (2005). Monoamine oxidase A gene promoter variation and rearing experience influences aggressive behavior in rhesus monkeys. Biological Psychiatry, 57(2), 167-172.

Nicolson, N. A. (2008). Measurement of cortisol. In Luecken, L. J., \& Gallo, L. C. (Eds.), Handbook of physiological research methods in health psychology, 37-73. Newbury Park: Sage Publications Inc.

Niewenhuis, R., Pelzer, B., \& te Grotenhuis, M. (2013). Tools for detecting influential data in mixed effects models. $R$ Journal, 4(2), 38-47.

Nikolova, Y. S., Ferrell, R. E., Manuck, S. B., \& Hariri, A. R. (2011). Multilocus genetic profile for dopamine signaling predicts ventral striatum reactivity. Neuropsychopharmacology, 36(9), 1940-1947.

Nobel Media AB. (2014). The nobel prize in physiology or medicine 1950. Nobel Media $A B$, www.nobelprize.org 20.12.2017

Nsubuga, A. M., Robbins, M. M., Roeder, A. D., Morin, P. A., Boesch, C., \& Vigilant, L. (2004). Factors affecting the amount of genomic DNA extracted from ape faeces and the identification of an improved sample storage method. Molecular Ecology, 13(7), 2089-2094.

O'hara, R., Schröder, C. M., Mahadevan, R., Schatzberg, A. F., Lindley, S., Fox, S., Weiner, M., Kraemer, H. C., Noda, A., \& Lin, X. (2007). Serotonin transporter polymorphism, memory and hippocampal volume in the elderly: association and interaction with cortisol. Molecular Psychiatry, 12(6), 544-555.

Oldehinkel, A. J., Hartman, C. A., Nederhof, E., Riese, H., \& Ormel, J. (2011). Effortful control as predictor of adolescents' psychological and physiological responses to a social stress test: the tracking adolescents' individual lives survey. Development and Psychopathology, 23(2), 679-688.

Ostner, J., \& Schülke, O. (2014). The evolution of social bonds in primate males. Behaviour, 151, 871-906.

Ostner, J., \& Schülke, O. (2018). Linking sociality to fitness in primates: a call for mechanisms. Advances in the Study of Behavior, 50, 127-175.

Ostner, J., Heistermann, M., \& Schülke, O. (2008a). Dominance, aggression and physiological stress in wild male Assamese macaques (Macaca assamensis). Hormones and Behavior, 54(5), 613-619.

Ostner, J., Kappeler, P., \& Heistermann, M. (2008b). Androgen and glucocorticoid levels reflect seasonally occurring social challenges in male redfronted lemurs (Eulemur 
fulvus rufus). Behavioral Ecology and Sociobiology, 62(4), 627-638.

Ostner, J., Heistermann, M., \& Schülke, O. (2011). Male competition and its hormonal correlates in Assamese macaques (Macaca assamensis). Hormones and Behavior, 59(1), 105-113.

Ostner, J., Vigilant, L., Bhagavatula, J., Franz, M., \& Schülke, O. (2013). Stable heterosexual associations in a promiscuous primate. Animal Behaviour, 86(3), 623631.

Ostner, J., Young, C., \& Schülke, O. (2014). How males form coalitions against group rivals and the Pandit/van Schaik coalition model. Behaviour, 151(7), 907-934.

Øverli, Ø., Kotzian, S., \& Winberg, S. (2002). Effects of cortisol on aggression and locomotor activity in rainbow trout. Hormones and Behavior, 42(1), 53-61.

Padgett, D. A., \& Glaser, R. (2003). How stress influences the immune response. Trends in Immunology, 24(8), 444-448.

Pagliaccio, D., Luby, J. L., Bogdan, R., Agrawal, A., Gaffrey, M. S., Belden, A. C., Botteron, K. N., Harms, M. P., \& Barch, D. M. (2015). Amygdala functional connectivity, HPA axis genetic variation, and life stress in children and relations to anxiety and emotion regulation. Journal of Abnormal Psychology, 124(4), 817-833.

Palmatier, M. A., Kang, A. M., \& Kidd, K. K. (1999). Global variation in the frequencies of functionally different catechol-O-methyltransferase alleles. Biological Psychiatry, 46(4), 557-567.

Palme, R., \& Möstl, E. (1997). Measurement of cortisol metabolites in faeces of sheep as a parameter of cortosol concentration in blood. International Journal of Mammalian Biology, 62, 192-197.

Pandey, B. P., \& Chalise, M. K. (2015). General ecology \& time budgeting for Assamese monkey (Macaca assamensis) in Shivapuri Nagarjun National Park, Nepal. Biodiversity Conservation Efforts in Nepal, 1(2072), 57-73.

Papiol, S., Arias, B., Gastó, C., Gutiérrez, B., Catalán, R., \& Fañanás, L. (2007). Genetic variability at HPA axis in major depression and clinical response to antidepressant treatment. Journal of Affective Disorders, 104(1-3), 83-90.

Paquin, S., Lacourse, E., Brendgen, M., Vitaro, F., Dionne, G., Tremblay, R. E., \& Boivin, M. (2014). The genetic-environmental architecture of proactive and reactive aggression throughout childhood. Monatsschrift Für Kriminologie Und Strafrechtsform, 97, 398-420.

Parmigiani, S. (1986). Rank order in pairs of communally nursing female mice (Mus musculus domesticus) and maternal aggression towards conspecific intruders of differing sex. Aggressive Behavior, 12(5), 377-386.

Paschek, N., Müller, N., Heistermann, M., Ostner, J., \& Schülke, O. (in press). Subtypes of aggression and their relation to anxiety in Barbary macaques at Affenberg Salem. Aggressive Behavior.

Pasqualini, J. R., Lowy, J., Wiqvist, N., \& Diczfalusy, E. (1968). Biosynthesis of cortisol from $3 \beta, 17 \alpha, 21$-trihydroxypregn-5-en-20-one by the intact human foetus at midpregnancy. Biochimica et Biophysica Acta (BBA) - Lipids and Lipid Metabolism, 
152(3), 648-650.

Paudel, P. K., \& Chalise, M. K. (2017). General behavior and vegetation associated with the habitats of Assamese monkey (Macaca assamensis McClelland, 1840) along Kaligandaki river bank, western Nepal. Journal of Institute of Science and Technology, 22(1), 110-119.

Pavlov, K. A., Chistiakov, D. A., \& Chekhonin, V. P. (2012). Genetic determinants of aggression and impulsivity in humans. Journal of Applied Genetics, 53(1), 61-82.

Pearce, E., Wlodarski, R., Machin, A., \& Dunbar, R. I. M. (2017). Variation in the $\beta$ endorphin, oxytocin, and dopamine receptor genes is associated with different dimensions of human sociality. Proceedings of the National Academy of Sciences of the United States of America, 114(20), 5300-5305.

Penke, L., Denissen, J. J. A., \& Miller, G. F. (2007). The evolutionary genetics of personality. European Journal of Personality, 21(5), 549-587.

Penke, L., \& Jokela, M. (2016). The evolutionary genetics of personality revisited. Current Opinion in Psychology, 7, 104-109.

Perry, B. (2013). Where is the gender in behaviour genetics? The need for social epidemiology in research on gene-environment interactions. OA Genetics, 1(1), 8.

Perry, G. H. (2014). The promise and practicality of population genomics research with endangered species. International Journal of Primatology, 35(1), 55-70.

Perry, G. H., Marioni, J. C., Melsted, P., \& Gilad, Y. (2010). Genomic-scale capture and sequencing of endogenous DNA from feces. Molecular Ecology, 19(24), 53325344.

Pflieger, S., Lefebvre, V., \& Causse, M. (2001). The candidate gene approach in plant genetics: a review. Molecular Breeding, 7(4), 275-291.

Pflüger, L. S., Gutleb, D. R., Hofer, M., Fieder, M., Wallner, B., \& Steinborn, R. (2016). Allelic variation of the COMT gene in a despotic primate society: a haplotype is related to cortisol excretion in Macaca fuscata. Hormones and Behavior, 78, 220230 .

Phillips, K. A., Bales, K. L., Capitanio, J. P., Conley, A., Czoty, P. W., Hart, B. A., Hopkins, W. D., Hu, S.-L., Miller, L. A., \& Nader, M. A. (2014). Why primate models matter. American Journal of Primatology, 76(9), 801-827.

Plomin, R. (1994). Genetics and experience: The interplay between nature and nurture. Thousand Oaks: Sage Publications, Inc.

Plomin, R., \& Deary, I. J. (2015). Genetics and intelligence differences: five special findings. Molecular Psychiatry, 20(1), 98-108.

Plomin, R., DeFries, J. C., Knopik, V. S., \& Neiderheiser, J. (2013). Behavioral genetics. New York: Worth Publishers.

Plomin, R., DeFries, J. C., Knopik, V. S., \& Neiderhiser, J. M. (2016). Top 10 replicated findings from behavioral genetics. Perspectives on Psychological Science, 11(1), 323.

Porter, R. J., Gallagher, P., Watson, S., \& Young, A. H. (2004). Corticosteroid-serotonin 
interactions in depression: a review of the human evidence. Psychopharmacology, 173(1-2), 1-17.

Preis, A., Samuni, L., Mielke, A., Deschner, T., Crockford, C., \& Wittig, R. M. (2018). Urinary oxytocin levels in relation to post-conflict affiliations in wild male chimpanzees (Pan troglodytes verus). Hormones and Behavior, 105, 28-40.

Pressman, S. D., Cohen, S., Miller, G. E., Barkin, A., Rabin, B. S., \& Treanor, J. J. (2005). Loneliness, social network size, and immune response to influenza vaccination in college freshmen. Health Psychology, 24(3), 297-306.

Pride, R. E. (2005). Optimal group size and seasonal stress in ring-tailed lemurs (Lemur catta). Behavioral Ecology, 16(3), 550-560.

Puciłowski, O., Kozak, W., \& Valzelli, L. (1986). Effect of 6-OHDA injected into the locus coeruleus on apomorphine-induced aggression. Pharmacology Biochemistry and Behavior, 24(3), 773-775.

Purcell, S. M., Wray, N. R., Stone, J. L., Visscher, P. M., O’donovan, M. C., Sullivan, P. F., Sklar, P., Ruderfer, D. M., McQuillin, A., \& Morris, D. W. (2009). Common polygenic variation contributes to risk of schizophrenia and bipolar disorder. Nature, 460(7256), 748-752.

Qayyum, A., Zai, C. C., Hirata, Y., Tiwari, A. K., Cheema, S., Nowrouzi, B., Beitchman, J. H., \& Kennedy, J. L. (2015). The role of the catechol-o-methyltransferase (COMT) gene Val158Met in aggressive behavior, a review of genetic studies. Current Neuropharmacology, 13(6), 802-814.

Quednow, B. B., Schmechtig, A., Ettinger, U., Petrovsky, N., Collier, D. A., Vollenweider, F. X., Vollenweider, F. X., Wagner, M., \& Kumari, V. (2009). Sensorimotor gating depends on polymorphisms of the serotonin-2A receptor and catechol-O-methyltransferase, but not on neuregulin-1 Arg38Gln genotype: a replication study. Biological Psychiatry, 66(6), 614-620.

Queller, D. C., Strassmann, J. E., \& Hughes, C. R. (1993). Microsatellites and kinship. Trends in Ecology \& Evolution, 8(8), 285-288.

Ratey, J. J., \& Gordon, A. (1992). The psychopharmacology of aggression: toward a new day. Psychopharmacology Bulletin, 29(1), 65-73.

Reeder, D. M., \& Kramer, K. M. (2005). Stress in free-ranging mammals: integrating physiology, ecology, and natural history. Journal of Mammalogy, 86(2), 225-235.

Reif, A., Rösler, M., Freitag, C. M., Schneider, M., Eujen, A., Kissling, C., Wenzler, D., Jacob, C. P., Retz-Junginger, P., Thome, J., Lesch, K.-P., \& Retz, W. (2007). Nature and nurture predispose to violent behavior: serotonergic genes and adverse childhood environment. Neuropsychopharmacology, 32(11), 2375-2383.

Reiss, D. (2010). Genetic thinking in the study of social relationships: five points of entry. Perspectives on Psychological Science, 5(5), 502-515.

Rhee, S. H., \& Waldman, I. D. (2002). Genetic and environmental influences on antisocial behavior: a meta-analysis of twin and adoption studies. Psychological Bulletin, 128(3), 490-529.

Richter, C., Heesen, M., Nenadic, O., Ostner, J., \& Schülke, O. (2016). Males matter: 
increased home range size is associated with the number of resident males after controlling for ecological factors in wild Assamese macaques. American Journal of Physical Anthropology, 159(1), 52-62.

Riese, H., Rijsdijk, F. V, Rosmalen, J. G. M., Snieder, H., \& Ormel, J. (2009). Neuroticism and morning cortisol secretion: both heritable, but no shared genetic influences. Journal of Personality, 77(5), 1561-1576.

Rietschel, L., Streit, F., Zhu, G., McAloney, K., Frank, J., Couvy-Duchesne, B., Witt, S. H., Binz, T. M., McGrath, J., \& Hickie, I. B. (2017). Hair cortisol in twins: heritability and genetic overlap with psychological variables and stress-system genes. Scientific Reports, 7, 15351.

Ripatti, S., Tikkanen, E., Orho-Melander, M., Havulinna, A. S., Silander, K., Sharma, A., Guiducci, C., Perola, M., Jula, A., \& Sinisalo, J. (2010). A multilocus genetic risk score for coronary heart disease: case-control and prospective cohort analyses. The Lancet, 376(9750), 1393-1400.

Roberts, S. G. (2010). Constraints on social networks. Proceedings of the British Academy, 158, 115-134.

Roberts, S. G. B., Dunbar, R. I. M., Pollet, T. V., \& Kuppens, T. (2009). Exploring variation in active network size: constraints and ego characteristics. Social Networks, $31(2), 138-146$.

Robinson, G. E., Fernald, R. D., \& Clayton, D. F. (2008). Genes and social behavior. Science, 322(5903), 896-900.

Roeder, A. D., Archer, F. I., Poinar, H. N., \& Morin, P. A. (2004). A novel method for collection and preservation of faeces for genetic studies. Molecular Ecology Notes, 4(4), 761-764.

Rogers, J. (2018). The behavioral genetics of nonhuman primates: status and prospects. American Journal of Physical Anthropology, 165(65), 23-36.

Rogers, J., \& Gibbs, R. A. (2014). Comparative primate genomics: emerging patterns of genome content and dynamics. Nature Reviews Genetics, 15(5), 347-359.

Rohland, N., Harney, E., Mallick, S., Nordenfelt, S., \& Reich, D. (2015). Partial uracilDNA-glycosylase treatment for screening of ancient DNA. Philosophical Transactions of the Royal Society B, 370(1660), 20130624.

Rosado, B., García-Belenguer, S., León, M., Chacón, G., Villegas, A., \& Palacio, J. (2010). Blood concentrations of serotonin, cortisol and dehydroepiandrosterone in aggressive dogs. Applied Animal Behaviour Science, 123(3-4), 124-130.

Rosmond, R., Chagnon, Y. C., Holm, G., Chagnon, M., Pérusse, L., Lindell, K., Carlsson, B., Bouchard, C., \& Björntorp, P. (2000). A glucocorticoid receptor gene marker is associated with abdominal obesity, leptin, and dysregulation of the hypothalamicpituitary-adrenal axis. Obesity, 8(3), 211-218.

Roubertoux, P. L., Le Roy, I., Mortaud, S., Perez-Diaz, F., \& Tordjman, S. (1999). Measuring aggression in the mouse. Techniques in the Behavioral and Neural Sciences, 13, 696-709.

Rowell, T. E. (1971). Organization of caged groups of Cercopithecus monkeys. Animal 
Behaviour, 19(4), 625-645.

Rowell, T. E., \& Olson, D. K. (1983). Alternative mechanisms of social organization in monkeys. Behaviour, 86(1), 31-54.

Roy, A., Gorodetsky, E., Yuan, Q., Goldman, D., \& Enoch, M.-A. (2010). Interaction of FKBP5, a stress-related gene, with childhood trauma increases the risk for attempting suicide. Neuropsychopharmacology, 35(8), 1674-1683.

Roy, A., Hodgkinson, C. A., DeLuca, V., Goldman, D., \& Enoch, M.-A. (2012). Two HPA axis genes, CRHBP and FKBP5, interact with childhood trauma to increase the risk for suicidal behavior. Journal of Psychiatric Research, 46(1), 72-79.

Rutberg, A. T., \& Greenberg, S. A. (1990). Dominance, aggression frequencies and modes of aggressive competition in feral pony mares. Animal Behaviour, 40(2), 322-331.

Rutter, M., Moffitt, T. E., \& Caspi, A. (2006). Gene-environment interplay and psychopathology: multiple varieties but real effects. Journal of Child Psychology and Psychiatry, 47(3-4), 226-261.

Sabbatini, G., Vizioli, A. D. B., Visalberghi, E., \& Schino, G. (2012). Food transfers in capuchin monkeys: an experiment on partner choice. Biology Letters, 8(5), 757-759.

Salas, M., Temple, D., Abáigar, T., Cuadrado, M., Delclaux, M., Enseñat, C., Almagro, V., Martínez-Nevado, E., Quevedo, M., Carbajal, A., Tallo-Parra, O., Sabés-Alsina, M., Amat, M., Lopez-Bejar, M., Fernández-Bellon, H., \& Manteca, X. (2016). Aggressive behavior and hair cortisol levels in captive dorcas gazelles (Gazella dorcas) as animal-based welfare indicators. Zoo Biology, 35(6), 467-473.

Samorodnitsky, E., Jewell, B. M., Hagopian, R., Miya, J., Wing, M. R., Lyon, E., Damodaran, S., Bhatt, D., Reeser, J. W., \& Datta, J. (2015). Evaluation of hybridization capture versus amplicon-based methods for whole-exome sequencing. Human Mutation, 36(9), 903-914.

Sanchez, M. M., McCormack, K. M., \& Howell, B. R. (2015). Social buffering of stress responses in nonhuman primates: maternal regulation of the development of emotional regulatory brain circuits. Social Neuroscience, 10(5), 512-526.

Sapolsky, R. M. (1992). Cortisol concentrations and the social significance of rank instability among wild baboons. Psychoneuroendocrinology, 17(6), 701-709.

Sapolsky, R. M. (2005). The influence of social hierarchy on primate health. Science, 308(5722), 648-652.

Sapolsky, R. M., Romero, L. M., \& Munck, A. U. (2000). How do glucocorticoids influence stress responses? Integrating permissive, suppressive, stimulatory, and preparative actions. Endocrine Reviews, 21(1), 55-89.

Sarkar, P. (2014a). Assamese macaque: ecology and social dynamics. Saarbrücken: Lambert Academic Publishing.

Sarkar, P. (2014b). Does provisioning initiate more grooming interaction in primates: a study on Assamese macaque. Journal of Biodiversity and Environmental Sciences, 4(3), 211-219. 
Sarkar, P., Srivastava, A., Dasgupta, S., \& Bhattachar, P. C. (2012). Activity profile of free ranging forest group of Assamese macaque. The Clarion, 1(2), 59-67.

Satkoski-Trask, J. A., Garnica, W. T., Smith, D. G., Houghton, P., Lerche, N., \& Kanthaswamy, S. (2013). Single-nucleotide polymorphisms reveal patterns of allele sharing across the species boundary between rhesus (Macaca mulatta) and cynomolgus (M. fascicularis) macaques. American Journal of Primatology, 75(2), $135-144$.

Savitz, J., Hodgkinson, C. A., Martin-Soelch, C., Shen, P.-H., Szczepanik, J., Nugent, A., Herscovitch, P., Grace, A. A., Goldman, D., \& Drevets, W. C. (2013). The functional DRD3 Ser9Gly polymorphism (rs6280) is pleiotropic, affecting reward as well as movement. PLOS ONE, 8(1), e54108.

Schaaf, M. J. M., \& Cidlowski, J. A. (2002). AUUUA motifs in the 3' UTR of human glucocorticoid receptor $\alpha$ and $\beta$ mRNA destabilize mRNA and decrease receptor protein expression. Steroids, 67(7), 627-636.

Schaschl, H., Huber, S., Schaefer, K., Windhager, S., Wallner, B., \& Fieder, M. (2015). Signatures of positive selection in the cis-regulatory sequences of the human oxytocin receptor (OXTR) and arginine vasopressin receptor 1a (AVPR1A) genes. BMC Evolutionary Biology, 15(1), 85.

Schatz, S., \& Palme, R. (2001). Measurement of faecal cortisol metabolites in cats and dogs: a non-invasive method for evaluating adrenocortical function. Veterinary Research Communications, 25(4), 271-287.

Schatzberg, A. F., Keller, J., Tennakoon, L., Lembke, A., Williams, G., Kraemer, F. B., Sarginson, J. E., Lazzeroni, L. C., \& Murphy, G. M. (2014). HPA axis genetic variation, cortisol and psychosis in major depression. Molecular Psychiatry, 19(2), $220-227$.

Schino, G. (2001). Grooming, competition and social rank among female primates: a meta-analysis. Animal Behaviour, 62(2), 265-271.

Schino, G. (2007). Grooming and agonistic support: a meta-analysis of primate reciprocal altruism. Behavioral Ecology, 18(1), 115-120.

Schino, G., Ventura, R., \& Troisi, A. (2005). Grooming and aggression in captive Japanese macaques. Primates, 46(3), 207-209.

Schmid, V. S., \& de Vries, H. (2013). Finding a dominance order most consistent with a linear hierarchy: an improved algorithm for the I\&SI method. Animal Behaviour, 86(5), 1097-1105.

Schülke, O., Bhagavatula, J., Vigilant, L., \& Ostner, J. (2010). Social bonds enhance reproductive success in male macaques. Current Biology, 20(24), 2207-2210.

Schülke, O., Pesek, D., Whitman, B. J., \& Ostner, J. (2011). Ecology of Assamese macaques (Macaca assamensis) at Phu Khieo Wildlife Sanctuary, Thailand. Journal of Wildlife Thailand, 18, 1-15.

Schülke, O., Heistermann, M., \& Ostner, J. (2014). Lack of evidence for energetic costs of mate-guarding in wild male Assamese macaques (Macaca assamensis). International Journal of Primatology, 35(3-4), 677-700. 
Schulkin, J. (2011). Evolutionary conservation of glucocorticoids and corticotropin releasing hormone: behavioral and physiological adaptations. Brain Research, 1392, $27-46$.

Schwandt, M. L., Lindell, S. G., Chen, S., Higley, J. D., Suomi, S. J., Heilig, M., \& Barr, C. S. (2010). Alcohol response and consumption in adolescent rhesus macaques: life history and genetic influences. Alcohol, 44(1), 67-80.

Schwandt, M. L., Lindell, S. G., Higley, J. D., Suomi, S. J., Heilig, M., \& Barr, C. S. (2011). OPRM1 gene variation influences hypothalamic-pituitary-adrenal axis function in response to a variety of stressors in rhesus macaques. Psychoneuroendocrinology, 36(9), 1303-1311.

Schwandt, M. L., Lindell, S. G., Sjöberg, R. L., Chisholm, K. L., Higley, J. D., Suomi, S. J., Heilig, M., \& Barr, C. S. (2010). Gene-environment interactions and response to social intrusion in male and female rhesus macaques. Biological Psychiatry, 67(4), 323-330.

Scott, A. P., \& Ellis, T. (2007). Measurement of fish steroids in water-a review. General and Comparative Endocrinology, 153(1-3), 392-400.

Seeman, T. E. (1996). Social ties and health: the benefits of social integration. Annals of Epidemiology, 6(5), 442-451.

Seeman, T. E., Singer, B., Wilkinson, C. W., \& McEwen, B. (2001). Gender differences in age-related changes in HPA axis reactivity. Psychoneuroendocrinology, 26(3), $225-240$.

Seltzer, L. J., Ziegler, T. E., \& Pollak, S. D. (2010). Social vocalizations can release oxytocin in humans. Proceedings of the Royal Society B: Biological Sciences, 277(1694), 2661-2666.

Seneviratne, C., Huang, W., Ait-Daoud, N., Li, M. D., \& Johnson, B. A. (2009). Characterization of a functional polymorphism in the 3' UTR of SLC6A4 and its association with drinking intensity. Alcoholism: Clinical and Experimental Research, 33(2), 332-339.

Sesack, S. R., Hawrylak, V. A., Matus, C., Guido, M. A., \& Levey, A. I. (1998). Dopamine axon varicosities in the prelimbic division of the rat prefrontal cortex exhibit sparse immunoreactivity for the dopamine transporter. The Journal of Neuroscience, 18(7), 2697-2708.

Seyfarth, R. M. (1977). A model of social grooming among adult female monkeys. Journal of Theoretical Biology, 65(4), 671-698.

Seyfarth, R. M., \& Cheney, D. L. (2012). The evolutionary origins of friendship. Annual Review of Psychology, 63, 153-177.

Seyfarth, R. M., Silk, J. B., \& Cheney, D. L. (2012). Variation in personality and fitness in wild female baboons. Proceedings of the National Academy of Sciences of the United States of America, 109(42), 16980-16985.

Seyfarth, R. M., Silk, J. B., \& Cheney, D. L. (2014). Social bonds in female baboons: the interaction between personality, kinship and rank. Animal Behaviour, 87, 23-29.

Shanahan, M. J., \& Hofer, S. M. (2011). Molecular genetics, aging, and well-being: 
sensitive period, accumulation, and pathway models. In Binstock, R. H., \& George, L. K. (Eds.), Handbook of aging and the social sciences, 135-147. London: Academic Press.

Shattuck, M. (2011). The molecular evolution of the serotonin system in macaques (Macaca): a detailed survey of four serotonin-related genes. Dissertation University of Illinois at Urbana-Champaign.

Shattuck, M. R., Satkoski-Trask, J., Deinard, A., Tito, R. Y., Smith, D. G., Melnick, D. J., \& Malhi, R. S. (2014). Patterns of genetic variation and the role of selection in HTR1A and HTR1B in macaques (Macaca). BMC Genetics, 15(1), 1-13.

Silk, J. B. (1982). Altruism among female Macaca radiata: explanations and analysis of patterns of grooming and coalition formation. Behaviour, 79(2), 162-188.

Silk, J. B. (2002). Using the 'F'-word in primatology. Behaviour, 139(2), 421-446.

Silk, J. B., Alberts, S. C., \& Altmann, J. (2003). Social bonds of female baboons enhance infant survival, 302(5648), 1231-1234.

Silk, J. B., Altmann, J., \& Alberts, S. C. (2006a). Social relationships among adult female baboons (Papio cynocephalus) I. Variation in the strength of social bonds, Behavioral Ecology and Sociobiology 61(2), 183-195.

Silk, J. B., Altmann, J., Alberts, S. C. (2006b). Social relationships among adult female baboons (Papio cynocephalus) II. Variation in the quality and stability of social bonds. Behavioral Ecology and Sociobiology, 61(2), 197-204.

Silk, J. B., Beehner, J. C., Bergman, T. J., Crockford, C., Engh, A. L., Moscovice, L. R., Wittig, R. M., Seyfarth, R. M., \& Cheney, D. L. (2009). The benefits of social capital: close social bonds among female baboons enhance offspring survival. Proceedings of the Royal Society B: Biological Sciences, 276(1670), 3099-3104.

Silk, J. B., Beehner, J. C., Bergman, T. J., Crockford, C., Engh, A. L., Moscovice, L. R., Wittig, R. M., Seyfarth, R. M., \& Cheney, D. L. (2010a). Female chacma baboons form strong, equitable, and enduring social bonds. Behavioral Ecology and Sociobiology, 64(11), 1733-1747.

Silk, J. B., Beehner, J. C., Bergman, T. J., Crockford, C., Engh, A. L., Moscovice, L. R., Wittig, R. M., Seyfarth, R. M., \& Cheney, D. L. (2010b). Strong and consistent social bonds enhance the longevity of female baboons. Current Biology, 20(15), 1359-1361.

Silverman, J. L., Yang, M., Lord, C., \& Crawley, J. N. (2010). Behavioural phenotyping assays for mouse models of autism. Nature Reviews Neuroscience, 11(7), 490-502.

Simons, R. L., Lei, M. K., Beach, S. R. H., Brody, G. H., Philibert, R. A., \& Gibbons, F. X. (2011). Social environment, genes, and aggression: evidence supporting the differential susceptibility perspective. American Sociological Review, 76(6), 883912.

Simpson, E. R., \& Waterman, M. R. (1988). Regulation of the synthesis of steroidogenic enzymes in adrenal cortical cells by ACTH. Annual Review of Physiology, 50(1), 427-440.

Singh, J. P., Volavka, J., Czobor, P., \& van Dorn, R. A. (2012). A meta-analysis of the 
Val158Met COMT polymorphism and violent behavior in schizophrenia. PLOS ONE, 7(8), e43423.

Skinner, S. W., \& Lockard, J. S. (1979). An ethogram of the liontail macaque (Macaca silenus) in captivity. Applied Animal Ethology, 5(3), 241-253.

Skuse, D. H., \& Gallagher, L. (2011). Genetic influences on social cognition. Pediatric Research, 69, 85-91.

Smith, J. M. (1974). The theory of games and the evolution of animal conflicts. Journal of Theoretical Biology, 47(1), 209-221.

Smith, T. F., \& Waterman, M. S. (1981). Comparison of biosequences. Advances in Applied Mathematics, 2(4), 482-489.

Smith, J. M. (1982). Evolution and the theory of games. Cambridge: Cambridge University Press.

Smith, J. M., \& Brown, R. L. W. (1986). Competition and body size. Theoretical Population Biology, 30(2), 166-179.

Smith, J. M, Harper, D. G. C., \& Brookfield, J. F. Y. (1988). The evolution of aggression: can selection generate variability? Philosophical Transactions of the Royal Society of London. Series B, Biological Sciences, 319(1196), 557-570.

Smith, G. W., Aubry, J.-M., Dellu, F., Contarino, A., Bilezikjian, L. M., Gold, L. H., Chen, R., Marchuk, Y., Hauser, C., \& Bentley, C. A. (1998). Corticotropin releasing factor receptor 1-deficient mice display decreased anxiety, impaired stress response, and aberrant neuroendocrine development. Neuron, 20(6), 1093-1102.

Smith, S. M., \& Vale, W. W. (2006). The role of the hypothalamic-pituitary-adrenal axis in neuroendocrine responses to stress. Dialogues in Clinical Neuroscience, 8(4), 383-395.

Smolka, M. N., Schumann, G., Wrase, J., Grüsser, S. M., Flor, H., Mann, K., Braus, D. F., Goldman, D., Büchel, C., \& Heinz, A. (2005). Catechol-O-methyltransferase val158met genotype affects processing of emotional stimuli in the amygdala and prefrontal cortex. Journal of Neuroscience, 25(4), 836-842.

Snyder-Mackler, N., Majoros, W. H., Yuan, M. L., Shaver, A. O., Gordon, J. B., Kopp, G. H., Schlebusch, S. A., Wall, J. D., Alberts, S. C., Mukherjee, S., Zhou, X., \& Tung, J. (2016a). Efficient genome-wide sequencing and low-coverage pedigree analysis from noninvasively collected samples. Genetics, 203(2), 699-714.

Snyder-Mackler, N., Sanz, J. J., Kohn, J. N., Brinkworth, J. F., Morrow, S., Shaver, A. O., Grenier, J.-C., Pique-Regi, R., Johnson, Z. P., Wilson, M. E., Barreiro, L. B., \& Tung, J. (2016b). Social status alters immune regulation and response to infection in macaques. Science, 354(6315), 1041-1045.

Snyder-Mackler, N., Kohn, J. N., Barreiro, L. B., Johnson, Z. P., Wilson, M. E., \& Tung, J. (2016c). Social status drives social relationships in groups of unrelated female rhesus macaques. Animal Behaviour, 111, 307-317.

Soma, K. K., Scotti, M.-A. L., Newman, A. E. M., Charlier, T. D., \& Demas, G. E. (2008). Novel mechanisms for neuroendocrine regulation of aggression. Frontiers in Neuroendocrinology, 29(4), 476-489. 
Song, S., Oh, D.-Y., Cho, G.-J., Kim, D. H., Park, Y.-S., \& Han, K. (2017). Targeted next-generation sequencing for identifying genes related to horse temperament. Genes \& Genomics, 39(12), 1325-1333.

Spycher, B. D., Henderson, J., Granell, R., Evans, D. M., Smith, G. D., Timpson, N. J., \& Sterne, J. A. C. (2012). Genome-wide prediction of childhood asthma and related phenotypes in a longitudinal birth cohort. Journal of Allergy and Clinical Immunology, 130(2), 503-509.

Staes, N., Stevens, J. M. G., Helsen, P., Hillyer, M., Korody, M., \& Eens, M. (2014). Oxytocin and vasopressin receptor gene variation as a proximate base for inter- and intraspecific behavioral differences in bonobos and chimpanzees. PLOS ONE, 9(11), $1-9$.

Stanley, C. R., \& Dunbar, R. I. M. (2013). Consistent social structure and optimal clique size revealed by social network analysis of feral goats, Capra hircus. Animal Behaviour, 85(4), 771-779.

Stefanis, N. C., Henquet, C., Avramopoulos, D., Smyrnis, N., Evdokimidis, I., MyinGermeys, I., Stefanis, C. N., \& van Os, J. (2007). COMT ValMet moderation of stress-induced psychosis. Psychological Medicine, 37(11), 1651-1656.

Stein, D. J., Newman, T. K., Savitz, J., \& Ramesar, R. (2006). Warriors versus worriers: the role of COMT gene variants. CNS Spectrums, 11(10), 745-748.

Stephens, S. B. Z., \& Wallen, K. (2013). Environmental and social influences on neuroendocrine puberty and behavior in macaques and other nonhuman primates. Hormones and Behavior, 64(2), 226-239.

Steptoe, A., van Jaarsveld, C. H. M., Semmler, C., Plomin, R., \& Wardle, J. (2009). Heritability of daytime cortisol levels and cortisol reactivity in children. Psychoneuroendocrinology, 34(2), 273-280.

Sternberg, S. H., \& Doudna, J. A. (2015). Expanding the biologist's toolkit with CRISPRCas9. Molecular Cell, 58(4), 568-574.

Street, S. L., Kyes, R. C., Grant, R., \& Ferguson, B. (2007). Single nucleotide polymorphisms (SNPs) are highly conserved in rhesus (Macaca mulatta) and cynomolgus (Macaca fascicularis) macaques. BMC Genomics, 8(1), 480.

Strous, R. D., Bark, N., Parsia, S. S., Volavka, J., \& Lachman, H. M. (1997). Analysis of a functional catechol-O-methyltransferase gene polymorphism in schizophrenia: evidence for association with aggressive and antisocial behavior. Psychiatry Research, 69(2-3), 71-77.

Strous, R. D., Nolan, K. a, Lapidus, R., Diaz, L., Saito, T., \& Lachman, H. M. (2003). Aggressive behavior in schizophrenia is associated with the low enzyme activity COMT polymorphism: a replication study. American Journal of Medical Genetics. Part B, Neuropsychiatric Genetics, 120(1), 29-34.

Subbannayya, T., Balakrishnan, L., Sudarshan, G., Advani, J., Kumar, S., Mahmood, R., Nair, B., Sirdeshmukh, R., Mukherjee, K. K., \& Umathe, S. N. (2013). An integrated map of corticotropin-releasing hormone signaling pathway. Journal of Cell Communication and Signaling, 7(4), 295-300.

Sueur, C., \& Petit, O. (2008). Organization of group members at departure is driven by 
social structure in Macaca. International Journal of Primatology, 29(4), 1085-1098.

Sukmak, M., Wajjwalku, W., Ostner, J., \& Schülke, O. (2014a). Dominance rank, female reproductive synchrony, and male reproductive skew in wild Assamese macaques. Behavioral Ecology and Sociobiology, 68(7), 1097-1108.

Sukmak, M., Malaivijitnond, S., Schülke, O., Ostner, J., Hamada, Y., \& Wajjwalku, W. (2014b). Preliminary study of the genetic diversity of eastern Assamese macaques (Macaca assamensis assamensis) in Thailand based on mitochondrial DNA and microsatellite markers. Primates, 55(2), 189-197.

Sukmak, M., Wajjwalku, W., Ostner, J., \& Schülke, O. (2017). A first report of noninvasive adenovirus detection in wild Assamese macaques in Thailand. Primates, 58(2), 307-313.

Tabor, H. K., Risch, N. J., \& Myers, R. M. (2002). Candidate-gene approaches for studying complex genetic traits: practical considerations. Nature Reviews Genetics, 3(5), 391-397.

Tadić, A., Victor, A., Başkaya, Ö., von Cube, R., Hoch, J., Kouti, I., Anicker, N. J., Höppner, W., Lieb, K., \& Dahmen, N. (2009). Interaction between gene variants of the serotonin transporter promoter region (5-HTTLPR) and catechol O-methyltransferase (COMT) in borderline personality disorder. American Journal of Medical Genetics Part B: Neuropsychiatric Genetics, 150(4), 487-495.

Takahashi, T., Babygirija, R. R., \& Ludwig, K. (2015). Anti-stress effect of hypothalamic oxytocin-Importance of somatosensory stimulation and social buffering. International Journal of Neurology Research, 1(3), 96-101.

Tata, P., Subramaniayan, K., Suresh, A., Gupta, V., Bahadur, U., \& Agrawal, N. (2017). Biologic basis of personalized therapy in head and neck squamous cell carcinoma. In Kuriakose, M. A. (Ed.), Contemporary Oral Oncology, 461-486. Cham: Springer.

Taylor, S. E., Klein, L. C., Lewis, B. P., Gruenewald, T. L., Gurung, R. A. R., \& Updegraff, J. A. (2000). Biobehavioral responses to stress in females: tend-andbefriend, not fight-or-flight. Psychological Review, 107(3), 411-429.

Tenhunen, J., Salminen, M., Lundström, K., Kiviluoto, T., Savolainen, R., \& Ulmanen, I. (1994). Genomic organization of the human catechol O-methyltransferase gene and its expression from two distinct promoters. The FEBS Journal, 223(3), 1049-1059.

Thapar, A., Langley, K., Fowler, T., Rice, F., Turic, D., Whittinger, N., Aggleton, J., van den Bree, M., Owen, M., \& O'Donovan, M. (2005). Catechol O-methyltransferase gene variant and birth weight predict early-onset antisocial behavior in children with attention-deficit/hyperactivity disorder. Archives of General Psychiatry, 62(11), $1275-1278$.

The IUCN Red List of Threatened Species. (2017). The IUCN Red List of Threatened Species 2017(3), www.iucnredlist.org. 05.01.2018

Thierry, B. (2000). Covariation of conflict management patterns across macaque species. In Aureli, F., \& de Waal, F. (Eds.), Natural conflict resolution, 106-128. Oakland: University of California Press.

Thierry, B. (2007). The macaques: A double-layered social organization. In Campbell, C., Fuentes, A., \& MacKinnon, K. (Eds.), Primates in Perspective, 224-239. Oxford: 
Oxford University Press.

Thierry, B., Aureli, F., Nunn, C. L., Petit, O., Abegg, C., \& de Waal, F. B. M. (2008). A comparative study of conflict resolution in macaques: insights into the nature of trait covariation. Animal Behaviour, 75(3), 847-860.

Thierry, B., Singh, M., \& Kaumanns, W. (2004). Macaque societies: a model for the study of social organization. Cambridge: Cambridge University Press.

Thoits, P. A. (2011). Mechanisms linking social ties and support to physical and mental health. Journal of Health and Social Behavior, 52(2), 145-161.

Thompson, J., Sonuga-Barke, E. J., Morgan, A. R., Cornforth, C. M., Turic, D., Ferguson, L. R., Mitchell, E. A., \& Waldie, K. E. (2012). The catechol-O-methyltransferase (COMT) Val158Met polymorphism moderates the effect of antenatal stress on childhood behavioural problems: longitudinal evidence across multiple ages. Developmental Medicine \& Child Neurology, 54(2), 148-154.

Thompson, N. A., \& Cords, M. (2018). Stronger social bonds do not always predict greater longevity in a gregarious primate. Ecology and Evolution, 8(3), 1604-1614.

Thomson, C. J., Carlson, S. R., \& Rupert, J. L. (2013). Association of a common D3 dopamine receptor gene variant is associated with sensation seeking in skiers and snowboarders. Journal of Research in Personality, 47(2), 153-158.

Thorsell, A., Carlsson, K., Ekman, R., \& Heilig, M. (1999). Behavioral and endocrine adaptation, and up-regulation of NPY expression in rat amygdala following repeated restraint stress. Neuroreport, 10(14), 3003-3007.

Tiddi, B., Aureli, F., Polizzi di Sorrentino, E., Janson, C. H., \& Schino, G. (2011). Grooming for tolerance? Two mechanisms of exchange in wild tufted capuchin monkeys. Behavioral Ecology, 22(3), 663-669.

Tidey, J. W., \& Miczek, K. A. (1996). Social defeat stress selectively alters mesocorticolimbic dopamine release: an in vivo microdialysis study. Brain Research, 721(1), 140-149.

Timm, K., Tilgar, V., \& Saag, P. (2015). DRD4 gene polymorphism in great tits: genderspecific association with behavioural variation in the wild. Behavioral Ecology and Sociobiology, 69(5), 729-735.

Tinbergen, N. (1963). On aims and methods of ethology. Ethology, 20(4), 410-433.

Tinklepaugh, O. L. (1931). Fur-picking in monkeys as an act of adornment. Journal of Mammalogy, 12(4), 430-431.

Tochigi, M., Kato, C., Otowa, T., Hibino, H., Marui, T., Ohtani, T., Umekage, T., Kato, N., \& Sasaki, T. (2006). Association between corticotropin-releasing hormone receptor 2 (CRHR2) gene polymorphism and personality traits. Psychiatry and Clinical Neurosciences, 60(4), 524-526.

Tosato, S., Bonetto, C., Di Forti, M., Collier, D., Cristofalo, D., Bertani, M., Zanoni, M., Marrella, G., Lazzarotto, L., \& Lasalvia, A. (2011). Effect of COMT genotype on aggressive behaviour in a community cohort of schizophrenic patients. Neuroscience Letters, 495(1), 17-21. 
Traylor, M., Rutten-Jacobs, L. C. A., Thijs, V., Holliday, E. G., Levi, C., Bevan, S., Malik, R., Boncoraglio, G., Sudlow, C., \& Rothwell, P. M. (2016). Genetic associations with white matter hyperintensities confer risk of lacunar stroke. Stroke, 47(5), 1174-1179.

Trefilov, A., Berard, J., Krawczak, M., \& Schmidtke, J. (2000). Natal dispersal in rhesus macaques is related to serotonin transporter gene promoter variation. Behavior Genetics, 30(4), 295-301.

Tucker-Drob, E. M., Briley, D. A., \& Harden, K. P. (2013). Genetic and environmental influences on cognition across development and context. Current Directions in Psychological Science, 22(5), 349-355.

Tucker-Drob, E. M., Grotzinger, A. D., Briley, D. A., Engelhardt, L. E., Mann, F. D., Patterson, M., Kirschbaum, C., Adam, E. K., Church, J. A., \& Tackett, J. L. (2017). Genetic influences on hormonal markers of chronic hypothalamic-pituitary-adrenal function in human hair. Psychological Medicine, 47(8), 1389-1401.

Tunbridge, E. M. (2010). The catechol-O-methyltransferase gene: its regulation and polymorphisms. International Review of Neurobiology, 95(1), 7-27.

Tung, J., Alberts, S. C., \& Wray, G. A. (2010). Evolutionary genetics in wild primates: combining genetic approaches with field studies of natural populations. Trends in Genetics , 26(8), 353-362.

Tung, J., Archie, E. A., Altmann, J., \& Alberts, S. C. (2016). Cumulative early life adversity predicts longevity in wild baboons. Nature Communications, 7, 11181.

Tung, J., Barreiro, L. B., Johnson, Z. P., Hansen, K. D., Michopoulos, V., Toufexis, D., Michelini, K., Wilson, M. E., \& Gilad, Y. (2012). Social environment is associated with gene regulatory variation in the rhesus macaque immune system. Proceedings of the National Academy of Sciences of the United States of America, 109(17), 64906495.

Turkheimer, E. (2000). Three laws of behavior genetics and what they mean. Current Directions in Psychological Science, 9(5), 160-164.

Turkheimer, E., \& Gottesman, I. I. (1991). Is H2=0 a null hypothesis anymore? Behavioral and Brain Sciences, 14(3), 410-411.

Tuvblad, C., \& Baker, L. A. (2011). Human aggression across the lifespan: genetic propensities and environmental moderators. Advances in Genetics, 75, 171-214.

Tuvblad, C., Raine, A., Zheng, M., \& Baker, L. A. (2009). Genetic and environmental stability differs in reactive and proactive aggression. Aggressive Behavior, 35(6), 437-452.

Tzeng, W.-Y., Huang, T.-Y., Cherng, C. G., Yang, S.-N., \& Yu, L. (2018). Social buffering prevents stress-induced decreases in dendritic length, branching in dentate granule cells and hippocampus-related memory performance. Neuropsychiatry, $8(1)$, 197-213.

Uchino, B. N. (2004). Social support and physical health: Understanding the health consequences of relationships. Yale: Yale University Press.

Uchino, B. N. (2006). Social support and health: a review of physiological processes 
potentially underlying links to disease outcomes. Journal of Behavioral Medicine, 29(4), 377-387.

Uchino, B. N., Cacioppo, J. T., \& Kiecolt-Glaser, J. K. (1996). The relationship between social support and physiological processes: a review with emphasis on underlying mechanisms and implications for health. Psychological Bulletin, 119(3), 488-531.

Ulbricht, J. A., \& Neiderhiser, J. M. (2009). Genotype-environment correlation and family relationships. In Kim, Y.-K. (Ed.), Handbook of Behavior Genetics, 209-221. New York: Springer

Untergasser, A., Cutcutache, I., Koressaar, T., Ye, J., Faircloth, B. C., Remm, M., \& Rozen, S. G. (2012). Primer3-new capabilities and interfaces. Nucleic Acids Research, 40(15), e115.

Utge, S., Räikkönen, K., Kajantie, E., Lipsanen, J., Andersson, S., Strandberg, T., Reynolds, R. M., Eriksson, J. G., \& Lahti, J. (2018). Polygenic risk score of SERPINA6/SERPINA1 associates with diurnal and stress-induced HPA axis activity in children. Psychoneuroendocrinology, 93, 1-7.

Vallender, E. J., \& Miller, G. M. (2013). Nonhuman primate models in the genomic era: a paradigm shift. ILAR Journal, 54(2), 154-165.

Vallender, E. J., Priddy, C. M., Hakim, S., Yang, H., Chen, G. L., \& Miller, G. M. (2008). Functional variation in the 3 untranslated region of the serotonin transporter in human and rhesus macaque. Genes, Brain and Behavior, 7(6), 690-697.

van Hooff, J. A. R. A. M., \& Wensing, J. A. B. (1987). Dominance and its behavioural measures in a captive wolf pack. In Frank, H. W. (Ed.), Perspectives in vertebrate science, Volume 4 Man and wolf, 219-252. Dordrecht: Junk Publishers.

van Hulle, C. A., Shirtcliff, E. A., Lemery-Chalfant, K., \& Goldsmith, H. H. (2012). Genetic and environmental influences on individual differences in cortisol level and circadian rhythm in middle childhood. Hormones and Behavior, 62(1), 36-42.

van Noordwijk, M. A., \& van Schaik, C. P. (2004). Sexual selection and the careers of primate males: paternity concentration, dominance-acquisition tactics and transfer decisions. In Kappeler, P. M., \& van Schaik, C. P. (Eds.), Sexual selection in primates - new and comparative perspectives, 208-229. Cambridge: Cambridge University Press.

van der Voorn, B., Hollanders, J. J., Ket, J. C., Rotteveel, J., \& Finken, M. J. (2017). Gender-specific differences in hypothalamus-pituitary-adrenal axis activity during childhood: a systematic review and meta-analysis. Biology of Sex Differences, 8(1), 3 .

van West, D., Del-Favero, J., Deboutte, D., van Broeckhoven, C., \& Claes, S. (2010). Associations between common arginine vasopressin $1 \mathrm{~b}$ receptor and glucocorticoid receptor gene variants and HPA axis responses to psychosocial stress in a child psychiatric population. Psychiatry Research, 179(1), 64-68.

van Winkel, R., Henquet, C., Rosa, A., Papiol, S., Faňanás, L., De Hert, M., Peuskens, J., van Os, J., \& Myin-Germeys, I. (2008). Evidence that the COMT(Val158Met) polymorphism moderates sensitivity to stress in psychosis: an experience-sampling study. American Journal of Medical Genetics Part B: Neuropsychiatric Genetics, 
147(1), 10-17.

Varghese, F. P., \& Brown, E. S. (2001). The hypothalamic-pituitary-adrenal axis in major depressive disorder: a brief primer for primary care physicians. Primary Care Companion to The Journal of Clinical Psychiatry, 3(4), 151-155.

Varley, M., \& Symmes, D. (1966). The hierarchy of dominance in a group of macaques. Behaviour, 27(1), 54-74.

Vassos, E., Collier, D. A., \& Fazel, S. (2014). Systematic meta-analyses and field synopsis of genetic association studies of violence and aggression. Molecular Psychiatry, 19(4), 471-477.

Velders, F. P., Kuningas, M., Kumari, M., Dekker, M. J., Uitterlinden, A. G., Kirschbaum, C., Hek, K., Hofman, A., Verhulst, F. C., Kivimaki, M., van Duijn, C. M., Walker, B. R., \& Tiemeier, H. (2011). Genetics of cortisol secretion and depressive symptoms: a candidate gene and genome wide association approach. Psychoneuroendocrinology, 36(7), 1053-1061.

Venter, J. C., Adams, M. D., Myers, E. W., Li, P. W., Mural, R. J., Sutton, G. G., Smith, H. O., Yandell, M., Evans, C. A., \& Holt, R. A. (2001). The sequence of the human genome. Science, 291(5507), 1304-1351.

Vidgren, J., Svensson, L. A., \& Liljas, A. (1994). Crystal structure of catechol Omethyltransferase. Nature, 368, 354-358.

Vijayraghavan, S., Wang, M., Birnbaum, S. G., Williams, G. V., \& Arnsten, A. F. T. (2007). Inverted-U dopamine D1 receptor actions on prefrontal neurons engaged in working memory. Nature Neuroscience, 10(3), 376-384.

Volavka, J. (2002). Neurobiology of violence. Washington: American Psychiatric Association Publishing.

Volavka, J., Bilder, R., \& Nolan, K. (2004). Catecholamines and aggression: the role of COMT and MAO polymorphisms. Annals of the New York Academy of Sciences, 1036, 393-398.

von Dawans, B., Kirschbaum, C., \& Heinrichs, M. (2011). The trier social stress test for groups (TSST-G): a new research tool for controlled simultaneous social stress exposure in a group format. Psychoneuroendocrinology, 36(4), 514-522.

Vrieze, S. I., McGue, M., \& Iacono, W. G. (2012). The interplay of genes and adolescent development in substance use disorders: leveraging findings from GWAS metaanalyses to test developmental hypotheses about nicotine consumption. Human Genetics, 131(6), 791-801.

Wagner, S., Baskaya, Ö., Anicker, N. J., Dahmen, N., Lieb, K., \& Tadić, A. (2010). The catechol o-methyltransferase (COMT) val(158)met polymorphism modulates the association of serious life events (SLE) and impulsive aggression in female patients with borderline personality disorder (BPD). Acta Psychiatrica Scandinavica, 122(2), $110-117$.

Walder, D. J., Trotman, H. D., Cubells, J. F., Brasfield, J., Tang, Y., \& Walker, E. F. (2010). Catechol-O-Methyltransferase (COMT) modulation of cortisol secretion in psychiatrically at-risk and healthy adolescents. Psychiatric Genetics, 20(4), 166170. 
Walker, E., Mittal, V., \& Tessner, K. (2008). Stress and the hypothalamic pituitary adrenal axis in the developmental course of schizophrenia. Annual Review of Clinical Psychology, 4, 189-216.

Wang, J., Qin, W., Liu, B., Zhou, Y., Wang, D., Zhang, Y., Jiang, T., \& Yu, C. (2014). Neural mechanisms of oxytocin receptor gene mediating anxiety-related temperament. Brain Structure \& Function, 219(5), 1543-1554.

Wang, Y., Yang, Y., Hui, L., Tie, C., Li, F., Xu, Z.-Q. D., \& Wang, C. (2013). A neuropeptide $\mathrm{Y}$ variant (rs16139) associated with major depressive disorder in replicate samples from chinese han population. PLOS ONE, 8(2), e57042.

Wasser, S. K., Hunt, K. E., Brown, J. L., Cooper, K., Crockett, C. M., Bechert, U., Millspaugh, J. J., Larson, S., \& Monfort, S. L. (2000). A generalized fecal glucocorticoid assay for use in a diverse array of nondomestic mammalian and avian species. General and Comparative Endocrinology, 120(3), 260-75.

Wasserman, D., \& Wachbroit, R. (2001). Genetics and criminal behavior. Cambridge: Cambridge University Press.

Wasserman, D., Wasserman, J., \& Sokolowski, M. (2010). Genetics of HPA-axis, depression and suicidality. European Psychiatry, 25(5), 278-280.

Watson, K. K., Li, D., Brent, L. J. N., Horvath, J. E., Gonzalez-Martinez, J., Lambides, A. R., Robinson, A. G., Skene, J. H. P., \& Platt, M. L. (2015). Genetic influences on social attention in free-ranging rhesus macaques. Animal Behaviour, 103, 267-275.

Wedemeyer, C., Goutman, J. D., Avale, M. E., Franchini, L. F., Rubinstein, M., \& Calvo, D. J. (2007). Functional activation by central monoamines of human dopamine D4 receptor polymorphic variants coupled to GIRK channels in Xenopus oocytes. European Journal of Pharmacology, 562(3), 165-173.

Weeland, J., Overbeek, G., de Castro, B. O., \& Matthys, W. (2015). Underlying mechanisms of gene-environment interactions in externalizing behavior: a systematic review and search for theoretical mechanisms. Clinical Child and Family Psychology Review, 18(4), 413-442.

Wendland, J. R., Hampe, M., Newman, T. K., Syagailo, Y., Meyer, J., Schempp, W., Timme, A., Suomi, S. J., \& Lesch, K. P. (2006). Structural variation of the monoamine oxidase A gene promoter repeat polymorphism in nonhuman primates. Genes, Brain and Behavior, 5(1), 40-45.

Wendland, J. R., Lesch, K. P., Newman, T. K., Timme, A., Gachot-Neveu, H., Thierry, B., \& Suomi, S. J. (2006). Differential functional variability of serotonin transporter and monoamine oxidase A genes in macaque species displaying contrasting levels of aggression-related behavior. Behavior Genetics, 36(2), 163-172.

Wersinger, S. R., Ginns, E. I., O'carroll, A. M., Lolait, S. J., \& Young Iii, W. S. (2002). Vasopressin V1b receptor knockout reduces aggressive behavior in male mice. Molecular Psychiatry, 7(9), 975-984.

Wertz, J., Bair, T. B., \& Chimenti, M. S. (2016). PyVar: an extensible framework for variant annotator comparison. BioRxiv, 78386.

Whitehead, H., \& Dufault, S. (1999). Techniques for analyzing vertebrate social structure using identified individuals. Advances in the Study of Behavior, 28, 33-74. 
Wieacker, P., \& Steinhard, J. (2010). The prenatal diagnosis of genetic diseases. Deutsches Ärzteblatt International, 107(48), 857-862.

Williams, K. L., \& Galliher, R. V. (2006). Predicting depression and self-esteem from social connectedness, support, and competence. Journal of Social and Clinical Psychology, 25(8), 855-874.

Williams, T. N. (2006). Human red blood cell polymorphisms and malaria. Current Opinion in Microbiology, 9(4), 388-394.

Willour, V. L., Chen, H., Toolan, J., Belmonte, P., Cutler, D. J., Goes, F. S., Zandi, P. P., Lee, R. S., MacKinnon, D. F., Mondimore, F. M., Schweizer, B., DePaulo Jr., J. R., Gershon, E. S., McMahon, F. J., Potash, J. B., DePaulo, J. R., Gershon, E. S., McMahon, F. J., \& Potash, J. B. (2009). Family-based association of FKBP5 in bipolar disorder. Molecular Psychiatry, 14(3), 261-268.

Wilson, A. J., Gelin, U., Perron, M.-C., \& Réale, D. (2009). Indirect genetic effects and the evolution of aggression in a vertebrate system. Proceedings of the Royal Society of London B: Biological Sciences, 276(1656), 533-541.

Wilson, V. A. D., Humle, A. W. T., \& Udono, N. M. T. (2017). Chimpanzee personality and the arginine vasopressin receptor 1A genotype. Behavior Genetics, 47(2), 215226.

Wilson, A. P., \& Boelkins, R. C. (1970). Evidence for seasonal variation in aggressive behaviour by Macaca mulatta. Animal Behaviour, 18, 719-724.

Winslow, J. T., Noble, P. L., Lyons, C. K., Sterk, S. M., \& Insel, T. R. (2003). Rearing effects on cerebrospinal fluid oxytocin concentration and social buffering in rhesus monkeys. Neuropsychopharmacology, 28(5), 910.

Witte, A. V., \& Flöel, A. (2012). Effects of COMT polymorphisms on brain function and behavior in health and disease. Brain Research Bulletin, 88(5), 418-428.

Wittig, R. M., Crockford, C., Lehmann, J., Whitten, P. L., Seyfarth, R. M., \& Cheney, D. L. (2008). Focused grooming networks and stress alleviation in wild female baboons. Hormones and Behavior, 54(1), 170-177.

Wittig, R. M., Crockford, C., Weltring, A., Deschner, T., \& Zuberbühler, K. (2015). Single aggressive interactions increase urinary glucocorticoid levels in wild male chimpanzees. PLOS ONE, 10(2), 1-22.

Wittig, R. M., Crockford, C., Weltring, A., Langergraber, K. E., Deschner, T., \& Zuberbühler, K. (2016). Social support reduces stress hormone levels in wild chimpanzees across stressful events and everyday affiliations. Nature Communications, 7, 13361.

Wolf, J. B., Brodie III, E. D., Cheverud, J. M., Moore, A. J., \& Wade, M. J. (1998). Evolutionary consequences of indirect genetic effects. Trends in Ecology \& Evolution, 13(2), 64-69.

Worley, G., Page, G., Goldstein, R., \& Cotten, M. (2015). Polygenic risk score of hypothalamic-pituitary-adrenal reactivity and neurodevelopmental outcomes of extremely low birth weight. Developmental Medicine \& Child Neurology, 57(S5), 108. 
Wrangham, R. W. (2018). Two types of aggression in human evolution. Proceedings of the National Academy of Sciences of the United States of America, 115(2), 245-253.

Wray, N. R., Goddard, M. E., \& Visscher, P. M. (2007). Prediction of individual genetic risk to disease from genome-wide association studies. Genome Research, 17(10), $1520-1528$.

Yavich, L., Forsberg, M. M., Karayiorgou, M., Gogos, J. A., \& Männistö, P. T. (2007). Site-specific role of catechol-O-methyltransferase in dopamine overflow within prefrontal cortex and dorsal striatum. Journal of Neuroscience, 27(38), 1019610209.

Yehuda, R. (1997). Sensitization of the hypothalamic-pituitary-adrenal axis in posttraumatic stress disorder. Annals of the New York Academy of Sciences, 821(1), $57-75$.

Young, E. A., Aggen, S. H., Prescott, C. A., \& Kendler, K. S. (2000). Similarity in saliva cortisol measures in monozygotic twins and the influence of past major depression. Biological Psychiatry, 48(1), 70-74.

Young, C., Hähndel, S., Majolo, B., Schülke, O., \& Ostner, J. (2013). Male coalitions and female behaviour affect male mating success independent of dominance rank and female receptive synchrony in wild Barbary macaques. Behavioral Ecology and Sociobiology, 67(10), 1665-1677.

Young, C., Majolo, B., Heistermann, M., Schülke, O., \& Ostner, J. (2014a). Responses to social and environmental stress are attenuated by strong male bonds in wild macaques. Proceedings of the National Academy of Sciences of the United States of America, 111(51), 18195-18200.

Young, C., Majolo, B., Schülke, O., \& Ostner, J. (2014b). Male social bonds and rank predict supporter selection in cooperative aggression in wild Barbary macaques. Animal Behaviour, 95, 23-32.

Yudofsky, S. C., Stevens, L., Silver, J., Barsa, J., \& Williams, D. (1984). Propranolol in the treatment of rage and violent behavior associated with korsakoff's psychosis. American Journal of Psychiatry, 141(1), 114-115.

Yudofsky, S. C., Silver, J. M., Jackson, W., Endicott, J., \& Williams, D. (1986). The overt aggression scale for the objective rating of verbal and physical aggression. The American Journal of Psychiatry, 143(1), 35-39.

Zai, C. C., de Luca, V., Strauss, J., Tong, R. P., Sakinofsky, I., \& Kennedy, J. L. (2012). Genetic factors and suicidal behavior. In Dwivedi, Y. (Ed.), The Neurobiological Basis of Suicide, 213-235. Boca Raton: CRC Press.

Zammit, S., Jones, G., Jones, S. J., Norton, N., Sanders, R. D., Milham, C., McCarthy, G. M., Jones, L. A., Cardno, A. G., \& Gray, M. (2004). Polymorphisms in the MAOA, MAOB, and COMT genes and aggressive behavior in schizophrenia. American Journal of Medical Genetics Part B: Neuropsychiatric Genetics, 128(1), 19-20.

Zhao, J., Jiang, C., Lam, T. H., Liu, B., Cheng, K. K., Xu, L., Au Yeung, S. L., Zhang, W., Leung, G. M., \& Schooling, C. M. (2014). Genetically predicted testosterone and cardiovascular risk factors in men: a mendelian randomization analysis in the guangzhou biobank cohort study. International Journal of Epidemiology, 43(1), 
$140-148$.

Zhou, Z., Zhu, G., Hariri, A. R., Enoch, M.-A., Scott, D., Sinha, R., Virkkunen, M., Mash, D. C., Lipsky, R. H., Hu, X.-Z., Hodgkinson, C. A., Xu, K., Buzas, B., Yuan, Q., Shen, P.-H., Ferrell, R. E., Manuck, S. B., Brown, S. M., Hauger, R. L., Stohler, C. S., Zubieta, J.-K. \& Goldman, D. (2008). Genetic variation in human NPY expression affects stress response and emotion. Nature, 452(7190), 997-1001.

Zhou, Q., Wei, H., Huang, Z., \& Huang, C. (2011). Diet of the Assamese macaque Macaca assamensis in limestone habitats of Nonggang, China. Current Zoology, 57(1), 18-25.

Zhu, M., \& Zhao, S. (2007). Candidate gene identification approach: progress and challenges. International Journal of Biological Sciences, 3(7), 420-427.

Zinner, D., Fickenscher, G. H., \& Roos, C. (2013). Family Cercopithecidae (Old World monkeys). In Mittermeier, R. A., Rylands, A. B., \& Wilson, D. E. (Eds.), Handbook of the Mammals of the World - Volume 3 Primates, 550-627. Barcelona: Lynx Edicions.

Zinner, D., Wertheimer, J., Liedigk, R., Groeneveld, L. F., \& Roos, C. (2013). Baboon phylogeny as inferred from complete mitochondrial genomes. American Journal of Physical Anthropology, 150(1), 133-140.

Zubieta, C., He, X.-Z., Dixon, R. A., \& Noel, J. P. (2001). Structures of two natural product methyltransferases reveal the basis for substrate specificity in plant Omethyltransferases. Nature Structural \& Molecular Biology, 8(3), 271-279.

Zubieta, J.-K., Heitzeg, M. M., Smith, Y. R., Bueller, J. A., Xu, K., Xu, Y., Koeppe, R. A., Stohler, C. S., \& Goldman, D. (2003). COMT val158met genotype affects microopioid neurotransmitter responses to a pain stressor. Science, 299(5610), 1240-1243.

Zuk, O., Hechter, E., Sunyaev, S. R., \& Lander, E. S. (2012). The mystery of missing heritability: genetic interactions create phantom heritability. Proceedings of the National Academy of Sciences of the United States of America, 109(4), 1193-1198.

Zuker, M. (2003). Mfold web server for nucleic acid folding and hybridization prediction. Nucleic Acids Research, 31(13), 3406-3415. 


\section{Curriculum vitae}

\section{Personal information}

Name: $\quad$ Daria Raffaella Gutleb

Date of birth: $21^{\text {st }}$ October 1991

Nationality: Austrian

E-mail: _ daria@gutleb.at

\section{Education}

2015-2018 PhD in Behavior and Cognition, University of Goettingen, Germany

2012-2014 MSc in Zoology, University of Vienna, Austria

2009-2012 BSc in Biology/Zoology, University of Vienna, Austria

\section{Grants}

2015 Research Grant, German Research Foundation

2014 International Communication funding, Austrian Research Association

2010-2013 Performance scholarships, University of Vienna

\section{Journal articles}

in preparation Gutleb DR, Roos C, Heistermann M, Noll A, Ostner J, Schülke O. A multi-locus genetic risk score modulates social buffering of HPA axis activity in wild male primates

2018 Gutleb DR, Roos C, Noll A, Ostner J, Schülke O. COMT Val ${ }^{158}$ Met moderates the link between rank and aggression in a non-human primate, Genes, Brain and Behavior, 17:e12443 

A. Non-invasive genotyping with a massively parallel sequencing panel for the detection of SNPs in HPA-axis genes, Scientific Reports, 8:15944 Pflüger LS, Gutleb DR, Hofer M, Fieder M, Wallner B, Steinborn R. Allelic variation of the COMT gene in a despotic primate society: A haplotype is related to cortisol excretion in Macaca fuscata, Hormones and Behavior 78:220-230

Gutleb DR, Pflüger LS, Wallner B. Semi-freie und wilde Japanmakaken eine Meta-Analyse, Carinthia II 204:433-442 contrast of female faces: Attraction of attention and its dependence on male hormone status in Macaca fuscata, Animal Behaviour 94:61-71

\section{Conference contributions}

2018 Schülke O, Gutleb DR, Heistermann M, Noll A, Roos C, Ostner J. Genetic risk predicts cortisol levels, risk-taking, and social bonding behavior of male Assamese macaques (talk), 13. Jahrestagung der Ethologischen Gesellschaft, Potsdam, Germany

2017 Gutleb DR, Roos C, Heistermann M, Noll A, Schülke O, Ostner J. Genetic load of the hypothalamic-pituitary-adrenal-axis is associated with physiological stress and risk-taking in wild macaques (poster), $11^{\text {th }}$ Göttinger Freilandtage, Göttingen, Germany

2017 Gutleb DR, Roos C, Heistermann M, Noll A, Schülke O, Ostner J. Contribution of the human COMT $\mathrm{Val}^{158} \mathrm{Met}$ polymorphism to aggressive behaviour and stress in a non-human primate (poster), Behaviour Conference, Lisbon, Portugal

Gutleb DR, Heistermann M, Schülke O, Ostner J. Changes in glucocorticoid excretion after intergroup encounters in male Assamese macaques (talk), $15^{\text {th }}$ Conference of the Gesellschaft für Primatologie (GfP), Zurich, Switzerland 

Steinborn R, Wallner B, Pflüger LS. Is conflict behaviour of male Japanese macaques associated with polymorphisms in FKBP5? (poster), $8^{\text {th }}$ European Conference on Behavioural Biology, Vienna, Austria

2015 Gutleb DR, Roos C, Schülke O, Ostner J. Impacts of genotype on stress in wild Assamese macaques (poster), $36^{\text {th }}$ Thai Wildlife Meeting, Bangkok, Thailand

2015 Gutleb DR, Pflüger LS, Hofer M, Fieder M, Wallner B, Steinborn R. Methodological considerations for faecal DNA genotyping on the example of macaques (poster), $14^{\text {th }}$ Conference of the Gesellschaft für Primatologie (GfP), Leipzig, Germany

Pflüger LS, Gutleb DR, Hofer M., Fieder M., Wallner B, Steinborn R. Allelic variation of the COMT gene in a despotic primate society: A haplotype is related to cortisol excretion in Macaca fuscata (poster), $14^{\text {th }}$ Conference of the Gesellschaft für Primatologie (GfP), Leipzig, Germany

2014 Gutleb DR. Demographic variability and steroid excretion of wild and semi-free Japanese macaques: a comparative approach (talk), International Conference on Evolution and Behaviour 2014, Zagreb, Croatia

2014 Gutleb DR, Pflüger LS, Wallner B. Semi-free versus wild Japanese macaques - a meta-analysis of endocrine and demographic parameters (poster), International Conference on Diseases of Zoo and Wild Animals 2014, Warsaw, Poland 